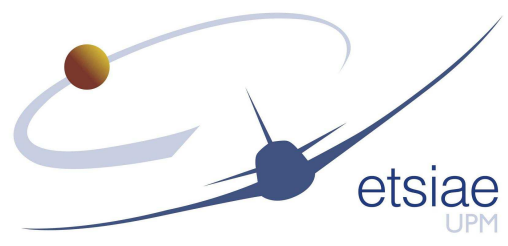

UNIVERSIDAD POLITÉCNICA DE MADRID

ESCUELA TÉCNICA SUPERIOR DE INGENIERÍA AERONÁUTICA Y DEL ESPACIO

\title{
PROPAGATOR INTEGRAL METHOD FOR PLASMA PHYSICS KINETIC EQUATIONS WITH PRACTICAL APPLICATIONS
}

\author{
Tesis Doctoral \\ Jorge González Muñoz \\ Máster en Ingeniería Aeroespacial
}





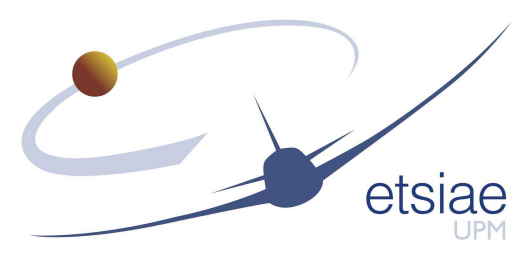

DEPARTAMENTO DE FÍSICA APLICADA A LA INGENIERÍA AERONÁUTICA Y NAVAL ESCUELA TÉCNICA SUPERIOR DE INGENIERÍA AERONÁUTICA Y DEL ESPACIO

PROPAGATOR INTEGRAL METHOD FOR PLASMA PHYSICS KINETIC EQUATIONS WITH PRACTICAL APPLICATIONS

\author{
Tesis Doctoral
}

\author{
Jorge González Muñoz \\ Máster en Ingeniería Aeroespacial \\ Director: Jose Manuel Donoso Vargas \\ Doctor en Ciencias Físicas
}





\section{POLITÉCNICA}

El tribunal nombrado por el Sr. Rector Magnífico de la Universidad Politécnica de Madrid, el día de de $20 \ldots$.

Presidente:

Vocal:

Vocal:

Vocal:

Secretario:

Suplente:

Suplente:

Realizado el Acto de Defensa y Lectura de la Tesis el día de de 20....... en la E.T.S.I./Facultad

Calificación

EL PRESIDENTE

LOS VOCALES

EL SECRETARIO 

A mis padres.

A mi hermana.

A Yaizza.

A mi abuela. 



\section{AGRADECIMIENTOS}

Esta tesis ha sido posible gracias al apoyo y ayuda de muchísimas personas durante casi cuatro años. Espero ser capaz de agradeceros a todos lo que vuestro apoyo ha supuesto.

En primer lugar, quiero agradecer a los profesores Luis Conde, Juan Luis Domenech, Ezequiel del Río y, en especial, a mi director Jose Manuel Donoso. Gracias por aceptarme desde el primer día, buscando unas cualidades que pocas personas buscan en nuestro entorno. Me habéis transmitido una gran pasión por la ciencia y, sobre todo, por la enseñanza. Gracias por formarme no sólo como una persona científica, si no también para ser una científica persona. Habéis sido unos maestros excelentes, no podría pedir mejores guías.

Sandra, gracias por abrir camino. Ha sido un reto seguir tus huellas, y sin ti este proceso habría sido muchísimo más duro complicado. Gracias por todo lo que me has enseñado, científica y no científicamente hablando.

Quiero agradecer también a Domenico Giordano por todo lo que me ha enseñado, expandiendo mis conocimientos en áreas que para mí sonaban extrañas hace unos años y que me ha abierto la puerta a trabajar en multitud de campos y centros de investigación de alto nivel en Europa.

I would like to thank to all the people in the DLR in Göttingen for the time I spent there, and specially to Volker Hannemann for the fruitful discussions we had about many topics in physics and engineering.

Quiero agradecer a todos los compañeros con los que he compartido despacho. I would like to thank all my office partners: Fer, Julius, Pablo y Tobias. Muchas gracias por los buenos y malos momentos que hemos compartido. Thank you for the good and bad times we have shared.

También quiero agradecer a todos los miembros del departamento de Física Aplicada 
a las Ingenierías Naval y Aeronáutica por su colaboración, en especial a M. ${ }^{\mathrm{a}}$ Carmen Velasco Gómez por siempre atender con gran rapidez todas mis peticiones. También he de agradecer al Personal de Administración y Servicios y de Cafetería por su ayuda y apoyo, en especial a María García Guijarro por toda su ayuda, especialmente con los trámites burocráticos iniciales en los que estaba extremadamente perdido.

Gracias a toda la gente del TS por los buenos ratos y las risas compartidas, habéis sido un constante apoyo estos años y espero que sigáis muchos años más en mi vida. También quiero dar las gracias a los hostiles, que no se creían que esta tesis fuese verdad y era una excusa para no quedar con ellos, os debo unos cachopos.

Quiero agradecer especialmente a Yaizza por su apoyo, por sacarme de casa cuando más lo necesitaba y por aguantarme en los malos momentos estos años, especialmente en el final de esta tesis. Te mereces todo lo mejor del mundo, y yo como tu amigo voy a estar siempre ahí para apoyarte e intentar dártelo. Muchas gracias también por llenar mi casa de alegría junto a Ari, Dani y Nacho.

Y por último y más importante, quiero agradecer a mis padres y a mi hermana. Sin vuestro apoyo esta tesis no habría podido ni empezar a gestionarse. Gracias por animarme siempre, por esperar lo mejor de mí y por no dejar de apoyarme nunca. Si estoy aquí es gracias a vosotros. También quiero recordar a mi abuela, a quien le habría encantado ver la persona en la que me he convertido gracias a todos vosotros.

Finally, I would like also to thank all the members of this evaluation committee, for their willingness, dedication, interest and commentaries.

A todos vosotros, muchas gracias.

Veinticuatro horas al día, siete días por semana.

No hay encargo lo bastante difícil, ni factura lo bastante alta.

Peter Venkman

To all of you, thank you very much.

Twenty-four hours a day, seven days a week.

No job is too big, no fee is too big.

Peter Venkman 


\section{ABSTRACT}

Plasmas are of great interest for aerospace engineering applications, such as surface treatment for metals and composites or to electric propulsion, among others. Improvements in plasma knowledge affects these and many other fields related to science and engineering, as for example measurement processes by electric probes or plasma-wall interaction. Numerical analyses are nowadays a fundamental tool to obtain approximated solutions of plasma model equations, in both fluid and kinetic approaches. However, available commercial software packages usually miss the required tools to physically describe these particular phenomena. Thence, the development of specific numerical methods, able to provide physical descriptions of these scenarios, is an important task involving many fields of mathematics, physics and engineering. In aerospace applications, Weakly Ionized Plasmas (WIP) are very common in astrophysical environments and in laboratory. These plasmas are characterised by low temperatures and low charged densities as well as non-thermal situations. In this work, the Propagator Integral Method (PIM) is proposed as an alternative to classical numerical schemes, to solve problems in the field of Kinetic Theory for plasmas, with a special focus on WIP. This method is founded in advancing the solution problem by means of transition probability density functions, which are short-time approximations effectively working as Green's functions. Therefore, the method works as a semi-analytical procedure, since emerges form stochastic theory and numerical integration being brought together, resulting in a stable method, not based upon finite differences. Propagators, and in general path integral methods, have been widely applied to solve drift-diffusive equations, also dealt in this work. Additionally, here the PIM is extended to address purely convective and ordinary differential equations, which commonly appear in kinetic descriptions of plasmas. Here the PIM is proved to be highly suitable to cover non-linear problems involving discontinuities or sharp distribution functions evolutions that may naturally arise. A wide variety of cases is solved to illustrate the capability of the PIM to compute cumbersome equations, finding evolutions not available with other numerical schemes. Engaged situations, as the self-consistent resolution of a 
three-species plasma or the plasma-wall interaction, are solved and new models of interest, to characterising the WIP generated in the UPM PlasmaLab, are proposed. In these models, each species evolves under disparate time and spatial scales but they are advanced with the same time step. The purely kinetic simulations allow the computation of fluxes that are qualitatively compared with experimental data. This opens a new view of the analysis of transport processes, of capital importance in practical applications and in connection to fluid dynamics. The description of the ion population generated by an electric thruster is also studied. The results directly impact the development of plasma thrusters and experimental measurements. Besides, the ideas and models proposed here can be easily translated to other plasma types, since pure kinetic resolutions allows the inclusion of a wide range of microscopic processes. 


\section{RESUMEN}

Las aplicaciones de plasmas, como por ejemplo el tratamiento de superficies en metales y materiales compuestos o la propulsión eléctrica, son de gran importancia para la ingeniería aeroespacial. El avance en el conocimiento del plasma afecta a estos y otros campos relacionados con la ciencia y la ingeniería, como procesos de medición y la interacción plasma-pared. Los análisis numéricos son hoy en día una herramienta fundamental para obtener soluciones aproximadas de las ecuaciones en modelos de plasma, tanto fluidos como cinéticos. Sin embargo, el software comercial disponible no cuenta con las herramientas necesarias para describir físicamente estos fenómenos. Como consecuencia, el desarrollo de métodos numéricos específicos capaces de proporcionar descripciones físicas de estos escenarios es una tarea importante que involucra varios campos de la matemática, la física y la ingeniería. En aplicaciones aeroespaciales, los Plasma Débilmente Ionizados (WIP) son muy comunes en entornos astrofísicos y en el laboratorio. Estos plasmas se caracterizan por una baja temperatura y densidad de carga, así como de no estar en equilibrio térmico. En este trabajo, el Método Integral de Propagadores (PIM) es propuesto, como alternativa a esquemas numéricos clásicos, para resolver los problemas en el campo de la Teoría Cinética de plasmas, con especial atención a WIP. Este método está basado en avanzar la solución mediante funciones de probabilidad de transición, que son aproximaciones efectivas a tiempos cortos de funciones de Green específicas. Por lo tanto, el método funciona como semi-analítico, ya que emerge de la combinación de la teoría estocástica y la integración numérica, resultando en un esquema estable no basado en diferencias finitas. Propagadores, y en general métodos de integral de camino, han sido ampliamente usados para resolver ecuaciones de advección-difusión, también tratadas en este trabajo. Adicionalmente, el PIM es extendido para tratar con ecuaciones puramente advectivas y ecuaciones diferenciales ordinarias, comunes en descripciones cinéticas de plasmas. Aquí, el PIM demuestra ser adecuado para resolver problemas no lineales que incluyen discontinuidades o evoluciones abruptas de la función de distribución. Una amplia variedad de casos son resueltos para demostrar la capacidad del PIM para resolver ecuaciones intrincadas, encontrando 
evoluciones no disponibles con otros esquemas numéricos. Situaciones escabrosas, como la resolución auto-consistente de un plasma de tres especies o la interacción plasma pared, se resuelven y nuevos modelos, de interés para la caracterización del WIP generado en el laboratorio del UPM PlasmaLab, se proponen. En estos modelos, cada especie evoluciona en escalas temporales y espaciales dispares, pero son avanzadas con el mismo paso temporal. Las simulaciones puramente cinéticas permiten el cálculo de flujos que son cualitativamente comparados con datos experimentales. Esto abre una nueva visión el cálculo de procesos de transporte, de gran importancia en aplicaciones prácticas, y relacionados con la dinámica de fluidos. La descripción de iones generada por un motor eléctrico es también estudiada. Los resultados afectan directamente el desarrollo de motores de plasma y medidas experimentales. Además, las ideas y modelos planteados aquí pueden ser fácilmente trasladados a otros tipos de plasma, dado que la resolución puramente cinética permite incluir una amplia variedad de procesos microscópicos. 


\section{CONTENTS}

Abstract

Resumen $\quad$ v

1 A brief introduction to plasmas and computation 1

1.1 Numerical methods in Plasma Physics . . . . . . . . . . . . . . 2

1.2 Thesis scope . . . . . . . . . . . . . . . . 3

2 Propagator Integral Method 5

2.1 Applications to general partial differential equations . . . . . . . . . 6

2.2 Fokker-Planck equation . . . . . . . . . . . . . . 8

2.2.1 Scheme for one-dimensional problems . . . . . . . . . . 10

2.2 .2 Extension to $1+1 \mathrm{D}$ problems . . . . . . . . . . . 15

2.2 .3 Extension to $2 \mathrm{D}$ problems $\ldots \ldots \ldots \ldots \ldots$

2.2 .4 Numerical hindrances . . . . . . . . . . . . . . . . . . . . 19

2.2.5 Comparison with FDM and computational cost . . . . . . . . 21

2.3 Purely convective equations . . . . . . . . . . . . . . . . . 24

2.3.1 The non-diffusive one-dimensional problem . . . . . . . . 25

2.3.2 Extension to 2D problems . . . . . . . . . . . . . . . 29

2.3 .3 Numerical hindrances . . . . . . . . . . . . . . . . . . . . . 32

2.3.4 Comparison with FDM and computational cost . . . . . . . . 34

2.4 Ordinary Differential Equations . . . . . . . . . . . . . . 36

2.4.1 One-dimensional Poisson's equation . . . . . . . . . 37

2.4 .2 Numerical hindrances . . . . . . . . . . . . . . . . . . . . . 39

2.4.3 Comparison with FDM and computational cost . . . . . . . . 40

2.5 Conclusions . . . . . . . . . . . . . . . . . . . 41

3 An introduction to Plasma Physics $\quad 43$

3.1 Kinetic theory in plasmas . . . . . . . . . . . . . . . . 44

3.2 Fluid theory . . . . . . . . . . . . . . . . . . . 47

3.3 Collisionless plasma $\ldots \ldots \ldots \ldots \ldots$ 
3.3.1 Evolution of one species affected by a constant force . . . . . . 50

3.3.2 Two charged species with self-consistent electric field . . . . . . 52

3.4 Conclusions . . . . . . . . . . . . . . . . . . . . 58

4 Description of particle collisions $\quad 59$

4.1 Elastic collisions . . . . . . . . . . . . . . . . . 60

4.1.1 The Fokker-Planck-Landau (FPL) term . . . . . . . . . . . 61

4.1.2 Hard Spheres potential case . . . . . . . . . . . . . . . . . 64

4.1.3 Dougherty collision term . . . . . . . . . . . . . 65

4.1.4 One-dimensional Dougherty term . . . . . . . . . . . . . 67

4.1.5 The 1D charge-charge term . . . . . . . . . . . 70

4.1 .6 BGK . . . . . . . . . . . . . . . . . . . . 74

4.1 .7 Entropy evolution considerations . . . . . . . . . . . . 78

4.2 Influence of self-collisions in shocks . . . . . . . . . . . . . 79

4.3 Charge-exchange . . . . . . . . . . . . . . . . . . 82

4.4 Conclusions . . . . . . . . . . . . . . . . . . 85

$\begin{array}{llr}5 & \text { Weakly Ionized Plasmas } & \mathbf{8 7}\end{array}$

5.1 One dimensional three species kinetic model . . . . . . . . . . . . . . 89

5.1.1 Influence of collisions in the dynamics of WIP . . . . . . . . . 93

$5.1 .2 \quad$ Abrupt electric field . . . . . . . . . . . . . . . . . 96

5.1.3 Temperature dependent collision frequencies . . . . . . . . . . 98

5.1 .4 Ionization ratio . . . . . . . . . . . . . . . . . . . 100

5.2 Analysis of discrepancies in WIP characterisation . . . . . . . . . . 107

5.2.1 A short review of the experimental setup and results . . . . . . 108

5.2 .2 Numerical results . . . . . . . . . . . . . . . . . . . . . . . . 112

5.3 Transport coefficients for electrons in WIP . . . . . . . . . . . 119

5.4 Conclusions . . . . . . . . . . . . . . . . . . . . . . . 121

6 Plasma-wall interaction $\quad \mathbf{1 2 3}$

6.1 Emissive wall as a discontinuous plasma medium . . . . . . . . . . . . . 124

6.1 .1 Influence of background pressure . . . . . . . . . . . 127

6.1 .2 Influence of the the wall potential . . . . . . . . . . . 134

6.1.3 Realistic collisional scale without electric field . . . . . . . . . . 137

6.2 Collecting wall . . . . . . . . . . . . . . . . . . 138

6.2 .1 Effect of ions dynamics . . . . . . . . . . . . . . . . 142

6.2 .2 Influence of charge-neutral collisions . . . . . . . . . . . . 144

6.3 Conclusions . . . . . . . . . . . . . . . . . . . . . . . . . . . . 144

$\begin{array}{lll}7 & \text { Characterisation of a plasma plume } & 147\end{array}$

7.1 Experimental motivation . . . . . . . . . . . . . . . . . . 149

7.2 Ion kinetic description in the thruster plume . . . . . . . . . . . . . . 149

7.2.1 Influence of charge-exchange and elastic collisions . . . . . . . . 151

7.2 .2 Effect of the initial ion velocity . . . . . . . . . . . . 154

7.3 Conclusions . . . . . . . . . . . . . . . . . . 156 
8 Conclusions $r$

8.1 Future lines of research . . . . . . . . . . . . . . . . . . 161

A Collisions in PIC with PIM $\quad 165$

A.1 The weighted random scattering based on the distribution function . . . 166

A.2 A practical example: a 1D1V Brownian motion . . . . . . . . . . . 169

A.3 A non-linear example, the Kappa 1D1V motion . . . . . . . . . . . . . 171

A.4 Conclusions . . . . . . . . . . . . . . . . . . . . 172

B Numerical listings and subroutines $\quad \mathbf{1 7 5}$

B.1 Computation time reduction with OpenMP . . . . . . . . . . 175

B.2 Rectangle integration method . . . . . . . . . . . . . . . . 177

B.3 Diffusion correction for conservation of energy . . . . . . . . . . 178

B.4 Adaptable mesh . . . . . . . . . . . . . . . . . . . . . 179

B.5 Interpolation of a non-constant mesh . . . . . . . . . . . . . . . . 181

B.6 Simple vs. double precision variables . . . . . . . . . . . . . . 183

B.7 Convergence criteria for plasma-wall discontinuous model . . . . . . . . 185

$\begin{array}{lr}\text { C List of publications and conferences } & 187\end{array}$ 



\section{LIST OF FIGURES}

2.1 Evolution of the one-dimensional kappa problem and its integral quantities . . . . . . . . . . . . . . . . . 12

2.2 Evolution of the discontinuous drift-diffusion problem . . . . . . . . . 13

2.3 Evolution of the bounded kappa problem . . . . . . . . . . . . . 14

2.4 Evolution of the bounded absorption problem . . . . . . . . . . . 15

2.5 Evolution of $f(x, v, t)$, its norm and fluxes $\ldots \ldots \ldots \ldots$

2.6 Zones where boundary conditions for the Klein-Kramers problem can be imposed . . . . . . . . . . . . . . . . . 17

2.7 Evolution of $f=F / 2 \pi v_{\perp} \ldots \ldots \ldots \ldots \ldots$

2.8 Evolution of the macroscopic quantities in both directions . . . . . . . 19

2.9 Evolution of the function $f(q, t)$ for different values of $D \ldots \ldots$

2.10 Solution $f(x, v, 0.5)$ for two values of the time advancing parameter . . . 21

2.11 Evolution of an histogram-like initial condition solved by the PIM, central and upwind finite difference schemes . . . . . . . . . . . . 22

2.12 Same problem as in figure 2.11 but with $\Delta q=0.04 \ldots \ldots \ldots 22$

2.13 A situation where $q_{i^{\prime}+1}$ does not match $q_{i^{\prime}}+D_{i^{\prime}} \tau \ldots \ldots \ldots . \ldots . \ldots 25$

2.14 Resolution of a simple convective problem with a fixed mesh and the one recalculated by equation $(2.52) \ldots \ldots \ldots \ldots$

2.15 Norm of the pure convective case with fixed and adaptable meshes . . . 26

2.16 Solution of the Burgers equation for different values of $D_{q q} \ldots \ldots . .27$

2.17 Evolution of $f(q, t)$ for numerical and analytical solutions $\ldots \ldots . .29$

2.18 Grid displacement in a $1 \mathrm{D} 1 \mathrm{~V} x-v$ space for $F=0 \ldots \ldots . \ldots . . \ldots 30$

2.19 Evolution of $f(x, v x t)$ and its interpolation for $F=-\operatorname{sgn}(x) \ldots \ldots 31$

2.20 First three order moments of the interpolated function . . . . . . . . 31

2.21 Evolution of $f(x, v x t)$ and its interpolation for $F=-x \ldots \ldots 32$

2.22 First three order moments of the interpolated function . . . . . . . . . . 32

2.23 Initial and final mesh for non-homogeneous convective 1D problem . . . 33

2.24 Evolution of an histogram-like initial condition solved by the PIM, central and upwind finite difference schemes . . . . . . . . . . . . . 34 
2.25 Percentage of the iteration time employed by each task of a 1D1V purely convective simulation . . . . . . . . . . . . . . 35

2.26 Solution for Poisson's equation with a source-sink term equals $q$, a diffusion of the form $1+q^{2}$ and Dirichlet boundaries . . . . . . . . . . 38

2.27 Solution for the Poisson's equation in the semi-open domain . . . . . . 39

2.28 Resolution of Poisson's equation with a non-homogeneous term and a diffusion coefficient equal $q$ and $1+q^{2}$ respectively . . . . . . . . . 40

3.1 Classification of plasmas by electron density and temperature . . . . . 43

3.2 Evolution of the distribution function in the phase-space . . . . . . . . 51

3.3 Flux of particles with and without the external histogram-like force . . . 51

3.4 Particle density with and without the external histogram-like force . . . 52

3.5 Evolution of electrons under a self-consistent electric field between a positive potential jump . . . . . . . . . . . . . . . . . 53

3.6 Evolution of ions under a self-consistent electric field between a positive potential jump ....................... . . 53

3.7 Fluxes for electrons and ions on the left axis and electric field on the right axis ....................... . . 54

3.8 Evolution of electrons a under self-consistent electric field with no potential difference at the boundaries . . . . . . . . . . . . . 55

3.9 Evolution of ions under a self-consistent electric field with no potential difference at the boundaries . . . . . . . . . . . . . . . 55

3.10 Electron and ion maximum distribution point trajectory . . . . . . . 56

3.11 Evolution of fluxes under a self-consistent electric field with no potential difference at the boundaries . . . . . . . . . . . . . . . 57

3.12 Evolution of electron flux and self consistent electric field with fixed and

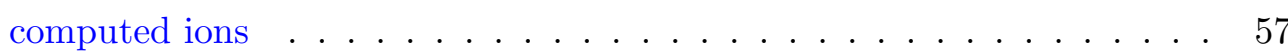

4.1 Distribution function $f\left(v_{\perp}, v_{\|}, t\right)$ for the case of self-collisional FPL term . . . . . . . . . . . . . . . . . . .

4.2 Balance of macroscopic variables and errors respect to the initial condition for the FPL collisional term . . . . . . . . . . . . . . .

4.3 Distribution function $f\left(v_{\perp}, v_{\|}, t\right)$ for the case of self-collisional HS term . . . . . . . . . . . . . . . . . .

4.4 Balance of macroscopic variables and errors respect to the initial condition for the HS collisional term . . . . . . . . . . . . . . . 65

4.5 Distribution function $f\left(v_{\perp}, v_{\|}, t\right)$ for the case of self-collisions with a $2 \mathrm{~V}$ Dougherty term . . . . . . . . . . . . . . 66

4.6 Balance of macroscopic variables and errors respect to the initial condition for the $2 \mathrm{~V}$ Dougherty collisional term . . . . . . . . . . . . 66

4.7 Evolution of the self-collisional Dougherty 1D operator . . . . . . . . 68

4.8 Numerical errors of the conserved quantities respect to initial condition for the one-dimensional Dougherty collision term . . . . . . . . . . . 
4.9 Evolution of the conserved properties for the two-species collisional Dougherty operator . . . . . . . . . . . . . . .

4.10 Evolution of the distribution function for the two-species collisional Dougherty operator . . . . . . . . . . . . . . . 69

4.11 Evolution of the self-collisional one-dimensional charge-charge operator

4.12 Numerical errors of the conserved quantities respect to the initial condition for the one-dimensional charge-charge collision term . . . . . . 72

4.13 Evolution of the conserved properties for the two-species one-dimensional charge-charge collision operator . . . . . . . . . . . . 73

4.14 Evolution of the distribution function for the two-species collisional onedimensional charge-charge operator . . . . . . . . . . . . 73

4.15 Evolution of the self-collisional BGK operator . . . . . . . . . . . 75

4.16 Numerical errors of the conserved quantities respect to initial condition for BGK collision term . . . . . . . . . . . . . . . 75

4.17 Evolution of the distribution function for two different collision terms relaxing into a Maxwellian of

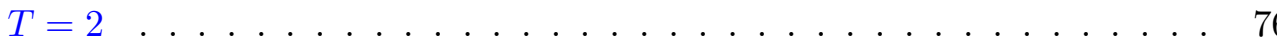

4.18 Evolution of the macroscopic moments for two different collision terms and its difference . . . . . . . . . . . . . . . 76

4.19 Evolution of the conserved properties for the two-species collisional BGK

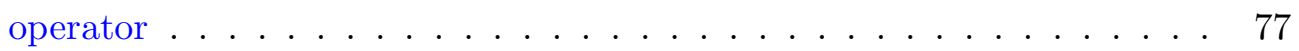

4.20 Evolution of the distribution function for the two-species collisional BGK operator . . . . . . . . . . . . . . . . 77

4.21 Boltzmann entropy evolution for the self-collision terms studied . . . . . 79

4.22 Velocity evolution after the discontinuous initial condition . . . . . . . 80

4.23 Distribution function at $t=0.25$ for the non-collisional and the two collisional cases . . . . . . . . . . . . . . . . . 81

4.24 Macroscopic velocity for both species for different constants values

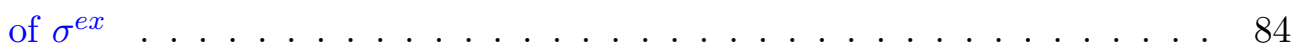

4.25 Temperature for both species for different constants values of $\sigma^{e x} \ldots .84$

4.26 Distribution function for both species at $t=0.1 \ldots \ldots 85$

5.1 Representation of the charge and neutral densities respect to the total one as a function of the ionization ratio . . . . . . . . . 88

5.2 Representation of amounts of neutrals, ions and electrons . . . . . . . 93

5.3 Evolution of mass fluxes for electrons, ions and neutrals . . . . . . . . 94

5.4 Distribution function at $t=1 \mathrm{~ms}$ is presented for electrons, ions and neutrals ............................. 95

5.5 Evolution of mass fluxes for electrons, ions and neutrals . . . . . . . 96

5.6 Mass fluxes for electrons, ions and neutrals . . . . . . . . . . . . 97

5.7 Zoomed view of figure $5.6 \mathrm{a} \ldots \ldots \ldots$. . . . . . . . . . . . 97

5.8 Evolution of species temperatures for different values of $E_{0} \ldots \ldots 98$

5.9 Comparison of the electron mass flux with variable and constant collision parameters . . . . . . . . . . . . . . . . . 99 
5.10 Comparison of the ions mass flux with variable and constant collision parameters . . . . . . . . . . . . . . . . . . 99

5.11 Mass fluxes for the three species for $r_{i}=10^{-6}, 10^{-5}, 3 \cdot 10^{-5}$ and $10^{-4} .101$

5.12 Zoomed view of figure 5.11a when electric field changes . . . . . . . . 101

5.13 Flux before the change in electric field as a function of the ionization ratio . . . . . . . . . . . . . . . . . . . 102

5.14 Temperature of the three species for $r_{i}=10^{-6}, 10^{-5}, 3 \cdot 10^{-5}, 7 \cdot 10^{-5}$

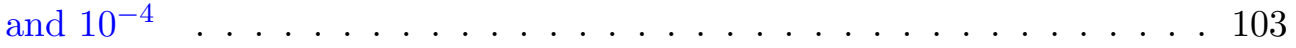

5.15 Steady fluxes versus the ionization ratio for $E_{0}=0.3 \mathrm{~V} / \mathrm{m}$. . . . . . . . . 104

5.16 Temperature of the three species for $r_{i}=10^{-5}, 10^{-4}, 3 \cdot 10^{-4}, 7 \cdot 10^{-4}$

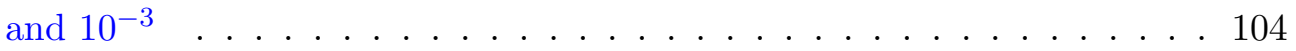

5.17 Steady mass fluxes for $n_{T}=2 \cdot 10^{13} \mathrm{~cm}^{-13} \ldots \ldots \ldots \ldots$. . . . . . 105

5.18 Temperatures for the three species with a total density of $2 \cdot 10^{13} \mathrm{~cm}^{-13} \quad 105$

5.19 Steady mass fluxes for $n_{T}=2 \cdot 10^{13} \mathrm{~cm}^{-13}$ and $E_{0}=0.3 \mathrm{~V} / \mathrm{m} \ldots \ldots 106$

5.20 Fluxes at the end of the linear zone for the different cases studied in this section . . . . . . . . . . . . . . . . 106

5.21 Steady fluxes with and without charge-charge collisions . . . . . . . . 107

5.22 Langmuir probe and electric circuit employed to bias it . . . . . . . 109

5.23 Voltage shape of the signals applied to the Langmuir probe and theoretical representation of an I-V curve . . . . . . . . . . . . . . . . 110

5.24 Experimental $\mathrm{I}-\mathrm{V}$ curves of a collecting probe for signals at $f_{s w}=20$, 55,108 and $249 \mathrm{~Hz} \ldots \ldots \ldots \ldots \ldots$. . . . . . . . . . . . . . . . . . . .

5.25 Time evolution of $J_{e}$ for a non-collisional plasma, a collisional plasma without neutrals and the three species one-dimensional model . . . . . . 112

$5.26 \mathrm{~J}_{e}$-E curves obtained with temperature dependent collision frequencies . 114

5.27 Zoomed view of the results in figure 5.26 corresponding to the starting point of the different measurement edges . . . . . . . . . . . . 115

$5.28 \mathrm{~J}_{e}$-E curves obtained with constant collision frequencies $\ldots \ldots \ldots$

5.29 Zoomed view of the results in figure 5.28 corresponding to the starting point of the different measurement edges . . . . . . . . . . . . . 117

$5.30 \mathrm{~J}_{e}$-E curves obtained with constant collision frequencies $\ldots \ldots \ldots$

5.31 Electron temperature, density current and heat flux in the parallel direction . . . . . . . . . . . . . . . . . . 120

6.1 Emitted current at $x=0$ and $x=0.02 \mathrm{~mm} \ldots \ldots \ldots \ldots 127$

6.2 Experimental measures of the current emitted by a wire . . . . . . . 128

6.3 Contours of $f_{e} \times 10^{-4}$ at $T_{w}=2000 \mathrm{~K} \ldots \ldots \ldots \ldots$. . . . . . . . . . . . 129

6.4 Contours of $f_{e} \times 10^{-2}$ at $T_{w}=2300 \mathrm{~K} \ldots \ldots \ldots \ldots \ldots \ldots \ldots$

6.5 Electric field close to the wall for high temperatures . . . . . . . . 130

6.6 Electric potential $\phi$ for different temperatures and pressures studied. . . 131

6.7 Comparison between the emitted current with and without the selfconsistent electric field at $x=0 \mathrm{~mm} \ldots \ldots \ldots \ldots \ldots$

6.8 Comparison between the emitted current with and without the selfconsistent electric field at $x=0.02 \mathrm{~mm} \ldots \ldots \ldots \ldots$ 
6.9 Emitted current $J_{T}$ at $x=0.02 \mathrm{~mm}$ for different wall potentials $\phi_{w} \ldots 134$

6.10 Electric potential for $\phi_{w}=-3 \mathrm{~V} \ldots \ldots \ldots \ldots \ldots \ldots$

6.11 Saturation potential and current as a function of wall temperature and pressure . . . . . . . . . . . . . . . 136

6.12 Emitted current as a function of temperature and background pressure for realistic collisional scales . . . . . . . . . . . . . . . . . . 137

6.13 Density current for electrons and ions . . . . . . . . . . . . . . 140

6.14 Contours of $f_{e} \times 10^{-7}$ at different time frames . . . . . . . . . . . 141

6.15 Electric field and potential close to a collecting wall . . . . . . . . . 142

6.16 Comparison between electron evolution with and without advancing the ions in time . . . . . . . . . . . . . . . . . . . 143

6.17 Comparison between self-consistent electric field with and without advancing the ions in time . . . . . . . . . . . . . . . 143

6.18 Density current at different values of cross-section . . . . . . . . . . 144

7.1 ALPHIE thruster and its scheme . . . . . . . . . . . . . . . 148

7.2 Measurements of the ion energy distribution function by means of a RPA . . . . . . . . . . . . . . . . . . . . . 149

7.3 Influence of the charge-exchange in the ion distribution function . . . 152

7.4 Influence of the charge-exchange in the current density and temperature . . . . . . . . . . . . . . . . . . 152

7.5 Influence of the elastic collision in the ion distribution function . . . . . 153

7.6 Influence of the elastic collision in the current density and temperature . 153

7.7 Distribution function for different values of $K_{0} \ldots \ldots \ldots$. . . . . . 154

7.8 Evolution of accelerated and thermal ion distribution function maxima . 155

7.9 Evolution of the ion current density and temperature as a function of $K_{0} \ldots \ldots \ldots \ldots \ldots \ldots \ldots \ldots$

7.10 Ion current density as a function of $K_{0} \ldots \ldots \ldots \ldots$

A.1 Weighted distribution of random particles as a function of $N_{\text {total }}$

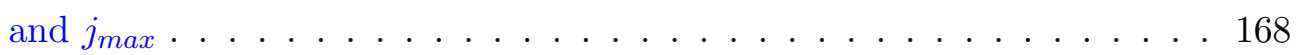

A.2 Evolution of particles in the $x-v$ domain for different scenarios . . . . . 170

A.3 Time evolution of density for the three solved problems . . . . . . . 171

A.4 Evolution of two different drift-diffusive effects: a $\kappa$ and Brownian motions . . . . . . . . . . . . . . . . . 172

B.1 Computational time as a function of the number of threads . . . . . 177

B.2 Effect of the different corrections . . . . . . . . . . . . . 179

B.3 Interpolation of numerical solution for problem presented in figure 2.19 . 183

B.4 Interpolation of numerical solution for problem presented in figure 2.19 but with a double Gaussian initial function . . . . . . . . . . . . 183

B.5 Errors of the macroscopic quantities for the self-collisional one-dimensional Dougherty operator with double and simple precision . . . . . . . . . . 184

B.6 Burgers' inviscid problem in double and simple precision . . . . . . . . 184

B.7 Required number of iterations to reach convergence . . . . . . . . . . . 185 



\section{LIST OF TABLES}

2.1 Specifications of the computer employed to compare computational times among numerical methods . . . . . . . . . . . . . 23

2.2 Computational times per iteration and total time for each method employed to solve (2.43) with $\Delta q=0.004$. . . . . . . . . . . . . . . . . 23

2.3 Computational times per iteration and total time for each method employed to solve equation (2.66) with $\Delta q=0.004$. . . . . . . . . . 35

2.4 Boundary conditions for ODE problem . . . . . . . . . . . . . . . 37

2.5 Computational times for the PIM and direct matrix inversion method (Gauss) to solve Poisson's equation . . . . . . . . . . . . . 41

4.1 Mass, density, velocity and temperature for the two species studied in the binary collision . . . . . . . . . . . . . . . . 61

4.2 Initial macroscopic values that generate a time evolving shock . . . . . . 80

5.1 Mass, temperature and density employed to represent an argon WIP . . 92

5.2 Argon plasma to compute transport coefficients . . . . . . . . . . . 119

6.1 Argon plasma to analyse the collecting wall . . . . . . . . . . . . . . 139

6.2 Electron plasma frequencies close to the collecting wall . . . . . . . . . 140

7.1 Initial conditions for neutrals and ions . . . . . . . . . . . . . . 150 



\section{LISTINGS}

B.1 Reduction of time calculation for the propagator with OpenMP . . . 176

B.2 Simple subroutine to compute the integral of $f \ldots \ldots \ldots$

B.3 Generation of the new grid . . . . . . . . . . . . 180

B.4 Interpolation subroutine . . . . . . . . . . . . . . . . . . 181 



\section{CHAPTER 1}

\section{A BRIEF INTRODUCTION TO PLASMA PHYSICS AND NUMERICAL COMPUTATION}

The first definition of a plasma appears before 1930 in [1] and [2] by I. Langmuir and L. Tonks to describe the electronic and ionic oscillations in an ionized gas. Since then, the interest in plasmas has greatly grown, as has also happened with the knowledge of the phenomena that appear in these systems. However, accurate descriptions of plasmas either from theoretical or experimental points of view, are still of major importance to fully understand and extend the applicability of these peculiar systems.

A wide-range of applications has been developed for plasmas over the past century such as light sources [3], energy generation in fusion devices [4], surface treatment of materials $[5,6]$, water purification [7] or electric propulsion [8] are some of the main applications of plasmas. Also, plasmas appear naturally in the universe. Independently of its application or origin, plasmas always present a collective response to perturbations in the electromagnetic field. Nevertheless, due to the wide-range in which plasmas can exist, specific descriptions are required to account (or neglect) some phenomena depending on the case of interest. This leads to situations where, for example, a model employed to analyse a laser inducted plasma cannot be applied to describe the plume of an ion thruster. This obstructs the development of new models and complicates the inter-applicability and exchange of knowledge among the different branches of Plasma Physics. However, the similarity between some microscopic phenomena provides a common ground between different areas of Plasma Physics. The individual study of these phenomena from a kinetic point of view is of major importance, and results obtained can be applied to develop new models for different plasma types. This is a necessity to improve the knowledge of current plasma applications and develop new ones that push the frontier of science and technology. 


\subsection{Numerical methods in Plasma Physics}

Due to its high complexity, plasma descriptions usually require numerical resolutions in order to obtain functional results, either for steady state or in time-evolving situations. Over the years, a large variety of methods and schemes have been developed to deal with the equations that arise from the description of multi-species plasmas. Among the multiple methods, some of them highlight due to its applicability, as the Navier-Stokes solvers for magnetohydrodynamics (MHD) [9,10], Euler-Vlasov (EV) solvers [11], and particle-in-cell (PIC) codes [12-17]. Specially, PIC codes have gained a large popularity over the years and have proven an outstanding tool to analyse the dynamics of a large variety of plasmas. These methods compute the kinematic motion of a large number of particles, that represent the different species in the plasma, due to acceleration produced by electromagnetic forces and calculate the particle density and other macroscopic variables in a fixed mesh by means of a weighted interpolation.

However, actual numerical methods have some difficulties to introduce some physical processes as collisions or to deal with the different time scales that appear in plasmas due to disparate rates in density, mass or temperature among the different species. Nevertheless, some progress has been performed to address this, e.g., to introduce collisions into PIC codes through Monte Carlo Collision method [14,17]; providing good descriptions of the microscopic interaction among particles, as elastic collisions or ionization-recombination processes. Nevertheless, it is an arduous task to implement particle interactions in PIC or other codes as they are convoluted non-linear processes. This results in these effects being ignored even when they may become relevant [18], leading to the utilization of simplified models, to accommodate the limitations of actual numerical tools, which can provide inaccurate results or non-physical behaviours and may fail to explain relevant processes found in plasmas.

The search of numerical methods that improve the actual state of the art and provide new results, accounting for more troublesome phenomena, must always be pursued. This will allow, for example, to properly describe situations where collisions have a relevant influence in plasma dynamics $[19,20]$. In this line, new approaches to solve involved non-linear coupled systems that allow a more accurate description of plasmas must be always stimulated $[18,21]$.

This dissertation proposes the use of a semi-analytical method founded on transition probability functions to solve the kinetic equations that arise from the different descriptions of plasmas and providing the complete distribution function as a solution. The goal is to obtain physical meaningful results, accounting for all possible microscopic phenomena, that can lead to expand the knowledge of plasmas in general, and improve the characterization of plasmas by experimental means and the development of electric thrusters in particular, as these two topics are of major importance for our research group. 


\subsection{Thesis scope}

This thesis is grounded on the previous Master Final Project [21]. In that work, the semi-analytical Propagator Integral Method (PIM) [22-27] was used to solve a two species one-dimensional plasma (electrons and ions) close to a wall described by a drift-diffusion fluid model and accounting for the self-consistent electric field through the Poisson's equation. The main goal of this thesis is to apply the PIM to solve equations that commonly appear in the study of plasmas from a Kinetic Theory perspective, including convection-diffusion, purely convective problems or the Poisson's equation. The method is applied to solve arduous models, accounting for a variety of physical effects of disparate intensity, that are very difficult to implement with classical numerical methods, specially in Weakly Ionized plasmas (WIP), were collisions play a very relevant role $[25,28]$. Similar methods, based on propagators or other integral kernels, have been employed to solve a wide range of problems in physics and engineering [29-33]. For example, descriptions of climate models [34], cells [35] or blood vessels [36] can be analysed using methods founded on the same principles as the PIM.

This dissertation is divided as follows.

Chapter 2 is devoted to review the semi-analytical Propagator Integral Method to provide a basic knowledge of its foundation and present the diverse applications developed in the recent years. The procedure to solve convective-diffusion equations is reviewed and studied in deep, extending the application presented in previous works, as $[23,26,27]$. Additionally, the method is extended for the first time to deal with new differential problems as pure convective or ordinary equations. Boundary conditions are added to allow the resolution of confined problems, reviewing the implementation presented by J. M, Donoso in [27]. Also, relevant numerical results for each equation type are presented to illustrate some test-cases, useful to understand how this method works. These examples represent simplified cases of the more complex simulations performed in the following chapters. In addition, the typical numerical hindrances found during the development and application of the method are presented for each particular equation kind, as well as simple comparison with classical finite difference methods. It is relevant to analyse these topics in here as they are strongly related to the numerical implementation of the method and the physical evolutions obtained.

Chapter 3 contains a brief introduction to Plasma Physics and specially to Kinetic Theory, setting the grounds to understand the nomenclature employed through out this thesis. In addition, some simulations are computed to analyse the evolution of non-collisional plasmas under a self-consistent electric field. These examples are useful to understand the dynamics in the phase-space composed by position and velocity, very common in kinetic problems and to check the validity of the PIM to study this type of plasmas never addressed before with this method.

Chapter 4 comprises a study of the different collision terms employed in this work and 
other particle interaction phenomena, as charge-exchange. Conservation properties for self and binary collisions are analytically and numerically studied. These analyses are relevant to identify dynamics caused by collisional processes that appear in further chapters, specially when species of disparate energy, density and mass ratios interact. Besides, the validity of the PIM to explore conservative problems is stressed out as it is important for the semi-analytical method to fulfil the analytical macroscopic evolutions.

Chapter 5 focus on the analysis of Weakly Ionized Plasmas (WIP) from a kinetic point of view. To achieve this task, a one-dimensional three species kinetic model is introduced and numerically studied under a variety of scenarios, such as abrupt time evolving electric field or different ionization ratios. Then, this model is employed to analyse a discrepancy found when a Langmuir probe is biased with different sweep signals to characterize a WIP. In addition, transport coefficients for these plasmas are computed for a range of electric field and ionization ratio. These calculations are performed in a velocity space defined by the directions parallel and perpendicular to the electric field to better represent the collisional dynamics. Moreover, the complete distribution function is computed in these cases as no linearisations are required, which allows a representation of a wide range of situations far from equilibrium.

Chapter 6 presents the models proposed in this dissertation to deal with the emission and collection of charged particles by a metallic wall from a kinetic point of view. This is a very common situation in plasmas, specially in laboratory applications where experiments are usually bounded by metallic walls. The results found are qualitatively similar to experimental data and provide an useful first approach to understand how electrons and ions behave close to a metallic wall.

In chapter 7 , the modelisation of the plume produced by an electric thruster is carried out. The main process that produce the distribution of ions measured in the laboratory by means of a Retarding Potential Analyser (RPA) are examined. These numerical simulations are useful to better understand the characterization of these systems and the mechanisms that affect the ions produced by the thruster.

Finally, in chapter 8, a summary of the main results presented in this dissertation is introduced as conclusions, as well as the future lines of research in which this work can serve as a foundation for new works.

Three appendixes are added after the conclusions. Simple guidelines about the possibility of employing short-time propagators to incorporate collisions in PIC codes are presented in appendix A. In appendix B, some numerical recipes employed in the codes developed for this thesis are presented for the sake of clarity. Finally, appendix C contains the lists of publications, conferences, seminars, and projects delivered as a consequence of this work. 


\section{CHAPTER 2}

\section{PROPAGATOR INTEGRAL METHOD}

Numerical methods suitable to deal with complex non-linear problems providing, at the same time, stable and physical meaningful time-evolving solutions are of huge importance in many fields of mathematics, physics and engineering. Numerical solutions are always approximated to the analytical ones, usually unknown, and they are not free of errors beyond the approximation order. However, these methods allow the resolution of challenging problems that are unsolvable otherwise. Thence, the search of new procedures able to reduce or control these errors and deal with more involved problems, keeping a stable physical solution, must be continuously stimulated.

Under this perspective, integral methods $[22,29,31-33,37-40]$ provide a robust semianalytical scheme to obtain a suitable time evolving solution for a great variety of problems. The lack of discretisation that characterises these schemes allows to obtain consistent transient and steady solutions even if abrupt or sharp parameters, initial or boundary conditions appear. Through out this dissertation, a semi-analytical integral method has been developed and structured to solve convoluted equations that usually appear in the kinetic description of plasmas. However, the procedures presented here can be extended to other fields without loosing any of the advantages and capabilities of the integral scheme, a feature that turns it in a method of general use in fields where similar equation types appear.

In this context, this chapter is devoted to study the advances and applications of the Propagator Integral Method, PIM for short, developed in the recent years. Basic testcases are solved here to illustrate and state the methodology, paying special attention to equations similar to those appearing in the plasma kinetic descriptions. In addition, a systematic procedure to apply this method to any type of equation is operatively presented. As it is shown in chapter 3, these equations are, but not restricted to drift- 
diffusion, purely convective and the Poisson's equation. The first type of equations has been widely dealt in the past by our research group [21-26, 41-44] and by other authors $[29,31-33,39,40]$. Here, a general procedure to obtain short-time solutions as propagators, including also the possible boundary effects, is revisited and expanded. Then, the method is applied, for the first time, to purely convective partial differential equations, as well to ordinary differential equations. This chapter is useful to establish some common ground to understand the simulations of the following parts in this dissertation, where the PIM is used to solve involved models. The numerical implementation and comparison with classical discretisation methods are included, since they are of major importance to remark some of the PIM advantages.

\subsection{Applications to general partial differential equations}

To start with the mentioned PIM extension, let us assume a general partial differential equation in the form of a governing equation for a problem of stochastic nature as

$$
\begin{aligned}
\mathfrak{L}(\mathbf{q}, t)[f(\mathbf{q}, t)] & =s(f, \mathbf{q}, t), \\
f(\mathbf{q}, 0) & =f_{0}(\mathbf{q})
\end{aligned}
$$

where $\mathbf{q}$ is any set of coordinates that represents the problem in a domain $\mathfrak{D}, \mathfrak{L}(\mathbf{q}, t)$ is a general differential operator, $s(f, \mathbf{q}, t)$ is a non-homogeneous term, $t$ represents time, $f(\mathbf{q}, t)$ is the problem function at any time $t>0$ and $f_{0}(\mathbf{q})$ is the initial condition required to have a complete Cauchy problem. Equation (2.1) normally includes boundary effects symbolised as

$$
\Gamma_{\partial \mathfrak{D}}[f(\mathbf{q}, t)]=g(f, \mathbf{q}, t)
$$

where $\Gamma_{\partial \mathfrak{D}}$ is any integro-differential operator that acts at the domain edge $\partial \mathfrak{D}$. In many instances, these problems can be solved, analytically, by using general methods based on integral kernels resembling the form of the solution, in terms of Green's functions [45-50], as

$$
f(\mathbf{q}, t)=\int_{\mathfrak{D}} G\left(\mathbf{q}, t ; \mathbf{q}^{\prime}, 0\right) f_{0}\left(\mathbf{q}^{\prime}\right) \mathrm{d} \mathbf{q}^{\prime}+\int_{0}^{t} \int_{\mathfrak{D}} G\left(\mathbf{q}, t ; \mathbf{q}^{\prime}, T\right) s\left(\mathbf{q}^{\prime}, T\right) \mathrm{d} \mathbf{q}^{\prime} \mathrm{d} T,
$$

where $G\left(\mathbf{q}, t ; \mathbf{q}^{\prime}, 0\right)$ fulfils equation (2.1) without non-homogeneous term and with the initial condition $G\left(\mathbf{q}, t ; \mathbf{q}^{\prime}, t\right)=\delta\left(\mathbf{q}-\mathbf{q}^{\prime}\right)$ and the same boundary conditions as the original problem. This function represents how the value of $f_{0}$ evolves from a source point $\mathbf{q}^{\prime}$ into a field point $\mathbf{q}$ in a time $t$, first integral of (2.3) right hand side, and the effect of the non-homogeneous term, second integral of (2.3) right hand side.

If an integral kernel is employed, a similar approach can be derived. Assuming that a transition operator $U_{t, t^{\prime}}$ exists in the form

$$
f(\mathbf{q}, t)=U_{t, t^{\prime}} f\left(\mathbf{q}^{\prime}, t^{\prime}\right)=\int_{\mathfrak{D}} f\left(\mathbf{q}^{\prime}, t^{\prime}\right) \Pi\left(\mathbf{q}, t ; \mathbf{q}^{\prime}, t^{\prime}\right) \mathrm{d} \mathbf{q}^{\prime}
$$


and it is valid for a time $t>t^{\prime}$ where non-homogeneous terms have been neglected as they are introduced as an external effect, independently of the transition probability. The integral kernel $\Pi\left(\mathbf{q}, t ; \mathbf{q}^{\prime}, t^{\prime}\right)$ is usually called the propagator associated to the transition operator. This operator fulfils the initial condition $U_{t, t}=1$ or, equivalently, $\Pi\left(\mathbf{q}, t ; \mathbf{q}^{\prime}, t\right)=\delta\left(\mathbf{q}-\mathbf{q}^{\prime}\right)$. The integral kernel can be employed in equation (2.3) by substituting the Green's function and modifying the time integral limits to evolve from $t^{\prime}$ to $t$ instead of from 0 to $t$. Thence, $\Pi$, can be understood as a representation on how a solution at $\mathbf{q}^{\prime}$ time $t^{\prime}$ evolves for $t>t^{\prime}$ to the point represented by field coordinates $\mathbf{q}$ at time $t$. However, either the Green's function or the integral kernel $\Pi$ are, usually, as difficult to obtain as the complete solution of the problem.

Nevertheless, a short-time approximation for $\Pi$ can be usually found, called short-time propagator, see [22] and references therein. This function provides an approximated time evolution when it is employed in numerical integral schemes. Usually, this shorttime propagator can be computed analytically for a vast number of cases, but other approaches may include the numerical calculation of this function, as presented in [51].

To introduce the general procedure that allows the calculation of short-time propagators, firstly we assume a solution of the problem to solve at an instant $t^{\prime}$ and position $\mathbf{q}^{\prime}$ exits. Due to the operator $\mathfrak{L}(\mathbf{q}, t), f\left(\mathbf{q}^{\prime}, t^{\prime}\right)$ in the source state, represented by primes, propagates to the field points $\mathbf{q}$ at a time $t>t^{\prime}+\tau$, where $\tau$ is a small parameter. If we call $\Pi\left(\mathbf{q}, t ; \mathbf{q}^{\prime}, t^{\prime}\right)$ to the probability of $f\left(\mathbf{q}^{\prime}, t^{\prime}\right)$ to reach position $\mathbf{q}$ at time $t$, an approximation of $\Pi$ can be found solving the auxiliary problem

$$
\frac{\partial P_{\tau}}{\partial \tau}=\mathfrak{L}^{*}\left(\mathbf{q}^{\prime}, t^{\prime}\right)\left[P_{\tau}\right]
$$

where $\mathfrak{L}^{*}\left(\mathbf{q}^{\prime}, t^{\prime}\right)$ is the same operator as in $(2.1)$ but evaluated at source variables $[22$, $23,45,49,52]$ and $P_{\tau}=P_{\tau}\left(\mathbf{q} ; \mathbf{q}^{\prime}\right) \simeq \Pi\left(\mathbf{q}, t ; \mathbf{q}^{\prime}, t-\tau\right)$ is the short-time approximation of the real unknown transition probability. The resolution of this problem is usually simple since parameters involved in it are formally constant. Nevertheless, geometrical factors or bounded domains may result in very difficult auxiliary equations, demanding an arduous analytical analysis to be solved. Thence, this method is considered as semianalytical as it is founded in analytical integral scheme and calculation of propagators, but requires a numerical integrations to advance the solution.

If the propagator is solved as in equation (2.5), no control over the boundary conditions is possible, apart of to the natural ones of the propagator. To include boundary effects, the condition of the general problem (2.1) must be included in the propagator calculation as $\Gamma_{\partial \mathfrak{D}}\left[P_{\tau}\right]=0$, as seen in [27] for the one-dimensional Fokker-Planck equation in the semi-open domain. Nevertheless, other approaches to account for boundary conditions can be used, as indicated in the reference cited above. A large number of $P_{\tau}$ candidates can be found in the textbook [49] by K. D. Cole stated for heat equations. Thence, imposing boundary conditions in integral schemes is not a trivial task $[50,52-55]$. In this chapter, some general procedure to include boundary effects is presented, but the general problem is far from being solved. Nevertheless, any method 
to include non-homogeneous boundary conditions should add an additional term to the advancing scheme, that includes the effect of the boundary to the advancing scheme as

$$
\begin{aligned}
f\left(\mathbf{q}, t^{\prime}+\tau\right)= & \int_{D} P_{\tau}\left(\mathbf{q} ; \mathbf{q}^{\prime}\right) f\left(\mathbf{q}^{\prime}, t^{\prime}\right) \mathrm{d} \mathbf{q}^{\prime}+ \\
& \int_{t^{\prime}}^{t^{\prime}+\tau} \int_{\mathfrak{D}} P_{\tau-\tau^{\prime}}\left(\mathbf{q} ; \mathbf{q}^{\prime}\right) s\left(\mathbf{q}^{\prime}, t^{\prime}+\tau^{\prime}\right) \mathrm{d} \mathbf{q}^{\prime} \mathrm{d} \tau^{\prime}+f_{B},
\end{aligned}
$$

where $f(\mathbf{q}, t+\tau)$ denotes the approximation to the real solution obtained by means of the short time approximation, $\tau^{\prime}$ represents an intermediate time between $t$ and $t+\tau, P_{\tau-\tau^{\prime}}$ accounts for the possible dependency of the propagator in short times and $f_{B}$ is the boundary term $[23,52]$. This term depends on the propagator and the method chosen to incorporate boundary conditions. In a general way, $f_{B}$ involves a time integral, as the one that incorporates the non-homogeneous effects and a closed integration over the domain of some function related to the short time propagator.

Depending on the complexity of the non-homogeneous terms, the integration in time and space may become impossible to realise even numerically without simplifying assumptions. For instance, if the integral limits can be interchanged, the contribution of the non-homogeneous term to the advancing scheme (2.21) can be rewritten as

$$
\int_{-\infty}^{\infty} s\left(\mathbf{q}^{\prime}, t+\theta \tau^{\prime}\right) Q_{\tau}\left(\mathbf{q} ; \mathbf{q}^{\prime}\right) \mathrm{d} \mathbf{q}^{\prime}
$$

for some real value such that $0 \leq \theta \leq 1$, being the function $Q_{\tau}$ the integral over time of the propagator as

$$
Q_{\tau}=\int_{0}^{\tau} \mathrm{d} \tau^{\prime} P_{\tau-\tau^{\prime}}\left(\mathbf{q} ; \mathbf{q}^{\prime}\right)
$$

which has the lower and upper limits $\tau \delta\left(\mathbf{q}-\mathbf{q}^{\prime}\right)$ and $\tau P_{\tau}$, respectively, as approximated functions [27]. The final scheme results

$$
f(\mathbf{q}, t+\tau)=\int_{-\infty}^{\infty} P_{\tau}\left(\mathbf{q} ; \mathbf{q}^{\prime}\right) f\left(\mathbf{q}^{\prime}, t\right) \mathrm{d} \mathbf{q}^{\prime}+\int_{-\infty}^{\infty} s\left(\mathbf{q}^{\prime}, t+\theta \tau^{\prime}\right) Q_{\tau}\left(\mathbf{q} ; \mathbf{q}^{\prime}\right) \mathrm{d} \mathbf{q}^{\prime}+f_{B}
$$

At this point, it is important to remark that the propagator form is not unique. Multiple functions can be used as short-time approximations to solve the same problem reaching very similar results. However, the advancing scheme presented here holds as it is independently of the propagator shape.

\subsection{Fokker-Planck (FP) equation}

A Fokker-Planck equation is a partial differential equation which solution evolves by two distinguishable effects: convection and diffusion. Convection, governed by a deterministic formal force, pushes the solution through a privileged direction; whereas 
diffusion, understood as derived from the effect of stochastic nature, spreads the value of one point to, theoretically, all the domain. These equations are very common in mathematics, physics and engineering [47,56-60], and may represent the dynamics of fluids or the evolution of temperature in a body, among many other cases. They are expressed in general shape as

$$
\frac{\partial f}{\partial t}=-\left\{\sum_{i}^{N} \frac{\partial}{\partial q_{i}} D_{i} f-\sum_{i}^{N} \sum_{j}^{N} \frac{\partial^{2}}{\partial q_{i} \partial q_{j}} D_{i j} f\right\}+s=-\mathfrak{L}_{F P} f+s,
$$

where $f=f(\mathbf{q}, t)$ is the problem function, $\mathbf{q}=q_{1}, q_{2} \ldots q_{i} \ldots q_{N}$ is any set of coordinates, $N$ is the dimension number, $D_{i}=D_{i}(f, \mathbf{q}, t)$ is the convective parameter in the direction $i, D_{i j}=D_{i j}(f, \mathbf{q}, t)$ are the components of the symmetric diffusion tensor $\mathbb{D}$ in directions $i$ and $j$, and $s$ is the non-homogeneous term. Here, $\mathfrak{L}_{F P}$ refers to a general operator in the FP form.

To obtain a generic propagator, let us assume we have an open space defined problem with natural boundary conditions, which means that the function and its derivatives respect to $\mathbf{q}$ vanish for large values of $|\mathbf{q}|$. The auxiliary equation that the short-time propagator $P_{\tau}$ must fulfil reads

$$
\frac{\partial P_{\tau}}{\partial \tau}=-\mathfrak{L}_{F P}^{*}\left[P_{\tau}\right]=-\left\{\sum_{i}^{N} D_{i}^{\prime} \frac{\partial}{\partial q_{i}} P_{\tau}-\sum_{i}^{N} \sum_{j}^{N} D_{i j}^{\prime} \frac{\partial^{2}}{\partial q_{i} q_{j}} P_{\tau}\right\},
$$

with the initial condition

$$
\lim _{\tau \rightarrow 0} P_{\tau}\left(\mathbf{q}, \mathbf{q}^{\prime}\right)=\delta\left(\mathbf{q}-\mathbf{q}^{\prime}\right),
$$

where' over the drift and diffusion coefficients indicates that they are evaluated in the source variables, i.e., $D_{i}^{\prime}=D_{i}\left(\mathbf{q}^{\prime}, t^{\prime}\right)$ and $D_{i j}^{\prime}=D_{i j}\left(\mathbf{q}^{\prime}, t^{\prime}\right)$. The simplest shape that solves problem (2.12) is a multi-variable Gaussian distribution as

$$
P_{\tau}\left(\mathbf{q}, \mathbf{q}^{\prime}\right)=\frac{1}{\sqrt{\left|4 \pi \mathbb{D}^{\prime} \tau\right|}} \exp \left[-\frac{\mathbf{Q}^{\top} \cdot \mathbb{D}^{\prime-1} \cdot \mathbf{Q}}{4 \tau}\right] .
$$

Here, $\mathbf{Q}=\mathbf{q}-\mathbf{q}^{\prime}-\mathbf{D}^{\prime} \tau$ is a column vector, $\mathbf{Q}^{\top}$ its transpose and || represents the determinant. This propagator expresses how the $f$ value at source point $\mathbf{q}^{\prime}$ propagates to all field points $\mathbf{q}$ in a short time $\tau$ due to convective and diffusive effects. Commonly used approaches to finite difference methods are unable to reproduce this behaviour, because only the grid points that appear in the discretisation scheme around $\mathbf{q}^{\prime}$ are modified. In this sense, the propagator behaves like a discretisation scheme of order equal to all the points in the mesh or as an implicit finite different method after matrix inversion.

In the analysis of partial differential equations, it is of major importance to compute the evolution of integral quantities. Right now, these quantities are useful to study the dynamics of the numerical problem, but the physical meaning of them is provided in 
chapter 3, where the distribution function moments and fluxes are delineated. These functions are defined as

$$
\begin{aligned}
{\left[q_{i}^{m}\right] } & =\int q_{i} q_{i}^{m} f(\mathbf{q}, t) \mathrm{d} \mathbf{q} \\
\left\langle q_{i}^{n}\right\rangle & =\frac{\int q_{i}^{n} f(q, t) \mathrm{d} \mathbf{q}}{\int f(\mathbf{q}, t) \mathrm{d} \mathbf{q}}
\end{aligned}
$$

where $m=0$ and $1, n=1$ and 2 are usually employed. We define $\langle 1\rangle=\int f(q, t) \mathrm{d} q$ for simplicity, usually referred as the norm. It is easy to see that (2.15) and (2.14) fulfil the relation

$$
\left[q^{m}\right]=\langle 1\rangle\left\langle q^{m+1}\right\rangle
$$

In drift-diffusive equations, these quantities obey the time evolving relations

$$
\begin{aligned}
& \langle\dot{1}\rangle=-\int_{\delta \mathfrak{D}} \mathbf{n} \cdot\left(\mathbf{D} f-\frac{\partial}{\partial \mathbf{q}} \mathbb{D} f\right) \mathrm{d} \mathbf{q}^{\prime}+\int_{\mathfrak{D}} s \mathrm{~d} \mathbf{q} \\
& {\left[\dot{1_{i}}\right]=\int_{\mathfrak{D}} D_{i} f \mathrm{~d} \mathbf{q}-\int_{\delta \mathfrak{D}} q_{i} \mathbf{n} \cdot\left(\mathbf{D} f-\frac{\partial}{\partial \mathbf{q}} \mathbb{D} f\right) \mathrm{d} \mathbf{q}^{\prime}+\int_{\mathfrak{D}} q_{i} s \mathrm{~d} \mathbf{q}}
\end{aligned}
$$

where dotted variables means time derivative and $\mathbf{n}$ is the outwards unit vector normal to the boundary surface. It can be seen that the norm $\langle 1\rangle$ only changes due to boundary or non-homogeneous effects. Other values of $m$ and $n$ follow similar evolution laws, computed by integrating both sides of equation (2.10).

In the following sections, propagators to solve the Fokker-Planck equation in specific geometries and boundary conditions are presented, by significant benchmark problems for each case.

\subsubsection{Scheme for one-dimensional problems}

The general Fokker-Planck equation (2.10) in a one-dimensional open space becomes

$$
\begin{aligned}
\frac{\partial f}{\partial t} & =-\left\{\frac{\partial}{\partial q} D_{q}(q, t) f-\frac{\partial^{2}}{\partial q^{2}} D_{q q}(q, t) f\right\}+s, \\
f(q, 0) & =f_{0}(q)
\end{aligned}
$$

where natural boundary conditions have been assumed for $|q| \rightarrow \infty$. Here, $q$ is the spatial coordinate and the vector $\mathbf{D}$ and stress tensor $\mathbb{D}$ turn into scalars. A propagator that solves this problem can be extracted from (2.13), giving

$$
P_{\tau}\left(q, q^{\prime}, \tau\right)=\frac{1}{\sqrt{4 \pi D_{q q}^{\prime} \tau}} \exp \left[-\frac{\left(q-q^{\prime}-D_{q}^{\prime} \tau\right)^{2}}{4 \tau D_{q q}^{\prime}}\right],
$$

from which equation (2.9) gives rise to the iterative scheme

$$
f(q, t+\tau)=\int_{-\infty}^{\infty} P_{\tau}\left(q ; q^{\prime}\right) f\left(q^{\prime}, t\right) \mathrm{d} q^{\prime}+\int_{-\infty}^{\infty} Q_{\tau}\left(q ; q^{\prime}\right) s\left(q^{\prime}, t+\theta \tau^{\prime}\right) \mathrm{d} q^{\prime} .
$$


This scheme must be translated into the selected numerical domain to allow its resolution, with the Gaussian propagator expressed as

$$
P_{\tau_{i i^{\prime}}}=\frac{1}{\sqrt{4 \pi D_{i^{\prime} i^{\prime}} \tau}} \exp \left[-\frac{\left(q_{i}-q_{i^{\prime}}-D_{i^{\prime}} \tau\right)^{2}}{4 \tau D_{i^{\prime} i^{\prime}}}\right] .
$$

where subscripts $i$ and $i^{\prime}$ are referred to points in the numerical grid. The time advancing scheme can be solved with a simple rectangular integration method as

$$
f_{i}^{n+1}=\sum_{i^{\prime}=0}^{i_{\max }} P_{\tau_{i i^{\prime}}} f_{i^{\prime}}^{n} \Delta q+\sum_{i^{\prime}=0}^{i_{\max }} s_{i^{\prime}}^{n} Q_{\tau_{i i^{\prime}}} \Delta q,
$$

where $n$ represents the actual time step, $i_{\max }$ is the maximum number of points in the grid and $\Delta q$ is the grid distance between two consecutive points. Parameter $\theta$ from equation (2.21) has been set to 0 . Due to obvious limitations in the numerical problem, an infinite grid cannot be employed. In order to correctly approximate the numerical $f$, the grid edges must be far enough to ensure that no relevant value of the solution reaches them. This procedure has proved to be highly feasible in many problems $[22,25]$.

To test this simple advancing scheme, a non-linear problem where convection and diffusion parameters are given by

$$
D_{q}(q, t)=-\gamma q ; D_{q q}(q, t)=\gamma\left(1+\frac{q^{2}}{2 \kappa}\right)
$$

is dealt, being $\gamma$ and $\kappa$ are positive constants. These terms results in a $\kappa$-like steady solution. To ensure that the function $f(q, t)$ has integrable quantities up to order $2, \kappa$ must be greater than $\frac{1}{2}$. When $\kappa \rightarrow \infty$, a steady Gaussian solution is recovered. We take $\kappa=\frac{5}{3}$ and $\gamma=1$. An impulsive non-homogeneous term is also added

$$
s(q, t)=\left\{\begin{array}{ll}
\delta(q-1) & \text { if } 0<t \leq 1 \\
0 & \text { if } 1<t
\end{array} .\right.
$$

where $\delta(x)$ is the Dirac delta. Starting from a zero initial condition $(\langle 1\rangle=0)$, this non-homogeneous term injects information at $q=1$ at a constant rate until the norm of the solution reaches one at $t=1$. So, for $t>1$, the problem evolves freely until a $\kappa$-like steady state is reached.

From a numerical point of view, a grid of 10001 points and a length of 500 nondimensional units centred in $q=0$ is used, with $\tau=10^{-2}$. The large length of the mesh is required due to the power behaviour of the $f$ tails. If not enough length is provided, non small $f$ values may exist at the ends of the numerical domain, perturbing the solution. For the Dirac delta function, a simple numerical representation as

$$
\delta_{n}\left(q_{i}\right)=\left\{\begin{array}{ll}
\frac{1}{\Delta q} & \text { if } q_{i}=0 \\
0 & \text { elsewhere }
\end{array} .\right.
$$




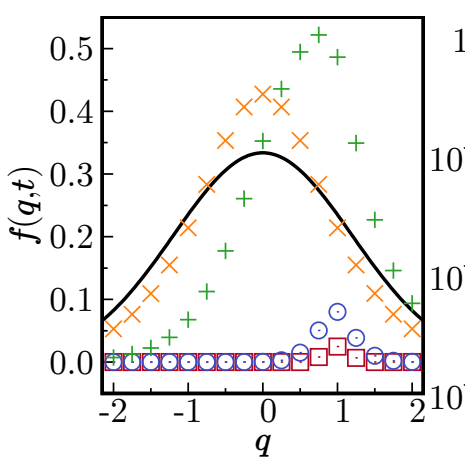

(a) Distribution function

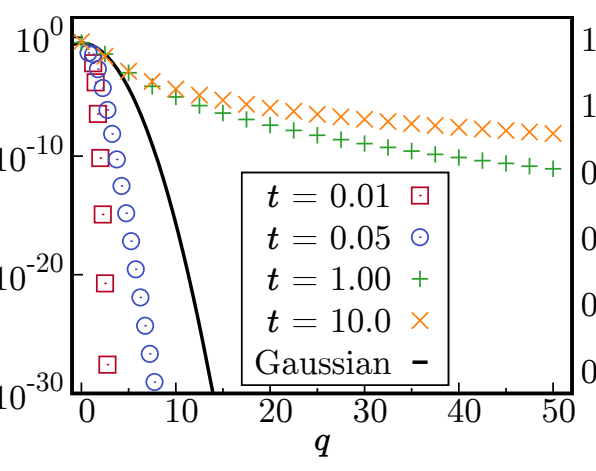

(b) Tails of $f(q, t)$.

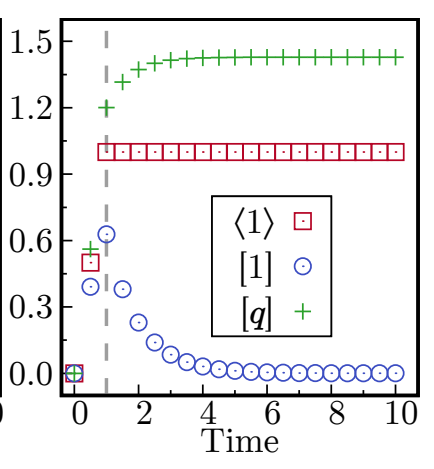

(c) Moments

Figure 2.1: Evolution of the one-dimensional kappa problem and its integral quantities. Black solid line represents the Gaussian function with the same moments that the steady state.

is used keeping a numerical norm equal 1 if a simple rectangle method is used to integrate. Moreover, this representation only acts on one channel of the numerical grid, as it happens with the analytical Dirac delta where only one point in space has a value different from 0 . In addition, in the limit $\Delta q \rightarrow 0$, the analytical Dirac delta is recovered.

From the results shown in figure 2.1, it can be seen how the problem evolves from the transient initial interval, where the delta non-homogeneous term is active, without introducing instabilities in the distribution function or in its moments. From figures 2.1a and $2.1 \mathrm{~b}$ it is clearly appreciated how the quasi-steady solution, at $t=10$, does not match a Gaussian distribution with the same mean and deviation values, pictured as a black solid line. In the $\kappa$-like distribution, $f$ close to $q=0$ differs from an exponential distribution and the solution tails do not decay exponentially, as they follow a power-law as $|v|^{-\kappa+1}$. This is an important result because the Gaussian propagator from equation (2.20) is able to reproduce a non-Gaussian time evolving and steady solutions, an interesting statement useful to solve non-linear problems in kinetic theory where $f$ may deviate from Maxwellian distributions.

If the integral quantities of $f$ are studied, figure 2.1c, smooths evolutions are found, even in the time period in which the Dirac delta source term acts. In addition, it is easy to see how norm $\langle 1\rangle$ follows equation (2.17), as it only changes when the nonhomogeneous term is different from zero and is conserved it afterwards.

Another important application of this integral method developed in the course of this dissertation is the resolution of equations with discontinuous drift-diffusion coefficients [26]. It is based upon the fact that, from a theoretical point of view about Green's functions, it is only required for $P_{\tau}$ to be piecewise continuous. This is useful, for example, to analyse situations where a plasma changes abruptly in time or space, as studied in chapter 6 of this work, or to deal with forces of discontinuous nature. A simple test-case illustrates this issue, where the parameters associated to the 


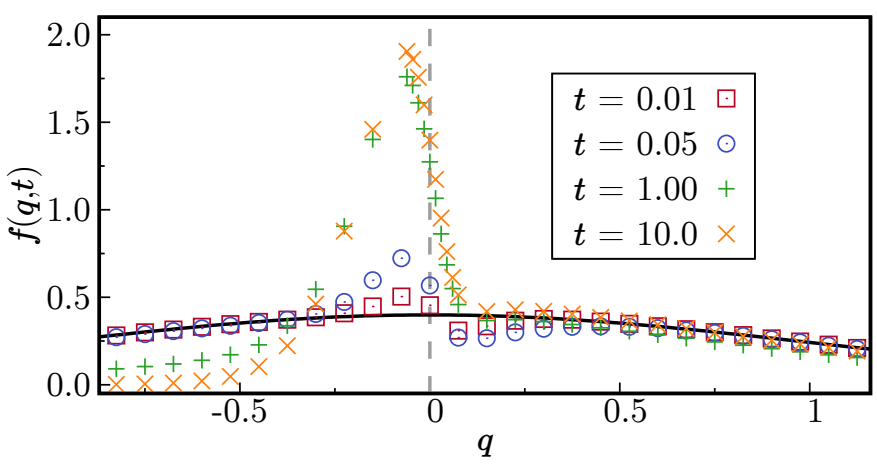

(a) Solution $f(q, t)$ of the problem.

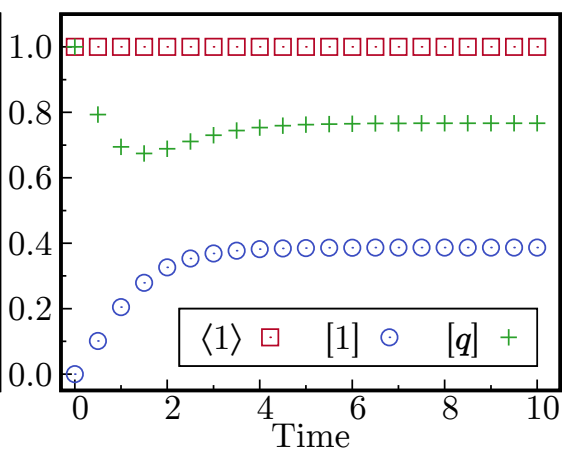

(b) Time evolution of moments

Figure 2.2: Evolution of the discontinuous drift-diffusion problem. Initial condition is presented as a black solid line.

one-dimensional FP equation become

$$
D_{q}(q, t)=\left\{\begin{array}{ll}
1 & \text { if } q \leq 0 \\
-q(1-f) & \text { if } 0>q
\end{array}, D_{q q}(q, t)=\left\{\begin{array}{ll}
0.1 & \text { if } q \leq 0 \\
1+\frac{q^{2}}{2 \kappa} & \text { if } 0>q
\end{array},\right.\right.
$$

the non-homogeneous term $s$ equals 0 and $\kappa=5 / 3$, resulting in a non-linear problem that has the convective term depends on $f$. The solution of this problem has different behaviours depending on the coordinate $q$. For $q<0$, a Gaussian-like solution is expected meanwhile for $q>0$, a $\kappa$-like steady function arises. An adaptation zone in the surroundings of $q=0$ appears, where the solution passes from a Gaussian to a $\kappa$ function. The solution naturally adapts to the abrupt change in the problem parameters, connecting both zones in a smooth and stable way [61]. For a Gaussian initial function with $\langle 1\rangle=\left\langle q^{2}\right\rangle=1$ and $\langle q\rangle=0$, its evolution is drawn in figure 2.2a. A quasi-steady state is reached for $t=10$, where $f(q<0)$ has a Gaussian shape and $f(q>0)$ is a $\kappa$-like function. Close to $q=0$, an abrupt connection between both zones appears. This jump connects the two regions, but it does not introduce numerical instabilities. Another important result relies on the the evolution of $\langle 1\rangle$, [1], and $[q]$, figure $2.2 \mathrm{~b}$, being smooth and continuous in time. This means that even though parameters change abruptly, the solution evolves properly, as the analytical fine problem in [61]. On the other hand, the $f$ norm is conserved through the simulation, which means that no information is lost due to the discontinuity in the problem parameters. In resume, the sharp solutions found in this example indicates that situations as shocks or layers can be computed with the PIM, without introducing instabilities or requiring numerical additional viscosity.

Another important task is to extend these results to bound value problems. As it was presented in $[21,23,27]$, boundary conditions can be added to the one-dimensional time advancing scheme as a non-homogeneous term acting over the contour. Now, we focus on the semi-open one-dimensional problem where $q \in[0, \infty)$. The previous kappa problem with a reflecting boundary condition is studied. The procedure stated in [27] is used to introduce boundary conditions into the case presented in figure 2.1, augmented 


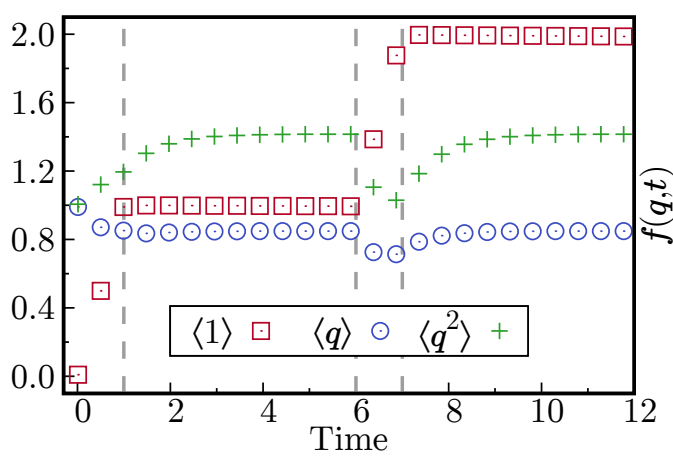

(a) Integral quantities

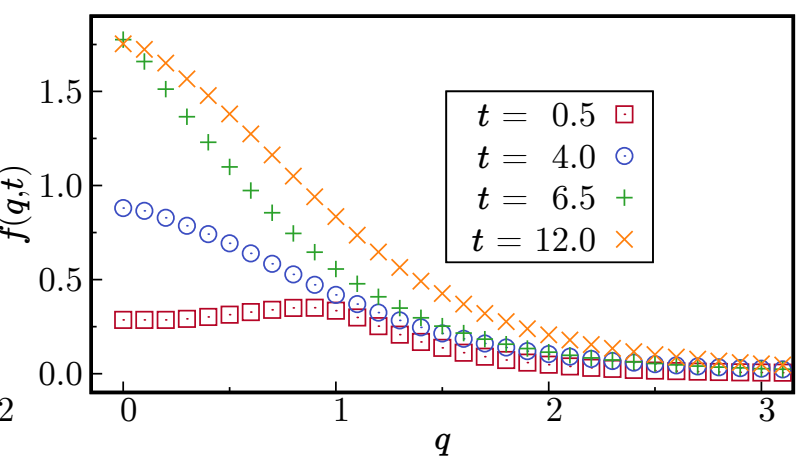

(b) Solution

Figure 2.3: Evolution of the bounded kappa problem. Norm varies only for two causes: non-homogeneous term or $\left.J[f]\right|_{q=0} \neq 0$.

in a semi-open domain with the boundary condition

$$
\left.J[f]\right|_{q=0}=\left\{\begin{array}{ll}
1 & \text { if } 6<t \leq 7 \\
0 & \text { otherwise }
\end{array} .\right.
$$

where $J[f]=D_{q} f-\partial D_{q q} f / \partial q$ is commonly known as the current operator. For a time of 12 non-dimensional units the system reaches a quasi-steady state after the boundary condition is modified, as shown in figure 2.3. A first relevant result states that problem norm, i.e., $\langle 1\rangle$ only changes when non-homogeneous or boundary terms are different from zero. Otherwise, this amount is conserved and the problem evolves driven only by the convective and diffusive parameters until a quasi-steady state is reached. The solution $f(q, t)$ fulfils the boundary condition at each time step and a change for $t=6.5$ appears, as the $f$ slope close to $q=0$ differs from 0 due to the action of the non-zero current. The adaptation to the boundary is smooth and its effect covers the full domain after a few time steps as the term $f_{B}$ exists for all $q \geq 0[23,27]$.

Another interesting boundary condition is the absorbing one in which $f(0, t)=0$. The propagator to represent this boundary condition can be calculated by standard method giving in [49]. The resulting short-time propagator becomes

$$
P_{\tau}\left(q ; q^{\prime}\right)=\frac{1}{\sqrt{4 \pi D_{q q}^{\prime} \tau}} \exp \left[-\frac{\left(q-q^{\prime}-\tau D_{q}^{\prime}\right)^{2}}{4 D_{q q}^{\prime} \tau}\right]\left(\exp \left[\frac{q q^{\prime}}{D_{q q}^{\prime} \tau}\right]-1\right),
$$

which is zero for any $q, q^{\prime}=0$. A basic example with constant drift and diffusive coefficients is carried out. For this problem, $D_{q q}=1$ and the convective force $D_{q}$ takes two values of opposite sign, -1 and 1 . The non-homogeneous term equals $\delta(q-1)$ and it is active through all the simulation. Figure 2.4 pictures the evolution of the norm and the solution for both cases. Two clearly distinguishable dynamics arise, for negative $D_{q}$ a quasi-steady situation is reached, for which $\langle 1\rangle$ remains constant, as the amount of $f$ introduced by the non-homogeneous term equilibrates with the norm being collected at $q=0$. However, when the force per unit mass is positive, the norm increases linearly, since the lost of norm is not compensated by drifting effects and loses at the absorbing boundary. 


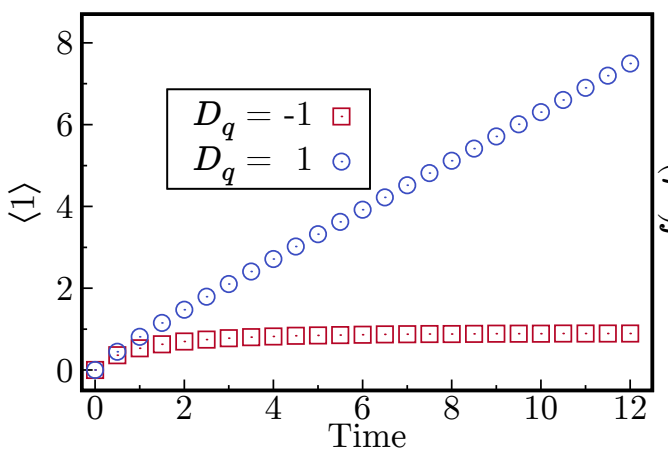

(a) Solution norm

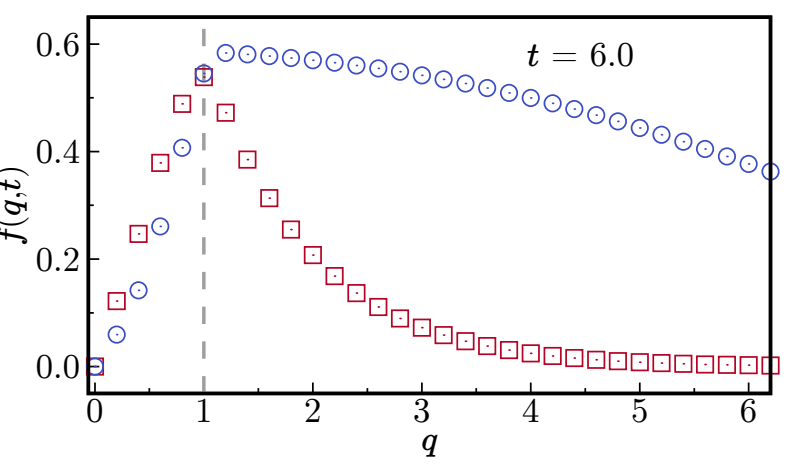

(b) Solution

Figure 2.4: Evolution of the bounded absorption problem. The parameter $D_{q}$ determines the system dynamics, but boundary effect always arise.

\subsubsection{Extension to $1+1 \mathrm{D}$ problems}

One important application of the drift-diffusion equation relies on the study of the solution evolution in a two dimensional phase space where the diffusive matrix is singular, i.e., $|\mathbb{D}|=0$. This means, diffusion only acts in one lineal variety, e.g., velocity space, while convective terms may appear in both directions. A general two dimensional equation for this process as a particular case of (2.10) is

$$
\frac{\partial f}{\partial t}+\frac{\partial}{\partial q_{1}} D_{q_{1}} f+\frac{\partial}{\partial q_{2}}\left[D_{q_{2}} f-\frac{\partial}{\partial q_{2}} D_{q_{2} q_{2}} f\right]=s,
$$

where $f=f\left(q_{1}, q_{2}, t\right), q_{1}$ and $q_{2}$ are two coordinates, with their corresponding drift parameters $D_{q_{1}}$ and $D_{q_{2}}$, and $D_{q_{2} q_{2}}$ is the diffusion in the $q_{2}$ direction given the diffusive matrix $\mathbb{D}$ is

$$
\mathbb{D}=\left(\begin{array}{cc}
0 & 0 \\
0 & D_{q_{2} q_{2}}
\end{array}\right) .
$$

For this equation, also known as the Klein-Kramers equation [62-64], many works have applied integral methods in an open space $[35,41,44,65]$ providing good result and capturing dynamics that classical schemes would miss, e.g., the parasite effective diffusion in the $q_{1} q_{1}$ and $q_{1} q_{2}$ transversal directions. In here, the PIM is used to solve this type of equations in an open domain. The bounded problem for this equation is still an open problem to solve even from analytical approaches, but some guidelines are given here to exemplify the complexity of this bounded problem.

A particular application of (2.30) is given to solve the Brownian motion [61,65-67] in a $x$ - $v$ open space, where $x$ represents the position and $v$ is the particular velocity in the $x$ direction. The partial differential equation that describes this motion is

$$
\frac{\partial f}{\partial t}+v \frac{\partial}{\partial x} f+\frac{\partial}{\partial v}\left[D_{v} f-\frac{\partial}{\partial v} D_{v v} f\right]=s
$$

where $D_{v}=-\gamma v+\frac{F_{e x t}}{m}$ is the convective parameter, $\gamma$ is a constant that plays the role of the characteristic problem frequency, $F_{\text {ext }}$ is the external force, $m$ is the particle mass 
and usually $D_{v v}=\gamma \frac{k T}{m}$ where $T$ is the temperature, although the procedure presented here can deal with drift-diffusion parameters of very different functional forms.

The easiest way to obtain a propagator for this problem, is to apply the Fourier transform to the auxiliary problem as in the work by J.M. Donoso and E. del Río [44]

$$
\frac{\partial P_{\tau}}{\partial \tau}+v \frac{\partial P_{\tau}}{\partial x}+\frac{\partial}{\partial v}\left[D_{v}^{\prime} P_{\tau}-\frac{\partial}{\partial v} D_{v v}^{\prime} P_{\tau}\right]=\delta\left(x-x^{\prime}\right) \delta\left(v-v^{\prime}\right),
$$

where $P_{\tau}=P_{\tau}\left(x, v ; x^{\prime}, v^{\prime}, \tau\right)$ is the short-time propagator by a double Fourier transformation as

$$
\mathfrak{F}\left(P_{\tau}\right)=\tilde{P}(\omega, k, \tau)=\int_{-\infty}^{\infty} \int_{-\infty}^{\infty} e^{-i \omega v-i k x} P_{\tau} d x d v
$$

reduces to

$$
\frac{\partial \tilde{P}}{\partial \tau}+\left[i D_{v}^{\prime}+\omega^{2} D_{v v}^{\prime}-k \frac{\partial}{\partial \omega}\right] \tilde{P}=e^{-i \omega v^{\prime}-i k x^{\prime}}
$$

that can be solved by the Method of Characteristics dealing with $\tilde{P}=1$ for $\tau=$ $0[41,44]$, given

$$
\begin{aligned}
\tilde{P}=\exp & {\left[-\frac{D_{v v}^{\prime}}{3}\left(k^{2} \tau^{3}+3 \omega k \tau^{2}+3 \omega^{2} \tau\right)\right.} \\
& \left.-i \omega\left(v^{\prime}+D_{v}^{\prime} \tau\right)-i k\left(x^{\prime}+v^{\prime} \tau+\frac{1}{2} D_{v}^{\prime} \tau\right)\right],
\end{aligned}
$$

which after Fourier inversion provides

$$
P_{\tau}\left(x, v ; x^{\prime}, v^{\prime}, \tau\right)=\frac{\sqrt{3}}{2 \pi \tau^{2} D_{v v}^{\prime}} \exp \left[-\frac{1}{D_{v v}^{\prime} \tau}\left(3 \frac{X^{2}}{\tau^{2}}+3 \frac{X V}{\tau}+V^{2}\right)\right],
$$

where $X=x-x^{\prime}-v^{\prime} \tau-\frac{1}{2} D_{v}^{\prime} \tau^{2}$ and $V=v-v^{\prime}-D_{v}^{\prime} \tau$. It is clear that different diffusion scales appear naturally in the problem. An interesting first feature is that even when the diffusion process only appears in the direction $v$, the resolution of the auxiliary problem shows that $D_{v v}$ produces diffusion in the directions $x$ and in $x-v$. Each diffusive effect evolves at different time scales $\left(\tau^{-1}, \tau^{-3}\right.$ and $\tau^{-2}$ respectively), a fact that is missed in a classical difference discretisations [44], which can give rise to non-physical evolutions of $f$. Thanks to the ability of the PIM to advance $f$ as a semi-analytical approximated solution, these scales, which really appear in an analytical Brownian motion solution, are properly represented. If these scales are not considered, differences in the time evolution and relaxation time of the solution can provide misleading results.

For $D_{v v}=1$ and $D_{v}=-v$, the results are presented in figure 2.5, where contour plot of $f(x, v, t)$ at different time frames and the corresponding three first order fluxes are pictured below. Starting from an histogram-like initial condition, the function evolves up to a Gaussian one. The diffusive scales in the $x-x$ and $x-v$ directions appear at much smaller rates than in the $D_{v v}$ direction. The evolution of $\langle 1\rangle$ reassembles a simple diffusive process where, without external force approaches zero for $t \rightarrow \infty$. The values $[1]$ and $[v]$ have an anti and symmetrical dynamics, respectively. 


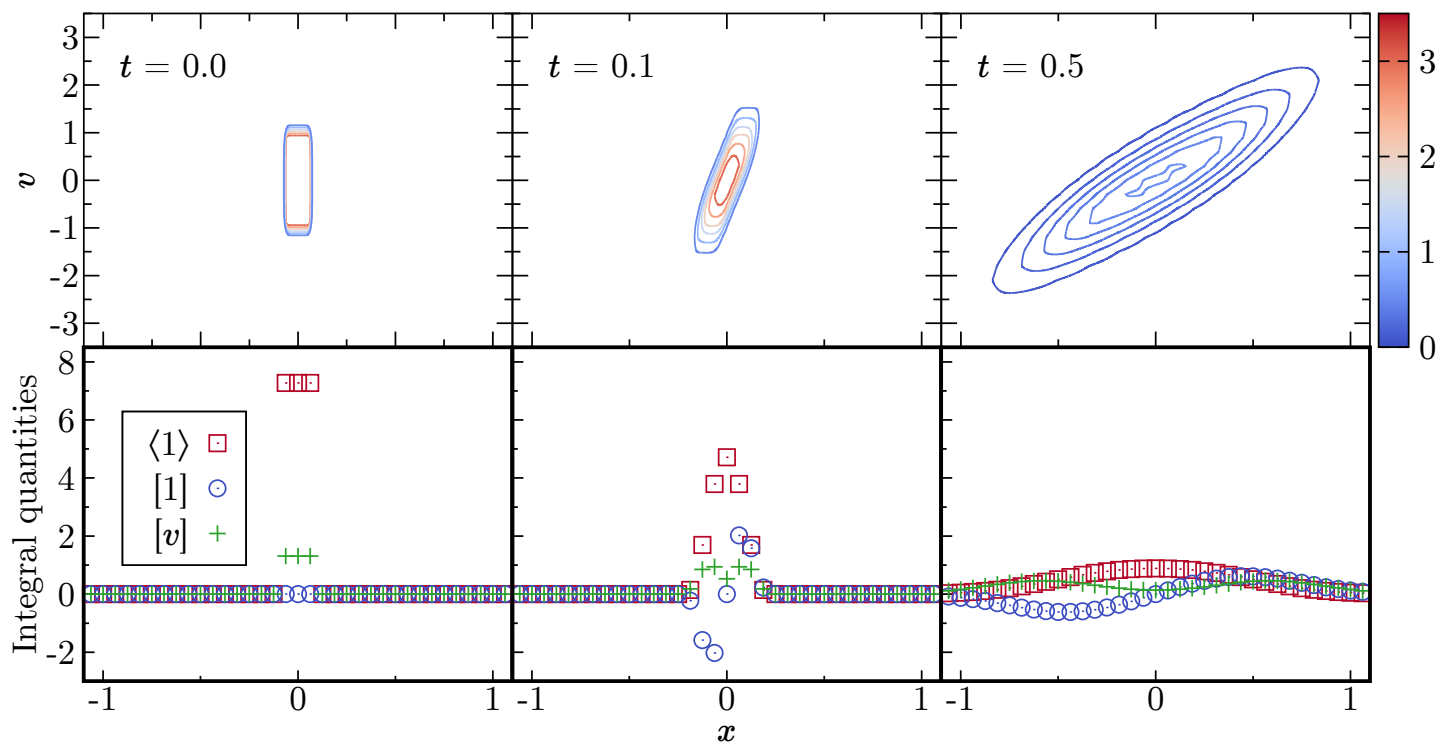

Figure 2.5: Evolution of $f(x, v, t)$ (top row), its norm and fluxes (bottom row).

The addition of boundary conditions to this problem is not straightforward. Due to the singular diffusion matrix, the boundary conditions that can be imposed in $x$ are limited. Let us assume a bounded domain in the region $x \in[0,1]$ and $v \in[-\infty, \infty]$. In typical kinetic problems, natural boundary conditions are imposed for the $v$ direction, i.e., $f$ and its derivatives vanished for $|v| \rightarrow \infty$, which means that the single boundary different from zero can be at $x=0$ and $x=1$. As seen in the example presented above, $f$ values at $v>0$ travel to the positive $x$ direction and vice-versa, due to the pure convective term of equation (2.32). This means,

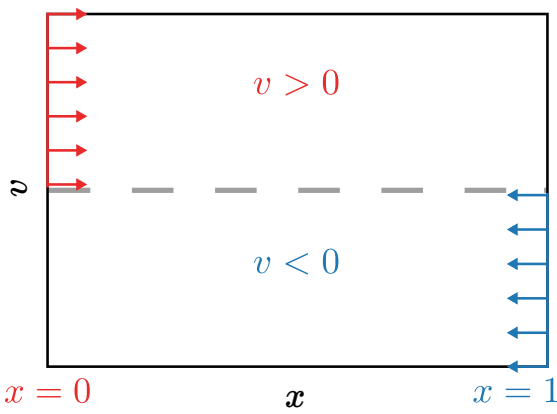

Figure 2.6: Zones where boundary conditions for the Klein-Kramers problem can be imposed are marked in red and blue for the $[0,1] \times[-\infty, \infty]$ $1 D 1 \mathrm{~V}$ domain. that the boundaries can only influence the problem inside $[0,1]$ if the normal flux is positive, i.e., if $\mathbf{n} \cdot \mathbf{v}>0[62,66-68]$. This is, at $x=0$, information with $v>0$ can be imposed as a boundary condition while $f(v<0,0, t)$ cannot be imposed as a boundary condition, but it must be modified by the imposed boundary for $v>0$. On the other hand, at $x=1$, only $f$ values with a negative $v$ can be imposed as boundary condition. This situation is resumed in figure 2.6, where the left boundary condition is represented in red and the right one is blue. Boundary conditions cannot be imposed in the black zones. Nevertheless, some works have been done to extend the boundary conditions that are applicable to this problem, as the one presented in reference [35]. The construction of more general short-time propagators suitable for this bounded problem is still a topic in research, as it is not a trivial task even for constant coefficients. 


\subsubsection{Extension to 2D problems}

The resolution of two and three-dimensional problems in the velocity space is of huge importance in the transport coefficient calculation for plasmas and gases $[13,18,43$, 69-74]. Unlike in the $1 \mathrm{D} 1 \mathrm{~V}$ case, here the diffusive matrix is usually non-singular. The general equation that describes these problems can be obtained by setting $N=2$ in (2.10) becoming

$$
\begin{aligned}
\frac{\partial f}{\partial t}= & -\frac{\partial}{\partial q_{1}}\left[D_{q_{1}} f-\frac{\partial}{\partial q_{1}} D_{11} f-\frac{\partial}{\partial q_{2}} D_{12}\right] \\
& -\frac{\partial}{\partial q_{2}}\left[D_{q_{2}} f-\frac{\partial}{\partial q_{2}} D_{22} f-\frac{\partial}{\partial q_{1}} D_{12}\right]+s,
\end{aligned}
$$

where $q_{i}$ is the velocity component in the $i$-direction and $D_{i}$ and $D_{i j}$ their associated drift-diffusion coefficients.

A relevant case for this work is the kinetic evolution of a distribution function in a parallel and perpendicular velocity space respect to a fixed force field. This space allows the inclusion of multiple collision terms, as Fokker-Planck-Landau or Boltzmann ones, for calculation of transport coefficients in plasmas in presence of an electromagnetic field. Assuming a three velocity space $\left(v_{x}, v_{y}\right.$ and $\left.v_{z}\right)$ in which a species of a plasma or a gas evolves in time, with space homogeneity. We can consider the system behaviour under the action of a external field, leading to the evolution in one privileged direction $\left(v_{z}\right)$. Let us call by parallel velocity $\left(v_{\|}\right)$to the one that matches this privileged direction and by perpendicular velocity to the composition of the other two $\left(v_{\perp}^{2}=v_{x}^{2}+v_{y}^{2}\right)$, resulting in an orthogonal change of variables. The remaining direction is identified by the angle $\phi$ as $v_{x}=v_{\perp} \cos \phi$ and $v_{y}=v_{\perp} \sin \phi$ in usual cylindrical coordinate system [59]. If we assume angular symmetry, the problem reduces to a two-dimensional one as

$$
\begin{aligned}
\frac{\partial F}{\partial t}= & -\frac{\partial}{\partial v_{\perp}}\left[\frac{D_{\phi \phi}}{v_{\perp}}+D_{\perp}-\frac{\partial}{\partial v_{\perp}} D_{\perp \perp}-\frac{\partial}{\partial v_{\|}} D_{\perp \|}\right] F \\
& -\frac{\partial}{\partial v_{\|}}\left[D_{\|}-\frac{\partial}{\partial D_{\|}} D_{\|\|}-\frac{\partial}{\partial v_{\perp}} D_{\perp \|}\right] F \\
& +2 \pi v_{\perp} s
\end{aligned}
$$

as it is stated in [59, Chapter 2] for general change of variables of the FP equation. Here $F\left(v_{\perp}, v_{\|}, t\right)=2 \pi v_{\perp} f\left(v_{\perp}, v_{\|}, t\right)$ is a simplified expression for the original solution which accounts for the geometry factor and integrated over $\phi$ direction. This equation must be solved in a $(-\infty, \infty) \times(0, \infty)$ domain for the parallel and perpendicular velocities, respectively. The propagator to solve (2.39) takes the form

$$
\begin{aligned}
P_{\tau}= & \frac{2 \pi v_{\perp}}{(4 \tau \pi)^{3 / 2} \sqrt{D_{t}^{\prime} D_{\phi \phi}^{*}}} \mathrm{i}_{0}\left(\frac{2 v_{\perp}\left(D_{\|\| \|}^{\prime} U+D_{\perp \|}^{\prime} W\right)}{4 D_{t}^{\prime} \tau}\right) \\
& \exp \left[-\frac{D_{\|\|}^{\prime}\left(v_{\perp}-U\right)^{2}-2 D_{\perp \|}^{\prime}\left(v_{\perp}-U\right) W+D_{\perp \perp}^{\prime} W^{2}}{4 \tau D_{t}^{\prime}}\right]
\end{aligned}
$$




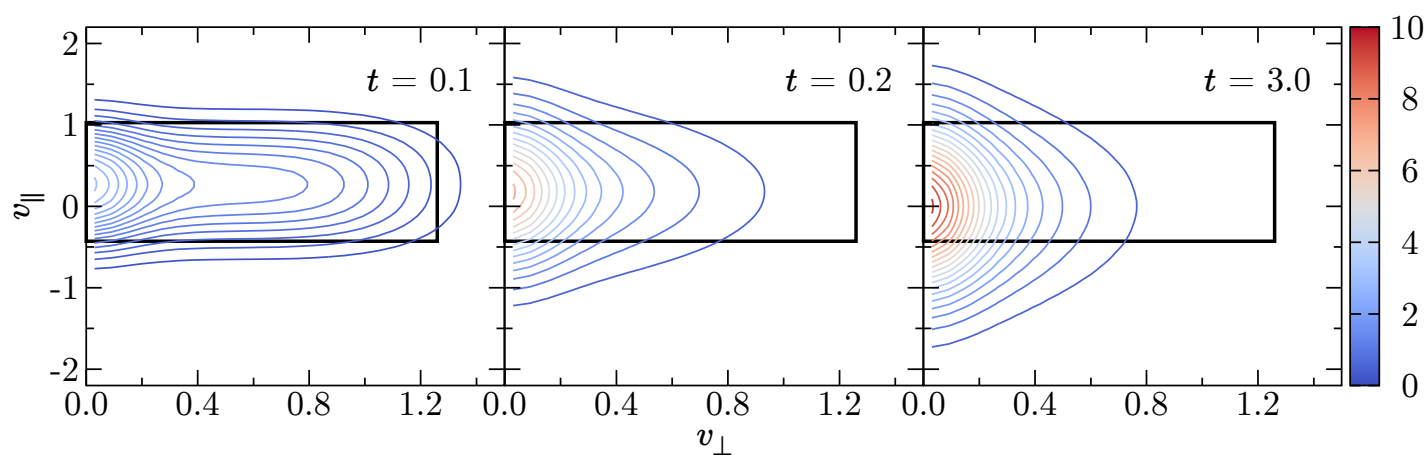

Figure 2.7: Evolution of $f=F / 2 \pi v_{\perp}$ starting from an abrupt initial condition until a Gaussian like solution naturally arise. Black solid line represents the initial condition.

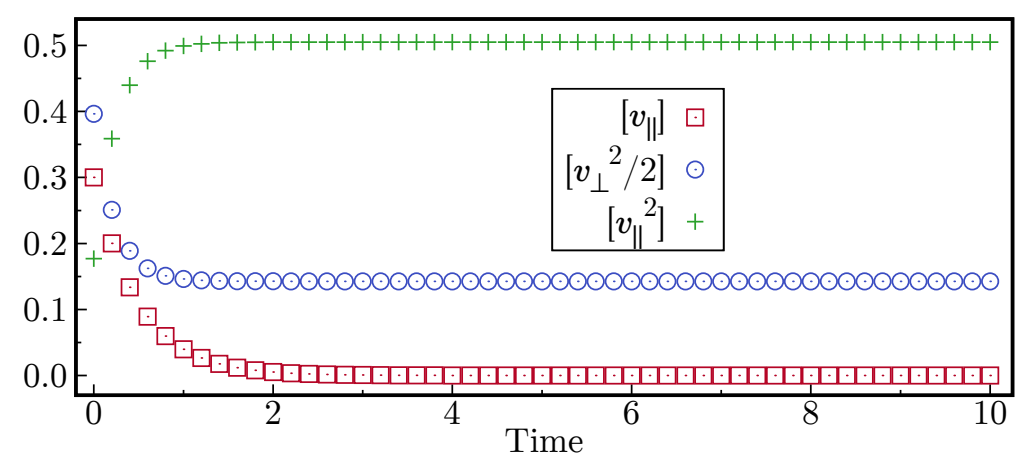

Figure 2.8: Evolution of the macroscopic quantities in both directions.

where $D_{t}^{\prime}$ is the determinant of the diffusion matrix, $U=v_{\perp}^{\prime}+A_{\perp}^{\prime} \tau, W=v_{\|}-v_{\|}^{\prime}-D_{\|}^{\prime} \tau$, $A_{\perp}^{\prime}=D_{\perp}^{\prime}+\left(D_{\phi \phi}^{\prime}-D_{\phi \phi}^{*}\right) / v_{\perp}, D_{\phi \phi}^{*}=D_{t}^{\prime} / D_{\|\|}^{\prime}$ and $\mathrm{i}_{0}(q)=\mathrm{I}_{0}(q) \exp (-q)$ where $\mathrm{I}_{0}$ is the modified Bessel function of order zero appearing, that accounts for the cylindrical geometry.

A simple test-case to check this propagator is selected for the convective parameters proportional to its velocity as $D_{i}=-2 v_{i}$ where $i=\perp, \|$ and $D_{\|\| \|}=1, D_{\perp \perp}=0.5$ and $D_{\perp \|}=D_{\phi \phi}=0$. Once again, the initial condition for $f$ is a histogram like function with non-zero mean parallel direction, i.e., $\left\langle v_{\|}\right\rangle \neq 0$ at $t=0$. It can be seen, figure 2.7, how the abrupt initial condition evolves into a Gaussian like distribution in a smooth way from the first iteration, without generating instabilities. The initial mean velocity $\left\langle v_{\|}\right\rangle$vanishes for large $t$, as figure 2.8 shows.

\subsubsection{Numerical hindrances}

The main problem to apply this method to drift-diffusive equations relies in the amount of points required to properly represent the propagator related to the intensity of the diffusion scale. As it has been explained, this effect influences, all the problem domain, however, in numerical problems, limitations due to machine precision and the grid width exist. Hence, small values of $\mathbb{D} \tau$ can result in an incorrect representation of the Gaussian 


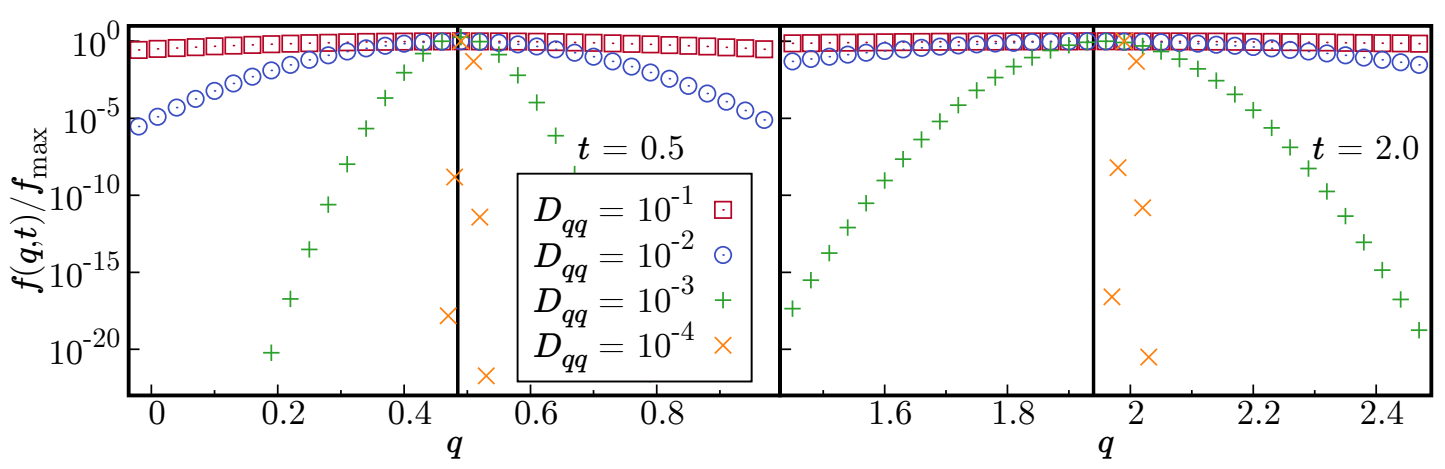

Figure 2.9: Evolution of the function $f(q, t)$, divided by its maximum $f_{\text {max }}$ for different values of $D_{q q}$. Black solid line depicts $D_{q} t$.

propagator, which may become closer to a numerical Dirac delta like function, meaning that diffusive effects are not properly represented. In resume, for small values of $\mathbb{D} \tau$ the propagator becomes very narrow and if there is no enough points in the numerical grid, the evolution of the solution is distorted. This is caused due to the numerical renormalisation of the propagator, required to ensure the conservation properties, as seen in [21], resulting in the propagator becoming a very thick Gaussian. This is specially important for the applications in multiple dimensions, where the propagator becomes a four-dimensional array, which limits the number of points that can be employed and, consequently, the diffusive and time scales that can be represented.

To show this difficulty, a case is presented for a one-dimensional drift-diffusive case with $D_{q}=0.97$ and $D_{q q}=10^{-k}$ where $k=1,2,3$ and 4 . All cases are executed with the same time-step $\tau=10^{-2}$ and $\Delta q=10^{-2}$. The drift coefficient is chosen this way to reduce the possibility that the maximum point of the Gaussian propagator coincide with a grid point. This way the maximum point of the propagator is not represented and, for low values of $D_{q q}$ the Gaussian shape will be distorted. The initial condition is a Dirac delta in $q=0$. In figure 2.9, the results are presented for $t=0.5$ and $t=2.0$. It can be easily checked how small diffusive parameters ( $k=3$ and 4$)$ move the solution's peak towards of the corresponding point $q_{\max }=D_{q} t$.

This representation lays out a serious difficulty for two dimensional phase-space $x-v$ problems, where the diffusion scale in the $x$ direction is $D_{v v} \tau^{3}$. This means that even for high values of $D_{v v}$, the propagator requires a large number of points in the $x$ direction to represent this small scale, as $\tau \ll 1$, otherwise, the evolution of the solution may become unstable and physicalness. In addition, the values for $\tau$ suitable for these problems get highly reduced, as small $\tau$ values result in situations where the parasite diffusions are too small to be properly represented. To see this, the test-case of the Brownian motion without external force starting from a Gaussian distribution as

$$
f_{0}(x, v)=\exp \left(-\frac{x^{2}}{0.1}\right) \frac{1}{\sqrt{2 \pi}} \exp \left(-\frac{v^{2}}{2}\right) .
$$

is solved with two values of time step: $\tau=0.1$ and 0.01 and $f(x, v, t)$ divided by their respective maximum values is drawn in figure 2.10. It can be seen how the solution is 


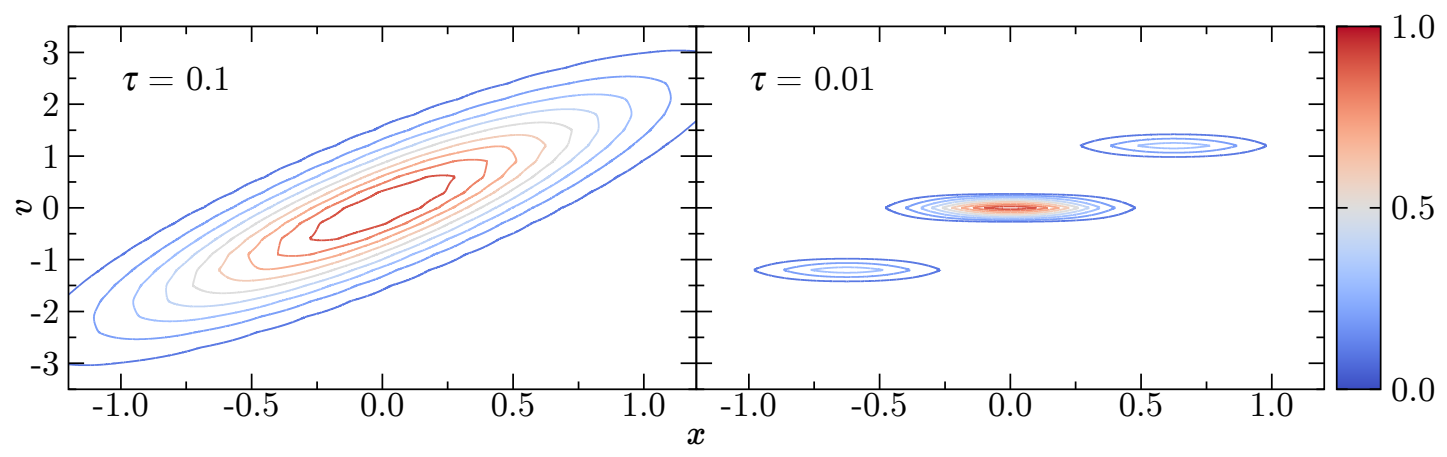

Figure 2.10: Solution $f(x, v, 0.5)$ divided by its maximum value for two values of the time step parameter.

disrupted for the smallest $\tau$ used, creating large areas where it is not well represented.

\subsubsection{Comparison with Finite Difference Methods and computational cost}

In spite of some numerical advantages and drawbacks of the PIM that have already been mentioned, this section pays attention to compare it and Finite Difference Methods (FDM). Solving partial differential equations with these well established methods require the discretisation of all derivative terms, a process that may cause non-physical behaviours and instabilities. To do so, the evolution of an abrupt initial condition under constant convective and diffusive parameters in a one-dimensional space is studied. The solution obtained by means of the PIM is presented along with two solutions obtained with FDM: one with an upwind and another with central discretisation schemes for the convective part. Diffusive gradients are always computed with a central discretisation scheme. To this aim, the simple problem

$$
\begin{aligned}
\frac{\partial f}{\partial t} & =-\frac{\partial}{\partial q}\left[D_{q} f-\frac{\partial D_{q q} f}{\partial q}\right] \\
f(q, 0)=f_{0}(q) & = \begin{cases}1 & \text { if }-0.5<q<0.5 \\
0 & \text { elsewhere }\end{cases}
\end{aligned}
$$

is solved in an unbounded space $q \in(-\infty, \infty)$ with natural boundary conditions. The parameters $D_{q}$ and $D_{q q}$ are constant and in this particular case equal 1 and 0.01 respectively. In these cases, the propagator is calculated at each time step, even though this is not necessary, since it provides a more general comparison of the computational cost, as drift-diffusive parameters usually evolve in time. For the three cases, a grid with $\Delta q=0.004$ is employed. In FDM cases, a small time parameter of $\tau=10^{-4}$ is required to avoid numerical instabilities. Meanwhile, PIM can employ a large time step of $\tau=0.1$. Even with this value of $\tau$, same time evolving solution as in the FDM is found, as seen in figure 2.11. In these figures, the time evolution of the solution obtained by the three numerical schemes is presented at three time frames. The three solutions 


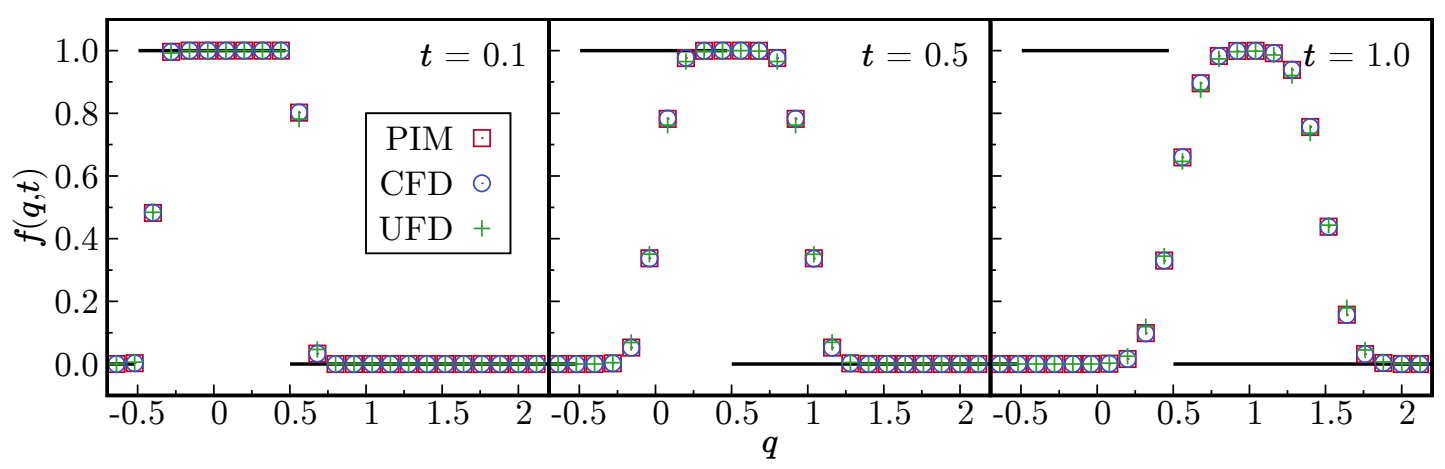

Figure 2.11: Evolution of an histogram-like initial condition solved by the PIM, central (CFD) and upwind (UFD) finite difference schemes with constant convective and diffusive parameters. Time step in PIM $(\tau=0.1)$ is larger than FDM $\left(\tau=10^{-4}\right)$.

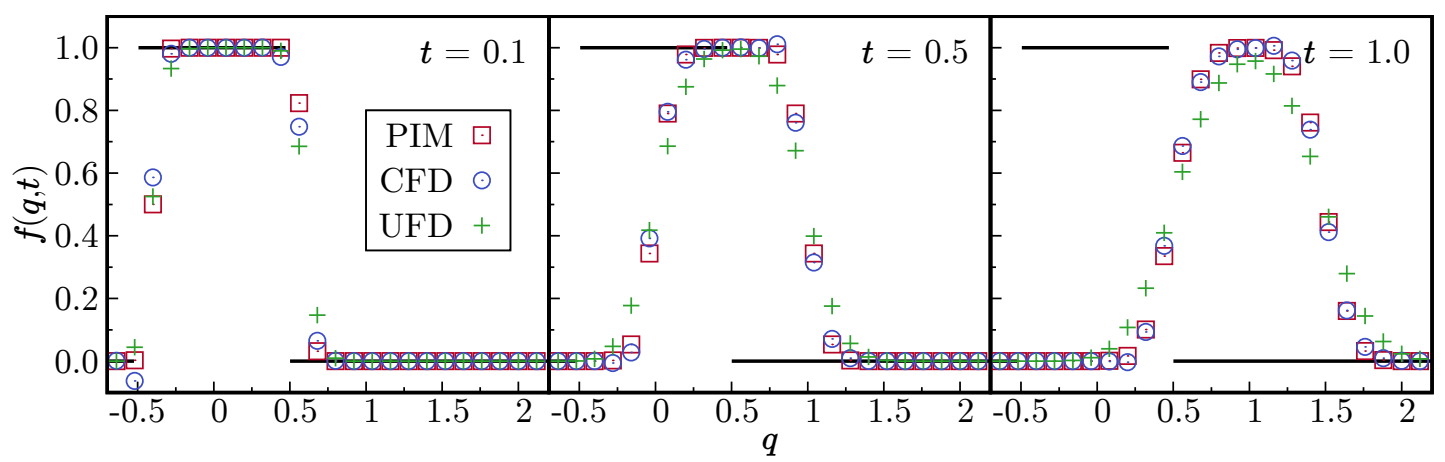

Figure 2.12: $\quad$ Same problem as in figure 2.11 but with $\Delta q=0.04$.

agree in their evolution, providing very similar results, with the solution UFD being a little below the other two as it introduces a small numerical viscosity. Now, if the space between grid points is increased by a factor 10 and the previous problem is solved again, notable differences arise. Figure 2.12 shows the function $f(q, t)$ at the same time frames as in figure 2.11 and significant differences among the three methods arise. For instance, some oscillations in the edges of the initial condition in the CFD solution appear, perturbing the evolution and giving some negative unrealistic $f$. These values tend to zero after some iterations due to viscous effects, but the negative solution, in this case, indicates a non-physical evolution for some time intervals. Furthermore, the solution with an upwind scheme presents significant numerical viscosity, as it can be seen for the high value tails and lower maximums. However, the solution obtained with the PIM keeps the positiveness of $f$ without generating oscillations or numerical viscosities and coincide with the case of a more refined grid.

It is important to remark that numerical instabilities do not necessary come from an insufficient number of grid points. In some situations, sharp gradients in the solution can generate numerical instabilities in FDM. Due to the lack of discretisation in the PIM, a more stable numerical solution is found, even in abrupt or discontinuous situations $[23,26]$. 


\begin{tabular}{ll}
\hline \multicolumn{1}{c}{ Component } & \multicolumn{1}{c}{ Model } \\
\hline Processor & Intel $^{\circledR}$ Core $^{\text {TM }}$ i7-4770K CPU @ 3.50 GHz \\
Memory & $2 \times 8192$ MB DDR3 1600 MHz \\
Motherboard & ASUSTeK ${ }^{\circledR}$ B85-PLUS \\
Hard disk & Samsung $^{\circledR}$ SSD 840 \\
Graphics card & NVIDIA $^{\circledR}$ GeForce ${ }^{\circledR}$ GTX 660 \\
Power supply & Thermaltake $^{\circledR}$ LT-600P \\
Operating System & Kubuntu 14.04.4 LTS \\
Compiler & GNU Fortran 4.8.4 \\
\hline
\end{tabular}

Table 2.1: Specifications of the computer employed to compare computational times among numerical methods.

\begin{tabular}{lll}
\hline Method & \multicolumn{2}{c}{ Time } \\
\hline \multirow{2}{*}{ Central } & Average time per iteration (ms) & 0.0464 \\
& Total time (s) & 0.5023 \\
\multirow{2}{*}{ Upwind } & Average time per iteration (ms) & 0.0389 \\
& Total time (s) & 0.4187 \\
\multirow{2}{*}{ PIM } & Average time per iteration (ms) & 31.139 \\
& Total time (s) & 0.3115 \\
\hline
\end{tabular}

Table 2.2: $\quad$ Computational times per iteration and total time for each method employed to solve (2.43) with $\Delta q=0.004$.

With respect the computation time, we have tested the different wall clock times ${ }^{1}$ required to compute the solution from the case above. The main resources consuming task in the PIM is the calculation of the short-time propagator and its re-normalization. The calculation of the next step solution can be usually simplified and its computational time can be reduced by parallelisation, as explained in appendix B.1. To compare the computation time of the different methods, the computer which specifications are presented in table 2.1 is employed to solve all simulations. The results in table 2.2 show how methods based on finite differences beat the PIM time per iteration. However, the total time is lower in the PIM due to the reduced number of iterations required to simulate the same time. Beside this, we have to be aware that more complex nonlinearities may require a lower $\tau$ for the FDM, increasing the number of iterations, while PIM could still use a relatively large time step.

In addition to the high computational cost per iteration of the PIM, the propagator is

${ }^{1}$ Wall clock time refers to the time measured by a clock, i.e., the physical time that has passed. 
a multidimensional array $2 N$ (where $\mathrm{N}$ is the problem dimension) that must be saved in memory to integrate over the grid. Although this can be avoided by employing an on the fly calculation of $P_{\tau}$ in the same loop as the advancing scheme, which will be studied in future works.

\subsection{Purely convective equations}

A quite usual type of equations that appears in kinetic and fluid descriptions of plasmas is the pure convective case of (2.10), whose general form can be cast into

$$
\begin{gathered}
\frac{\partial f}{\partial t}+\sum_{i}^{N} \frac{\partial}{\partial q_{i}} D_{i} f=s \\
f(\partial \mathfrak{D}, t) g(f, \mathbf{q}, t) \\
f(\mathbf{q}, 0) f_{0}(\mathbf{q}) .
\end{gathered}
$$

where $g$ is the function value at the boundary $\partial \mathfrak{D}$. Since this is a first order problem, only Dirichlet conditions are allowed at the boundary as it happens in the Brownian motion studied above. Moreover, to impose such a boundary condition, $\mathbf{n} \cdot \mathbf{D}>0$ must be fulfilled, where $\mathbf{n}$ is the outwards vector normal to the boundary. In classical numerical methods, the resolution of these equations requires, usually, the addition of numerical viscosity to avoid oscillations and instabilities, perturbing the transient solution. In this section, the PIM is extended to solve these equations providing stable and physically meaningful solutions that do not require any discretsation or additional viscosity. This allows the resolution of these equations from a new point of view, which results in new interpretations and applications to solve theoretical models.

It is expected that the PIM can be applied in the same way as for Fokker-Planck equations to obtain a time evolving solution, although a new short-time propagator $P_{\tau}$ is required. A simple way to calculate such a short-time propagator for the multidimensional open space is to calculate the limit of (2.13) when the diffusion matrix determinant $|\mathbb{D}|$, as well as each of its components $D_{i j}$, goes to zero. This results in a multidimensional Dirac delta as a degenerated Gaussian multivariable

$$
P_{\tau}\left(\mathbf{q}, \mathbf{q}^{\prime}, \tau\right)=\delta\left(q_{i}-q_{i}^{\prime}-D_{i}^{\prime} \tau\right)
$$

where $q_{i}$ are the components of the coordinate vector $\mathbf{q}$ in the direction $i$. The numerical implementation of this Dirac delta function and the resolution of some general cases is dealt in the next sections. 


\subsubsection{The non-diffusive one-dimensional problem}

When (2.46) is reduced to a one-dimensional space, becomes

$$
\begin{aligned}
\frac{\partial f}{\partial t}+\frac{\partial}{\partial q} D_{q} f & =s \\
f\left(q_{b}, t\right) & =g(f, q, t) \\
f(q, 0) & =f_{0}(q) .
\end{aligned}
$$

where $q_{b}$ is the boundary point and $D_{q}$ is the drift parameter. Whereas this problem seems to be quite simple, its numerical resolution with classical methods becomes a difficult task to avoid unstable solutions if discontinuities, high slope zones, or nonlinearities appear. To apply the PIM, the same procedure used for Fokker-Planck equations is employed, the general propagator (2.47) is computed only in the onedimensional open space, as

$$
P_{\tau}\left(q ; q^{\prime}, \tau\right)=\delta\left(q-q^{\prime}-D_{q}^{\prime} \tau\right)
$$

With this, the time advancing scheme is equivalent to (2.21), because it is independent of the propagator shape. To solve this problem numerically, an accurate representation of the Dirac delta must be employed, in this work, the expression (2.26) is proposed. Nevertheless, the evolution of this problem numerically presents an important issue that must be addressed.

In contrast with the drift-diffusion equation, where $f^{\prime}$ from source coordinate $q^{\prime}$ spreads to all the domain, here the $f$ value moves from point to point in a dynamics controlled by the convective force $D_{q}$. Let us assume we have $f^{\prime} \neq 0$ at point $q_{i^{\prime}}$ time $t^{\prime}$ where $i^{\prime}$ designates a generic source point in the grid. Due to the convective force $D_{i^{\prime}}$, this value moves towards $q_{i}=q_{i^{\prime}}+D_{i^{\prime}} \tau$ in a short time $\tau$. Let us assume that an equally-spaced grid has been created where $q_{i^{\prime}}$ and $q_{i^{\prime}+1}$ points are separated by a constant distance of $\Delta q$. If the value

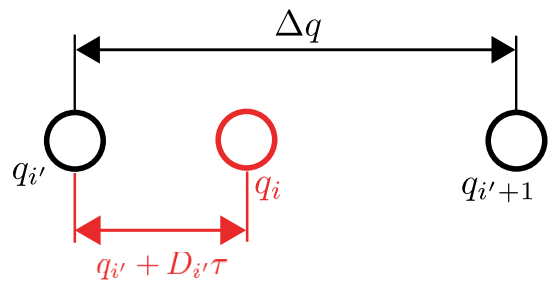

Figure 2.13: A situation where $q_{i^{\prime}+1}$ does not match $q_{i^{\prime}}+D_{i^{\prime}} \tau$. A new point at $q_{i^{\prime}}+D_{i^{\prime}} \tau$ is required to properly represent the solution motion. of $f$ at $q_{i^{\prime}}$ is advanced with the delta propagator, the corresponding field point is only modified if $\Delta q=D_{i^{\prime}} \tau$, which is unlikely except for some reduced values of $D_{q}$. To solve this, a displacement of the grid to follow the evolution of $D_{i^{\prime}}$ is proposed. To allow the propagator to properly represent the motion of $f$, a new set of coordinates $q_{i}$ is computed at each time step of the simulation as

$$
q_{i}=q_{i^{\prime}}+D_{i^{\prime}} \tau
$$

which ensures that a point exists where the information from source variables moves to the field coordinates perfectly. As figure 2.13 shows, if a fixed mesh is employed, source $f^{\prime}$ does not reach a corresponding field grid point, and its value is lost. To ensure that 


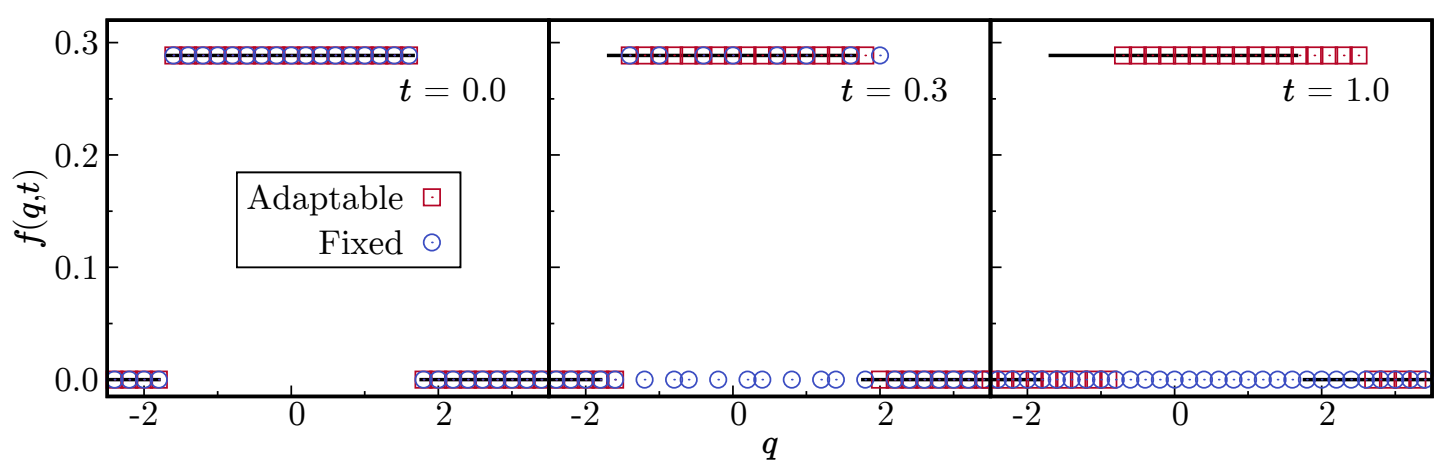

Figure 2.14: Resolution of a simple convective problem with a fixed mesh (blue circles) and the one recalculated by equation (2.52) (red squares).

points at the proper position exit, a new grid is created, using the field coordinates based on the source coordinates as stated above. So now, numerical grid moves and follows $D_{q}^{\prime}$, meaning that each grid point has an independent evolution, representing the local phenomena of the convective force. Differences between convective and diffusive processes are clearly understood in this situation. When diffusion appears, the value at a source point is transmitted, theoretically, to all the numerical domain, however, convection is a local phenomena that moves the solution as a block. This is also a reason of why FDM have issues dealing with this type of equations. In those methods the derivative at point $i$ is computed with the adjacent ones, which means that the value in one point is polluted by the contiguous point values, whereas the PIM only account for the local dynamics of each individual point.

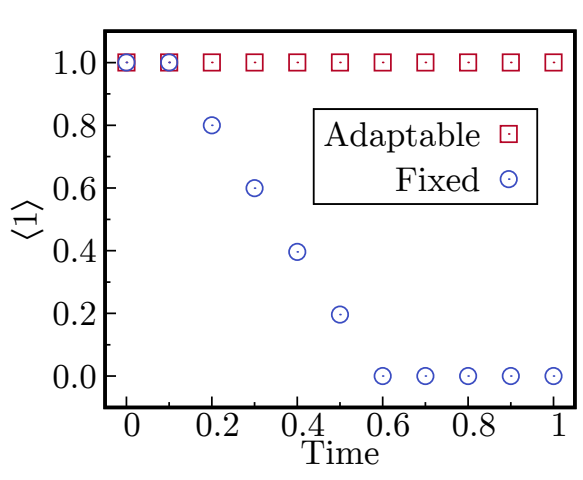

Figure 2.15: Norm of the pure convective case with fixed and adaptable meshes.

A simple convective problem with a constant force is compared for the cases with the mesh recalculated at each time step and a fixed grid. The initial step condition, figure 2.14, is lost with the fixed mesh until completely vanishes. Meanwhile, with the recalculation of the grid, $f(q, t)$ moves with the constant drift, what is more, the solution presents no oscillations, even in the edges of the initial condition. With classical numerical methods perturbations as Gibbs or numerical viscosity may produce non-physical solutions due to the inclusion of terms that do not appear in the original problem, as seen in section 2.3.4. In addition, the norm is conserved for the adaptable grid case, as can be seen in figure 2.15.

Another case is shown here, the so-called Burgers' equation [75-77]

$$
\frac{\partial f}{\partial t}+f \frac{\partial f}{\partial q}-D_{q q} \frac{\partial^{2} f}{\partial q^{2}}
$$

which becomes the inviscid Burgers' equation for $D_{q q} \rightarrow 0$. In the study of this problem it is common to add a constant diffusion, usually of small value, to explore the nature 


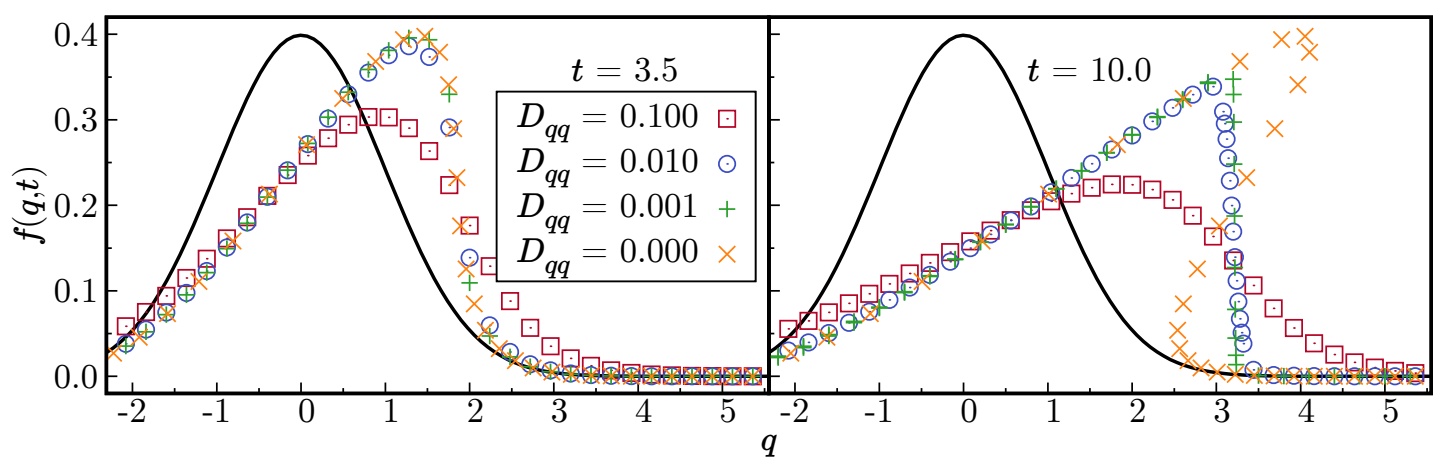

(a) Pre-shock

(b) After-shock

Figure 2.16: Solution of the Burgers equation for different values of $D_{q q}$ at $t=3.50$ and $t=10.0$. The initial condition is represented by a black line.

of solutions, since a shock may appear in time, depending on the initial condition $f_{0}(q)$ and the diffusion value $D_{q q}$. This shock is usually difficult to capture numerically with classical methods, and two possibilities may occur: oscillations appear or a numerical viscosity must be introduced to smooth the solution close to the shock. Both numerical phenomena obviously perturb the final solution in a non-physical way, leading to results that may be considerably different when this or similar equations are used, e.g., in the Navier-Stokes set of equations, where the balance equation for momentum has a similar shape as the viscous Burger's one. However, if $D_{q q}$ is zero, no shock should appear and the evolution of the problem should continue unperturbed. In our numerical problem solved with the PIM a mesh of length 20 with an initial Gaussian function is employed. To found the different behaviours, a variety of diffusion parameters are used, taking the values: $0.1,0.01,0.001$, and 0 . The results before the formation of the shock (figure 2.16a) and after (figure 2.16b) are presented. It can be seen how the shock intensity slightly increases as diffusion reduces. For the highest diffusion, 0.1 , no relevant shock appears and the solution remains close to a drifting Gaussian function. This is because viscous effect dominates over the convective one, reducing the intensity of the shock until it is indistinguishable. If we reduce $D_{q q}$, the shock become thinner, and the solution is dominated by the convective force. However, the shock always appears as far as $D_{q q}>0$ is fulfilled. Also, it can be observed how due to the diffusive effect, the maximum point of the solution decreases respect to its initial value as time advances. But if no diffusive term is accounted for, no shock materialises, and a situation where a point with high value of $f$ overcomes those with a lower value is reached. Even if this type of solutions have no physical meaning, it is important to stress out that small values of diffusion, used in the numerical resolution of this equation to avoid oscillations, can perturb the evolution of the solution. Moreover, the maximum value of the initial condition is kept constant if $D_{q q}=0$, as no diffusion effect reduces it. Nevertheless, evolutions before the shock for non-viscous and low viscosity values are very similar.

Particularly for this numerical resolution of the Burgers' equation, there is a bifurcation in the dynamics of the system at $D_{q q}=0$, i.e., the evolution of the inviscid case 
cannot be predicted, even inferred, with a very small viscosity. A similar behaviour can occur when Euler equations are employed to describe the evolution of a system from a fluid point of view (or Vlasov, in kinetic models). When a shock or a boundary layer appears, viscosity (collisional) effects become important and are needed to describe these phenomena so, an Euler (Vlasov) description will fail in these cases. Viscosity (collisional) effects can become important in some instances even if their are very small.

A final illustration, involving a non-homogeneous term, is studied. To account for this effect, an expression for the integral in time of the propagator is required. This can be done with a simple approximation $Q_{\tau} \simeq \tau P_{\tau}$ or by solving the analytical integral

$$
Q_{\tau}\left(q ; q^{\prime}, \tau\right)=\int_{0}^{\tau} P_{\tau}\left(q ; q^{\prime}, \tau^{\prime}\right) \mathrm{d} \tau^{\prime}
$$

which for the non-diffusive open-space propagator becomes

$$
Q_{\tau}\left(q ; q^{\prime}, \tau\right)=\frac{1}{D_{q}^{\prime}}\left[H\left(q-q^{\prime}-D_{q}^{\prime} \tau\right)-H\left(q-q^{\prime}\right)\right] .
$$

where $H$ is the Heaviside function. For $D_{q}^{\prime} \rightarrow 0$, an inconsistency appears, which requires a recalculation of $Q_{\tau}$ as

$$
\left.Q_{\tau}\left(q ; q^{\prime}, \tau\right)\right|_{D_{q}^{\prime}=0}=\delta\left(q-q^{\prime}\right) \tau
$$

A case can be extrapolated to the problem of a non-homogeneous term and a constant force. Let us assume the following general problem

$$
\begin{aligned}
\frac{\partial f}{\partial t}+\gamma \frac{\partial f}{\partial v} & =-\left(f-f_{\text {Gau }}\right) \\
f(q, 0) & =f_{0}(q)
\end{aligned}
$$

where $\gamma$ is a constant and $f_{\text {Gau }}=(2 \pi)^{-\frac{1}{2}} \exp \left[-\frac{q^{2}}{2}\right]$ is a Gaussian distribution. During all the simulations, an acceleration $\gamma=1$ moves the solution to increasing positive $q$ at a constant rate. An analytical solution exists for this particular problem

$$
\begin{aligned}
f(q, t)=\frac{\sqrt{\pi}}{2 \gamma} e^{\frac{-4 \gamma q+4 \gamma+1}{4 \gamma^{2}}} & {\left[\operatorname{erf}\left(\gamma t+\frac{1}{2 \gamma}-q+1\right)\right.} \\
& \left.-\operatorname{erf}\left(\frac{1}{2 \gamma}-q+1\right)\right]+e^{-t} f_{0}(q-\gamma t),
\end{aligned}
$$

where erf () is the usual error function defined as

$$
\operatorname{erf}(x)=\frac{1}{\sqrt{\pi}} \int_{-x}^{x} \exp \left(-t^{2}\right) \mathrm{d} t,
$$

and the initial condition is, again, the drawer function

$$
f_{0}(q)= \begin{cases}0.5 & \text { if }-1 \leq q \leq 1 \\ 0 & \text { elsewhere }\end{cases}
$$




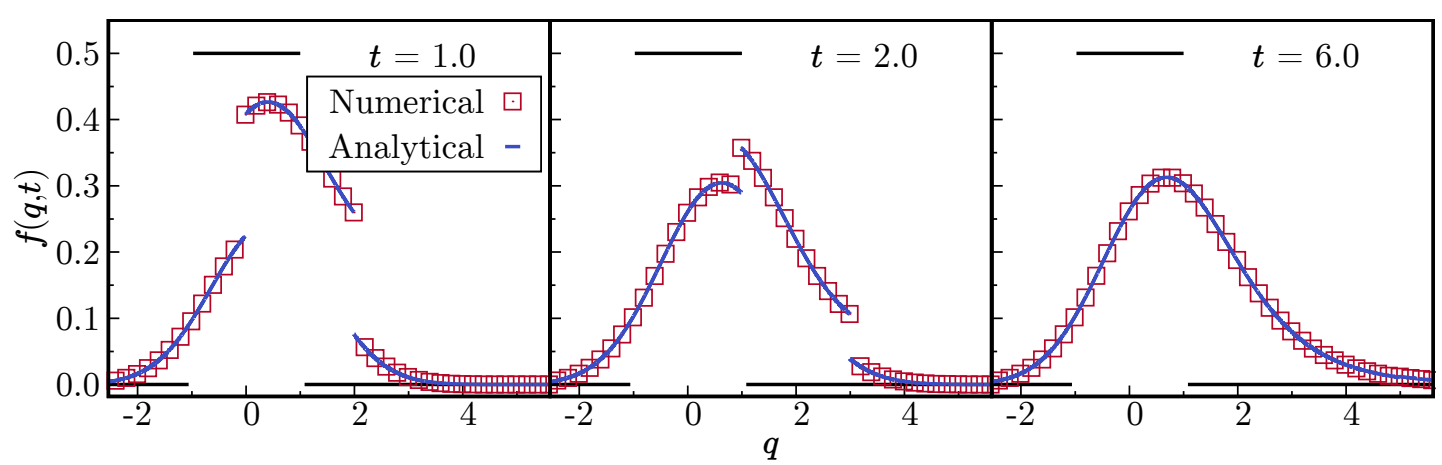

Figure 2.17: Evolution of $f(q, t)$ for numerical (red squares) and analytical solutions (blue line). Discontinuity is preserved in the numerical solution without perturbing it. Initial condition is drawn as a black solid line.

With this particular $f_{0}(q)$, the analytical solution of the problem keeps the initial discontinuity for a certain time interval, and ends up going away due to the exponential term in front of it in (2.58). An accurate numerical resolution of this problem should keep this discontinuity in the time evolution without introducing any viscous terms or generating oscillations. In the solution presented in figure 2.17 , it can be seen how the numerical solution obtained by means of PIM keeps the discontinuity and agrees with the analytical one. Classical numerical schemes would fail to represent this discontinuity from the very first iteration due to the need of numerically discretise $D_{q} f$, which generates a Gibbs phenomena or adds non-real viscosity. Another important difference between the PIM and the classical finite differences method is that the propagator works for any sign of $D_{q}$. In finite differences, $\operatorname{sgn}\left(D_{q}\right)$ determines the right discretisation scheme, if upwind method is employed, that avoid non-physical evolutions. This can be a problem if, for example, cases with a non-linear $D_{q}$ are solved, and it can limit the utilisation of upwind schemes, leading to problems solvable only by central differences schemes, which usually introduce numerical oscillations and instabilities.

\subsubsection{Extension to $2 \mathrm{D}$ problems}

If the diffusive contribution is removed from (2.30), a two-dimensional purely convective problem is obtained in the form

$$
\frac{\partial f}{\partial t}+\frac{\partial}{\partial q_{1}} D_{q_{1}} f+\frac{\partial}{\partial q_{2}} D_{q_{2}} f=s .
$$

This type of equations can be solved with the PIM as shown with the one-dimensional case of the previous section. The elimination of the diffusion part in (2.30) leaves this problem as a general $2 \mathrm{D}$ equation, so there is no difference in dealing with a $2 \mathrm{D}$ position space or a $x-v$ phase-space as no singularity of the stress tensor can appear. However, the non-fixed grid required to properly represent the evolution of $f$ imposes some extra constrains. The generic propagator for the open space is the product of Dirac delta functions

$$
P_{\tau}\left(q_{1}, q_{2} ; q_{1}^{\prime}, q_{2}^{\prime}, \tau\right)=\delta\left(q_{1}-q_{1}^{\prime}-D_{q_{1}}^{\prime} \tau\right) \delta\left(q_{2}-q_{2}^{\prime}-D_{q_{2}}^{\prime} \tau\right)
$$




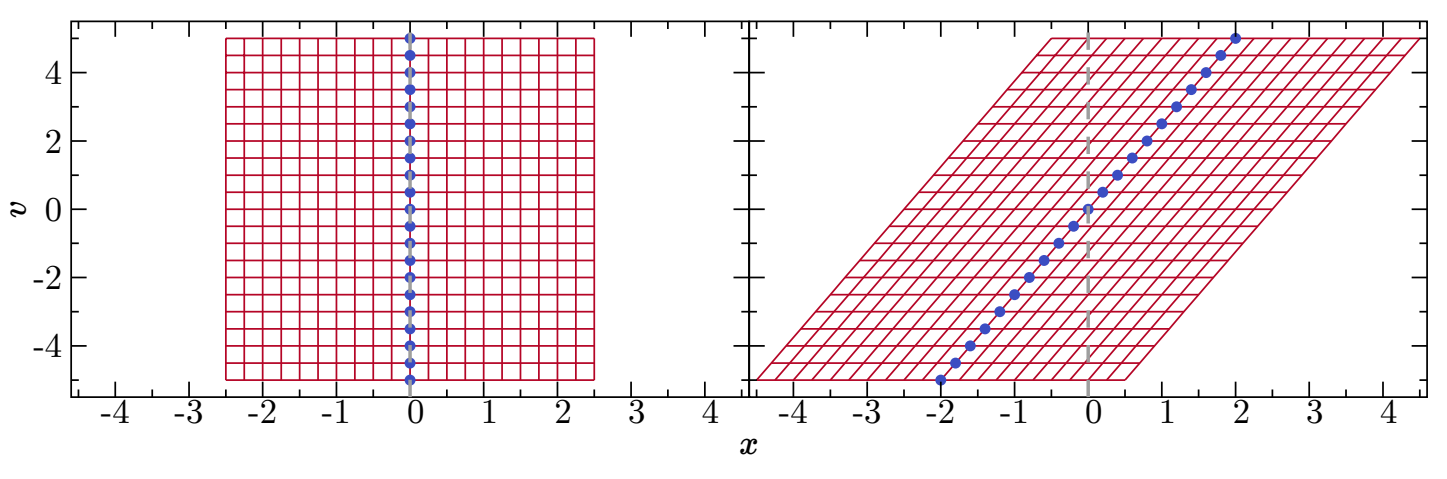

(a) $t=0.0$

(b) $t=0.5$

Figure 2.18: Grid displacement (in red) in a $1 D 1 V x-v$ space for $F=0$. Points corresponding to index $x=0$ at the first iteration are marked with blue filled circles.

similar to the multiplicative Gaussian probability density in the two-dimensional diffusive case.

An special application for the phase-space $x-v$, similar to the diffusive case, is presented here, being the convective force in the $x$ direction its associated velocity $v$. As for the one-dimensional convective problem, the mesh needs to move at each time step to ensure that the time evolving solution is well represented numerically. But now, the mesh moves in two directions, this means that the value of $f$ at point $\left(x^{\prime}, v^{\prime}\right)$ moves to $\left(x^{\prime}+v^{\prime} \tau, v^{\prime}+D_{v}^{\prime} \tau\right)$ in a small time $\tau$. This adds some difficulty to compute the macroscopic quantities of $f(x, v, t)$.

Figure 2.18 shows that the indexes corresponding to $x=0$, blue filled circles, at an initial time move and are not valid to integrate $f$ along $v$ at $x=0$, dashed grey line, for $t>0$. If the integration along the blue points is performed, the expected value is, in general, different as the one obtained by an integration over the dashed grey line as the values of $f(x, v, t)$ are different. In this work, an auxiliary fixed grid is proposed to interpolate the function and allow an easy calculation of its integral quantities. The subroutine for the interpolation can be found in section B.5. This of course, increments the computational time and reduces the accuracy of the solution, but the results are good enough to analyse some plasma dynamics, as presented in section 3.3.1. Nevertheless, any other method that allows the integration of the solution for all the positions $x$ should be valid.

The test-case of equation (2.61) solved by PIM with the interpolation of the solution in a fixed grid is presented now. An external dimensionless force of constant magnitude but opposite sense of displacement as $-\operatorname{sgn}(x)$ is applied, meaning $f$ is slowed down for $x \geq 0$ and is accelerated positively for $x>0$. For the histogram initial condition centred at the origin the evolution of both, the function and its interpolation, appears in figure 2.19. This diffusionless case with $s=0$ shows a solution keeping its initial value only subjected to convective motion in the $v$ and $x$ directions. The interpolated function (bottom row) shows some errors close to the abrupt change that can perturb 


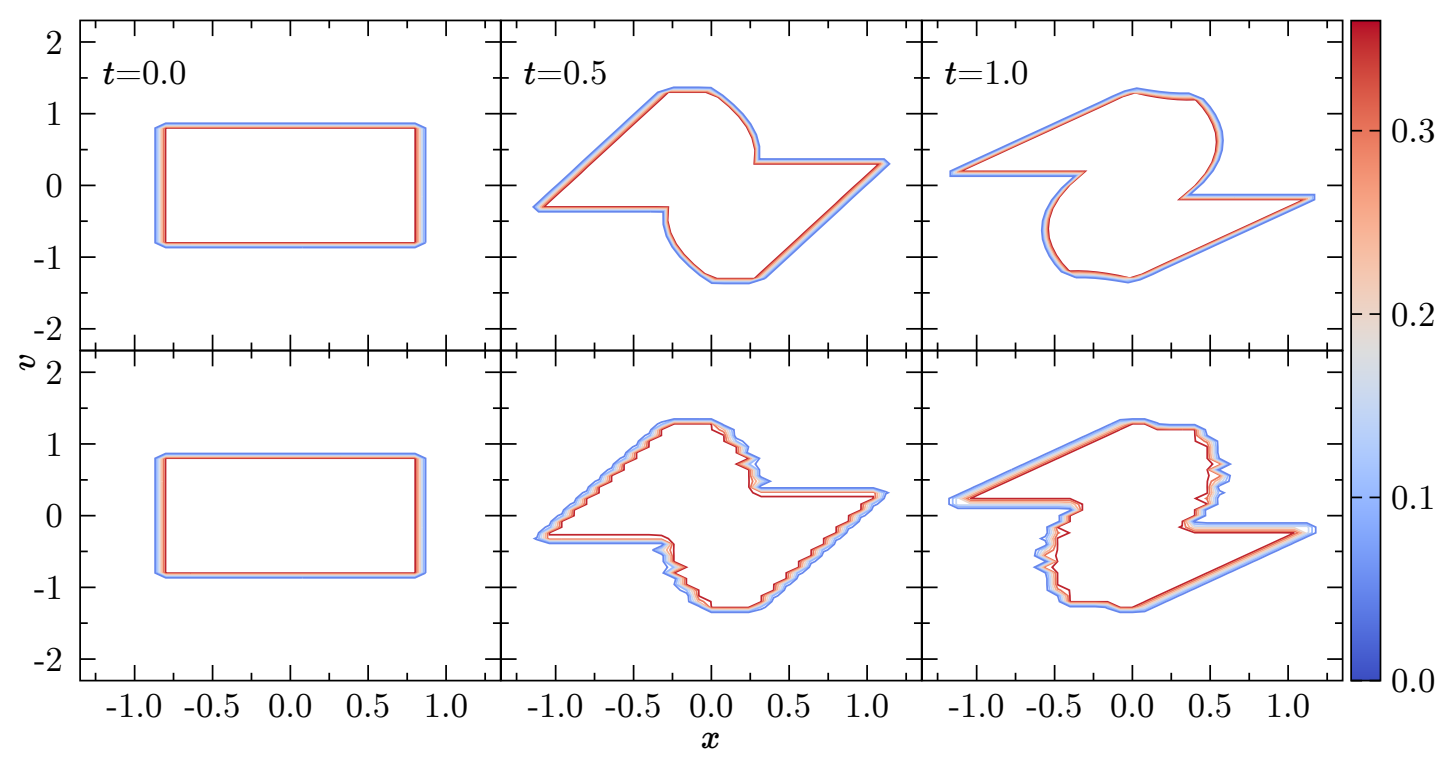

Figure 2.19: Evolution of $f(x, v x t)$ (top row) and its interpolation (bottom row) for an histogram initial condition and an external acceleration of $-\operatorname{sgn}(x)$.

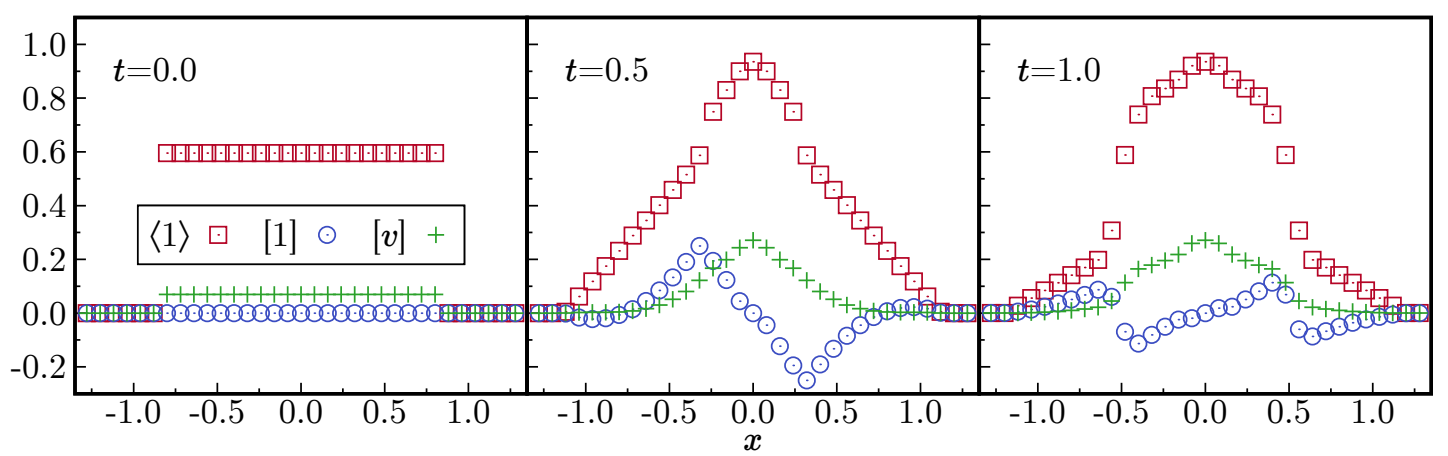

Figure 2.20: Norm and fluxes of order 0 and 1 of the interpolated function from figure 2.19.

the calculation of $f$ and its integral quantities. It is expected that the use of of more refined grids improves the solution and its interpolation, but it increases the computational cost. Nevertheless, the solution evolves smoothly by keeping discontinuities without inducing oscillations or instabilities.

The integral quantities $\langle 1\rangle$ and [v], see figure 2.20, are symmetrical whereas [1] is antisymmetrical. The abrupt changes of $\langle 1\rangle$ around $x= \pm 0.5$ coincide with the point where $f(x, v, t)$ suddenly changes. Even with the small errors of the interpolated solutions, the integrals of $f$ are well represented, but we are not in position to assert that this will also happen if, for example, the force or non-homogeneous term depend on these values.

If the drift represents a friction force $D_{v}=-x$, i.e., the Hooke's law, a periodical dynamics around $(x, v)=(0,0)$ is found, figure 2.21, with an approximate period of $\pi / 2$, as theoretically given. This means that no numerical viscosity or oscillations are spuriously introduced in the problem as the exact same solution is recovered after a 


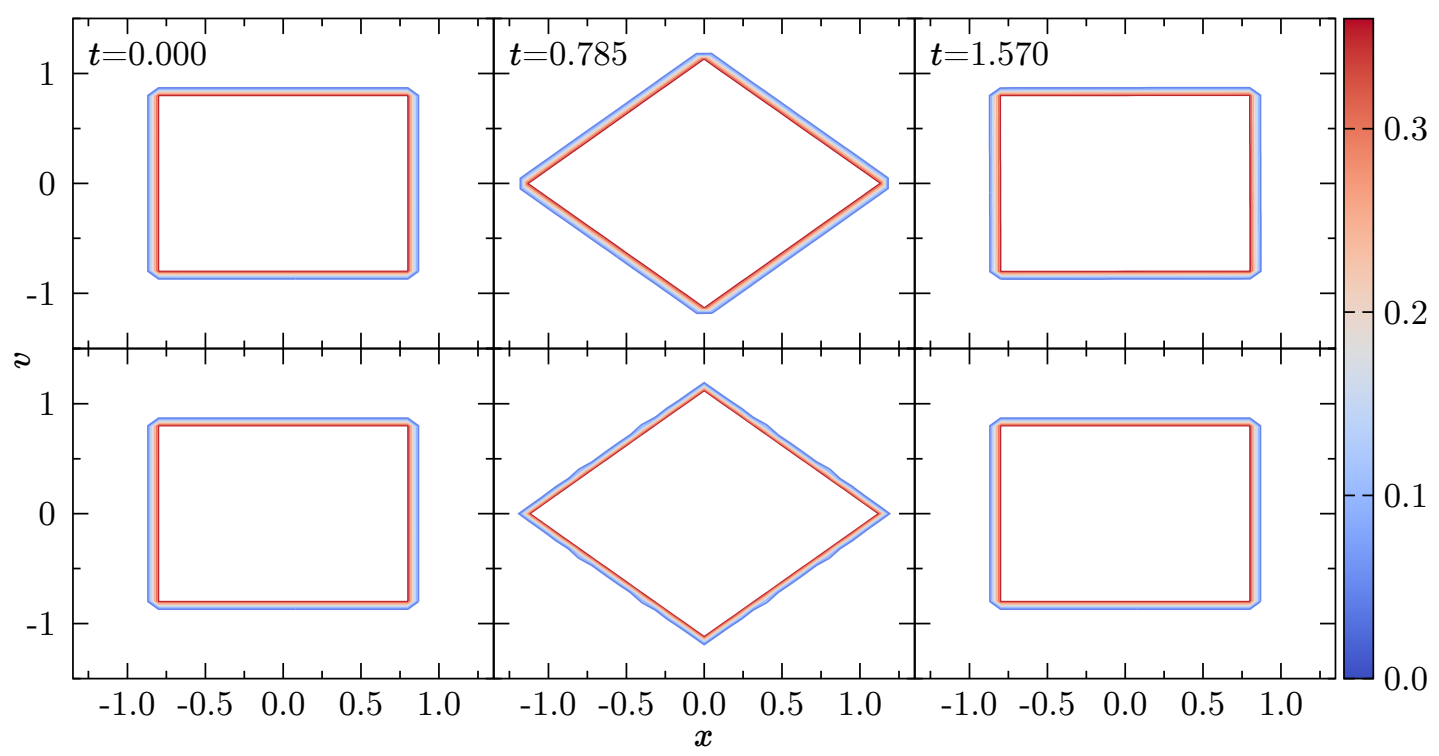

Figure 2.21: Evolution of $f(x, v x t)$ (top row) and its interpolation (bottom row) for an histogram initial condition and an external acceleration of $-x$.

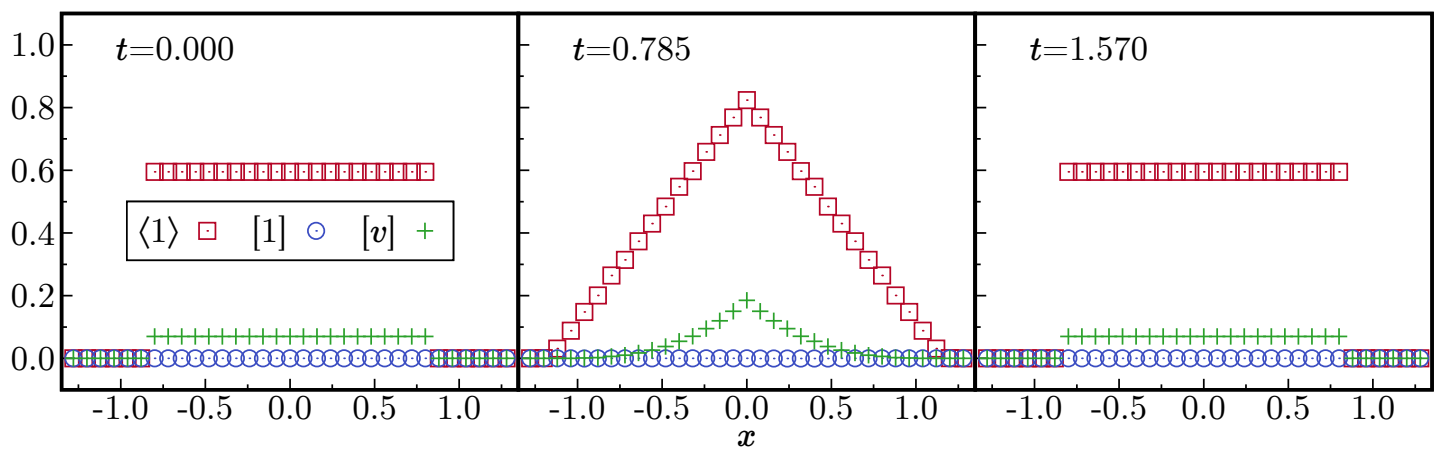

Figure 2.22: Norm and fluxes of order 0 and 1 of the interpolated function from figure 2.21.

certain period of time.

Hence, norm and fluxes of $f$ also evolve periodically, as seen in figure 2.22. At $t=$ $0.785 \simeq \pi / 4$ a perfect triangle, as analytically computed, of $\langle 1\rangle$ with zero [1] is obtained. The stability of the solution indicates that the PIM and the interpolation method are able to reproduce situations where classical numerical methods may introduce nonphysical effects.

\subsubsection{Numerical hindrances}

The main inconvenience to solve convective equations is the displacement of the mesh and the interpolation process that can perturb the simulations, specially when abrupt solutions appear. The absence of diffusive effects means that there is no need to have a minimum of points to properly represent the Gaussian-like propagator, yet the mesh 
motion can produce inaccuracies in the solution. To analyse this, in view of figure 2.17, where all points move at a constant rate $D_{q} \tau$ each time step and the initial mesh goes from -10 to 10 units. This means that after six time units it covers the space from -4 to 16 , as seen in figure 2.23. This can be a handicap, for example, if the integral quantities of $f$ are calculated, because values, specially in the tails of $f$ may be lost as the mesh adapts to the convective force $D_{q}$. This issue could be minimised applying some constrains to the mesh motion, however, the numerical and analytical consequences of these corrections must be deeply studied before applying them.

Another important topic related to non-diffusive equations is the numerical representation of Dirac delta and Heaviside functions. The Dirac delta is necessary to compute the propagator, meanwhile Heaviside function appears in its time-integral, which is necessary to include non-homogeneous terms. For these problems, the following numerical implementations of these functions are assumed: for the Dirac delta

$$
\delta_{i}\left(q_{i}\right)= \begin{cases}1 & \text { if }\left|q_{i}\right|<\epsilon \\ 0 & \text { elsewhere }\end{cases}
$$

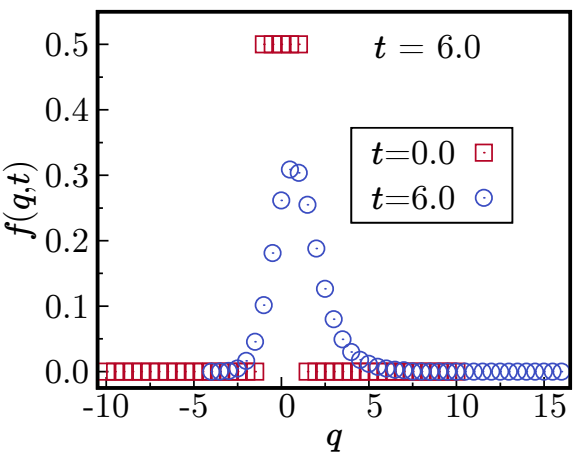

Figure 2.23: Initial and final mesh for non-homogeneous convective $1 D$ problem.

and for the Heaviside function

$$
H_{i}\left(q_{i}\right)=\left\{\begin{array}{ll}
1 & \text { if } q_{i}>\epsilon \\
0 & \text { elsewhere }
\end{array} ;\right.
$$

where $\epsilon$ is a small value (usually in the order of $10^{-14}$ ) that ensures that the small numerical errors that occur in the calculation of $q_{i}$ due to machine precision do not distort the numerical problem. Two things must be stressed here. First, the numerical delta is not divided by the space parameter $\Delta q$, which means the numerical advancing scheme becomes

$$
f_{i}^{n+1}=\sum_{i^{\prime}} P_{\tau_{i i^{\prime}}} f_{i^{\prime}}^{n}
$$

to ensure that the norm of the propagator is one. This is done to avoid numerical errors due to the division/multiplication of the same numerical value. Secondly, the Heaviside function is not taken as 0.5 for $q_{i}=0$ because, numerically, a value of the argument equal zero is almost impossible to appear, and providing a value of 0.5 for any other argument different from zero (even for a small value) produces invalid results in our cases. Nevertheless, other numerical implementations of these functions should be analysed to improve the purely convective solutions. 


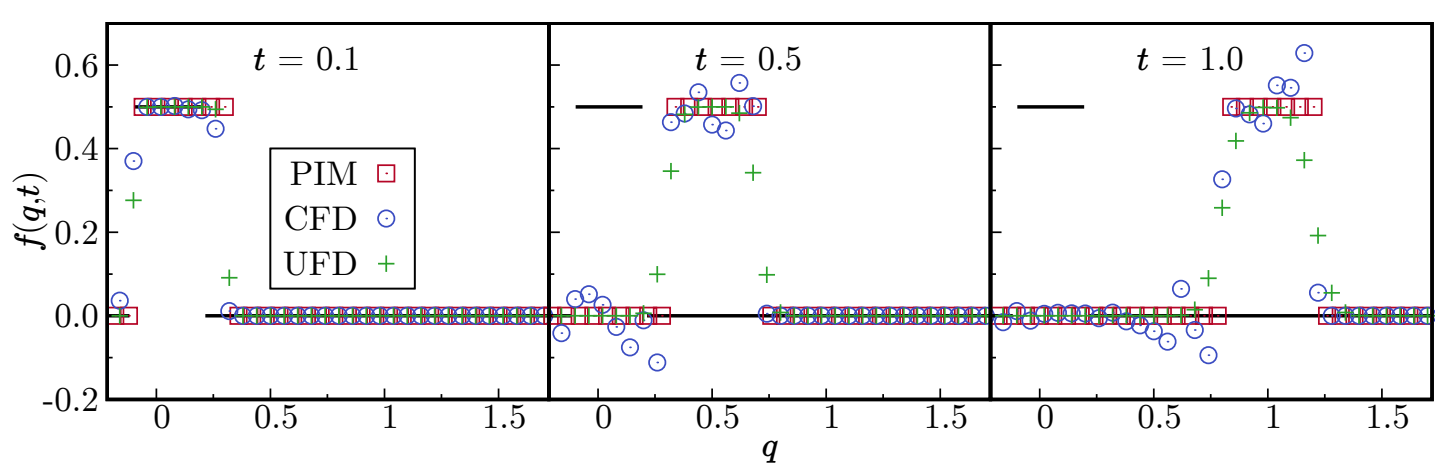

Figure 2.24: Evolution of an histogram-like initial condition solved by the PIM, central CFD and upwind UFD finite difference schemes. Initial condition is represented as a black solid line.

\subsubsection{Comparison with Finite Difference Methods and computational cost}

A basic analysis to understand the main conceptual difference between the PIM and classical FDM to solve pure-convective problems can be performed for the simple constant convective force case, solving

$$
\frac{\partial f}{\partial t}+\frac{\partial}{\partial q} D_{q} f=0
$$

for $D_{q}=1$. Two finite difference schemes are commonly employed here: upwind and central discretisation methods, establishing.

$$
f_{i}^{n+1}=f_{i}^{n}+\tau \frac{f_{i}^{n}-f_{i-1}^{n}}{\Delta q},
$$

for the upwind scheme, and

$$
f_{i}^{n+1}=f_{i}^{n}+\tau \frac{f_{i+1}^{n}-f_{i-1}^{n}}{2 \Delta q}
$$

for the central scheme; in usual notation. Starting from the drawer function, as

$$
f(q, 0)=f_{0}(q)= \begin{cases}0.5 & \text { if }-0.1<q<0.2 \\ 0 & \text { elsewhere }\end{cases}
$$

the problem should evolve towards increasing $q$ values, keeping the initial shape since the solution in time is trivially $f(q, t)=f_{0}\left(q-D_{q} t\right)$. The results solving this simple problem with the two finite differences methods and the PIM are presented in figure 2.24. The PIM solution shape coincide with the initial one and only a convective motion appears. On the other hand, finite difference solutions follow the convective evolution but their shape is highly disrupted. If the central discretisation scheme is used, oscillations appear in almost all the solution, leading to negative or higher values with respect to the initial condition. For the upwind scheme, a numerical viscosity arises, which changes the abrupt initial condition into a Gaussian-like function, losing 


\begin{tabular}{lll}
\hline Method & \multicolumn{2}{c}{ Time } \\
\hline \multirow{2}{*}{ Central } & Average time per iteration (ms) & 0.0178 \\
& Total time (s) & 211.52 \\
\multirow{2}{*}{ Upwind } & Average time per iteration (ms) & 0.0174 \\
& Total time (s) & 207.56 \\
\multirow{2}{*}{ PIM } & Average time per iteration (ms) & 22.413 \\
& Total time (s) & 0.2242 \\
\hline
\end{tabular}

Table 2.3: Computational times per iteration and total time for each method employed to solve equation (2.66) with $\Delta q=0.004$. Initialisation times are neglected. These simulations where executed in the same machine from table 2.1.

the essence of the pure-convective problem. In this particular example, all three cases are solved with $\Delta q=4 \cdot 10^{-3}$ but different time steps: 0.1 for the PIM and $10^{-7}$ for the FDM. This small time step, required to reduce the numerical errors, derives in a large number of iterations. Hence, the discretisation scheme affects the numerical solution in such a way that it does not reproduce the analytical evolution, whereas the PIM evolves as the analytical solution without introducing numerical instabilities or non-physical effects.

Respecting the computational cost of 2.24, table 2.3, due to the large $\tau$ that PIM can use, the number of iterations required to solve the same time can be extremely decreased, reducing the total computation time. However, as it happened in the diffusive case, the time per iteration is larger in the PIM case.

Besides the cost of computing and saving the full propagator in memory, which in the pure convective case is reduced because the only possible values for the propagator are 1 or 0 , the resolution of purely convective equations with the PIM requires the movement of the grid to represent the local convective effect and, in some cases, the calculation of an interpolated function to compute the distribution moments and fluxes. In figure 2.25 , the average time of the test-case from figure 2.19 are pictured (being New grid the calcula-

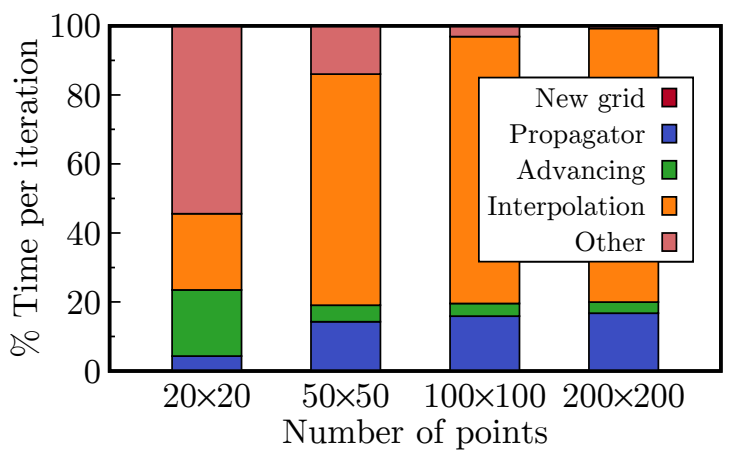

Figure 2.25: Percentage of the iteration time employed by each task of a $1 D 1 \mathrm{~V}$ purely convective simulation for different grid sizes. tion of the new points, Propagator the computation of $P_{\tau}$, Advancing the integration process to advance the solution, Interpolation the computation of the solution in a fixed mesh and Others accounting for initialisations, data output and other small operations) are presented as a percentage of the iteration time. The number of points defines the 
requisites for each task, but it is clear that the interpolation process consumes almost all the time of the iteration. In order to reduce the time per iteration, the presented interpolation method in appendix B.5 should be improved. Due to the similarity of this fixed mesh with the one employed in PIC methods to obtain the macroscopic quantities, some interpolation routines could be adapted to this case. Furthermore, the calculation of a new mesh does not increase the computational time, which means that its simple operation does not add extra computational cost.

\subsection{Application to Ordinary Differential Equations (ODE)}

In many practical problems, the partial differential equations can be reduced to ODEs, as in the calculation of the electric field in one-dimensional problems where the Poisson's equation is employed to obtain the electric potential in the electrostatic approximation. Classical resolution of these problems require discretisation and matrix inversion, which can result in large computational efforts for accurate results.

The basic procedure to solve these problems on the basis of integral methods is found in reference [47, Section 0.8.3]. Here, a procedure from the point of view of the Propagator Integral Method is presented.

The general ODE problem is usually described as

$$
\mathfrak{L}_{q} f=s(f, q),
$$

with additional boundary conditions in the domain edge, where $\mathfrak{L}_{q}$ represents any lineal combination of derivatives of $f$ in the $q$ direction. Even when this problem does not directly depend on time, a numerical iterative process can be introduced to solve it, which intermediate states have no physical meaning and its sole propose is to find a converged state. This may lead to situations where a fast and accurate calculation at each step can reduce significantly the computation time, producing a better global solution. In other words, (2.70) is the steady solution of $\partial f / \partial \tau+\mathfrak{L}_{q} f=s$ for the parameter $\tau \rightarrow \infty$

In this section, the PIM is expanded to deal with this type of equations in a onedimensional planar space as the cases shown below. Even when this approach is very common, it is important for this work to extend the PIM methodology to these problems, to avoid the resolution by classical difference methods and unify the numerical procedure used to solve all equations appearing in this dissertation. Here we focus in the resolution of the Poisson's equation, but procedure can be easily extended to deal with other ODE types. 


\begin{tabular}{llll}
\hline Type of BC & Value at $q_{b}$ & Condition for P & Function $Q$ \\
\hline Dirichlet & $f\left(q_{b}\right)=g(q, f)$ & $P\left(q_{b}, q^{\prime}\right)=0$ & $Q\left(q, q^{\prime}\right)=-D_{q q}^{\prime} \frac{\partial P}{\partial q^{\prime}}$ \\
Neumann & $\left.\frac{\partial f}{\partial q}\right|_{q_{b}}=g(q, f)$ & $\left.\frac{\partial P}{\partial q}\right|_{q_{b}}=0$ & $Q\left(q, q^{\prime}\right)=P\left(q, q^{\prime}\right)$ \\
\hline
\end{tabular}

Table 2.4: Boundary conditions for ODE problem, auxiliary conditions for $P$ calculation, and shape of $Q$. Point $q_{b}$ identifies the boundary.

\subsubsection{One-dimensional Poisson's equation}

Here, we explore the possibility of using integral schemes to solve the Poisson's equation, which appear in many models and system of equations. An important application in plasma modelisation is to compute the electrical potential when a charge separation occurs. The form of Poisson's equation in a one-dimensional planar space is

$$
\frac{\partial^{2} D_{q q} f}{\partial q^{2}}=s(q, f)
$$

where $D_{q q}=D_{q q}(q, f)$ is the diffusion parameter and boundary conditions are applied at points $q_{0}$ and $q_{f}$. As done previously, a propagator, which propagates pulses in $q$ not in time as in previous problems, is found solving the auxiliary equation

$$
\frac{\partial^{2} D_{q q} P}{\partial q^{2}}=\delta\left(q-q^{\prime}\right)
$$

with the proper boundary conditions from table 2.4. The well-known general integral scheme for these problems is $[46,48]$

$$
f(q)=\int_{q_{0}}^{q_{f}} s^{\prime} P\left(q, q^{\prime}\right) \mathrm{d} q^{\prime}-g_{0} Q\left(q, q_{0}\right)+g_{f} Q\left(q, q_{f}\right),
$$

defined in $\left[q_{0}, q_{f}\right]$. The two boundary conditions $g_{0}$ and $g_{f}$ indicates the value of the function (or its derivative) at the extreme points $q_{0}$ and $q_{f}$ respectively. The form of $Q\left(q, q^{\prime}\right)$ is in in table 2.4 for each case. Function $Q$ transfers the value at the boundaries to all the domain, as it happened in the one-dimensional drift-diffusion case. In addition, the propagator is modified as it has to fulfil a homogeneous boundary condition.

Let us try now a simple example, the double Dirichlet boundary conditions, i.e., the value of $f$ is imposed at $q_{0}$ and $q_{f}$. The propagator that fulfils (2.72) and the auxiliary boundary conditions is

$$
\begin{aligned}
P\left(q, q^{\prime}\right)=\left(q-q^{\prime}\right) H\left(q-q^{\prime}\right)+\frac{1}{D_{q q} L}[ & \left(q-q_{f}\right)\left(q_{0}-q^{\prime}\right) H\left(q_{0}-q^{\prime}\right) \\
& \left.+\left(q-q_{0}\right)\left(q_{f}-q^{\prime}\right) H\left(q_{f}-q^{\prime}\right)\right],
\end{aligned}
$$




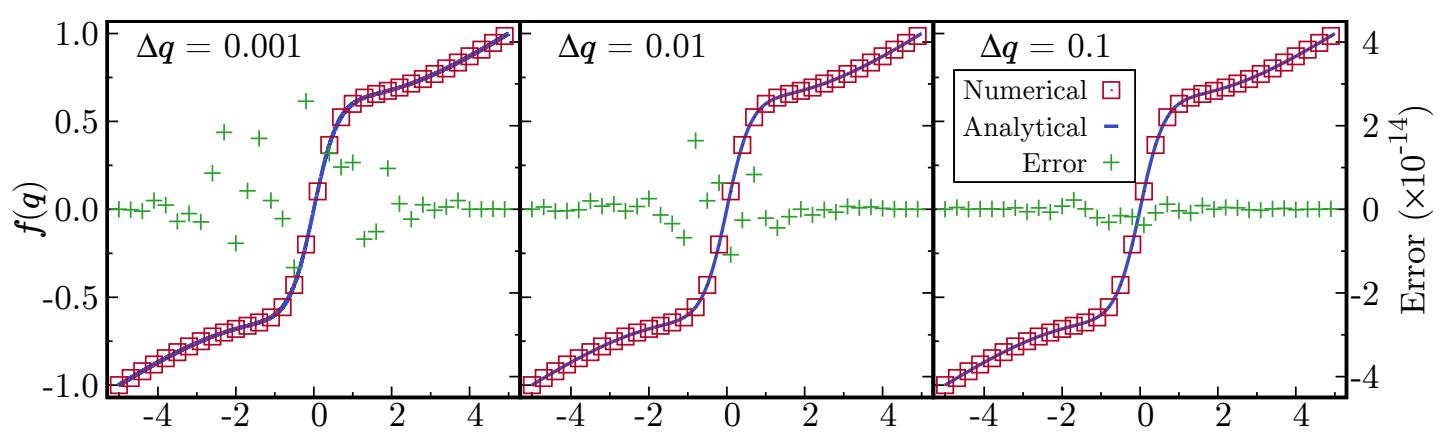

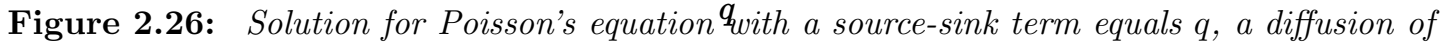
the form $1+q^{2}$ and Dirichlet boundaries equal \pm 1 at $q= \pm 5$. The numerical and the analytical solutions on left axis. Difference of both solution is presented in the right axis.

where $L=q_{f}-q_{0}>0$ and $H$ is the Heaviside function. Following table 2.4, the function $Q$ becomes

$$
\begin{aligned}
Q\left(q, q^{\prime}\right)=\frac{D_{q q}^{\prime}}{D_{q q}} H\left(q-q^{\prime}\right)+\frac{D_{q q}^{\prime}}{D_{q q} L}[ & \left(q-q^{\prime}\right)\left(q_{f}-q_{0}\right) \delta\left(q-q^{\prime}\right) \\
& +\left(q-q_{f}\right)\left(q_{0}-q^{\prime}\right) \delta\left(q_{0}-q^{\prime}\right) \\
& -\left(q-q_{0}\right)\left(q_{f}-q^{\prime}\right) \delta\left(q_{f}-q^{\prime}\right) \\
& +\left(q-q_{f}\right) H\left(q_{0}-q^{\prime}\right) \\
& \left.+\left(q_{0}-q\right) H\left(q_{f}-q^{\prime}\right)\right] .
\end{aligned}
$$

It is important to remark that to obtain this propagator, not short-time approximation of the diffusive coefficient is required (the parameter $D_{q q}$ is evaluated in source variables, not in primed ones) since it does not depend on a time $t$, which improves the accuracy of the numerical computations. Obviously, an approximation is still required to transfer this analytical function into a numerical domain. From (2.73) it is deduced that only the values of $Q$ at $q^{\prime}=q_{0}$ and $q^{\prime}=q_{f}$ are required, which simplifies the numerical problem in terms of memory and computation resources required.

As a first example, a non-homogeneous term of value $s=q$, a non-constant diffusion as $D=1+q^{2}$ and boundary conditions $f(-5)=-1$ and $f(5)=1$ is solved. The results for $\Delta q=0.001,0.01$ and 0.1 are presented in figure 2.26. In this example, numerical solutions agree almost perfectly with the analytical one, which can be easily obtained, and only small errors due to the translation from analytical to numerical algorithm appear.

A special application of the Poisson's equation in a semi-open domain valid for the problem of the interaction of a plasma and a wall is studied. The finding of this potential is key to describe the evolution of the system as its derivative correspond to the electric field. The Poisson's equation as $q_{0}=0$ and $q_{f} \rightarrow \infty$, with a Dirichlet condition at 0 , which represents the potential of a metallic wall, and zero derivative at $\infty$, which indicates that the quasi-neutrality of the plasma is recovered far from the wall. The calculation of the new propagator follows (2.72) but with the homogeneous boundary conditions required by the problem. One propagator valid to solve this 


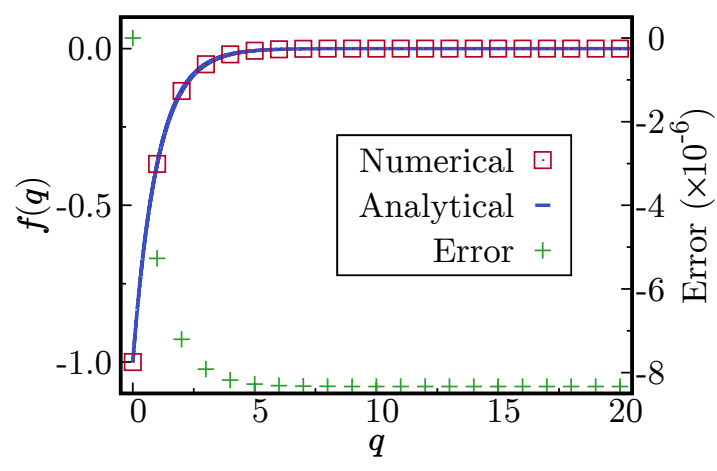

(a) Solution

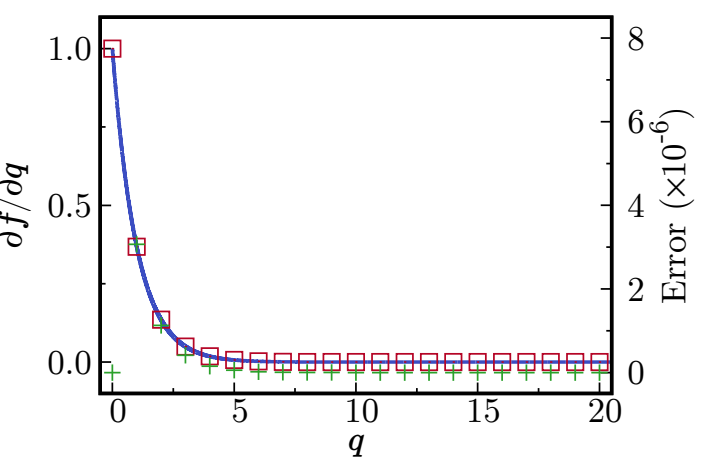

(b) Solution's derivative

Figure 2.27: Solution for the Poisson's equation and its derivative in the semi-open domain. The incorporation of a boundary condition at $\infty$ perturb the solution and increases the order of magnitude of the error.

problem assuming $D_{q q}=1$ is

$$
P\left(q, q^{\prime}\right)=\left(q-q^{\prime}\right) H\left(q-q^{\prime}\right)-q,
$$

where $Q$ becomes

$$
Q\left(q, q^{\prime}\right)=H\left(q-q^{\prime}\right)
$$

Again, we are only interested in the value of $Q(q, 0)$, because the boundary condition at infinity is included inside the structure of the propagator and the $Q$ function. Hence no extra information is required to account for the effect of $+\infty$. For this problem, a non-homogeneous term that vanish as $q \rightarrow \infty$ is selected. This is a requirement to avoid inconsistencies because we have imposed a zero derivative of the function at $q \rightarrow \infty$. The particular non-homogeneous term taken is $s=-\exp [-q]$ and $f(0)=-1$. The analytical solution (and its derivative) for this problem are

$$
\begin{aligned}
f(q) & =1-\exp (-q)+f_{0}, \\
f^{\prime}(q) & =\exp (-q)
\end{aligned}
$$

For the numerical problem, the derivative is computed by a simple central scheme for the inner points and upwind forward and backwards respectively for the first and last points. It can be observed in figure 2.27 that both, the function and its derivative agree with the analytical solution having a maximum error of the order $10^{-6}$. The inclusion of a boundary condition at infinity increases the error of the solution due to the finiteness of the numerical grid. However, the analytical kernel of $P$ allows a proper representation of boundary conditions involving in practice $\pm \infty$ in a finite numerical domain, as the boundary is not imposed at the end of the numerical domain.

\subsubsection{Numerical hindrances}

As it happened with the resolution of time depending pure convective equations, the numerical representation of Dirac delta and the Heaviside function may present an issue. 


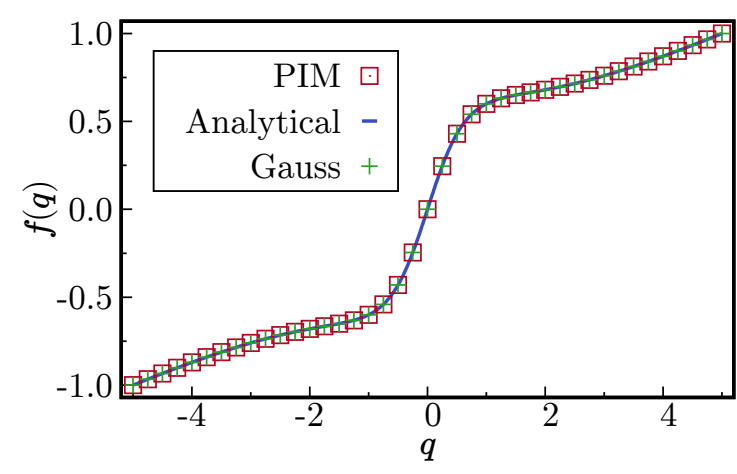

(a) Solutions

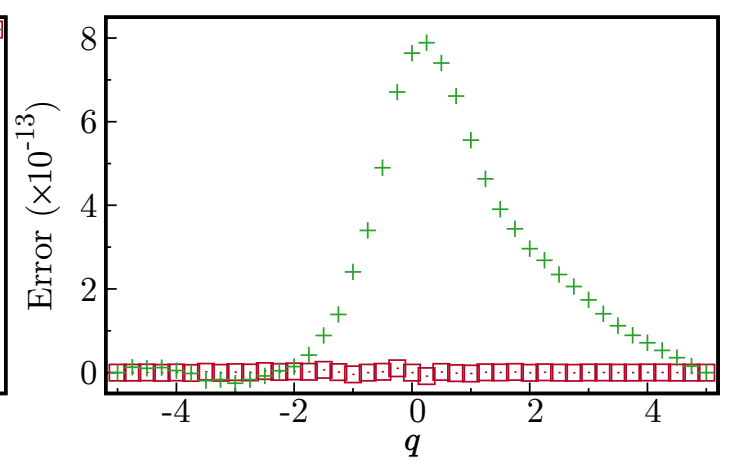

(b) Solution errors

Figure 2.28: Resolution of Poisson's equation with a non-homogeneous term and a diffusion coefficient equal $q$ and $1+q^{2}$ respectively. Solution obtained by the PIM is presented with red squares has an error respect to the analytical function in the order of $10^{-15}$. Both solutions are solved with a $\Delta q=0.001$.

To solve these problems, same techniques as those employed in the pure convective problems are employed.

The integral numerical scheme

$$
f_{i}=\sum_{i^{\prime}=0}^{q_{\max }} s_{i^{\prime}} P_{i i^{\prime}} \Delta q-q_{0} Q_{i 0}+q_{f} Q_{i f},
$$

where 0 and $f$ indicate the first and last grid points, respectively, produces a solution valid to all the domain $q$, accounting for boundary conditions and non-homogeneous effects. As in previous problems, boundary conditions in FDM only affect the ending points, whereas the function $Q\left(q, q^{\prime}\right)$ acts over all the domain, acting as an infinite order scheme. Also, the absence of discretisation rules means that no minimum number of points is required to ensure the stability of the solution, unless increasing the accuracy is desired.

\subsubsection{Comparison with Finite Difference Methods and computational cost}

The resolution of ODEs by classic FDM usually requires matrix inversion, that can be solved directly or by iterative process or complex algorithm of high computational cost.

A comparison of PIM solution from figure 2.26 with the one obtained by a direct matrix inversion method for $\Delta q=0.001$ is used to compare the computational effort. In this kind of methods, the discretisation and boundary conditions that affect function $f$ are included in a $\left(q_{\max }+1\right) \times\left(q_{\max }+1\right)$ matrix to be inverted, which for the Poisson's 


\begin{tabular}{lcc}
\hline Method & \multicolumn{2}{c}{ Time } \\
\hline PIM & Total time (s) & 0.0104 \\
Gauss & Total time (s) & 0.8169 \\
\hline
\end{tabular}

Table 2.5: Computational times for the PIM and direct matrix inversion method (Gauss) to solve Poisson's equation.

problem with Dirichlet boundary conditions appears as

$$
\frac{1}{\Delta q^{2}}\left[\begin{array}{ccccccc}
1 & 0 & 0 & 0 & 0 & \ldots & 0 \\
1 & -2 & 1 & 0 & 0 & \ldots & 0 \\
0 & 1 & -2 & 1 & 0 & \ldots & 0 \\
\vdots & & \ddots & \ddots & \ddots & & \vdots \\
0 & 0 & 0 & 0 & 0 & \ldots & 1
\end{array}\right] \cdot\left[\begin{array}{c}
D_{q q}\left(q_{0}\right) f_{q_{0}} \\
\vdots \\
D_{q q}\left(q_{i}\right) f_{q_{i}} \\
\vdots \\
D_{q q}\left(q_{f}\right) f_{q_{f}}
\end{array}\right]=\left[\begin{array}{c}
\frac{D_{0} f_{0}}{\Delta q^{2}} \\
s_{1} \\
\vdots \\
s_{i} \\
\vdots \\
s_{q_{f}-1} \\
\frac{D_{q_{f}} f_{q_{f}}}{\Delta q^{2}}
\end{array}\right]
$$

where the first and last rows include the effect of the boundary condition $\left(f_{q_{\max }}\right.$ and $f_{0}$ are given). The problem can be symbolised in vector form as $\mathfrak{B} \cdot \mathbf{D}_{q q} f=\mathbf{s}$, solved as $\mathbf{D}_{q q} f=\mathfrak{B}^{-1} \cdot \mathbf{s}$. Here, a direct Gauss method is used to $\mathfrak{B}$ inversion. In figure 2.28 both numerical solutions and the analytical function are compared. The error of the integral solution remains in the order of $10^{-15}$, while for the direct matrix solution the error is two order of magnitude higher. Moreover, this error increases close to $q=0$, where the slope of the solution increases, proving that the discretisation error depends on the slope value. In table 2.28, the times to solve this problem by both methods are written. It is important to notice that the Gauss method requires a large time to invert the matrix and that this could be improved by an iterative procedure. In these problems, no paralellisation has been set for any method.

\subsection{Conclusions}

In this chapter, the Propagator Integral Method (PIM) has been extended and applied to solve a large class of usual partial and ordinary differential equations. Some guidelines have been given to apply the method to different geometries, boundary conditions and equation types. In addition, a resume of the most general numerical difficulties that can be found using this method has been presented. This is completed with the listings presented in appendix B. These impediments include problems with boundary conditions and number of points for low diffusion values, which require a deeper analysis. Nevertheless, the method has been proven as an excellent tool to solve highly 
non-linear problems in a consistent way, providing solutions very close to the analytical ones.

Drift-diffusion equations, dealt in previous works, have been addressed in depth. Additionally, the method has been extended to solve purely convective problems and the one-dimensional Poisson's equation, both commonly appearing in plasma descriptions. A wide variety of cases resembling those found in typical plasma kinetic models have been solved.

The comparison with classical FDM has shown that big differences, specially for the pure-convective equations, where solutions obtained with the PIM have no numerical oscillations and the method does not introduce an extra viscosity. Solutions obtained with the PIM are impossible by commonly used approaches in FDM, as the method evolves the solution as if it is analytical for short-times.

The Propagator Integral Method opens new possibilities to solve complex non-linear problems beyond its first application to time evolving equations as the Fokker-Planck one. In the following chapters, this method is applied to solve kinetic problems in Plasma Physics, but it is important to stress that the equations dealt in this chapter appear vastly among different branches of mathematics, physics and engineering, and many fields could benefit from the advances performed in this chapter. 


\section{CHAPTER 3}

\section{AN OPERATIVE INTRODUCTION TO PLASMA PHYSICS}

A plasma is considered as a mixture of electrons, positive or negative ions and neutral particles that exhibit a collective behaviour. Due to this collective response, the electromagnetic field is used to control or confine the plasma. Moreover, microscopic processes among particles are very common to occur, producing exchange of energy among the species and modifying their dynamics.

Usually, plasmas are characterised attending to the electron density and temperature. A comprehensive classification is synthesised in figure 3.1, where different types of plasmas are organised based on these two properties. As it can be seen, plasmas vary in a large range of density and temperature which hinders the development of models suitable for large ranges of configurations, because some specific phenomena dominate each type of plasma, leading its evolution and giving rise to disparate

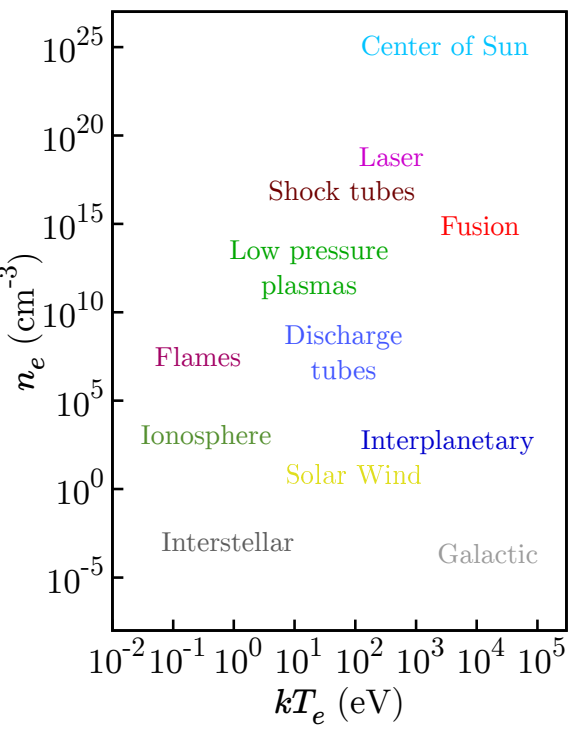

Figure 3.1: Classification of plasmas by electron density and temperature. Typical examples of some common plasmas are presented. scenarios. In these different regimes, a variety of process arises with higher or lower intensities, creating very different plasma dynamics. Hence, plasma models are mainly traced to a better representation of the relevant phenomena characterising each plasma type to obtain simplified models. This emerges from the necessity to allow the resolution of involved systems, unsolvable by other means. Nevertheless, some basic processes and analysis can be performed in order to 
describe plasmas from a quite general perspective, allowing a better understanding of the specific processes.

In this chapter, an introduction to general description of plasmas is given to establish the foundation of terminology employed in the forthcoming chapters. To this end, a general view of kinetic theory is presented. Then, a basic description of fluid equations is given. Although no fluids models are solved through this dissertation, it is important to analyse the behaviour of macroscopic moments and fluxes obtained from the distribution function to improve the understanding of results commonly outputted from kinetic models. In Kinetic models, the direct analysis of the distribution function obtained may not provide enough information to fully understand the plasma dynamics since its variations may be very small. Therefore, the analysis of integrated quantities becomes a required procedure to extract useful information from kinetic models. Finally, the Vlasov-Poisson system of equations, commonly used to describe non-collisional plasmas, is studied. Some numerical cases, including the case of two species non-collisional plasma (ions and electrons) evolving under a self-consistent electric field, are solved in a $1 \mathrm{D} 1 \mathrm{~V}$ planar geometry using the PIM for non-diffusive equations presented in section 2.3.2.

\subsection{Kinetic theory in plasmas}

The kinetic theory is a useful tool to analyse the evolution of plasmas and gases due to its capability to describe, from a collective point of view, the microscopic interactions among particles. However, the resulting systems of equations derived are usually hard to solve, even with robust numerical methods, due to the high non-linearities and strong coupling among them. Moreover, the high-dimensional phase-space $\mathbf{r}-\mathbf{v}$ involved in these problems results in numerical models that require too much computational cost to solve, unavailable in most cases.

The main goal of this theory is to describe the plasma by using each species distribution function, which represents the amount of particles that exists in a differential volume $\mathrm{d} \mathbf{r} \mathrm{d} \mathbf{v}$ at position $\mathbf{r}$ and velocity $\mathbf{v}$ at time $t$. It plays the role of a density in phase space and it is denoted as $f_{\gamma}(\mathbf{r}, \mathbf{v}, t)$ where $\gamma$ indicates the kind of particle, i.e., the species. Moreover, $f_{\gamma}$ is positively defined and its tails usually vanishing for $|\mathbf{v}| \rightarrow \infty$. The evolution of the distribution function for a generic species $\gamma$ is represented by the well-known Boltzmann equation [78, Section 5.5]

$$
\frac{\partial f_{\gamma}}{\partial t}+\mathbf{v} \cdot \nabla_{\mathbf{r}} f_{\gamma}+\frac{\mathbf{F}}{m_{\gamma}} \cdot \nabla_{\mathbf{v}} f_{\gamma}=\sum_{\gamma^{\prime}}\left(\frac{\partial f_{\gamma}}{\partial t}\right)_{\gamma \gamma^{\prime}}+s_{\gamma},
$$

where $m_{\gamma}$ is the particle mass, $\mathbf{F}$ represents an external force, usually the Lorentzian one $\mathbf{F}=q_{\gamma}(\mathbf{E}+\mathbf{v} \times \mathbf{B})$, where $\mathbf{E}$ and $\mathbf{B}$ are the electric and magnetic fields, respectively, and $q_{\gamma}$ is the particle charge. The time rate of change contribution $\left(\partial f_{\gamma} / \partial t\right)_{\gamma \gamma^{\prime}}$ is related to the interaction of particles of kind $\gamma$ with other species $\gamma^{\prime}$ and $s_{\gamma}$ is a 
non-homogeneous term, that usually contains charge-exchange phenomena, ionizationrecombination processes, and sink-sources of particles. A rigorous derivation of this equation can be found in the literature $[56,78,79]$. To our aims here, it is enough to understand (3.1) as a continuity equation for density $f_{\gamma}$ in the phase space.

Nevertheless, a consideration must be made about the Boltzmann equation. Traditionally, elastic collision terms in (3.1) are represented by the Boltzmann collisional operator

$$
\left(\frac{\partial f_{\gamma}}{\partial t}\right)_{\gamma \gamma^{\prime}}=\int g \sigma(g, \Omega)\left[f_{\gamma}\left(\mathbf{r}, \mathbf{v}^{\prime}, t\right) f_{\gamma^{\prime}}\left(\mathbf{r}, \mathbf{v}_{*}^{\prime}, t\right)-f_{\gamma}(\mathbf{r}, \mathbf{v}, t) f_{\gamma^{\prime}}\left(\mathbf{r}, \mathbf{v}_{*}, t\right)\right] \mathrm{d} \Omega \mathrm{d} \mathbf{v}_{*},
$$

where $g=\left|\mathbf{v}-\mathbf{v}_{*}\right|=\left|\mathbf{v}^{\prime}-\mathbf{v}_{*}^{\prime}\right|$ is the module of the relative velocity, $\sigma(g, \Omega)$ is the differential cross-section for the elastic interaction between $\gamma$ and $\gamma^{\prime}$, and $\Omega$ is the scattering angle. Here the auxiliary velocities $v_{*}^{\prime}$ and $v^{\prime}$ are the after collision velocities, expressed as

$$
\begin{aligned}
\mathbf{v}^{\prime} & =\frac{\mathbf{v}+\mathbf{v}_{*}}{2}+\frac{\left|\mathbf{v}-\mathbf{v}_{*}\right|}{2} \sigma \\
\mathbf{v}_{*}^{\prime} & =\frac{\mathbf{v}+\mathbf{v}_{*}}{2}-\frac{\left|\mathbf{v}-\mathbf{v}_{*}\right|}{2} \sigma .
\end{aligned}
$$

However, collisional terms in (3.1) should not be limited to this specific description. Approximations as the BGK term [80,81], or integro-differential operators like the Fokker-Planck-Landau, which can be obtained independently of the Boltzmann collision operator as presented by B. A Trubnikov [82], can be used without loosing the sense of balance equation that (3.2) provides. The collection of collision operators employed in this work is investigated in chapter 4 , where analytical and numerical studies are carried out.

One of the major advantages of kinetic theory is that allows the calculation of macroscopic measurable variables and fluxes by directly integrating the distribution function over the velocity space domain $D_{\mathbf{v}}$. This means $f_{\gamma}(\mathbf{r}, \mathbf{v}, t)$ has a macroscopic variable $\left\langle\chi_{\gamma}\right\rangle$, where $\chi_{\gamma}$ is any function of independent variables as

$$
\left\langle\chi_{\gamma}\right\rangle=\frac{1}{n_{\gamma}} \int_{D_{\mathbf{v}}} \chi_{\gamma}(\mathbf{r}, \mathbf{v}, t) f_{\gamma} \mathrm{d} \mathbf{v}
$$

where $n_{\gamma}$ is the number of particles per unit of volume and the zero order moment of the distribution function, expressed as

$$
n_{\gamma}=\int_{D_{\mathbf{v}}} f_{\gamma} \mathrm{d} \mathbf{v}
$$

also known as norm. For example, if $\chi_{\gamma}=\mathbf{v}$, the macroscopic velocity $\mathbf{u}_{\gamma}$, the fluid velocity or just velocity, is obtained. The temperature from the distribution function, $k T_{\gamma} / 2 m_{\gamma}$, where $k$ is the Boltzmann constant, is obtained setting $\chi_{\gamma}=\frac{1}{2}\left|\mathbf{v}-\mathbf{u}_{\gamma}\right|^{2}$, and the total energy per unit of mass and density is calculated by the addition of internal and kinetic energy per unit of mass, as $E_{\gamma}=k T_{\gamma} / 2 m_{\gamma}+\frac{1}{2} u_{\gamma}^{2}$, or by calculating (3.5) 
with $\chi_{\gamma}=\frac{1}{2} v^{2}$ where $u_{\gamma}$ and $v$ are the modules of the vectors $\mathbf{u}_{\gamma}$ and $\mathbf{v}$, respectively. It is important to remark that these properties may depend on the position and time, as the distribution function changes during the evolution of the problem following equation (3.1).

Some other important macroscopic quantities obtained from the distribution function are the fluxes $\left[\psi_{\gamma}\right]$. These fluxes express how macroscopic quantities are transported through the problem. They are obtained directly from the distribution function as

$$
\left[\psi_{\gamma}\right]=\int_{D_{\mathbf{v}}} \mathbf{v} \psi_{\gamma}(\mathbf{r}, \mathbf{v}, t) f_{\gamma} \mathrm{d} \mathbf{v}
$$

For example, if $\psi=m_{\gamma}$, the resulting flux is the mass flux $\mathbf{j}_{\gamma}$, also equal to $\rho_{\gamma} \mathbf{u}_{\gamma}$, which provides information on how mass density $\rho_{\gamma}=m_{\gamma} n_{\gamma}$ evolves. Moreover, in plasmas is typical to analyse the electrical current density, defined as $\mathbf{J}_{\gamma}=\left[q_{\gamma}\right]=$ $q_{\gamma} n_{\gamma} \mathbf{u}_{\gamma}$, which is a parameter related to the electrical current measured in laboratories by means of, for example, electrical probes [28,83-85]. In addition, total heat flux can be computed by $\mathbf{Q}_{\gamma}=\left[v^{2}\right] / 2 n_{\gamma}$. These moments and fluxes are of huge importance for two reasons: the calculation of transport coefficients, where they respond to perturbations in the electromagnetic field or spatial gradients; and to obtain an observable physically meaningful result from the distribution function, which is usually arduous to analyse by itself.

It is well-known that when (3.1) is solved with the Boltzmann collision operator in an absence of external forces and other effects as inelastic interactions with other species or boundary conditions, a Maxwellian distribution is obtained as a steady solution, described as

$$
f_{\gamma}(\mathbf{v})=n_{\gamma}\left(\frac{m_{\gamma}}{2 \pi k T_{\gamma}}\right)^{\frac{3}{2}} e^{\frac{-m_{\gamma}\left|\mathbf{v}-\mathbf{u}_{\gamma}\right|^{2}}{2 k T_{\gamma}}}
$$

where $n_{\gamma}, \mathbf{u}_{\gamma}$ and $T_{\gamma}$ are the species steady density, mean velocity and temperature, respectively. This solution fulfils the H-theorem [78] as it is the distribution function of maximum entropy that can be obtained. Nevertheless, it is important to remember that, in plasmas, it is common to have situations where, due to phenomena as boundary conditions or interactions with other species, Maxwellian distributions are not reached and other distribution function shapes may arise. For example, in solar wind $[86,87]$ Lorentzian or $\kappa$-like distributions commonly appear, characterised by higher velocity tails than the Maxwellian. Furthermore, when plasma-wall interaction is analysed, the interplay of emission and collection of charged particles may disrupt the ideal Maxwellian equilibrium [88, 89].

Usually, Maxwell equations must be coupled with (3.1) to account for the self-consistent electromagnetic field created by variations of charge and current densities. These equa- 
tions read

$$
\begin{aligned}
\nabla_{\mathbf{r}} \cdot \mathbf{E} & =\frac{\rho_{c}}{\epsilon_{0}} \\
\nabla_{\mathbf{r}} \cdot \mathbf{B} & =0 \\
\nabla_{\mathbf{r}} \times \mathbf{E} & =-\frac{\partial \mathbf{B}}{\partial t} \\
\nabla_{\mathbf{r}} \times \mathbf{B} & =\mu_{0}\left(\mathbf{J}+\epsilon_{0} \frac{\partial \mathbf{E}}{\partial t}\right),
\end{aligned}
$$

where $\rho_{c}=\sum q_{\gamma} n_{\gamma}$ is the charge density, $\mathbf{J}=\sum \mathbf{J}_{\gamma}$ is the total electrical current density and $\epsilon_{0}$ and $\mu_{0}$ are the permittivity and permeability of free space, respectively. These equations provide the Lorentz force $\mathbf{F}=q_{\gamma}(\mathbf{E}+\mathbf{v} \times \mathbf{B})$ that appears in equation (3.1) generating the final system of coupled equations. In addition, appropriate boundary and initial conditions for $\mathbf{E}$ and $\mathbf{B}$ must be included, as well as other forces, of different nature, not considered here.

\subsection{Fluid theory}

Although no fluid models are solved in this dissertation, it is important to have a basic understanding of how macroscopic quantities behave and evolve. Basic equations to describe plasmas as fluids, founded on time and space evolution of macroscopic quantities are mainly affected by boundary conditions, gradients and fluxes and the electromagnetic field solved through Maxwell equations. The resulting motion equations also resemble the form of a FP equation and the PIM may also be applicable, although this is out of this thesis scope. As was explained above, kinetic theory allows the calculation of macroscopic fluxes and of the transport coefficients employed to close the set of fluid equations hierarchy. Moreover, any kinetic description of a system should produce a macroscopic evolution that agrees with fluid equations.

Basic equations to describe plasma as a fluid can be obtained from kinetic theory $[78,90]$ from the general Boltzmann equation (3.1). Thus, the mass density $\rho_{\gamma}$ obeys

$$
\begin{aligned}
\frac{\partial}{\partial t} \int m_{\gamma} f_{\gamma} \mathrm{d} \mathbf{v}+\int m_{\gamma} \mathbf{v} \cdot \nabla_{\mathbf{r}} f_{\gamma} \mathrm{d} \mathbf{v}+ & \int \mathbf{F} \cdot \nabla_{\mathbf{v}} f_{\gamma} \mathrm{d} \mathbf{v}= \\
& \int m_{\gamma} \sum_{\gamma^{\prime}}\left(\frac{\partial f_{\gamma}}{\partial t}\right)_{\gamma \gamma^{\prime}} \mathrm{d} \mathbf{v}+\int m_{\gamma} s_{\gamma} \mathrm{d} \mathbf{v},
\end{aligned}
$$

which can be written as

$$
\frac{\partial \rho_{\gamma}}{\partial t}+\nabla_{\mathbf{r}} \cdot \rho_{\gamma} \mathbf{u}_{\gamma}=\int m_{\gamma} s_{\gamma} \mathrm{d} \mathbf{v}
$$

if no exchange of particles is produced by elastic collisional effects, which is a continuity equation for the density. It is easy to check that $\rho_{\gamma}$ only changes due to a non-constant flux of particles or by the source-sink terms. 
The procedure can be extended to study the conservation of higher order moments, as for example, the evolution of mass flux $\left[m_{\gamma}\right]$ from

$$
\begin{array}{r}
\frac{\partial}{\partial t} \int \mathbf{v} m_{\gamma} f_{\gamma} \mathrm{d} \mathbf{v}+\nabla_{\mathbf{r}} \cdot \int m_{\gamma} f_{\gamma} \mathbf{v} \mathbf{v} \cdot \mathrm{d} \mathbf{v}+\int \mathbf{v} \mathbf{F} \cdot \nabla_{\mathbf{v}} f_{\gamma} \mathrm{d} \mathbf{v}= \\
\int m_{\gamma} \mathbf{v} \sum_{\gamma^{\prime}}\left(\frac{\partial f_{\gamma}}{\partial t}\right)_{\gamma \gamma^{\prime}} \mathrm{d} \mathbf{v}+\int m_{\gamma} \mathbf{v} s_{\gamma} \mathrm{d} \mathbf{v}
\end{array}
$$

which after the integration of all possible terms and assuming that the external averaged force does not depends on the microscopic velocity, becomes

$$
\frac{\partial}{\partial t} \rho_{\gamma} \mathbf{u}_{\gamma}+\nabla_{\mathbf{r}} \cdot \mathbb{P}_{\gamma}-\frac{\mathbf{F}}{m_{\gamma}} \rho_{\gamma}=\int m_{\gamma} \mathbf{v} \sum_{\gamma^{\prime} \neq \gamma}\left(\frac{\partial f_{\gamma}}{\partial t}\right)_{\gamma \gamma^{\prime}} \mathrm{d} \mathbf{v}+\int m_{\gamma} \mathbf{v} s_{\gamma} \mathrm{d} \mathbf{v}
$$

where $\mathbb{P}_{\gamma}$ is known as the stress tensor, the term $\int m_{\gamma} \mathbf{v} \sum\left(\partial f_{\gamma} / \partial t\right)_{\gamma \gamma^{\prime}} \mathrm{d} \mathbf{v}$ is related to elastic exchanges among particles, excluding self-collisions as they do not modify the macroscopic values of $f_{\gamma}$. This equation can be further simplified [78, Chapter 8], but here we are only interested in the main effects that directly modify the flux of particles, as the external force, the exchange with other species or gradients in the stress tensor.

The evolution of each species energy, i.e., the second order moment, is obtained in a similar way, by integrating equation (3.1) with $m_{\gamma} v^{2} / 2$. Thence, there is an infinite hierarchy of equations that can be obtained from a distribution function, which means that an infinite number of equations should be stated. This issue is avoided by closing the equations up to some order of moment, usually up to second order, and making assumptions for the remaining parameters, leaving a system of $2+N$ fluid equations for each species $\gamma$ where $N$ is the dimension of the $\mathbf{r}$ space. This closure involves the socalled transport coefficients, relating forces and spatial gradients with typical fluxes. Kinetic calculations are usually employed to obtain these terms and close the fluid equations [43,69-72,74,91-93]. The accurate and correct derivation of these transport coefficients is of huge importance for fluid models, specially when complex systems, as plasmas or high enthalpy gases, are solved, increasing the complexity of the fluid codes.

\subsection{Collisionless plasma from a kinetic point of view}

A first approach to describe the dynamics of a plasma from a kinetic point of view consists on neglecting collisions and any other microscopic interaction among particles. These descriptions are widely used in situations where collisions are not dominant or the time and length scales studied are smaller than the collisional ones, meaning that its influence is marginal. However, it is important to stress out that collisions are always present and many situations may appear where these effects cannot be neglected and may drive the dynamics of the system $[19,25,94,95]$. Hence, the action of the electromagnetic field, or any other force, governs the dynamics of the system. Furthermore, non-homogeneous terms, as source-sinks of particles, or boundary conditions can also 
appear in the description of such plasmas. In this section the basic system of equations that describes non-collisional plasmas is presented. Some cases in the 1D1V dimensional planar space are studied to analyse the evolution of a plasma (or a gas) only affected by an external force, e.g., the self-consistent electric field.

Let us begin by analysing the general balance equation that describes the evolution of each species distribution function in a non-collisional regime. This balance is governed by the general Vlasov equation, supplemented by $s_{\gamma}$, recovered from (3.1)

$$
\frac{\partial f_{\gamma}}{\partial t}+\mathbf{v} \cdot \nabla_{\mathbf{r}} f_{\gamma}+\frac{q_{\gamma}(\mathbf{E}+\mathbf{v} \times \mathbf{B})}{m_{\gamma}} \cdot \nabla_{\mathbf{v}} f_{\gamma}=s_{\gamma} .
$$

This expression is widely used, in conjunction with the Maxwell's equations (3.9)(3.12), to describe many plasma behaviours, as the Landau dumping [96] or the noncollisional sheath $[97,98]$. It is easy to see how, in absence of non-homogeneous terms or boundary conditions, the distribution function evolution is defined by two convective effects, the electromagnetic force and the velocity $\mathbf{v}$.

Let us deal now with the $1 \mathrm{D} 1 \mathrm{~V}$ case, i.e., we constrain the evolution of the system to one dimension in space and its associated velocity. If planar geometry is assumed, in physical space, equation (3.17) becomes, with no magnetic effects,

$$
\frac{\partial f_{\gamma}}{\partial t}+v \frac{\partial f_{\gamma}}{\partial x}+\frac{q_{\gamma} E}{m_{\gamma}} \frac{\partial f_{\gamma}}{\partial v}=s_{\gamma}
$$

where $x$ is the position and $v$ is its associated velocity. The electric field comes from

$$
\frac{\partial \phi}{\partial x}=-E
$$

with appropriate boundary conditions, where $\phi$ is the electrostatic potential satisfying the Poisson's equation

$$
\frac{\partial^{2} \phi}{\partial x^{2}}=-\frac{\rho_{c}}{\epsilon_{0}}
$$

The system of equations (3.18) and (3.20) is solved numerically by means of the PIM for non-diffusive equations, as seen in 2.3.2, and the one for the one-dimensional Poisson's equation from 2.4.1. This numerical method provides the complete time evolving distribution function and its moments and fluxes as an output. To numerically solve the Vlasov equation, it is advisable to write (3.18) in non-dimensional form. To do so, each variable $h$ is factorised as $h=h^{0} \tilde{h}$, a reference $h^{0}$ and non-dimensional $\tilde{h}$ value. References values can be grouped as

$$
\frac{\partial \tilde{f}_{\gamma}}{\partial \tilde{t}}+\frac{v^{0} t^{0}}{x^{0}} \tilde{v} \frac{\partial \tilde{f}_{\gamma}}{\partial \tilde{x}}+\frac{q^{0} E^{0} t^{0}}{m^{0} v^{0}} \frac{\tilde{q}_{\gamma} E}{\tilde{m}_{\gamma}} \frac{\partial \tilde{f}_{\gamma}}{\partial \tilde{v}}=\frac{s^{0} t^{0}}{f^{0}} \tilde{s}_{\gamma}
$$

and in the Poisson's equation (3.20)

$$
\frac{\partial^{2} \tilde{\psi}}{\partial \tilde{x}^{2}}=-\frac{x^{0^{2}} q^{0} n^{0}}{\psi^{0} \epsilon_{0}} \tilde{\rho}_{c} .
$$


For the examples presented in this section, all scale factors equal one

$$
\frac{v^{0} t^{0}}{x^{0}}=1 ; \frac{q^{0} E^{0} t^{0}}{m^{0} v^{0}}=1 ; \frac{x^{0} q^{0} \tilde{n}}{\psi^{0} \epsilon_{0}}=1 ; \frac{s^{0} t^{0}}{f^{0}}=1 ; \psi^{0}=x^{0} E^{0} ; f^{0}=\frac{n^{0}}{v^{0}},
$$

and notation $\sim$ is dropped for the shake of clarity. This does not limit the applicability of the PIM to solve non-collisional plasmas but ease the implementation of numerical problems.

\subsubsection{Evolution of one species affected by a histogram-like constant force}

As a first testing case, we consider a species, of mass unity, that evolves with and without the action of an external histogram-like force. The initial distribution function is Maxwellian with temperature $T=1$ with the density distribution

$$
n_{0}(x)=e^{-\frac{x^{2}}{20}}
$$

The external force per unit of mass is defined as

$$
F(x, t)=\left\{\begin{aligned}
-1 & \text { if }-0.1 \leq x \leq 0.1 \\
0 & \text { elsewhere }
\end{aligned}\right.
$$

which takes the role of an electric field.

In figure 3.2 the distribution function in the phase-space is depicted. It can be seen how $f(x, v, t)$ evolves anti-symmetrically if no external force is applied, i.e., for particles with $v>0$ a motion to the right hand side is expected and in the opposite direction for $v<0$. Meanwhile, when the force (3.25) acts, the anti-symmetrical evolution is broken and differences for positive and negative velocities appear. The distribution function is modified only where $F$ is different from 0 . However, once the particles have exit this zone, they keep the velocity they have reached as a consequence of the acceleration process. Without collisions or other process that relax the species distribution function, a non-Maxwellian state is produced and carried out even outside $x \in[-0.1,0.1]$.

The system has a pure convective motion from the fluid point of view, also known as an Euler flow, due to the motion of particles at different velocities, i.e., pressure gradients, non interpreted as other diffusive effects, as Brownian motion, where the stochastic nature of the diffusive tensor has an influence in all the space, as seen in section 2.2.2. Without boundary restrictions, this motion results in all the initial condition expanding in space until density reaches a zero value at $t \rightarrow \infty$.

The flux of particles [1] $=n u$ for the cases with and without external force is presented in figure 3.3. The non-symmetrical evolution when the external force actuates is clearly detected. The deceleration caused by $F$ translates into a deeper zone of negative $n u$ in 


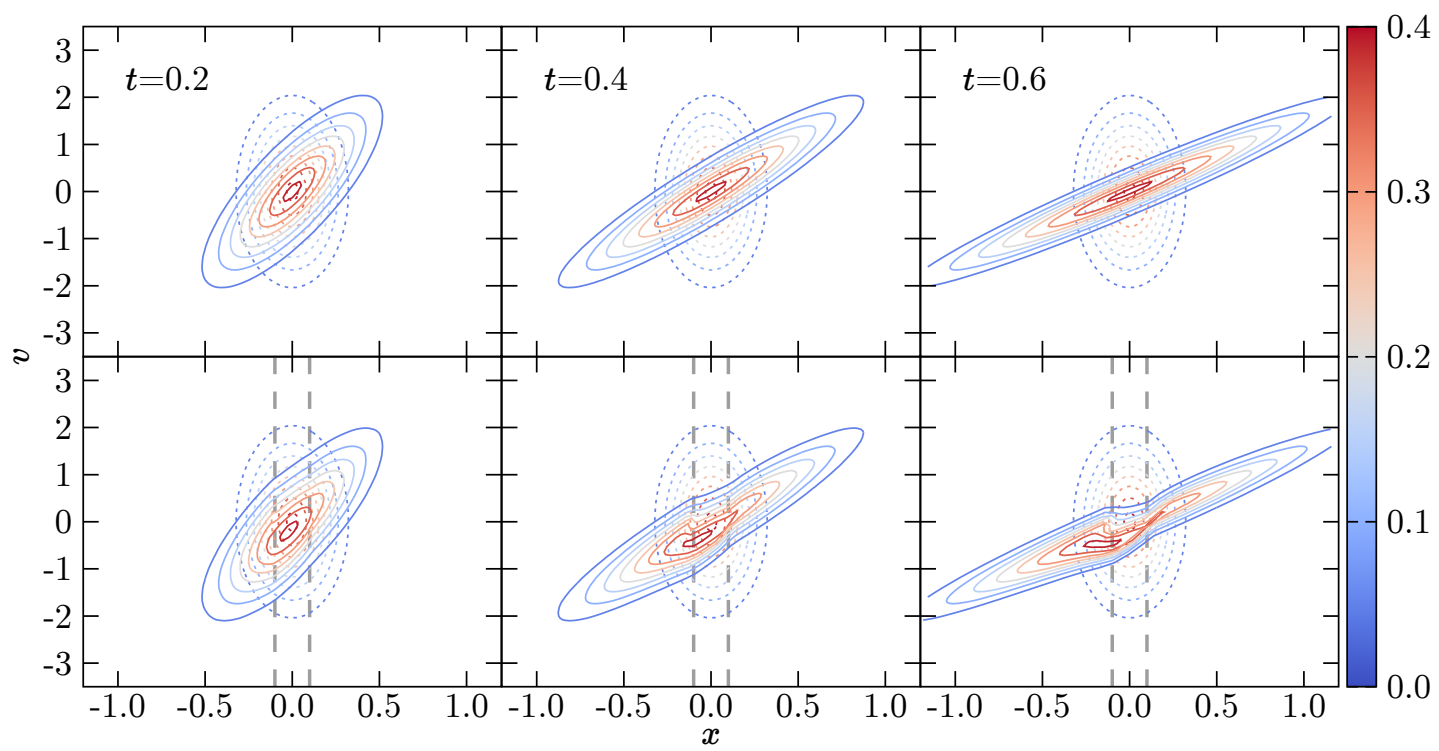

Figure 3.2: Evolution of the distribution function in the phase-space. Top row: without external force. Bottom row: with the external field defined in (3.25). Grey dashed lines represent the action zone of the external force. Dashed contours represent the initial condition.

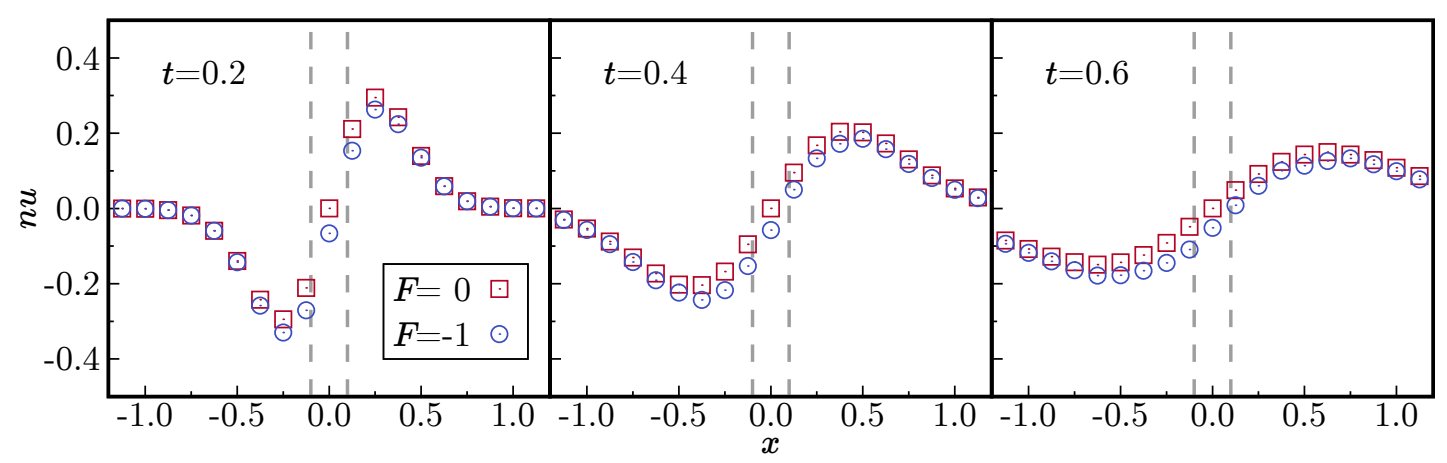

Figure 3.3: Flux of particles with and without the external histogram-like force.

the surroundings of $x \pm 0.1$. Moreover, as time increases, the area decelerated by the external force grows, as more particles dynamics are modified by it.

Furthermore, differences in the fluxes indicate that this force has the power to retain particles and increase its density for $x<-0.1$, as can be seen in figure 3.4, where the norm $\langle 1\rangle=n$ is pictured. The density of particles reaches a higher value for negative positions, while is reduced for $x>0.1$, respect to the case without force. This effect is qualitatively similar to what happens in the acceleration grid of an ion thruster [99,100], where, due to the intense electric field, generated by means of polarised grids, the ions are exhausted at high speed to generate thrust and the electrons are slowed down to keep them inside the discharge chamber and contribute to the ionization process.

In resume, the PIM is able to reproduce this and other cumbersome behaviours based only on the self-consistent resolution of the complete distribution function in the phasespace. With this simple example, the PIM for non-diffusive equations has been tested in 


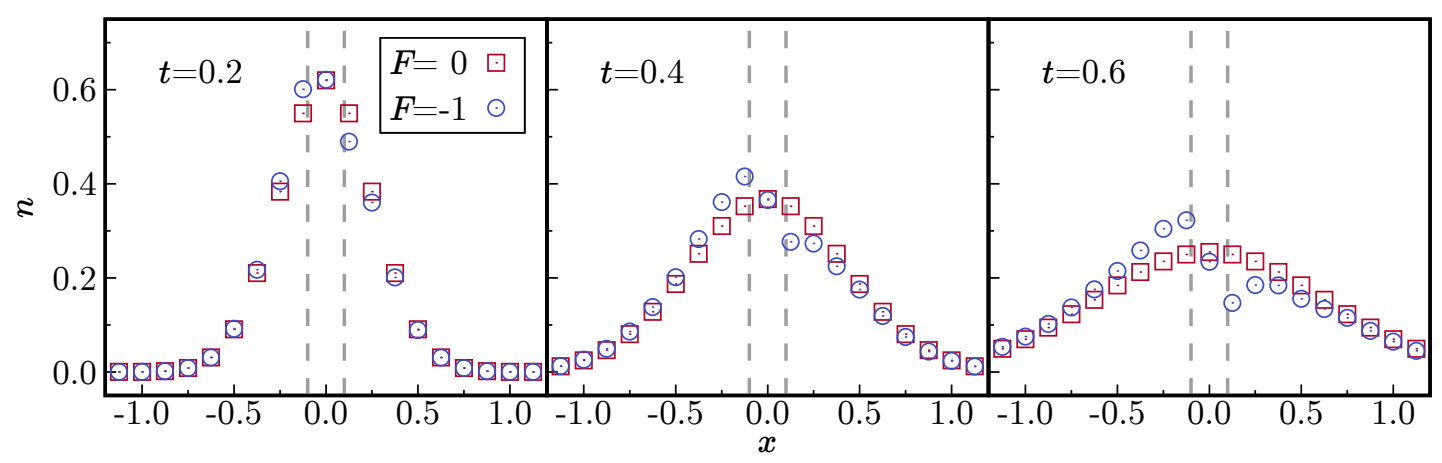

Figure 3.4: Particle density with and without the external histogram-like force. A small amount of particles is retained for $x<-0.1$.

an $x-v$ phase space with an histogram-like external force. The addition of extra species to the problem is quite simple because no microscopic exchanges among them appears, so it is just a matter of solving the new Vlasov equation for the extra species in a similar way, accounting for possible difference in charge, mass, density or temperature ratios. Also, the coupled Poisson's equation must be introduced to account for the possible charge separation among the different species.

\subsubsection{Two charged species with self-consistent electric field}

After the previous test case, here the evolution of a plasma composed by two species under the effect of the self-consisted electric field in a $1 \mathrm{D} 1 \mathrm{~V}$ phase-space is studied. By self-consistent electric field we refer here to the one obtained through Maxwell equations and influenced only by boundary conditions or charge-separation. Here, the electric field is obtained by means of the Poisson's equation with the method explained in section 2.4.1. The two test cases solved here are representative of relevant physical events, as for example the Landau damping [96], and allows the study from a fully kinetic point of view, without employing perturbation methods or approximations to solve the distribution function evolution.

In these examples, two species of opposite charges, mass ratio of 0.001 and temperature ratio of 10 are advanced. This means that there is a light species of mass 1 , charge -1 and temperature 1, representing electrons; and a heavy species of mass 1000, charge 1 and temperature 0.1, representing ions. All values are expressed in dimensionless units. The convective evolution in the $x-v$ space is independent for each species, due to differences in temperature and mass, which affect the thickness of the distribution function, and the different acceleration of both species due to the electric field, related to each species mass. It is expected that these different dynamics generate a charge separation, modifying the electric field. Nevertheless, the PIM allows to advance both species with the same time step without perturbing any of the species dynamics and providing a stable and physical meaningful evolution. 


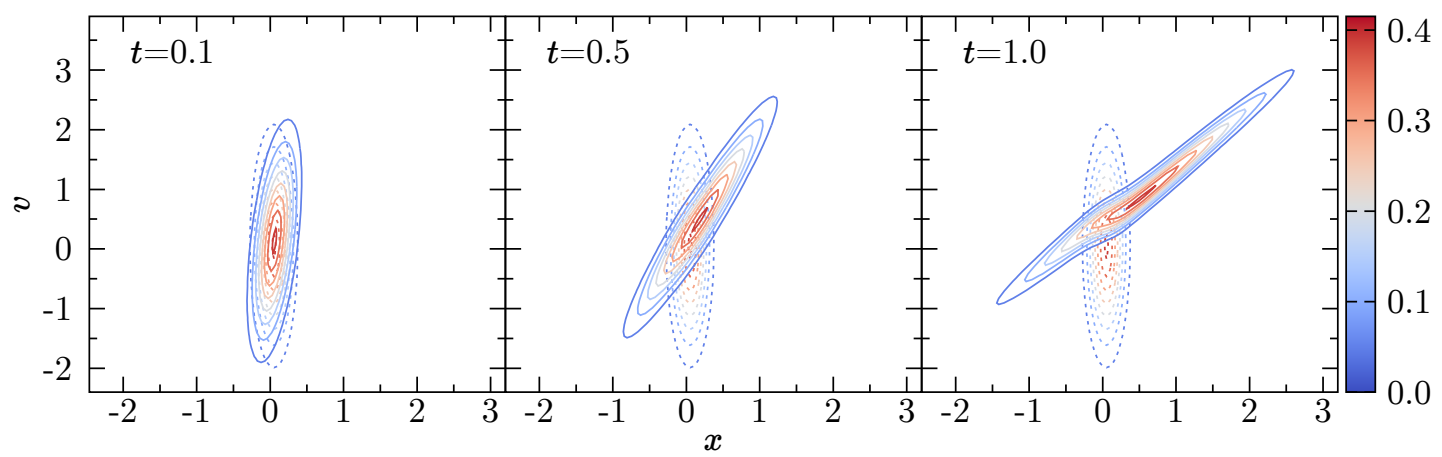

Figure 3.5: Evolution of electrons under a self-consistent electric field between a positive potential jump. Dashed contours represent the initial condition.

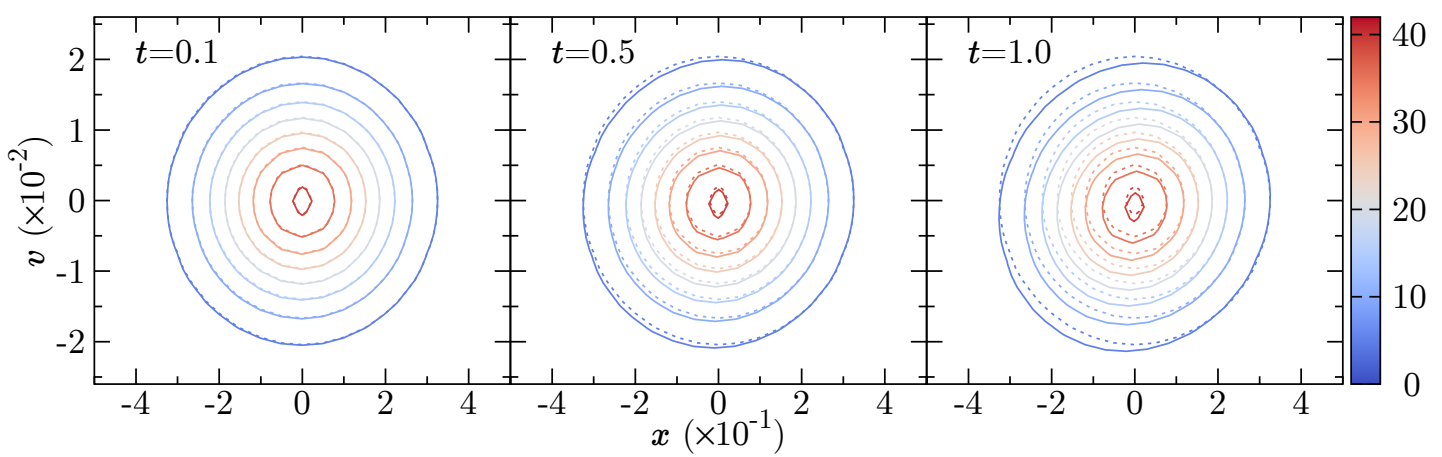

Figure 3.6: Evolution of ions under a self-consistent electric field between a positive potential jump. Dashed contours represent the initial condition.

Firstly, the case with a positive potential difference between two points, i.e., $\phi(-10)=$ -10 and $\phi(10)=10$ in dimensionless units, is presented. This means a negative electric field arise in all the domain, except if a strong charge separation appears. Therefore, electrons are accelerated positively and ions negatively through all the simulations. Without collisions, these acceleration processes are the dominant phenomena in the species evolutions. A perturbation in the electrons initial density and velocity as

$$
n_{e}(x)=\exp \left[-\frac{(x-0.05)^{2}}{0.05}\right], u_{e}(x)=0.05
$$

is used to start from a non-constant electric field. Ions have the same density distribution but centred at $x=0$ and zero mean velocity for all positions.

Figures 3.5 and 3.6 present the evolution of the distribution function of electrons and ions, respectively. It can be seen how electrons evolve in a fast time scale and escape from the initial condition under the negative electric field. This force produces a positive density particle flux in the evolution of electrons in almost all the domain, figure 3.7. Nevertheless, due to the relative large amount of particles that start with a velocity lower than zero, electron flux is negative for a small section at $x<0$. As time increases, this area becomes less negative, because electrons drift to a positive velocity, leaving macroscopic particle flux positive in all the domain. 


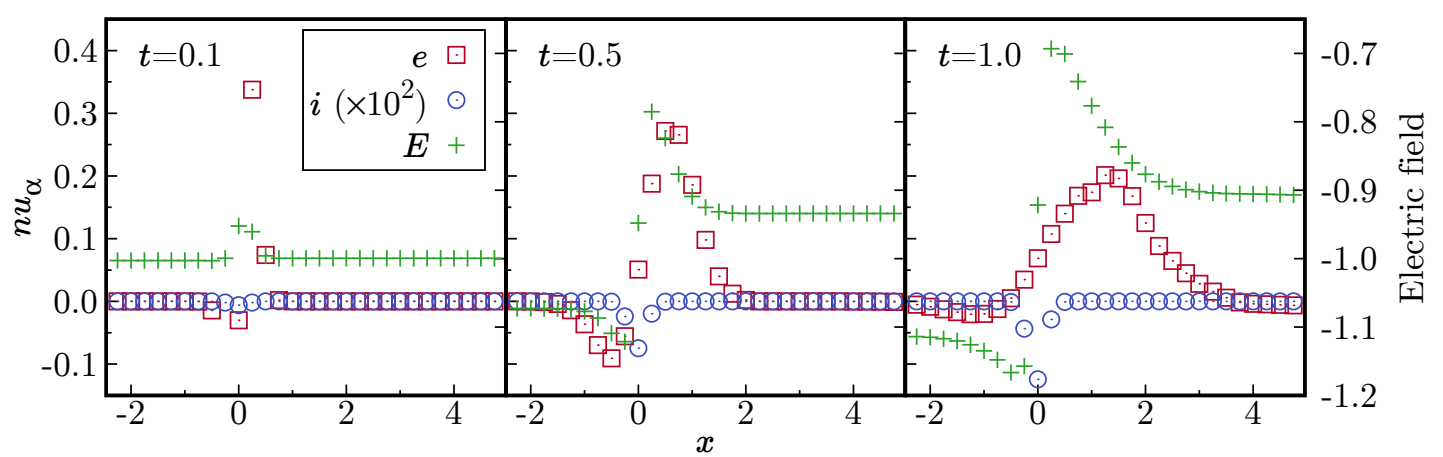

Figure 3.7: Fluxes for electrons (red squares) and ions (blue circles) on the left axis. Electric field (green crosses) on the right axis. Ions flux multiplied by a factor of $10^{2}$ to appreciate its evolution.

Ion distribution function does not seem to change and only a small flux appears due to the action of the electric field. The value of this flux is in the order of magnitude of $m_{e} / m_{i}$ respect to the electron flux. This can lead us to assume that ion dynamics could be neglected and only the electron evolution should be computed. However, it is important to remember that small changes of the ion particle density influence the resolution of the Poisson's equation, which modify the electron motion due to their low mass, specially in the analysis of long times [21,101]. So, if there are enough computational resources, it is advisable to advance both species, also with the same time step, to not perturb the problem and to obtain a more accurate electric field. Furthermore, if the sign of the imposed potential jump were inverted, it is expected that evolution of ions and electrons will be very similar but with opposite acceleration.

The flux of particles $n u_{\alpha}$, being $\alpha=e, i$, is now analysed, where the different evolution of both species is easily comprehended. Figure 3.7 shows the flux of electrons and ions in the left axis and self-consistent electric field in the right one. Here, ion evolution is clearly identified, but in a slower time scale, due to their high mass and low temperature. Nevertheless, this evolution is important to accurate compute the electric field through the Poisson's equation and to describe the dynamics in larger time scales. It can be seen how the negative self-consistent field accelerates species in opposite directions, creating a strong charge separation. The electric field obtained has a very abrupt change close to the point where the charge density changes its sign. It is important to remark that this abrupt change do not affect the stability of the solution and do not introduce any numerical errors. Moreover, the non-linear electric field formed means that particles are accelerated at different rates, depending on its position. For example, particles with position lower than zero feel a more intense electric field. However, on the right hand side of the domain, electric field is less intense and particle acceleration is reduced. This can create an avalanche phenomena, where particles at a more negative position are highly accelerated until they overpass particles that started at a more positive initial position and velocity. The conditions of this avalanche to appear arise naturally from the self-consistent resolution of the Vlasov-Poisson system. The study of this phenomena will be left for future works, but we can point out here its importance in 


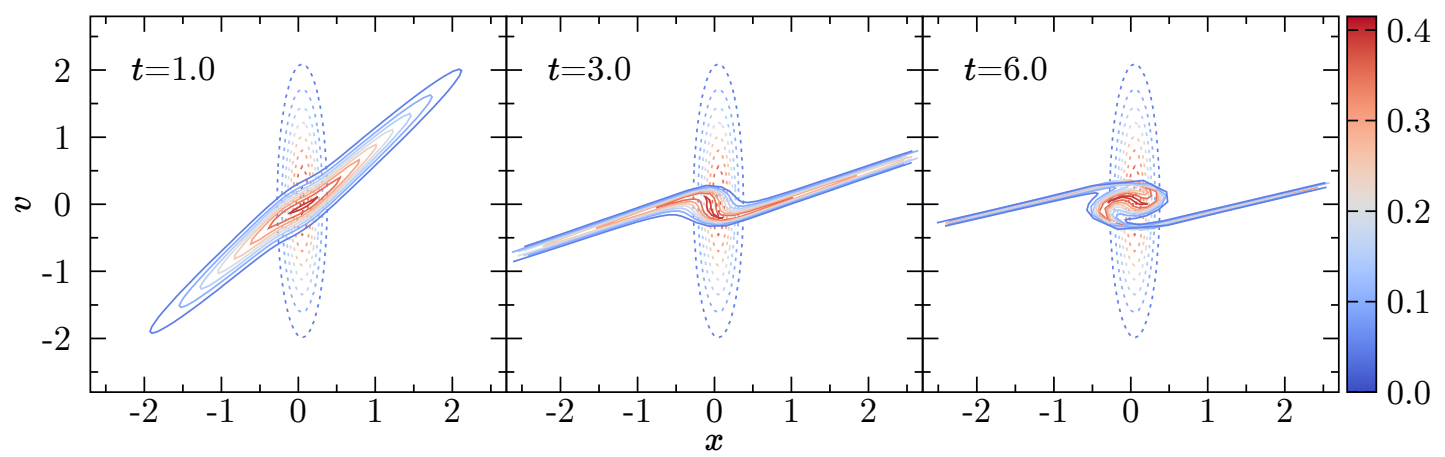

Figure 3.8: Evolution of electrons a under self-consistent electric field with no potential difference at the boundaries. Dashed contours represent the initial condition.

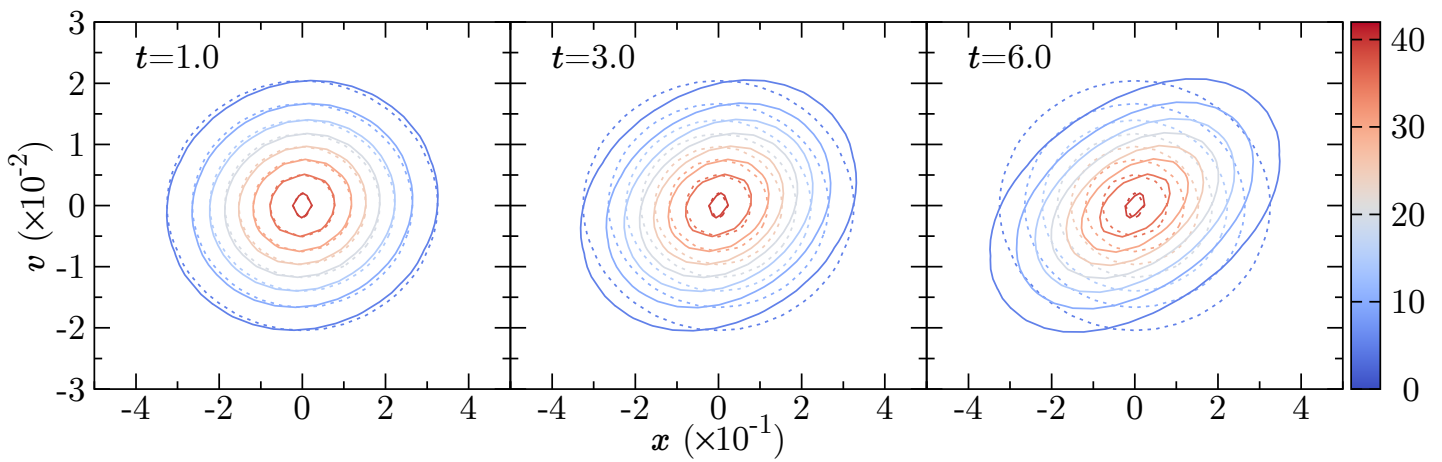

Figure 3.9: Evolution of ions under a self-consistent electric field with no potential difference at the boundaries. Dashed contours represent the initial condition.

several phenomena, as arc formation. Nevertheless the self-consistent resolution by means of the PIM takes a new approach to solve non-collisional plasmas, not available before.

To continue our discussion exploring the capability of the PIM to solve non-collisional plasmas, a case with no potential difference between the minimum and maximum points is presented. In this case, the electric field is produced exclusively by the difference of electron and ion densities, i.e., charge separation, and, in principle, particles should not be accelerated at infinity as a de-compensation in the charge density generates an electric field that tends to reduce this effect. For example, if a large amount of electrons appears on the right hand side, a positive electric field will arise, decelerating the electrons and reducing the charge separation. This will leave the plasma in an oscillatory state, related to the characteristic plasma oscillations of frequency $\omega_{p e}[78$, $102]$.

Thence, figure 3.8 draws the electron distribution function at different time frames. Same goes for ions in figure 3.9. Complete different dynamics respect to the case of a positive potential jump presented above are found. In this situation, the electrons evolve until an almost cyclical behaviour appears. Moreover, $f_{e}$ twist around $x, v \simeq 0$, while particles keep rotating around this centre, creating a zone of higher electron density in the centre and some low density tails far from $x=0$. This behaviour influences 


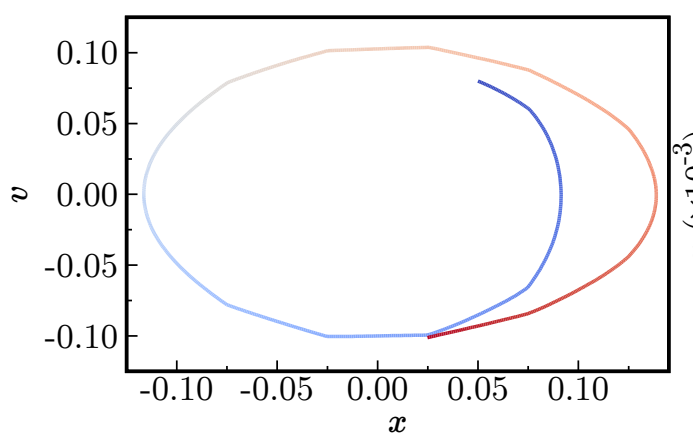

(a) Electrons

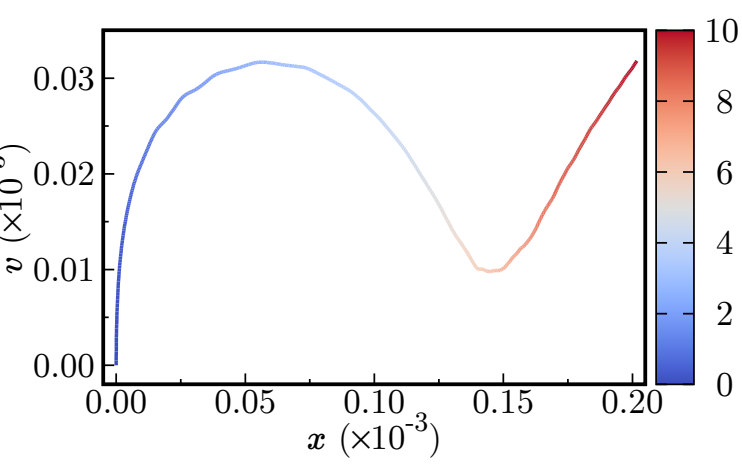

(b) Ions

Figure 3.10: Electron and ion maximum distribution point trajectory.

the electric field through the self-consistent Poisson's equation. A similar rotational ion dynamics is expected, because the electric field also influence the evolution of this species. However, slow ion variation, as well as the fast motion of the electrons, can give rise situations where electric field changes its sign and ions have not reached a periodical behaviour, creating a non-circular dynamics for this heavy species, which may end up affecting the evolution of electrons due to the changes in the charge density [101]. This is better appreciated in figure 3.10, where the trajectory of the maximum point of $f_{\alpha}$ is pictured. The evolution of the maximum distribution point depends on the initial conditions, i.e., if the species has some initial perturbation, and on the acceleration, proportional to $q_{\alpha} E(x, t) / m_{\alpha}$. Electrons recover an almost circular dynamics after a time of $t \simeq 10$, while ions have an almost periodical evolution, but not cyclical. However, in order to relate these dynamics to the characteristic frequencies of electrons and analyse the influence of ions dynamics, longer simulation times should be solved, which requires computational resources unavailable at this moment. Nevertheless, results pictured here evidence a promising method, not founded on perturbation or wave theory, to detect non-trivial plasma dynamics. Moreover, this example sets a great test of the PIM's capability to solve complex non-collisional self-consistent problems. Different initial perturbations and mass ratios should be analysed too, to study their effect in the plasma dynamics.

The particle flux for each species $n u_{\alpha}$ and the electric field are pictured in figure 3.11. It can be seen how at time $t=1$, the electron's particle flux and the electric field are negative directed for $x<0$ and positive directed for $x>0$. The action of the electric field changes this situation leading to inversion of the flux sign at $t=3$. On the other hand, the shape of ions flux remains almost unaltered and its is only slightly affected by the electric field. As it happens with the previous case, an abrupt variation in the electric field also appears close to $x=0$, which changes the species dynamics according to its position in $x$. Rather than in the previous example, here the electric field also changes its sign for in the surroundings of $x=0$, which tries to retain particles to reduce the charge separation. This could lead to a complete sign inversion and a periodical motion of electrons, known as Landau damping [96,103]. 


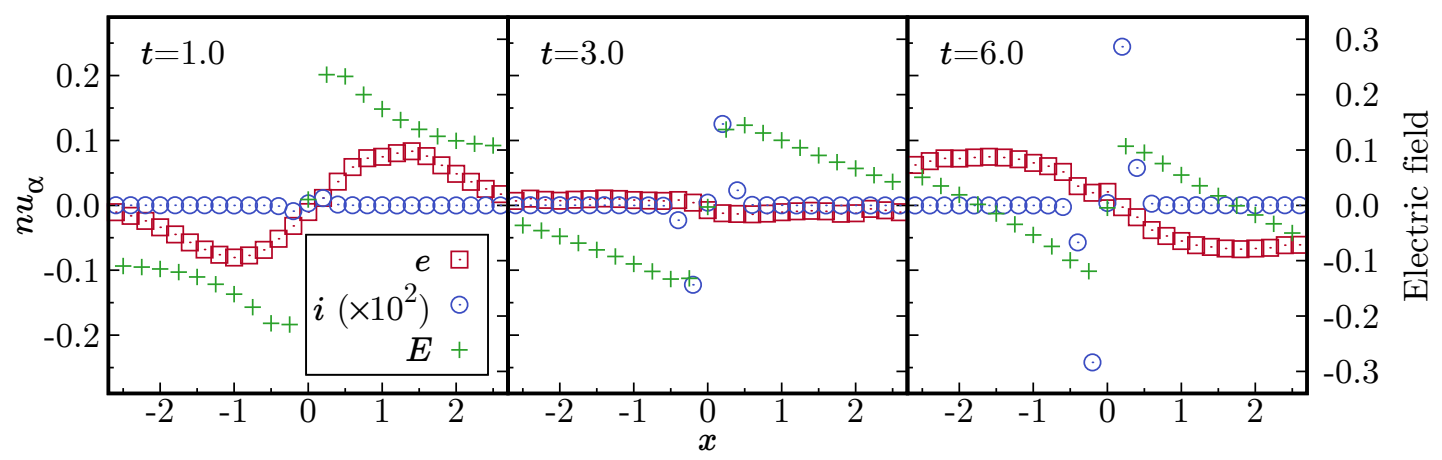

Figure 3.11: Evolution of fluxes under a self-consistent electric field with no potential difference at the boundaries. Ions flux multiplied by a factor of $10^{2}$ to appreciate its evolution.

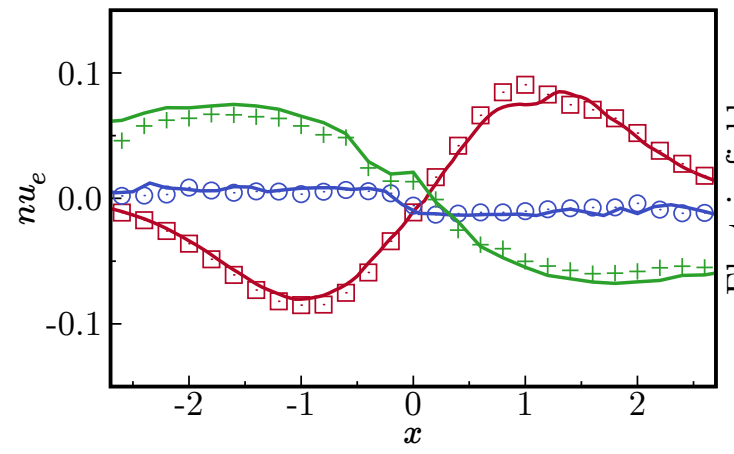

(a) Electron particle flux

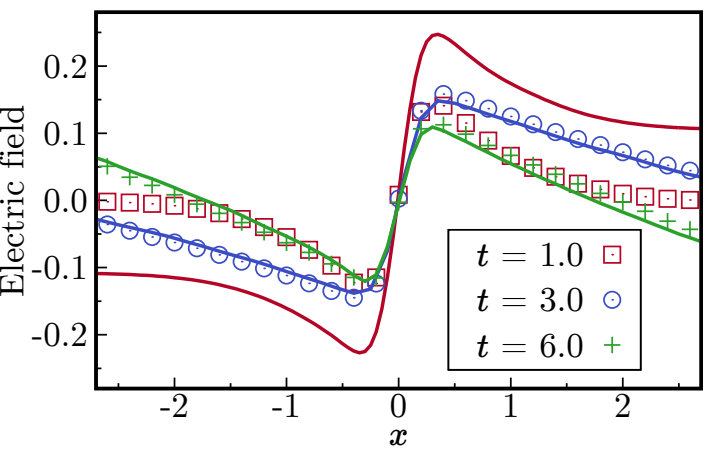

(b) Electric field

Figure 3.12: Evolution of electron flux (left) and self consistent electric field (right) for fixed ions. Solid lines represents the case where ions are also evolved in time. Small differences between both cases arise.

Finally, a last check is still to be performed: analyse the effect of the movable ions, i.e., the differences found when evolution of ions is computed at each time step and when they are fixed. In simulations and analytical models, it is commonly to assume that heavy species, as ions, are fixed, neglecting its evolution and acting only as a background. This affects the shape of the electric field, which influences the electrons dynamics. Now, the problem of two charged species interacting under a self-consistent field without a potential difference at the boundaries presented above is analysed setting the ions to its initial condition for all the simulation. Figure 3.12 compares the electron flux and the self-consistent electric field with and without accounting for the evolution of ions. Note how small differences in the electron flux and electric field appear, even when the shape of the time evolving solution holds. Nevertheless, these small differences could lead to different transitory situations or even disparate steady states in many systems of physical interest.

As a conclusions, it is important, if it is numerically possible, to compute the evolution of all heavy species, because small changes in its dynamics may influence the value and shape of the electric field and, as a consequence, the dynamics of electrons. 


\subsection{Conclusions}

In this chapter, the basic equations that describe the evolution of a distribution function under the Kinetic Theory point of view have been presented. In addition, a short summary of fluid equations is given to establish a common ground to understand the evolution of macroscopic quantities and to provide a brief resume of the importance of transport coefficient calculations for fluid models. Some cases of non-collisional plasmas calculated with the Propagator Integral Method have been given.

A case of a single species evolving with and without a histogram-like force results useful to understand the basic motion in the phase-space. In absence of collisional process, distribution functions may reach situations far from Maxwellian equilibrium.

The case of two opposite charged species evolving under a self-consistent electric field, determined through the coupled Poisson's equation, was also presented. The two species, that represent electrons and ions, have huge mass and temperature ratios, but the PIM can use the same time step to solve both evolutions. Each species evolves independently in the $x-v$ space accounting for their own characteristics. The potential difference between the boundaries determines the evolution of both species, reaching quasi-periodical situations if no difference is imposed, which means that the problem auto-compensate the charge differences. Physical behaviours are reproduced with this semi-analytical method, based only on a self-consistent resolution of the distribution function for each species. Nevertheless, numerical issues related with the interpolation into a fixed mesh to allow the integration of macroscopic moments introduce some instabilities into the fluid quantities, that perturb the analysis of larger time scales.

These topics require further investigation to optimise and increase the solutions accuracy, but the cases presented here open the possibility of analyse non-collisional plasmas to check if some typical processes, as Landau damping, arise naturally. Hence, the nondiffusive propagator has been tested in physical meaningful scenarios, providing some results, arduous to obtain with other numerical methods or perturbation theory. 


\section{CHAPTER 4}

\section{DESCRIPTION OF PARTICLE COLLISIONS}

Collisions among particles in a plasma are of huge importance to describe its evolution from kinetic and fluid points of view. Moreover, they are the main cause of energy and momentum exchange among the mixture of species as well as they are responsible of thermalisation and transport processes. Within the scope of kinetic theory, the collision term represents a contribution to the rate of change of $f_{\gamma}$ from a time instant $t$ to a time $t+\mathrm{d} t$ due to interaction with particles of the species $\gamma^{\prime}$, where $\mathrm{d} t$ is a differential time interval. Collisional terms include a variety of process, as elastic energy exchanges, ionization and recombination processes or charge-exchange effects. In this chapter, analytical and numerical analysis of the exchange phenomena employed in the forthcoming chapters are presented. Firstly, elastic collisions are studied. By elastic we refer here to those operators that conserve the total norm, energy and momentum of all species involved in the collisional process. Special attention is given to the conservation of total energy and momentum, which is of major importance to ensure that trustworthy numerical results are computed. It is important for the application of a numerical method that the conserved properties of the collision operators are fulfilled also in the numerical problem. All operators are studied for the self-interacting collisional case, i.e., $\gamma=\gamma^{\prime}$ to analyse the conservation properties. However, it is very common, specially in Weakly Ionized Plasmas (WIP), that disparate ratios between the species properties exist, which highly influence the collisional processes. Thence, binary elastic collisions with large mass, temperature and density ratios among the species is studied for the one-dimensional collisional terms.

As a relevant case, the influence of self-collisions when a neutral gas evolves from discontinuous initial macroscopic variables in a $x$ - $v$ phase-space is solved. A collisionless scenario and two collisional cases are compared to identify the differences in macroscopic moments and distribution function evolutions. In addition, the study of the charge- 
exchange phenomena between ions and neutrals is performed. This process becomes significant in many systems, e.g., in the analysis of an electric thruster plume presented in chapter 7 .

All the problems dealt in this chapter are solved in dimensionless units. However, this does not limit the applicability of Propagator Integral Method but ease the numerical implementation and results analysis.

\subsection{Elastic collisions}

Elastic collisions denote the exchange of energy and momentum between species without loosing any of the system total energy, i.e., the sum of all species energies involved in the collisional processes remains constant. These phenomena are the main cause of thermalisation and transport of mass, momentum and energy in plasmas [104, Chapter 1.5]. In this section, the different collision terms employed through out this dissertation are separately analysed. Furthermore, it is important to explore these terms to identify some behaviours that may arise in scenarios where collisional effects are integrated into more involved models. Moreover, the conservation properties of the collision terms for the self-interaction situation are studied, an important attribute that helps to analyse the numerical method validity. If macroscopic quantities are numerically conserved, as happens in the analytical case, then the numerical method can be considered valid to analyse collisional problems. Except for very specific systems, out of the scope of this work, plasmas are usually composed by a mixture of species that evolve and interact among them. To describe these interactions from the point of view of elastic collisions, theoretical derivation by means of the binary interactions approach are usually utilised, neglecting more complex interactions that involve a larger number of particles and are infrequent. In these exchanges, the different properties of the species, as charge, mass or density, modify the collisional processes, influencing the system dynamics. For these reasons, it is important to study the binary exchange, specially if high ratios of mass, density or temperature exist between the two species. Usually, to solve these interactions, simplifications or linearisations are employed to simplify the collision terms. For example, it is very common that, for an electron-ion elastic collision, the ratio of masses is neglected because $m_{e} / m_{i} \ll 1$, which may introduce small differences in the dynamic of both species and in the total conservation of energy. Nevertheless, even these ratios are small, they are far from being zero, and they can affect the relaxation times, transport coefficients and conservation of energy.

For our analysis, a test particle of class $a$ interacts with a $b$ class particle. Properties of both species are in table 4.1 and Maxwellian distributions functions are assumed for the initial condition. When binary interaction approximations are analysed, self-collisions are not included, to study the isolated exchange between species. 


\begin{tabular}{ccccc}
\hline & $m$ & $n$ & $u$ & $T$ \\
\hline$a$ & 1 & $10^{-3}$ & 1 & 1 \\
$b$ & $10^{3}$ & 1 & 0 & 0.01 \\
\hline
\end{tabular}

Table 4.1: $\quad$ Mass, density, velocity and temperature for the two species studied in the binary collision.

\subsubsection{The Fokker-Planck-Landau (FPL) term}

In plasmas, collisions among charges in fully ionized scenarios are usually described by the well-known Fokker-Planck-Landau operator [43, 78, 82, 104-106]. This term is usually assumed as an approximate to the Boltzmann collision integral operator, but it can be derived independently by physical arguments, as presented by B. A Trubnikov [82]. This integro-differential operator describes the drifting and scattering of a charged particle by interparticles Coulombian interactions. The expression of this collision term adopts the form of a Fokker-Planck equation $[78,104]$ as

$$
\frac{\partial f_{\gamma}}{\partial t}=-\sum_{\gamma^{\prime}} \frac{\partial}{\partial v_{i}}\left[D_{i}^{\gamma \gamma^{\prime}}-\frac{\partial}{\partial v_{j}} D_{i j}^{\gamma \gamma^{\prime}}\right]
$$

where coefficients $D_{i}^{\gamma \gamma^{\prime}}$ and $D_{i j}^{\gamma \gamma^{\prime}}$ are the convective and diffusion parameters and $i, j$ represent their components axes of the velocity space.

The drift and diffusion coefficients required by (4.1) can be expressed as $D^{\gamma \gamma^{\prime}}=$ $\int d(g) f_{\gamma^{\prime}}^{\prime} \mathrm{d} \mathbf{v}^{\prime}$ where $d$ are the kernels

$$
d_{i}^{\gamma \gamma^{\prime}}=-\left(1+\frac{m_{\gamma}}{m_{\gamma^{\prime}}}\right) \frac{l \gamma \gamma^{\prime}}{4 \pi} g \frac{g_{i}}{g^{\frac{4}{n}}}, d_{i j}^{\gamma \gamma^{\prime}}=\frac{l^{\gamma \gamma^{\prime}}}{8 \pi} g \frac{\delta_{i j} g^{2}-g_{i} g_{j}}{g^{\frac{4}{n}}}
$$

being $\mathbf{g}=\mathbf{v}-\mathbf{v}^{\prime}$ the relative velocity and $g$ its module, $l^{\gamma \gamma^{\prime}}$ is usually a constant related to the characteristic length of the interaction between the species and $n$ comes from the general interaction potential between particles $V(\mathbf{r})=k r^{-n}$, where $k$ is a constant and $n=1$ for Coulombian case.

As stated in section 2.2.3, an interesting case in Plasma Physics is the study in the two velocity space composed by velocities perpendicular $\perp$ and parallel $\|$ to the electric or magnetic field. Accounting for the cylindrical geometry with the velocity volume element $\mathrm{d} \mathbf{v}=v_{\perp} \mathrm{d} v_{\perp} \mathrm{d} v_{\|} \mathrm{d} \phi$, the drift-diffusion parameters are simplified to

$$
\begin{gathered}
D_{\perp}^{\gamma \gamma^{\prime}}=-\left(1+\frac{m_{\gamma}}{m_{\gamma^{\prime}}}\right) \frac{l \gamma \gamma^{\prime}}{4 \pi} \int \frac{g_{\perp}}{g^{3}} f_{\gamma^{\prime}}^{\prime} \mathrm{d} \mathbf{v}^{\prime} ; D_{\|}^{\gamma \gamma^{\prime}}=-\left(1+\frac{m_{\gamma}}{m_{\gamma^{\prime}}}\right) \frac{l \gamma \gamma^{\prime}}{4 \pi} \int \frac{g_{\|}}{g^{3}} f_{\gamma^{\prime}}^{\prime} \mathrm{d} \mathbf{v}^{\prime} ; \\
D_{\perp \perp}^{\gamma \gamma^{\prime}}=\frac{l \gamma \gamma^{\prime}}{8 \pi} \int \frac{g^{2}-g_{\perp} g_{\perp}}{g^{3}} f_{\gamma^{\prime}}^{\prime} \mathrm{d} \mathbf{v}^{\prime} ; D_{\|\|}^{\gamma \gamma^{\prime}}=\frac{l \gamma \gamma^{\prime}}{8 \pi} \int \frac{g^{2}-g_{\|} g_{\|}}{g^{3}} f_{\gamma^{\prime}}^{\prime} \mathrm{d} \mathbf{v}^{\prime} ; \\
D_{\phi \phi}^{\gamma \gamma^{\prime}}=\frac{l^{\gamma \gamma^{\prime}}}{8 \pi} \int \frac{g^{2}-g_{\phi} g_{\phi}}{g^{3}} f_{\gamma^{\prime}}^{\prime} \mathrm{d} \mathbf{v}^{\prime} ; D_{\perp \|}^{\gamma \gamma^{\prime}}=-\frac{l \gamma \gamma^{\prime}}{8 \pi} \int \frac{g_{\perp} g_{\|}}{g^{3}} f_{\gamma^{\prime}}^{\prime} \mathrm{d} \mathbf{v}^{\prime} ;
\end{gathered}
$$


where $f^{\prime}=f\left(\mathbf{v}^{\prime}, t\right)$ and the components of the relative velocity vector $\mathbf{g}$ are

$$
\begin{aligned}
g_{\perp} & =v_{\perp}-v_{\perp}^{\prime} \cos \phi^{\prime}, \\
g_{\|} & =v_{\|}-v_{\|}^{\prime}, \\
g_{\phi} & =-v_{\perp}^{\prime} \sin \phi^{\prime},
\end{aligned}
$$

giving

$$
g^{2}=\left|\mathbf{v}-\mathbf{v}^{\prime}\right|^{2}=v_{\perp}^{2}-2 v_{\perp} v_{\|}^{\prime} \cos \phi^{\prime}+v_{\perp}^{\prime 2}+\left(v_{\|}-v_{\|}^{\prime}\right)^{2} .
$$

These integrals over $\mathrm{d} \phi^{\prime}$ can be expressed as elliptic integrals

$$
I(n)=\frac{1}{4 \pi}\left[\frac{m}{2 v_{\perp} v_{\perp}^{\prime}}\right]^{\frac{3}{2}} \int_{0}^{\pi} \frac{\cos ^{n} \phi^{\prime}}{\left[1-m \cos \phi^{\prime}\right]^{\frac{3}{2}}} \mathrm{~d} \phi^{\prime}
$$

where $m=\frac{2 v_{\perp} v_{\perp}^{\prime}}{v_{\perp}^{2}+v_{\perp}^{\prime 2}+\left(v_{\|}-v_{\|}^{\prime}\right)^{2}}$ being $0 \leq m \leq 1$ a functional parameter [43].

The conservation of momentum in the binary exchange is fulfilled if $\left\langle m_{\gamma} D_{\|}^{\gamma \gamma^{\prime}}\right\rangle=$ $-\left\langle m_{\gamma^{\prime}} D_{\|}^{\gamma^{\prime} \gamma}\right\rangle$, where \langle\rangle represents the integration over the distribution function, which is true if $l^{\gamma^{\prime} \gamma}=m_{\gamma}^{2} / m_{\gamma^{\prime}}^{2} l l^{\gamma \gamma^{\prime}}$ [104, Chapter 1.5]. In the same way, energy conservation is fulfilled as $m_{\gamma}\langle\operatorname{Tr}(\mathbb{D})+\mathbf{v} \cdot \mathbf{D}\rangle_{\gamma \gamma^{\prime}}=-m_{\gamma^{\prime}}\langle\operatorname{Tr}(\mathbb{D})+\mathbf{v} \cdot \mathbf{D}\rangle_{\gamma^{\prime} \gamma}$. In the case of self-collisions $\gamma=\gamma^{\prime}$, the conservation properties are fulfilled for any value of $l^{\gamma \gamma}$. The $\gamma$ notation is dropped for the self-collisional case for the shake of clarity.

To numerically analyse the problem of self-collisions, an initial histogram-like distribution function is employed as

$$
\begin{aligned}
f_{0}\left(v_{\perp}, v_{\|}\right)= & \frac{n}{8 \pi T_{\perp} / m \sqrt{2 T_{\|} / m}}\left[1-H\left(v_{\perp}-\sqrt{4 T_{\perp} / m}\right)\right] \\
& {\left[H\left(v_{\|}-u_{\|}+\sqrt{3 T_{\|} / m}\right)-H\left(v_{\|}-u_{\|}-\sqrt{3 T_{\|} / m}\right)\right] }
\end{aligned}
$$

where $H(x)$ is the Heaviside function

$$
H(x)=\left\{\begin{array}{ll}
1 & \text { if } x>0 \\
0 & \text { if } x \leq 0
\end{array},\right.
$$

defined in this form to simplify its numerical evaluation, $u_{\|}, T_{\perp}$ and $T_{\|}$are the mean transversal and parallel kinetic temperatures, being $3 T=2 T_{\perp}+T_{\|}$the total temperature; and $m$ is the particle mass. The initial conditions for the self-collisional case are $n=1, T_{\perp}=1.07625, T_{\|}=0.775, u_{\|}=0.5$ and $m=1$ is the species mass; in dimensionless units, where $l^{\gamma \gamma}$ equals $8 \pi$.

The two-dimensional distribution function contours evolution is pictured in figure 4.1, with the initial condition as a black solid line. The distribution function evolves smoothly from the histogram-like to a Maxwellian one with no numerical instabilities. This occurs thanks to the global effect of the diffusive process, i.e., the value 


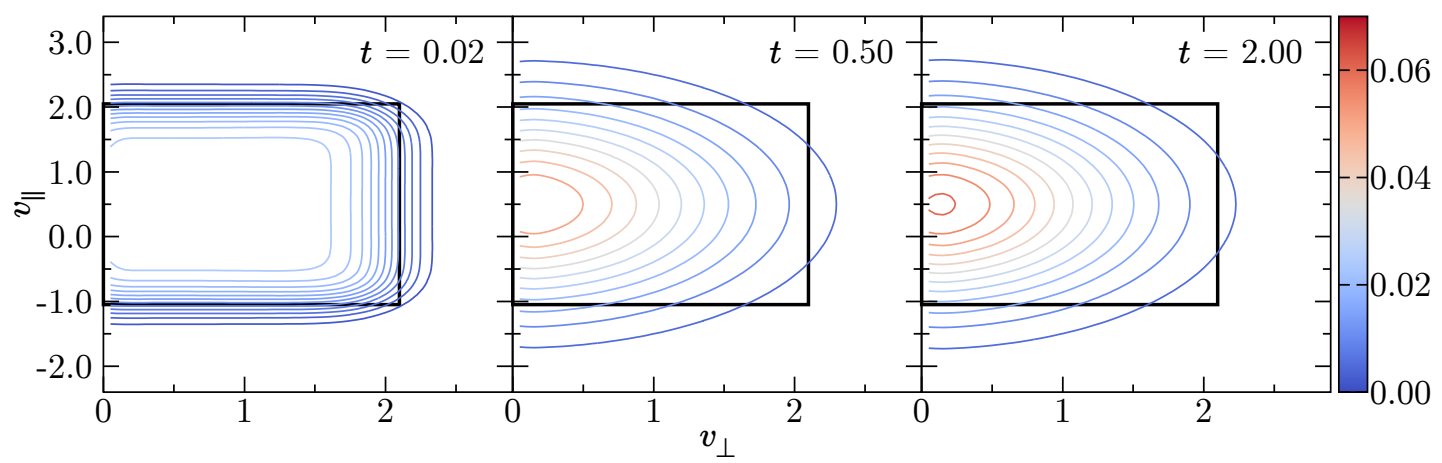

Figure 4.1: Distribution function $f\left(v_{\perp}, v_{\|}, t\right)$ for the case of self-collisional Fokker-PlanckLandau term.

of $f$ in one point is distributed to all the velocity space points. Moreover, the solution conserves the initial value of the macroscopic quantities, as pictured in $4.2 \mathrm{a}$, and the parallel and perpendicular temperatures evolve till the isotropic equilibrium $T=T_{\perp}=T_{\|}$is reached, while $T$ is constant in time. Nevertheless, there is a relatively large variation in the species temperature, as seen in figure $4.2 \mathrm{~b}$. The error of $T$ respect to the initial value is in the order of $10^{-6}$, much higher than those found in one-dimensional conservative collision terms presented below. This results from the fact that fewer grid points on each direction have to be used to obtain a reasonable computational time in two-dimensional cases. In addition, the integration becomes more difficult as the domain employed accounts for the natural boundary condition at $v_{\perp} \rightarrow 0$. This hinders the numerical resolution of the problem, even when all the second order corrections presented in appendix B.3 are utilised. However, additional numerical analysis is required to further reduce this error. Nevertheless, the deviation is still small, and simulations are useful enough to compute transport coefficients, as it will be presented in section 5.3.

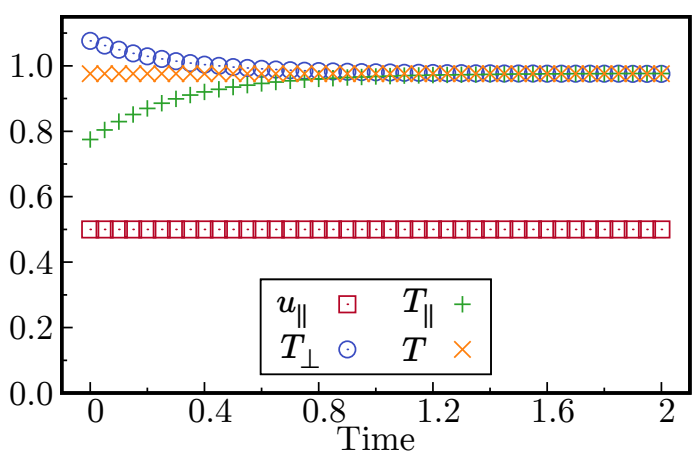

(a) Balance

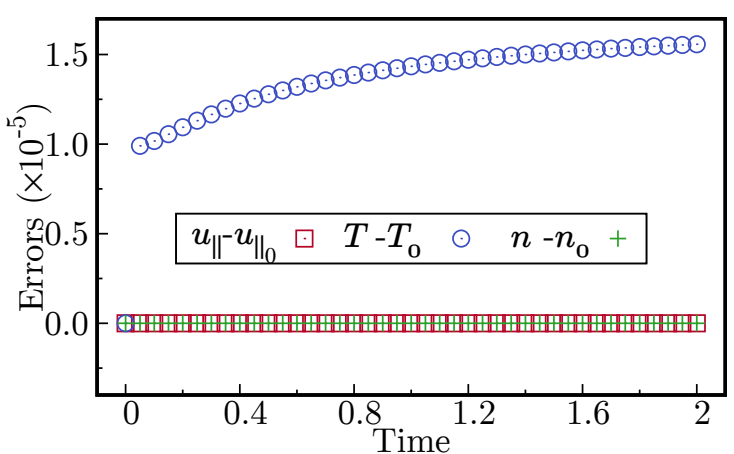

(b) Errors respect to initial condition

Figure 4.2: Balance (a) of macroscopic variables and errors respect to the initial condition (b) for the Fokker-Planck-Landau collisional term. 


\subsubsection{Hard Spheres (HS) potential case}

Multiple collision terms can be derived in the same way as the Fokker-Planck-Landau one by just modifying the kernels from (4.2). A particular interesting case arise for $V(\mathbf{r})=k r^{-4}$, for $n=4$, the so-called Maxwell's molecule potential [42,107,108]. This potential reduces the kernels of the convection diffusion parameters to

$$
d_{i}^{\gamma \gamma^{\prime}}=-\left(1+\frac{m_{\gamma}}{m_{\gamma^{\prime}}}\right) \mu^{\gamma \gamma^{\prime}} g_{i}, d_{i j}^{\gamma \gamma^{\prime}}=-\frac{\mu^{\gamma \gamma^{\prime}}}{2}\left(\delta_{i j} g^{2}-g_{i} g_{j}\right)
$$

where $\mu^{\gamma \gamma^{\prime}}$ is a collisional parameter. As we did with the Fokker-Planck-Landau operator, the case in a velocity space composed by the perpendicular and parallel directions to an electric field is studied. The resulting convective-diffusion coefficients from (4.1) are

$$
\begin{gathered}
D_{\perp}^{\gamma \gamma^{\prime}}=-\left(1+\frac{m_{\gamma}}{m_{\gamma^{\prime}}}\right) \mu^{\gamma \gamma^{\prime}} \int g_{\perp} f_{\gamma^{\prime}}^{\prime} \mathrm{d} \mathbf{v}^{\prime} ; D_{\|}^{\gamma \gamma^{\prime}}=-\left(1+\frac{m_{\gamma}}{m_{\gamma^{\prime}}}\right) \mu^{\gamma \gamma^{\prime}} \int g_{\|} f_{\gamma^{\prime}}^{\prime} \mathrm{d} \mathbf{v}^{\prime} ; \\
D_{\perp \perp}^{\gamma \gamma^{\prime}}=\frac{\mu^{\gamma \gamma^{\prime}}}{2} \int\left(g^{2}-g_{\perp} g_{\perp}\right) f_{\gamma^{\prime}}^{\prime} \mathrm{d} \mathbf{v}^{\prime} ; D_{\|\|}^{\gamma \gamma^{\prime}}=\frac{\mu^{\gamma \gamma^{\prime}}}{2} \int\left(g^{2}-g_{\|} g_{\|}\right) f_{\gamma^{\prime}}^{\prime} \mathrm{d} \mathbf{v}^{\prime} ; \\
D_{\phi \phi}^{\gamma \gamma^{\prime}}=\frac{\mu^{\gamma \gamma^{\prime}}}{2} \int\left(g^{2}-g_{\phi} g_{\phi}\right) f_{\gamma^{\prime}}^{\prime} \mathrm{d} \mathbf{v}^{\prime} ; D_{\perp \|}^{\gamma \gamma^{\prime}}=-\frac{\mu^{\gamma \gamma^{\prime}}}{2} \int g_{\perp} g_{\|} f_{\gamma^{\prime}}^{\prime} \mathrm{d} \mathbf{v}^{\prime} ;
\end{gathered}
$$

These coefficients can be expressed in their integral form to ease their numerical implementation

$$
\begin{aligned}
D_{\perp}^{\gamma \gamma^{\prime}}=-\left(1+\frac{m_{\gamma}}{m_{\gamma^{\prime}}}\right) \mu^{\gamma \gamma^{\prime}} n_{\gamma^{\prime}} v_{\perp} ; D_{\|}^{\gamma \gamma^{\prime}}=-\left(1+\frac{m_{\gamma}}{m_{\gamma^{\prime}}}\right) \mu^{\gamma \gamma^{\prime}} n_{\gamma^{\prime}}\left(v_{\|}-u_{\|}^{\gamma^{\prime}}\right) ; \\
D_{\perp \perp}^{\gamma \gamma^{\prime}}=\frac{\mu^{\gamma \gamma^{\prime}}}{2} n_{\gamma^{\prime}}\left(v_{\|}^{2}+E_{\|}^{\gamma^{\prime}}-2 v_{\|} u_{\|}^{\gamma^{\prime}}+E_{\perp}^{\gamma^{\prime}}\right) ; D_{\|\|}^{\gamma \gamma^{\prime}}=\frac{\mu^{\gamma \gamma^{\prime}}}{2} n_{\gamma^{\prime}}\left(v_{\perp}^{2}+2 E_{\perp}^{\gamma^{\prime}}\right) ; \quad(4.11) \\
D_{\phi \phi}^{\gamma \gamma^{\prime}}=\frac{\mu^{\gamma \gamma^{\prime}}}{2} n_{\gamma^{\prime}}\left(v_{\perp}^{2}+4 E_{\perp}^{\gamma^{\prime}}+v_{\|}^{2}+E_{\|}^{\gamma^{\prime}}-2 v_{\|} u_{\|}^{\gamma^{\prime}}\right) ; D_{\perp \|}^{\gamma \gamma^{\prime}}=-\frac{\mu^{\gamma \gamma^{\prime}}}{2} n_{\gamma^{\prime}}\left(v_{\perp} v_{\|}-v_{\perp} u_{\|}^{\gamma^{\prime}}\right),
\end{aligned}
$$

being

$$
n_{\gamma}=\int f_{\gamma} \mathrm{d} \mathbf{v}, u_{\|}^{\gamma}=\int v_{\|} f_{\gamma} \mathrm{d} \mathbf{v}, E_{\|}^{\gamma}=\int v_{\|}^{2} f_{\gamma} \mathrm{d} \mathbf{v}, E_{\perp}^{\gamma}=\int \frac{1}{2} v_{\perp}^{2} f_{\gamma} \mathrm{d} \mathbf{v},
$$

the norm, parallel velocity and anisotropic energy contributions per unit of mass in the parallel and perpendicular directions, respectively.

Conservation properties can be easily checked. With respect to the momentum conservation, as in the FPL situation, the relation $\left\langle m_{\gamma} D_{\|}^{\gamma \gamma^{\prime}}\right\rangle=-\left\langle m_{\gamma^{\prime}} D_{\|}^{\gamma^{\prime}}\right\rangle$ is verified if $\mu^{\gamma^{\prime} \gamma}=m_{\gamma}^{2} / m_{\gamma^{\prime}}^{2}$ is fulfilled, which also ensures the conservation of total energy.

The same piecewise initial function as the one employed in the case of the FPL operator, presented in equation (4.7) is used to numerically check the conservation properties. For this numerical case, $\mu^{\gamma \gamma}=1$. Figure 4.3 presents the evolution of the distribution function $f\left(v_{\perp}, v_{\|}, t\right)$. The abrupt initial condition relaxes to a Maxwellian distribution, 


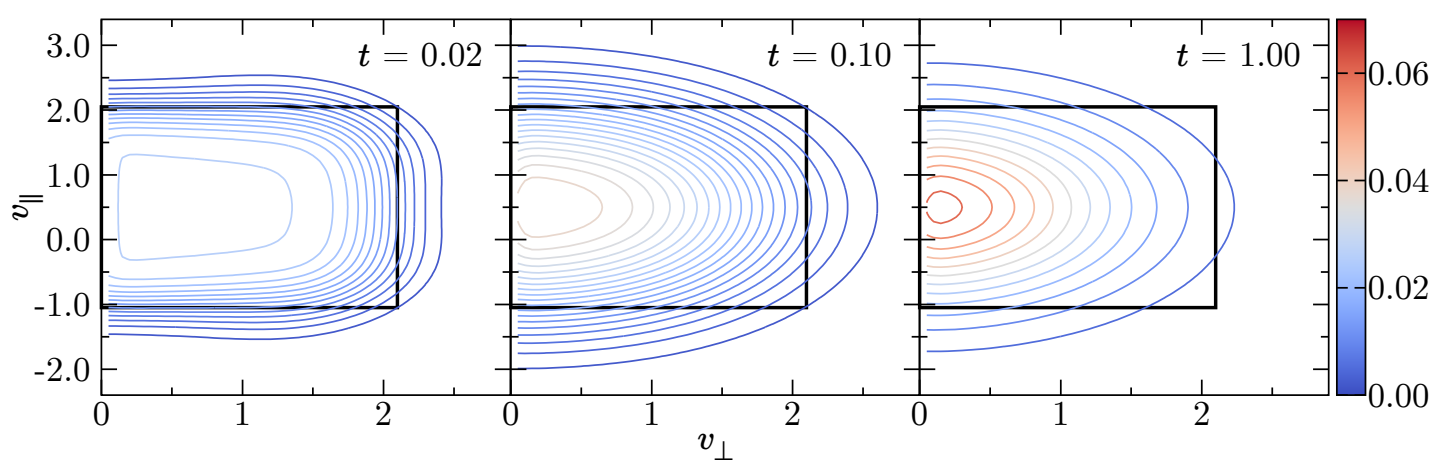

Figure 4.3: Distribution function $f\left(v_{\perp}, v_{\|}, t\right)$ for the case of self-collisional HS term.

and becomes smooth from the first iteration. As for the Fokker-Planck-Landau operator, the Maxwellian distribution arises naturally as a consequence of the drift and diffusive effects perfectly captured by the propagator. Moreover, parallel mean velocity is conserved as well as the temperature $T$. Isotropisation of $f$ arise when perpendicular and parallel temperatures evolve until the steady situation $T=T_{\perp}=T_{\|}$is reached, with a relaxation time lower than the FPL case.

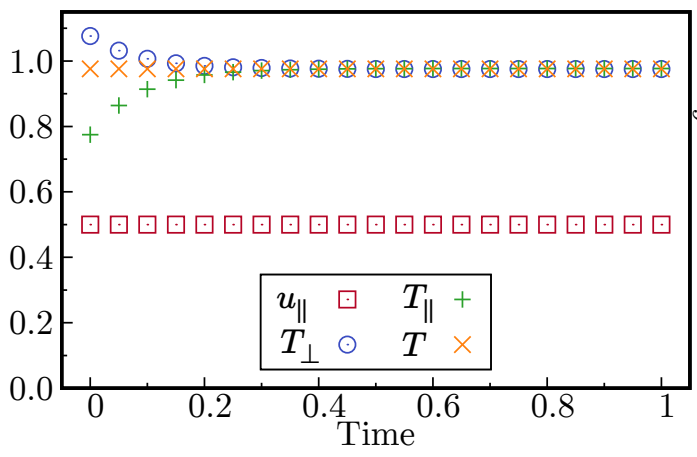

(a) Balance

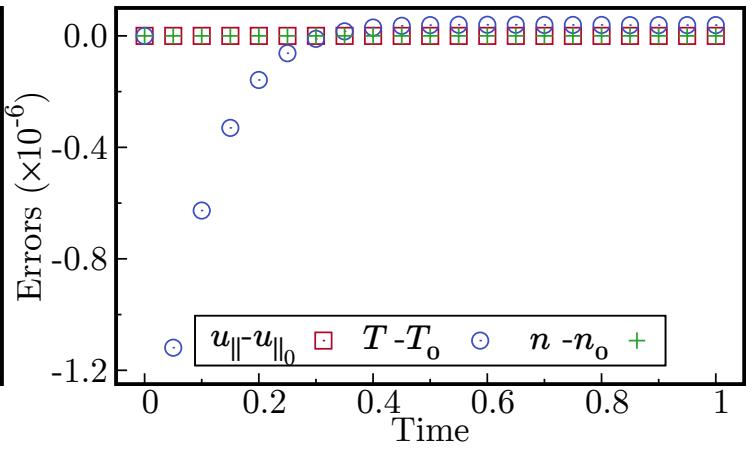

(b) Errors respect to initial condition

Figure 4.4: Balance (a) of macroscopic variables and errors respect to the initial condition (b) for the Hard Spheres collisional term.

\subsubsection{Dougherty collision term}

In several practical applications the Dougherty operator [91,109-111] has been used to describe non-lineal collisional terms, as we did in $[25,28,112]$ for the one-dimensional case. This two-dimensional form can be extracted from the previous Hard Spheres potential. A double integral over the diffusion coefficients $D_{i j}^{\gamma \gamma^{\prime}}$ is performed to obtain a Dougherty like collision operator- To keep the right physical dimensions, $D_{i j}^{\gamma \gamma^{\prime}}$ must 
be divided by $n_{\gamma}$. The resulting drift-diffusion terms take the form

$$
\begin{gathered}
D_{\perp}^{\gamma \gamma^{\prime}}=-\left(1+\frac{m_{\gamma}}{m_{\gamma^{\prime}}}\right) \mu^{\gamma \gamma^{\prime}} n_{\gamma^{\prime}} v_{\perp} ; D_{\|}^{\gamma \gamma^{\prime}}=-\left(1+\frac{m_{\gamma}}{m_{\gamma^{\prime}}}\right) \mu^{\gamma \gamma^{\prime}} n_{\gamma^{\prime}}\left(v_{\|}-u_{\|}^{\gamma^{\prime}}\right) ; \\
D_{\perp \perp}^{\gamma \gamma^{\prime}}=\frac{\mu^{\gamma \gamma^{\prime}}}{2} n_{\gamma^{\prime}}\left(E_{\|}^{\gamma}+E_{\|}^{\gamma^{\prime}}-2 u_{\|}^{\gamma} u_{\|} \gamma^{\prime}+E_{\perp}^{\gamma^{\prime}}\right) ; D_{\|\|}^{\gamma \gamma^{\prime}}=\frac{\mu^{\gamma \gamma^{\prime}}}{2} n_{\gamma^{\prime}}\left(2 E_{\perp}^{\gamma}+2 E_{\perp}^{\gamma^{\prime}}\right) ; \\
D_{\phi \phi}^{\gamma \gamma^{\prime}}=\frac{\mu^{\gamma \gamma^{\prime}}}{2} n_{\gamma^{\prime}}\left(2 E_{\perp}^{\gamma}+4 E_{\perp}^{\gamma^{\prime}}+E_{\|}^{\gamma}+E_{\|}^{\gamma^{\prime}}-2 u_{\|}^{\gamma} u_{\|}^{\gamma^{\prime}}\right) ; \\
D_{\perp \|}^{\gamma \gamma^{\prime}}=-\frac{\mu^{\gamma \gamma^{\prime}}}{2} n_{\gamma^{\prime}}\left(\sqrt{\frac{\pi E_{\perp}^{\gamma}}{2}} u_{\|}^{\gamma}-\left(\sqrt{\frac{\pi E_{\perp}^{\gamma}}{2}} u_{\|}^{\gamma^{\prime}}\right) ;\right.
\end{gathered}
$$

If the relation $\mu^{\gamma^{\prime} \gamma}=m_{\gamma}^{2} / m_{\gamma^{\prime}}^{2} \mu^{\gamma \gamma^{\prime}}$ is fulfilled, the conservation of energy and momentum is guaranteed, as in previous cases.

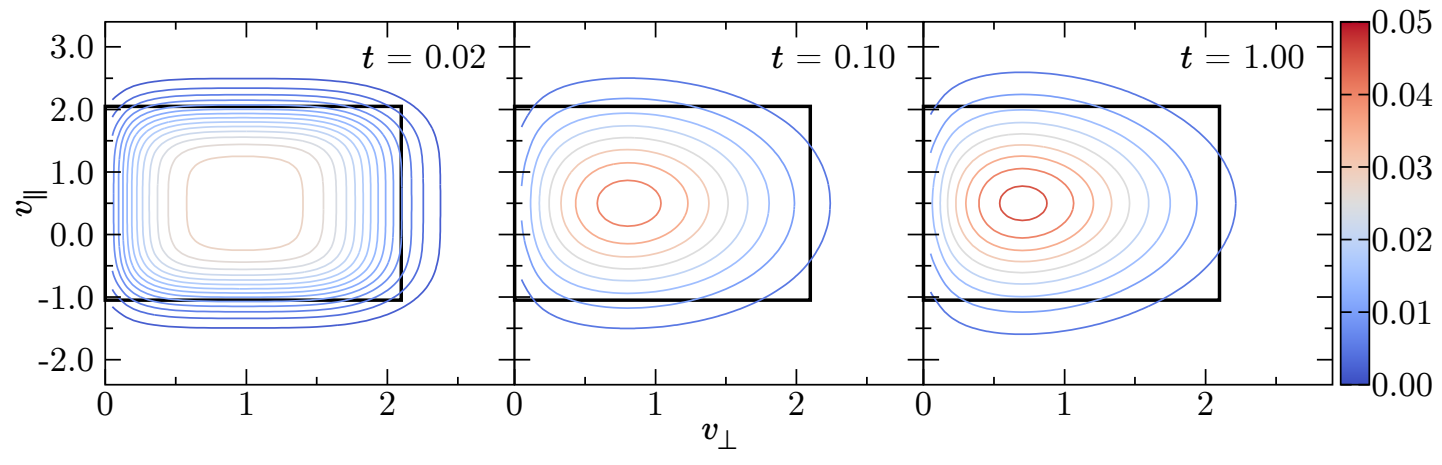

Figure 4.5: Distribution function $f\left(v_{\perp}, v_{\|}, t\right)$ for the case of self-collisions with a $2 \mathrm{~V}$ Dougherty term.

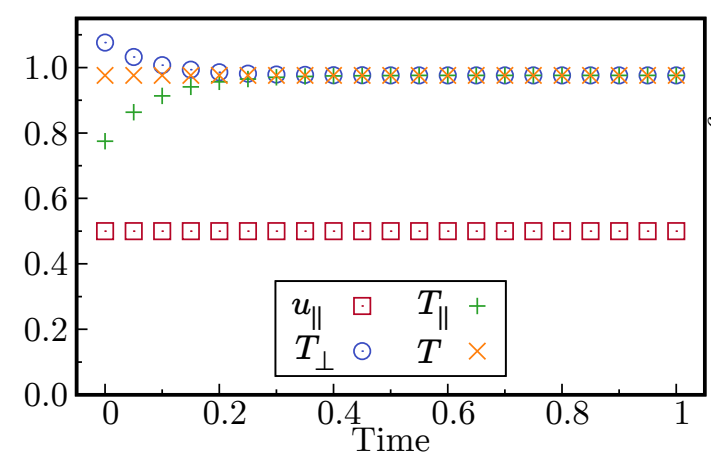

(a) Balance

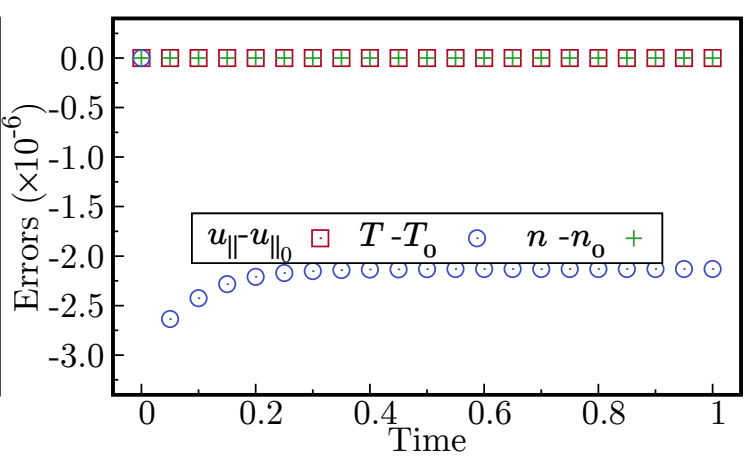

(b) Errors respect to initial condition

Figure 4.6: Balance (a) of macroscopic variables and errors respect to the initial condition (b) for the $2 \mathrm{~V}$ Dougherty collisional term.

Now, the conservative properties of the two-dimensional Dougherty operator are tested numerically with the PIM. As we did with previous operators, the function (4.7) is taken as an initial condition to test the method. Even though the evolution of the distribution function, figure 4.5, is smooth from the first time step and macroscopic quantities are conserved, figure 4.6, the steady situation deviate from a Maxwellian distribution function, although a higher entropy state than the initial one is reached 
when this term is employed. This results implies that the use of cylindrical geometry can induce spurious geometrical drift-diffusion terms and the direct derivation of Dougherty operator is not as straightforward as in Cartesian coordinates.

\subsubsection{One-dimensional Dougherty term}

The Dougherty collision term can be transposed into a $1 \mathrm{D}$ velocity space, $v \in(-\infty, \infty)$ without losing any of its conservation properties and the steady Maxwellian distribution. The multi-species drift-diffusion terms that represent this process can be derived from $[109,111]$ as

$$
D_{v}^{\gamma \gamma^{\prime}}=-\mu^{\gamma \gamma^{\prime}} \int\left(v-v^{\prime}\right) f_{\gamma^{\prime}}^{\prime} \mathrm{d} v^{\prime} ; D_{v v}^{\gamma \gamma^{\prime}}=\frac{\mu^{\gamma \gamma^{\prime}}}{2 n_{\gamma}} \iint \frac{\left(v^{\prime \prime}-v^{\prime}\right)^{2}}{2} f_{\gamma^{\prime}}^{\prime} f_{\gamma}^{\prime \prime} \mathrm{d} v^{\prime} \mathrm{d} v^{\prime \prime}
$$

These parameters can be expressed in a compact form, independently on the distribution function, as depending only on the velocity $v$ and mean velocity and energy, becoming

$$
D_{v}^{\gamma \gamma^{\prime}}=-\nu^{\gamma \gamma^{\prime}}\left(v-u_{\gamma^{\prime}}\right) ; D_{v v}^{\gamma \gamma^{\prime}}=\nu^{\gamma \gamma^{\prime}}\left(E_{\gamma}+E_{\gamma^{\prime}}-2 u_{\gamma} u_{\gamma^{\prime}}\right),
$$

where $\nu^{\gamma \gamma^{\prime}}=n_{\gamma^{\prime}} \mu^{\gamma \gamma^{\prime}}$ is the collision frequency characteristic of the momentum exchange rate between $\gamma$ and $\gamma^{\prime}$. This term results useful to analyse a short action potential interactions between particles, e.g., hard sphere collisions, when the evolution of the system is constrained to one direction. Moreover, it is easy to see how species $\gamma^{\prime}$ act as a viscous media for $\gamma$, as in a Brownian motion.

Following the same procedure presented above for two-dimensional terms, conservation of linear moment can be analytically achieved if $\left\langle m_{\gamma} D_{v}^{\gamma \gamma^{\prime}}\right\rangle=-\left\langle m_{\gamma^{\prime}} D_{v}^{\gamma^{\prime} \gamma}\right\rangle$, which is always true if $\mu^{\gamma^{\prime} \gamma}=\frac{m_{\gamma}}{m_{\gamma^{\prime}}} \mu^{\gamma \gamma^{\prime}}$. Even when this relation seems different from those derived above, they are equivalent as in the one-dimensional Dougherty term presented here the mass relation between both species $\left(1+m_{\gamma} / m_{\gamma^{\prime}}\right)$ is included in the collision parameter. In addition, energy conservation is fulfilled by the relation between collisional parameters above, meaning that $m_{\gamma}\left\langle D_{v v}^{\gamma^{\prime} \gamma}+v D_{v}^{\gamma^{\prime} \gamma}\right\rangle=-m_{\gamma^{\prime}}\left\langle D_{v v}^{\gamma^{\prime} \gamma}+v D_{v}^{\gamma^{\prime} \gamma}\right\rangle$ for the binary exchange. For the self-collisional case, as usual, conservation is fulfilled for any value of $\mu^{\gamma \gamma}$.

The numerical analysis of the Dougherty collision operators follows the same steps as in previous problems. The piece-wise continuous one-dimensional initial condition is

$$
f_{0}(q)=\frac{n_{0}}{2 \sqrt{3 T_{0} / m}}\left[H\left(v-u_{0}+\sqrt{3 T_{0} / m}\right)-H\left(v-u_{0}-\sqrt{3 T_{0} / m}\right)\right],
$$

where $n_{0}=1, u_{0}=0.5, T_{0}=1$ and $m=1$ are the initial density, velocity, temperature and mass, respectively; and $H(x)$ is the Heaviside function. For the 1D self-collisional case, we have

$$
D_{v}=n(v-u), D_{v v}=n\left(E-\frac{1}{2} u^{2}\right),
$$




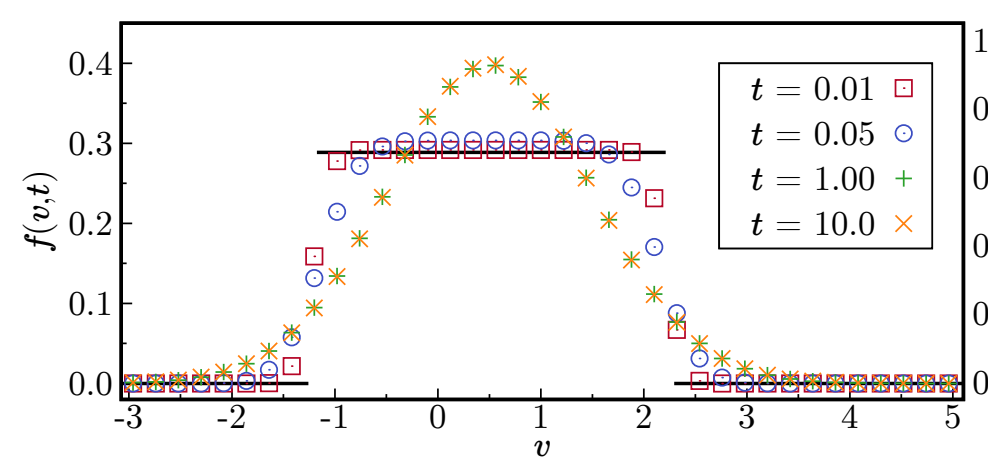

(a) Distribution function

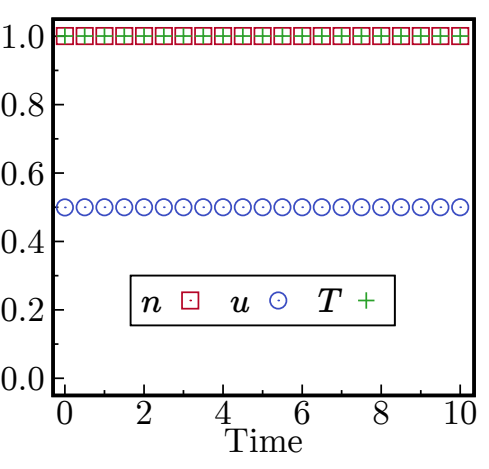

(b) Macroscopic moments

Figure 4.7: Evolution of the self-collisional Dougherty one-dimensional operator. Initial condition is pictured as a black solid line.

which is a non-dimensional form of (4.15), for $\mu=1$. Figure 4.7a shows how the discontinuous initial distribution function spreads and becomes continuous from the first time step. A Maxwellian distribution is rapidly reached, in a time of order $\sim 1$ keeping its form up to the end of the simulation.

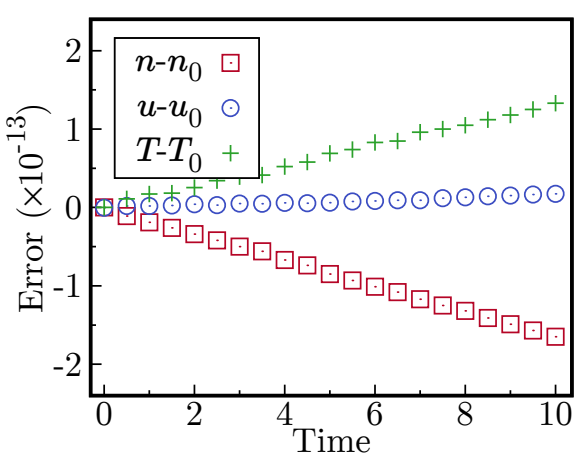

Figure 4.8: Numerical errors of the conserved quantities respect to initial condition for the one-dimensional Dougherty collision term.
The macroscopic moments, figure $4.7 \mathrm{~b}$ are always constant and no instabilities due to the discontinuous initial condition appear. Figure 4.8 presents the error of the macroscopic momentums respect to the initial condition. Small numerical errors appear and even when their value is orders of magnitude below the initial value $\left(\sim 10^{-13}\right)$, they should be accounted for in larger simulations, where the value of these errors may increase up to a level able to perturb the conservation of macroscopic quantities. However, these small errors, within the numerical fluctuations, do not perturb the evolution of this problem or the macroscopic values. Due to the numerical nature of the method, is impossible to have a free of error solution, but this case proves that the PIM produces a conservative solutions in all the scope of problems analysed in this dissertation.

It is expected that, conservation properties of the Dougherty collision term hold in the numerical description of the two species interaction if the relation between collision parameters presented above is fulfilled. In this numerical analysis, a mesh of length 50 for the species $a$ and 0.3 for the species $b$ is employed with 10001 points for each species, being $a$ and $b$ the generic ones in table 4.1. The time step is $\tau=10^{-2}$ and 500 iterations are calculated.

In figure 4.9, physical magnitudes of interest such as the mass fluxes and energy for each species and their sum are pictured. As said, total momentum and energy are 


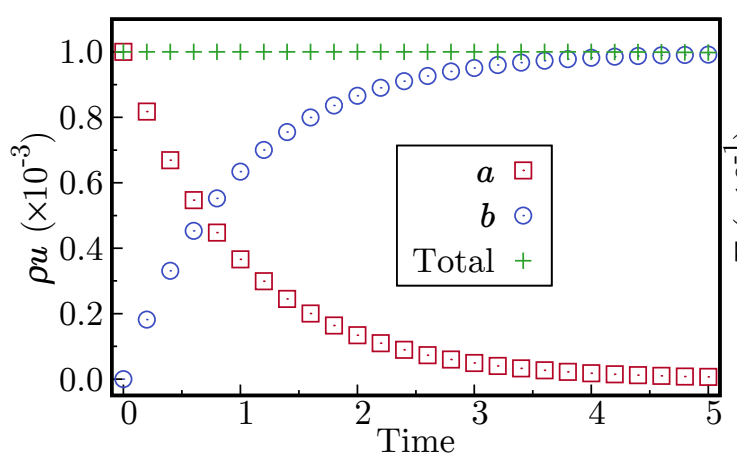

(a) Flux

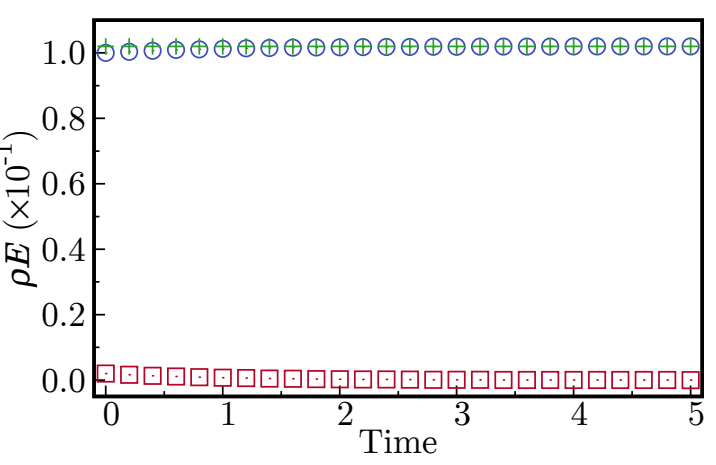

(b) Energy

Figure 4.9: Evolution of the conserved properties for the two-species collisional Dougherty operator. Values for each species and their sum are presented.

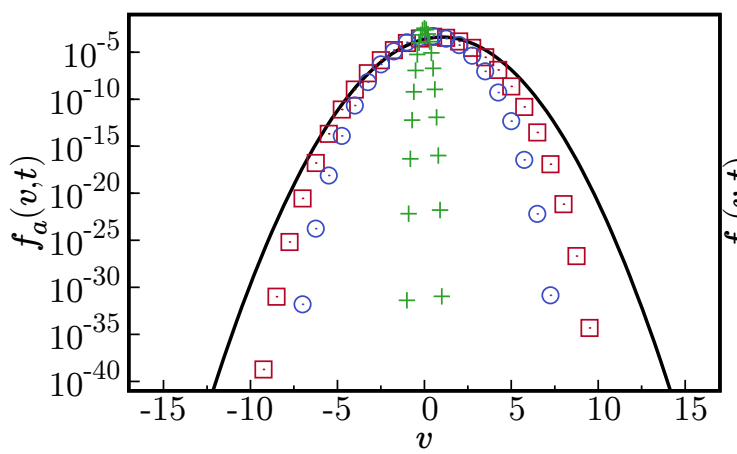

(a) $f_{a}$

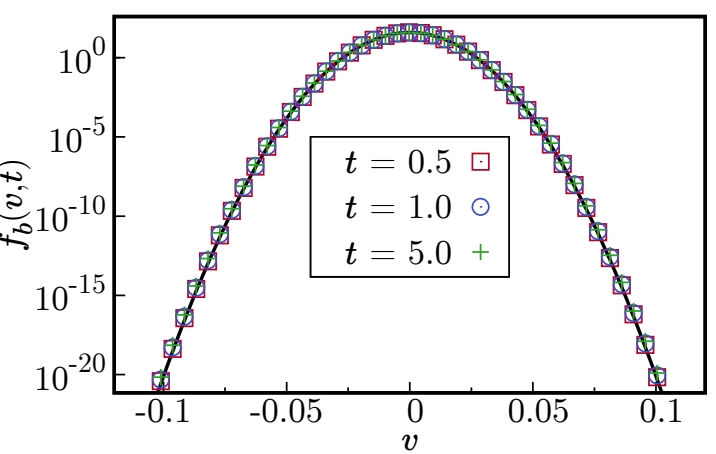

(b) $f_{b}$

Figure 4.10: Evolution of the distribution function for the two-species collisional Dougherty operator. Initial condition is represented with a black solid line.

numerically conserved, except for possible small errors. Mass fluxes evolve in completely different ways for each species. The heavy species is softly accelerated due to the sole effect of collisions and gains a small amount of energy. On the other hand, the light species is slowed down and its energy is diminished. The steady fluid velocities are equal for both species, but the huge difference in density and masses between them creates the gap in the mass flux presented in figure $4.9 \mathrm{a}$. In figure $4.9 \mathrm{~b}$, we can see how the light species $a$ loses part of its energy, which is acquired by the heavy species $b$, keeping the total value constant. This evolution is found only if both species are advanced simultaneously in time. If the heavy species is left fixed or if the collision terms are linearised, this dynamic could be modified, leading to losses of the total energy or different steady states. In that case, light particles would end up exchanging all of its energy with the fixed heavy species, resulting in a scenario were the total energy is lost. Therefore, for refined computations of involved non-linear problems, special care has to be taken to include physical effects that ensure conservation properties are fulfilled.

Here, very different dynamics are found for each species because of the disparate ratios used as initial conditions. In figure 4.10a, we can see a fast variation of $a$ species, which loses energy and momentum until it reaches a steady situation. On the other hand, 
$f_{b}$ seems to be unaltered during the simulation, and almost static with respect to its initial condition. Nevertheless, the analysis of macroscopic variables presented above shows that small but significant changes in the heavy species appears. This means that the study of slow species requires an accurate computation of its distribution function, in order to obtain the right macroscopic dynamics, because changes in the distribution function are small [24]. In terms of conservation and large scale analysis, the incorrect evolution of slow species may lead to inaccurate situations, specially in higher space and time scales.

\subsubsection{The 1D charge-charge term}

The evolution of a distribution function constrained to one direction under the effect of a Coulombian potential is still an open problem. When plasmas are approximately described as evolving in a one-dimensional velocity space, this view comes from considering there is a dominant cause, usually a strong external electric field, that makes the flow of particles move in one privileged direction, leaving the others unaltered. In this case, the scattering of charged particles may be constrained, limiting the interaction to the privileged direction of interest and sometimes leading to non-Maxwellian distribution functions $[86,88,113]$. Here, an approach to describe this dynamics based on the full Fokker-Planck-Landau operator is taken

This operator describing the one-dimensional motion constraint was firstly presented in [25] by myself and others and a review and more extensive analysis is performed now. Here, we set the motion equation of a one-dimensional velocity distribution function, without considering it as a marginal distribution obtained after integration on perpendicular velocity.

Here, the charge-charge collision term is constructed for the one velocity dimensional case by taking as a reference the complete well-known Landau collisional operator [19, $58,78]$ in a spatially homogeneous plasma. A physical realisation of this model could be related, for instance, to the description of a plasma between two planar infinite metal walls, where an intense electric field appears between the plates [85,114]. In such a system, the planar geometry leads to plasma species distribution functions depending only on the velocity component $v$, parallel to the direction perpendicular to both walls, lying in the privileged direction established by the electric field. Since a test particle of mass $m_{\gamma}$ and velocity $v$ is scattered by particles of velocity $v^{\prime}$ with distribution function $f_{\gamma^{\prime}}\left(v^{\prime}, t\right)$, a small change of the particle velocity $\Delta v$ in the preferred direction may be considered as a result of an average Coulombian interaction force among charges. Thence, the dynamical frictional effect experienced by $m_{\gamma}$ can be phenomenologically modelled as an effective force of uniform intensity opposite to the relative velocity $v-v^{\prime}$. This contribution can be understood as a Coulomb's like law for dry friction, a case also studied in the theoretical frame of Brownian motion in [61]. The cumulative effect of many interactions gives rise to the drift coefficient $D_{v}^{\gamma \gamma^{\prime}}$ which is related to the change 
of the expectation value of $\Delta v$ per unit of time $(\langle\Delta v\rangle / \Delta t)$ as

$$
D_{v}^{\gamma \gamma^{\prime}}=\frac{\langle\Delta v\rangle}{\Delta t}=-\mu^{\gamma \gamma^{\prime}}\left(1+\frac{m_{\gamma}}{m_{\gamma^{\prime}}}\right) \int \operatorname{sgn}\left(v-v^{\prime}\right) f_{\gamma^{\prime}}^{\prime} \mathrm{d} v^{\prime} .
$$

Here, $\operatorname{sgn}(\cdot)$ is the sign function with $\operatorname{sgn}(0)=0$ and $\mu_{\gamma \gamma^{\prime}}$ is the collisional parameter related to plasma properties as mass and charge. Now, following similar steps presented in [78] to derive a FPL operator, we argue to obtain a non-linear self-consistent diffusion coefficient. Beside the friction force, we assume that the test charge is subjected to random fluctuating forces of stochastic nature, responsible of the $f_{\gamma}$ spreading in velocity space. This diffusive behaviour is computed by the coefficient $D_{v v}^{\gamma \gamma^{\prime}}$, related to the average value of $(\Delta v)^{2} / 2$ per unit of time $\left(\left\langle(\Delta v)^{2}\right\rangle / 2 \Delta t\right)$. Assuming that $\Delta v$ is of order $v-v^{\prime}$ while $\Delta v / \Delta t$ is proportional to the friction term $\operatorname{sgn}\left(v-v^{\prime}\right)$, a diffusion coefficient proportional to the average value of $\left(v-v^{\prime}\right) \operatorname{sgn}\left(v-v^{\prime}\right)=\left|v-v^{\prime}\right|$ is proposed

$$
D_{v v}^{\gamma \gamma^{\prime}}=\frac{\left\langle(\Delta v)^{2}\right\rangle}{2 \Delta t}=\mu^{\gamma \gamma^{\prime}} \int\left|v-v^{\prime}\right| f_{\gamma^{\prime}}^{\prime} \mathrm{d} v^{\prime}
$$

assuming that $\int|v| f_{\gamma} d v$ remains finite.

With this selection of drift-diffusion coefficients the resulting one-dimensional collisional term for charge-charge interaction behaves as the complete Fokker-Planck-Landau operator, in fact, it can be easily checked that it provides a well posed conservative collision operator for a one dimensional plasma, since $\mu^{\gamma \gamma^{\prime}}$ does not alter the conservation properties of the operator, as long as the relation $\mu^{\gamma^{\prime} \gamma}=\frac{m_{\gamma}^{2}}{m_{\gamma^{\prime}}^{2}} \mu^{\gamma \gamma^{\prime}}$ is fulfilled. This relation, as previously mentioned, ensures the conservation of total momentum and energy in the collisional process.. Moreover, similar differential relations for the drift and diffusion coefficients fulfilled by the complete Fokker-Planck-Landau operator are also satisfied. In particular, using of the relation $\partial_{v} \operatorname{sgn}\left(v-v^{\prime}\right)=2 \delta\left(v-v^{\prime}\right)$ the properties

$$
\begin{aligned}
D_{v}^{\gamma \gamma^{\prime}} & =-\left(1+\frac{m_{\gamma}}{m_{\gamma^{\prime}}}\right) \frac{\partial}{\partial v} D_{v v}^{\gamma \gamma^{\prime}} \\
\frac{\partial}{\partial v} D_{v}^{\gamma \gamma^{\prime}} & =-2 \mu^{\gamma \gamma^{\prime}}\left(1+\frac{m_{\gamma}}{m_{\gamma^{\prime}}}\right) f_{\gamma^{\prime}},
\end{aligned}
$$

hold. These are equivalent to the properties held by the divergences of the diffusion tensor and the drift vector for the Fokker-Planck-Landau operator [43,106], where the distribution function is also obtained by the derivative of the drift coefficient.

At this stage, the self-collisional case is numerically analysed. The distribution function from (4.16) is employed as an initial condition with 30001 points, a grid of length 300 centred at $v=0$ and $\tau=10^{-2}$. This long grid is required to allow the distribution's tails, which follow a power decaying law, reach a value small enough to not affect the conservation of macroscopic quantities. These tails behaviours have been experimentally measured in solar wind plasmas, which seem to evolve in a one-dimensional velocity space [86]. Figure 4.11 depicts the time evolution of macroscopic moments and the distribution function. First important result is the non-Maxwellian steady state 


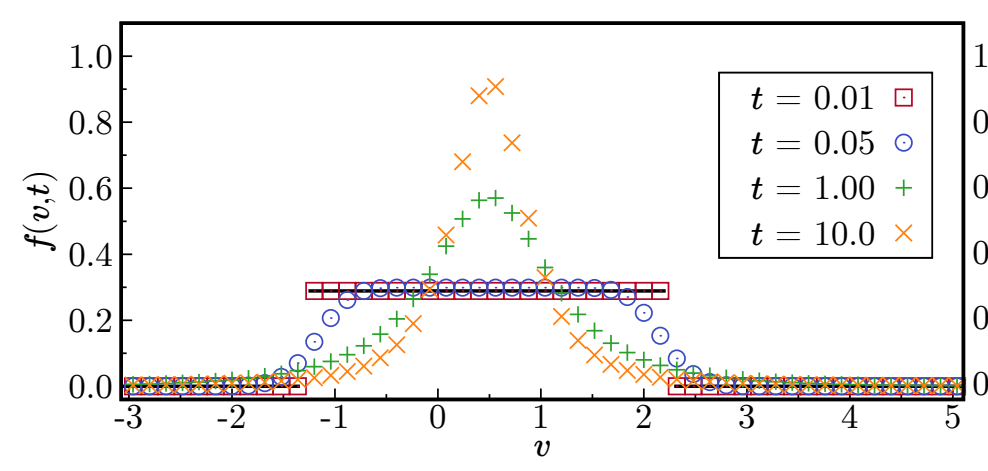

(a) Distribution function

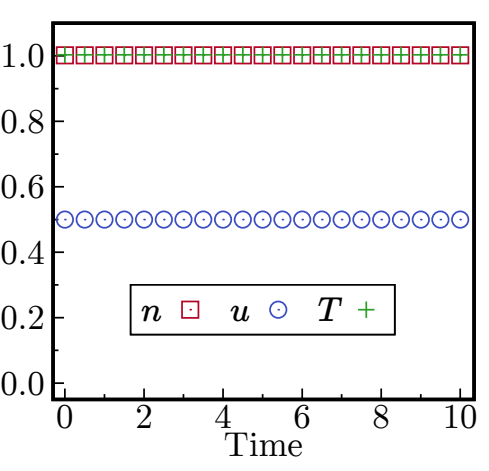

(b) Macroscopic moments

Figure 4.11: Evolution of the self-collisional one-dimensional charge-charge operator. Initial quantities are conserved in every step of the simulation. The discontinuous initial function (black line) end up relaxing into the reference function.

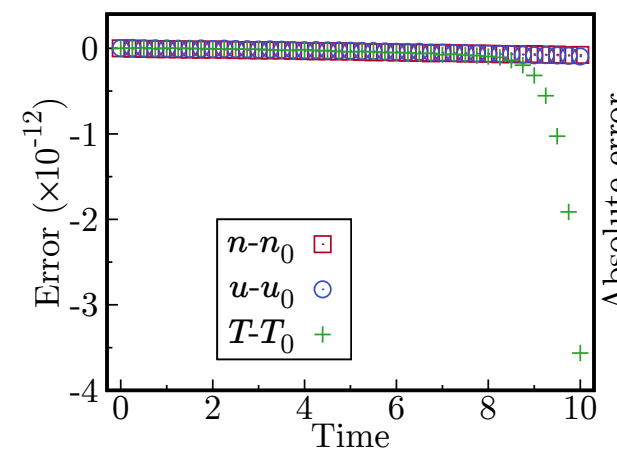

(a) Errors of momentums

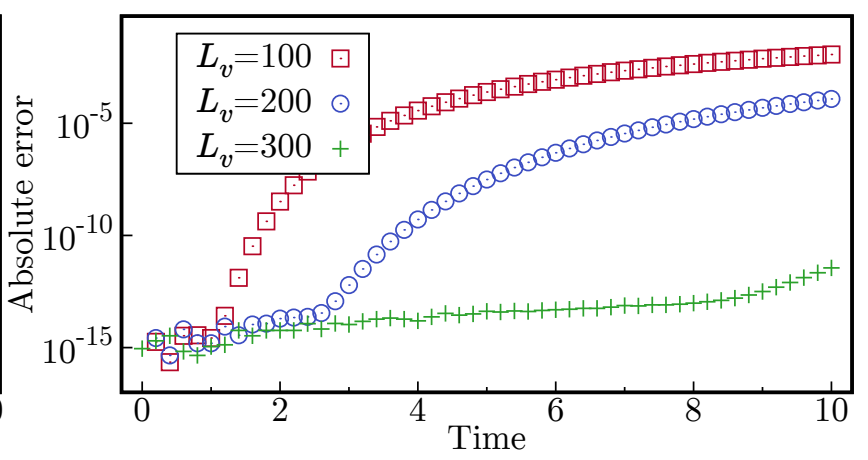

(b) Influence of grid length length

Figure 4.12: (a) Numerical errors of the conserved quantities respect to the initial condition for the one-dimensional charge-charge collision term. (b) Influence in the energy conservation for different grid lengths. Due to the power law decaying tails, long grids are required.

reached, which means that entropy in not maximised. This can be explained due to the one-directional constraint assumed in the derivation of this term. Nevertheless, the moments of the distribution function are constants, regardless its final shape or evolution which means that the operator is conservative.

Small errors appears in the calculation of momentum and temperature evolutions respect to the initial condition, figure 4.12a. Moreover, temperature error starts to increase negatively for large simulation time due to the finiteness of the numerical grid. Relatively large values of $f$ reach the end of the numerical domain, meaning that distribution tails are not properly represented. Due to the power law decay of the distribution's tails, conservation of macroscopic quantities, specially energy, can be violated in the numerical problem if values high enough appear at the end of the grid, although still the solution behaves as analytical one in the inner points. This is more evident for the computation of total energy since $\frac{1}{2} f v^{2}$ may be high at the grid edges. To reduce this issue, larger grids can be used, but this increases the computational time and resources required to solve the problem. In figure $4.12 \mathrm{~b}$, the error of the 


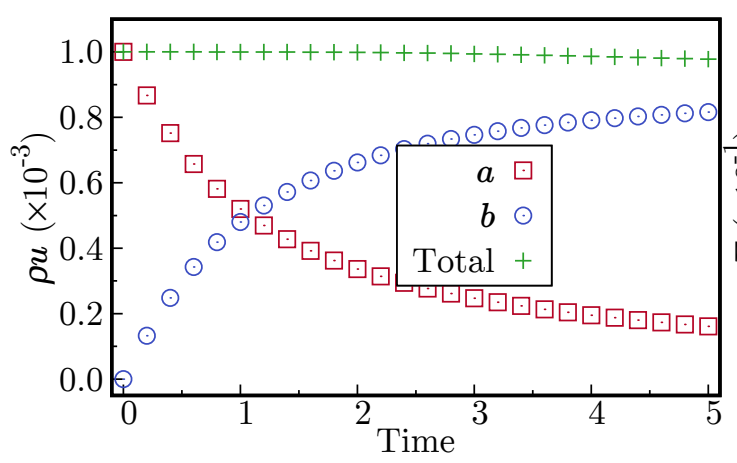

(a) Flux

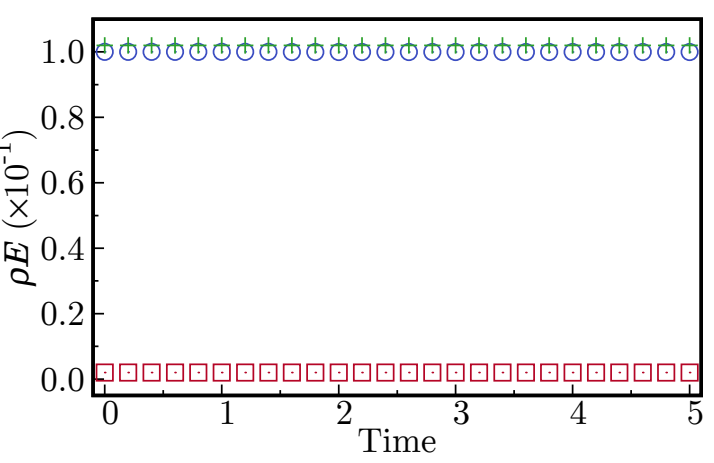

(b) Energy

Figure 4.13: Evolution of the conserved properties for the two-species one-dimensional chargecharge collision operator. Values for each species and their sum are presented.

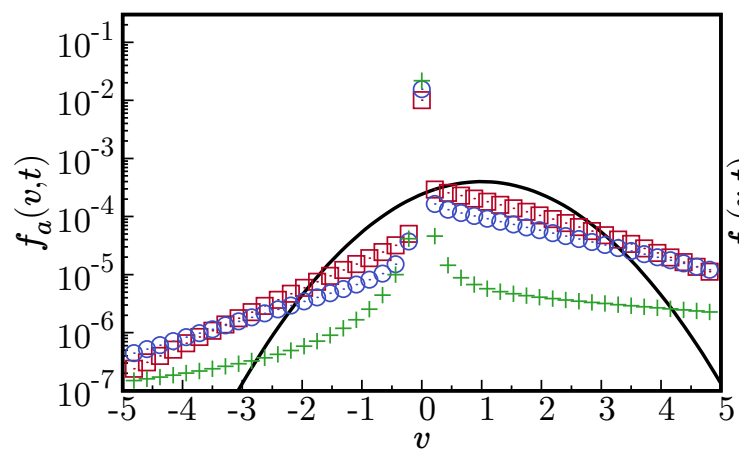

(a) $f_{a}$

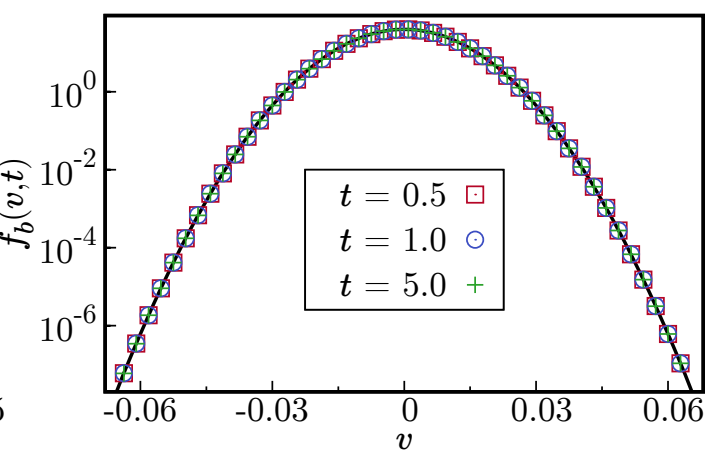

(b) $f_{b}$

Figure 4.14: Evolution of the distribution function for the two-species collisional onedimensional charge-charge operator. Initial condition is represented with a black solid line.

temperature respect to the initial value for different grid lengths is presented. It is easily checked that for shorter meshes the temperature error increases after just a few iterations, affecting the conservation properties.

Conservation properties considerations also hold in the two species interaction problem. To numerical analyse this case, grid lengths of 280 and 0.15 dimensionless units are employed for species $a$ and $b$ respectively with 32001 points for each species. In figure 4.13, the momentum and energy flows for both species and their sum are presented. Differences in the relaxation times with respect to the previous Dougherty operator can be found, even when macroscopic variables are the same at the end of the simulation. First, mass fluxes of both species take a larger time to cross each other and the small variations in the energy found in figure $4.9 \mathrm{~b}$ do not seem to appear here. The collision term is also responsible of energy exchanges, but its rate is lower than for other operators, as Dougherty. This lower exchange rate appears also in the self-collisional Fokker-Planck-Landau presented in section 4.1.1, where the time required to reach a quasi-steady state is twice to HS or Dougherty cases.

For the distribution function for both species, appearing in figure 4.14, the heavy one 
$b$ is almost static, whereas $a$ changes drastically reaching a steady state with a peak of particles around $v=0$ with a power law decaying tails, quite different from it initial Maxwellian shape.

Here, it is important to remark that the PIM captures non-Maxwellian distribution functions and detects relaxation times properly, even when propagators employed are Gaussian-like.

\subsubsection{The Bhatnagar, Gross, and Krook (BGK)}

A simplification of Boltzmann collision term was presented by Bhatnagar, Gross, and Krook in [80] for small amplitude process in one component gases and extended later for a binary interactions in [81] for plasmas and mixtures of gases. This popular and widely used approximate model is the basic of many in kinetic theoretical models for plasma. In this section, we explore the numerical and analytical evolution of this operator, where its more general form

$$
\left(\frac{\partial f_{\gamma}}{\partial t}\right)_{\gamma \gamma^{\prime}}=-\sum_{\gamma^{\prime}} \nu^{\gamma \gamma^{\prime}}\left(f_{\gamma}-\bar{f}_{\gamma}\right)
$$

is adopted, being $\bar{f}_{\gamma}$ a Maxwellian function of the same density of species $\gamma$ but velocity and temperature of species $\gamma^{\prime}$ and $\nu^{\gamma \gamma^{\prime}}$ is the collision frequency. In an absence of external forces or other non-homogeneous terms, the time rate of change of macroscopic quantities for the species $\gamma$ becomes

$$
\begin{aligned}
\dot{n}_{\gamma} & =0 \\
n \dot{\mathbf{u}}_{\gamma} & =-\nu^{\gamma \gamma^{\prime}}\left(n_{\gamma} \mathbf{u}_{\gamma}-n_{\gamma} \mathbf{u}_{\gamma^{\prime}}\right) \\
n \dot{E}_{\gamma} & =-\nu^{\gamma \gamma^{\prime}}\left(n_{\gamma} E_{\gamma}-n_{\gamma} E_{\gamma^{\prime}}\right) .
\end{aligned}
$$

If the summation for the mass flux of species is performed, the relation

$$
\sum_{i=\gamma, \gamma^{\prime}} m_{i} n_{i} \dot{\mathbf{u}}_{i}=-\left(\nu^{\gamma \gamma^{\prime}} m_{\gamma} n_{\gamma}-\nu^{\gamma^{\prime} \gamma} m_{\gamma^{\prime}} n_{\gamma^{\prime}}\right)\left(\mathbf{u}_{\gamma}-\mathbf{u}_{\gamma^{\prime}}\right)
$$

appears, which is zero for any $\gamma, \gamma^{\prime}$ if $\nu^{\gamma^{\prime} \gamma}=\nu^{\gamma \gamma^{\prime}} m_{\gamma} m_{\gamma^{\prime}}^{-1} n_{\gamma} n_{\gamma^{\prime}}^{-1}$ is fulfilled, also ensuring the conservation of total energy. Of course, if $\gamma^{\prime}=\gamma$, no change in the macroscopic momentums appears independently of the $\nu^{\gamma \gamma}$ value.

This conservative operator has some issues regarding discontinuous or sharp distribution functions that may appear in the system evolution. Distribution functions are usually smooth and continuous, but it is common to represent heavy mass low temperature species (as ions and neutrals in weakly ionized plasmas) or beam of particles as Dirac deltas or as histograms. This can perturb the effect that this operator produces on the distribution function, specially for the first steps of the evolution. 


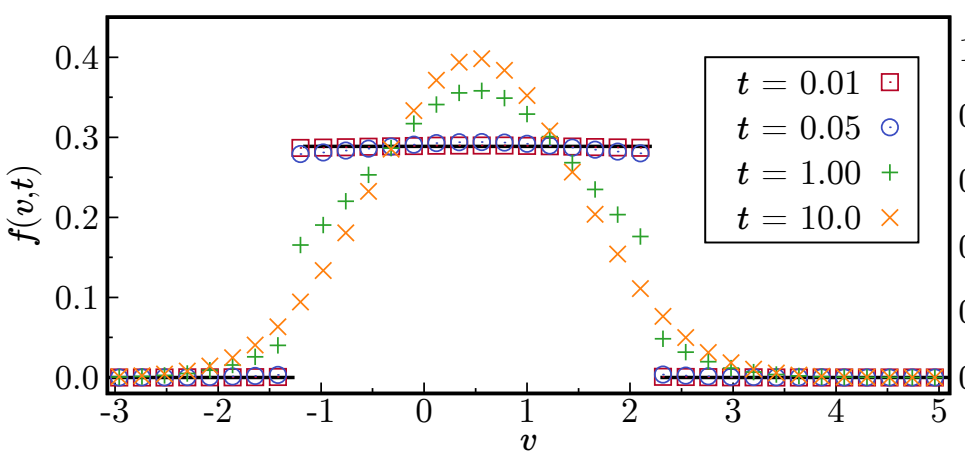

(a) Distribution function

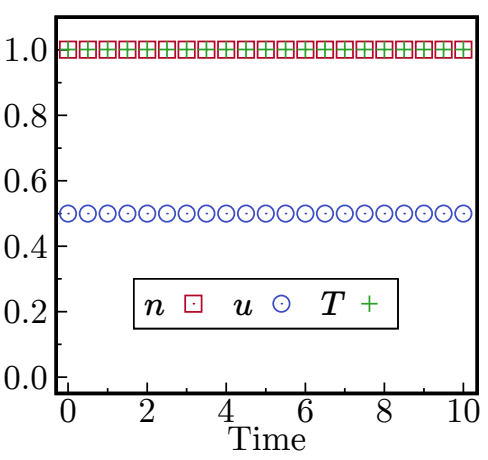

(b) Macroscopic moments

Figure 4.15: Evolution of the self-collisional BGK operator. Initial condition is pictured as a black solid line.

We explore up to what extend a natural discontinuous evolution with BGK has a physically meaning solution, being

$$
\frac{\partial f}{\partial t}=-\nu(f-\bar{f})
$$

the problem to solve, where $\nu$ is a collision frequency set to 1 in this non-dimensional problem, and $\bar{f}$ is a Maxwellian distribution with the same density, mean velocity and temperature as the time evolving solution. In the numerical self-consistent problem, the distribution function from (4.16) is employed as initial condition with 10001 points, a grid of length 20 and $\tau=10^{-2}$. The distribution function of the previous time step and its numerically obtained macroscopic variables are employed to calculate the non-homogeneous term, including $\bar{f}$. However, the reference function could be constructed taking the initial macroscopic values as they are conserved quantities in this specific problem. Although, updating $\bar{f}$ at each time step provides a self-consistent problem that allows to check if small numerical errors that inevitably appear have an impact in the conservation properties. This more general test-case accounts for variations in the reference values, as it can happen by inter-

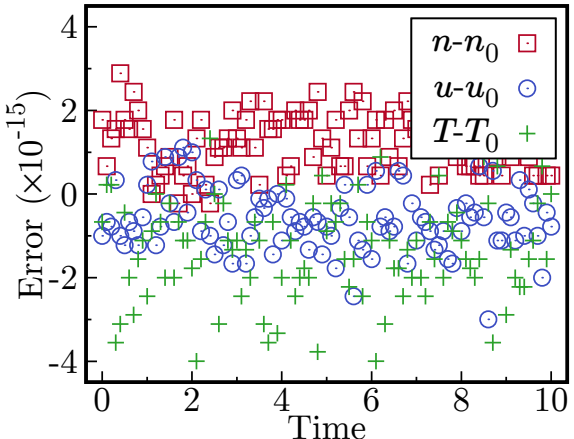

Figure 4.16: Numerical errors of the conserved quantities respect to initial condition for BGK collision term. actions with other species, non-homogeneous terms, boundary conditions or external forces.

The balance of moments and $f$ for the self-collisional case are presented in figure 4.15. The discontinuous initial distribution function (black line) evolves, as expected, towards a Maxwellian one but the discontinuities still hold in its evolution. This means that the BGK operator does not produce a continuous distribution function as a drift-diffusive operator does, independently of the number of points employed in the simulation. Nevertheless, this discontinuity does not affect the stability of the numerical problem 


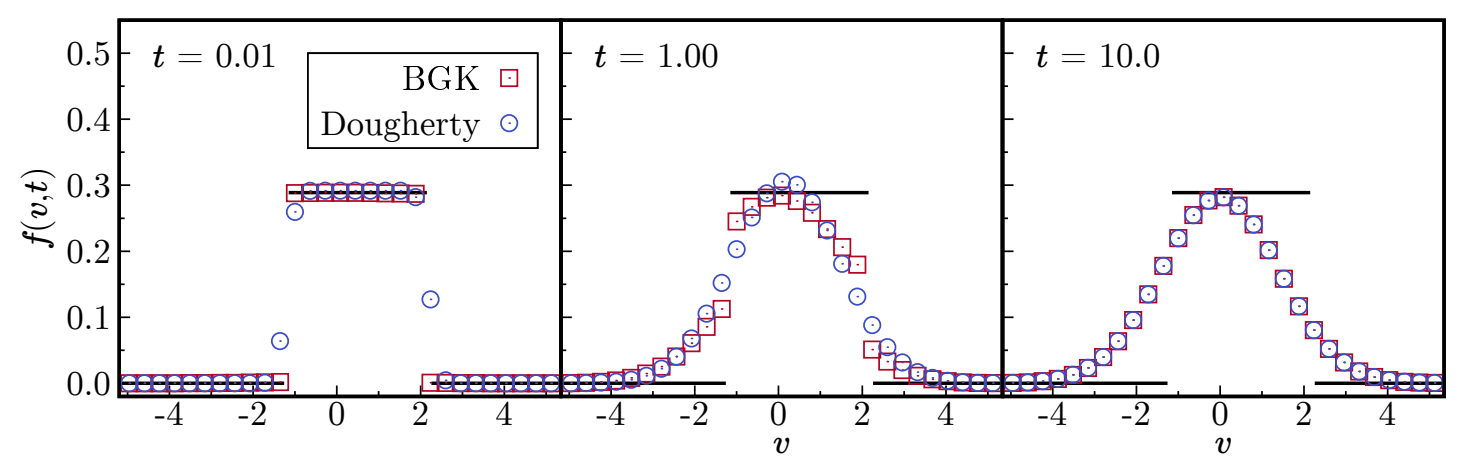

Figure 4.17: Evolution of the distribution function for two different collision terms, BGK and Dougherty, starting from an abrupt initial condition and relaxing into a Maxwellian of $T=2$.
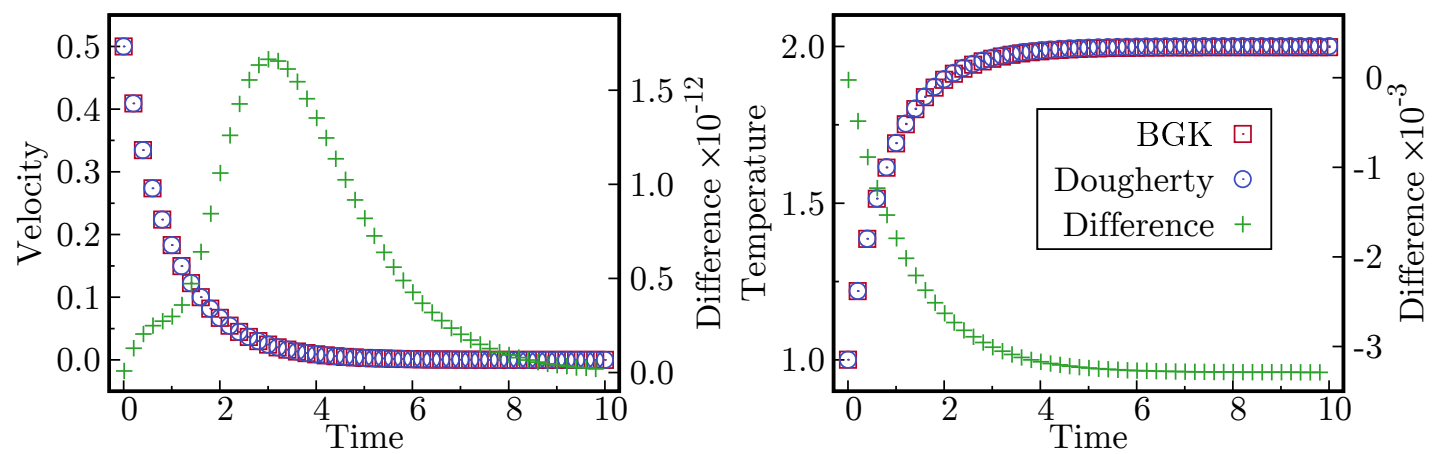

Figure 4.18: Evolution of the macroscopic moments for two different collision terms and its difference (green arrows).

and the macroscopic quantities values whose errors, shown in figure 4.16, are in the order of $10^{-15}$.

A relevant analysis to fully understand the evolution produced by BGK is the comparison with Dougherty term, by analysing typical relaxation times. Initial condition is the one employed to analyse the self-collisional cases. Taking the reference state as a Maxwellian distribution function with $u=0$ and $T=2$ in dimensionless units, the collision terms behave now as if the test particles collided with a fix background of the same mass and density, but at different temperature and mean velocity. For the BGK term, the reference function $\bar{f}$ must be constructed according to the new steady state, while that for the Dougherty term, the macroscopic variables $u_{\gamma^{\prime}}$ and $E_{\gamma^{\prime}}$ must be set to the appropriate values.

Significant differences appear between both solutions, figure 4.17. First, the difference evolution has a smooth and continuous shape from the first time step, while the BGK one presents discontinuities that hold even after a large number of iterations. This differentiation is important because drift-diffusive collision terms (as Fokker-PlanckLandau or Dougherty's) always produce a smooth solution, closer to the steady state. Therefore, BGK can lead to $f$ with discontinuities that has no physical meaning. This is due to diffusion acts as a global phenomena, while the production of source-sink terms, as BGK, is local in $v$ space. Nevertheless, both operators provide similar evolutions of 


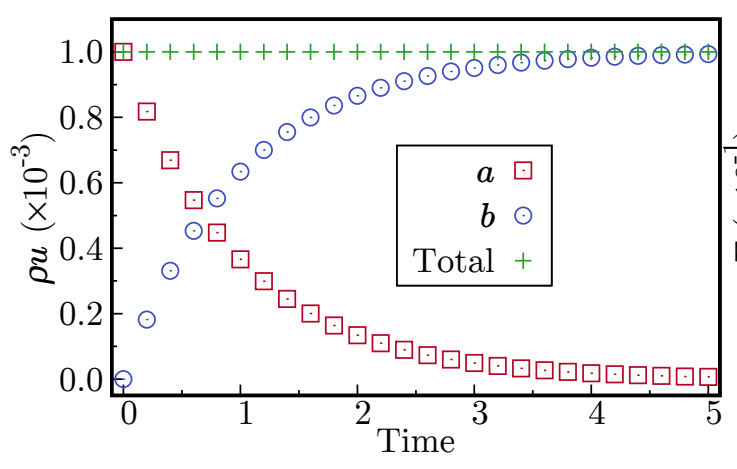

(a) Flux

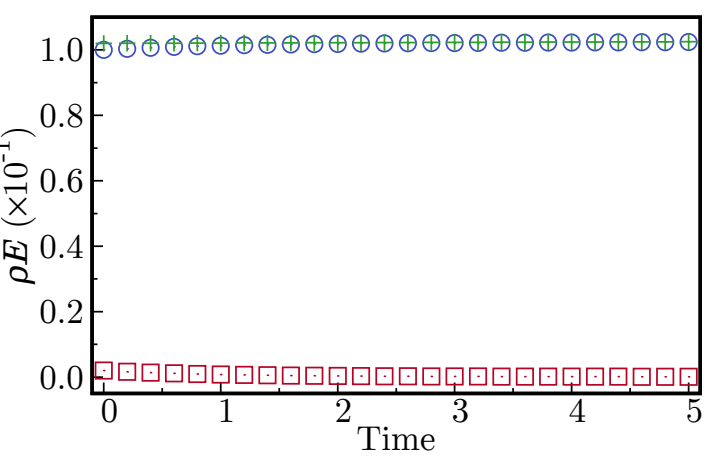

(b) Energy

Figure 4.19: Evolution of the conserved properties for the two-species collisional BGK operator. Values for each species and their sum are presented.

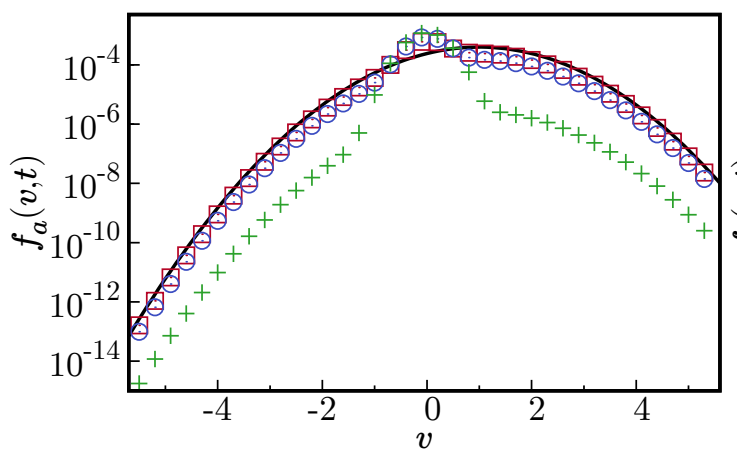

(a) $f_{a}$

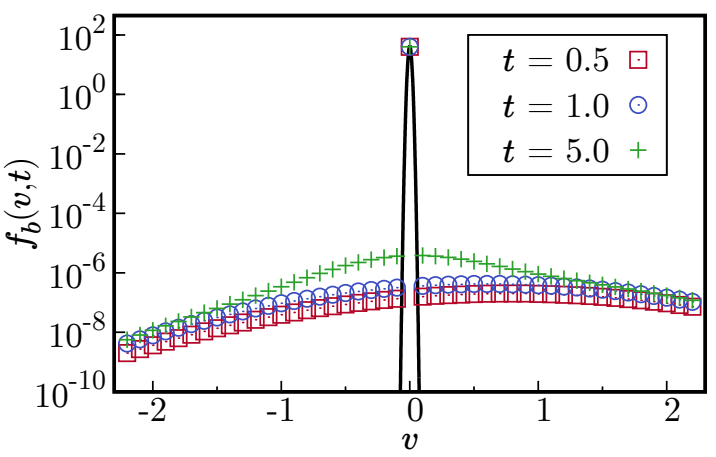

(b) $f_{b}$

Figure 4.20: Evolution of the distribution function for the two-species collisional BGK operator. Initial condition is represented with a black solid line.

the $f$ moments, figure 4.18. Surprisingly, both solutions take the same time to reach the steady state at $u=0$ and $T=2$, but a relative large difference appears in the temperature, with deviations of order of $10^{-3}$, due to the different numerical nature of both operators.

Continuing with our reliability analysis of the BGK term, the case of two-species interaction is examined. Conservative properties for the BGK collision term are also maintained if the relation between collision frequencies stated above holds. For a mesh of length 50 with 10001 points for each species and time step is $\tau=10^{-2}, 500$ iterations are calculated. The similar grid in both cases reduces the numerical implementation, as non-homogeneous term has a local effect over $f_{a}$. If different grids for each species are employed, some information is lost because non-homogeneous terms extends outside the numerical domain. This can become an important problem if collisions between disparate species are present. The mass flux and total energy are pictured in figure 4.19 showing very similar evolutions to the one-dimensional Dougherty operator although, again, important differences in the distribution functions profiles, figure 4.20, arise.

A clear effect is established: due to the local effect of the operator, BGK terms only 
influence those points where the distribution function is different from the reference state. This means that not all points may feel the collisions and distribution function may reach odd situations, as in figure 4.20 , where both distribution functions are depicted. The time evolving and steady functions are not smooth. For the species $a$, a deformation appears in the velocity space where distribution function profiles of species $b$ has a non-zero value. On the other hand, tails of the species $b$ are quickly energised, distorting $f_{b}$ close $v=0$. As it happened in the one-dimensional case, the evolution of macroscopic quantities in a BGK collision operator almost coincide with Dougherty's ones, but the distribution function evolves quite different, far from being a Maxwellian, but but with relaxation evaluations different fro each $v$. Nevertheless, it is worth mentioning that if self-collisions where included, smother distributions functions could be reached, as they tend to relax the distribution function into the reference state $\bar{f}$.

This non-continuous solution results in an entropy evolution not maximised at each time step. These significant differences in $f$ evolution strictly means that the collision operator selected for a problem can lead to distributions functions that distort results for higher moments of $f$.

\subsubsection{Entropy evolution considerations}

The entropy of a system is a relevant quantity to test the evolution towards a equilibrium state on an isolated system. Its calculation in kinetic models results arduous since its requires precise computation of the complete distribution function. Usually, a system tends to reach a steady of maximum equilibrium, but this is not true, for example, if an external action drives the system in some way such the entropy decreases, the system is not isolated and the external action constraints its evolution. All collision terms studied in this chapter, except for the charge-charge one-dimensional model, are entropically coherent, reaching a higher entropy steady state, which is usually the Maxwellian distribution. An important question to answer is if all collision operators increase Boltzmann entropy at the same rate, because this provides information about physically reliable the distribution function evolution towards an equilibrium is, regardless if the macroscopic variables are conserved. The analysis of the system entropy provides fundamental information on how self-collision terms relax the distribution function, specially from anisotropic initial situations, to the steady state. The main result of this analysis is found in figure 4.21, where the entropy evolution for the self-collisional cases is presented. Entropy is defined in non-dimensional units in its Boltzmann form

$$
s=-\int f \log f \mathrm{~d} \mathbf{v} .
$$

It can be seen how the collision operators relax the species distribution function to a Maxwellian, but time evolution do not coincide among them [19]. For the twodimensional terms, similar steady states appear, but in the Dougherty one, entropy is slightly smaller. In addition, Fokker - Planck-Landau operator has a lower relaxation time if compared with the other two cases. For the one-dimensional cases, the BGK 


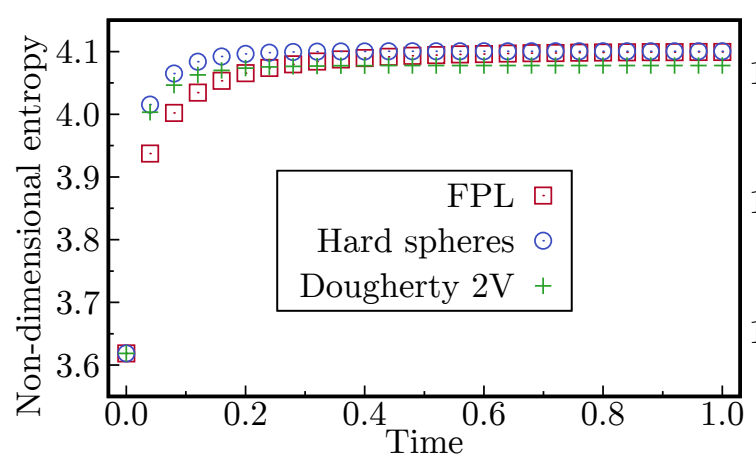

(a) Two dimensional terms

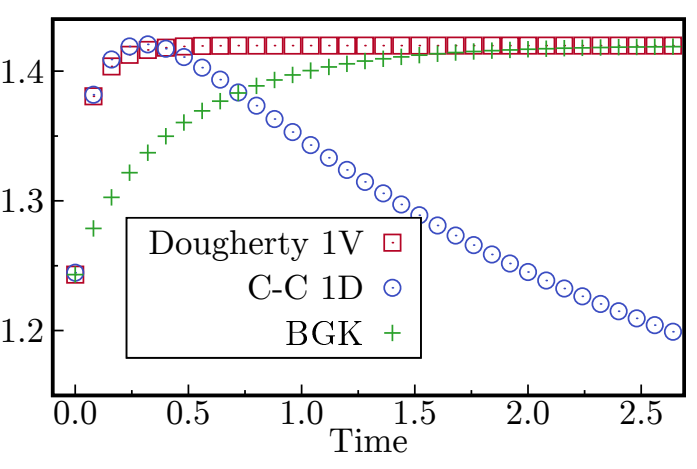

(b) One dimensional terms

Figure 4.21: Boltzmann entropy evolution for the self-collision terms studied in this chapter.

term results in an entropy evolution at a lower rate than the Dougherty's term, as a consequence of the discontinuous evolution of $f$. Dougherty operator relaxes the initial sharp function in a short time, $\sim 1$, while BGK takes up to 2 time units to reach the steady state. This evolution is also coherent with the fact that collisions are required to reach a thermodynamic equilibrium and that the time required to reach it is highly influenced by the collision operators utilised to describe the species exchanges.

\subsection{Influence of self-collisions in the evolution of discon- tinuous $f$ moments}

In some situations, plasmas or neutral gases may evolve from abrupt initial or boundary conditions or may generate thin structures, as layers or sheaths $[18,115-117]$, that make difficult the numerical resolution and may lead to non-physical evolutions or inaccurate solutions. For instance, collimated charges beam or localised emissive walls, as well as the electric applied field may result in an abrupt plasma evolution. These situations may also arise when two plasmas, of different characteristics, interpenetrate each other, giving rise to spatial diffusion and a new plasma mixture of the initial ones. In light of previous discussions, we can assume that self-collisions are the main cause to isotropisation and thermalisation in the two regions, from a microscopic point of view.

This fact motives the analysis of the collisional effects on the evolution of a one species neutral gas with discontinuous initial macroscopic variables. To isolate this effects, no electromagnetic force or interaction with other species is assumed. The initial condition has different macroscopic variables for $x \leq 0$ and for $x>0$ implying in practice that three zones arise at any time $t>0$ : a left and right side with the initial unperturbed properties and a central zone that connects both areas. Three scenarios are analysed: a non-collisional case, BGK and Dougherty models for collisional effects. The noncollisional case follows the Vlasov equation in an absence of external forces as

$$
\frac{\partial f}{\partial t}+v \frac{\partial f}{\partial x}=0
$$




\begin{tabular}{cccc}
\hline & $n$ & $u$ & $T$ \\
\hline$x \leq 0$ & 1 & 1 & 1 \\
$x>0$ & 0.1 & 0 & 1 \\
\hline
\end{tabular}

Table 4.2: Initial macroscopic values that generate a time evolving shock.

This means $f(x, v, t)$ evolves as being constant along the characteristic curve $x-v t$, as $f(x, v, t)=f_{0}(x-v t, v)$ where $f_{0}$ is the initial distribution function. Nevertheless, characteristics curves disappear for both collisional cases. These examples enclose a double purpose: first, analyse the influence of collisions when initial macroscopic discontinuous variables are employed and second, study the possible differences due to different collision terms in macro and microscopic views. The values of $n, u$ and $T$ on each initial region are given in table 4.2. The distribution function is Maxwellian in both zones. With these initial conditions, a front of high density and velocity at $x \leq 0$ appears, that mixes with the front on the right side for $t>0$. The collisional cases evolve with a collision frequency of $\mu=10$ to overstate the effect of collisions. In this scenario the convective motion in the $x-v$ space is responsible of the system evolution in all cases, whereas the relaxation of $f$ depends on the collisional model.

Evolution of the macroscopic velocity for this problem is pictured at three time frames in figure 4.22. Even when the three solutions are quite similar, collisions tend to reduce the mean velocity. The self-collisions do not drastically influence the mean velocity since it is conserved, as we have seen in the previous sections, and differences may be caused by the particular numerical implementations or by gradients in the system evolution.

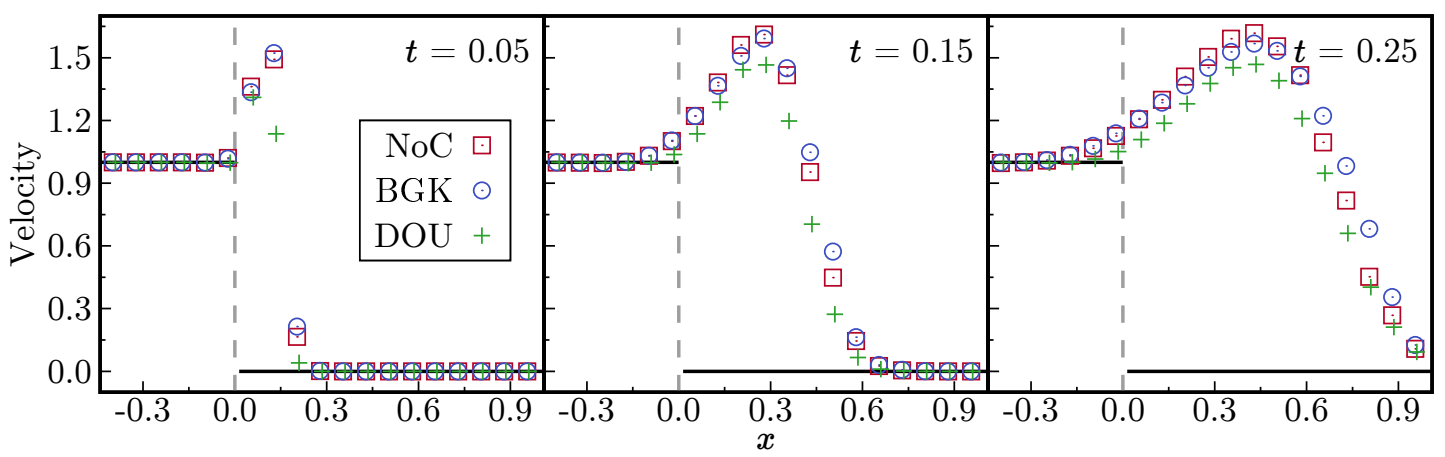

Figure 4.22: Velocity evolution after the discontinuous initial condition. Values at $t=0$ are represented with a black solid line.

If we analyse the distribution function contours obtained for the three models, figure 4.23 , notable differences among them emerge. In figure $4.23 \mathrm{a}$ we can see how the initial discontinuity at $x=0$ holds for the non-collisional case and just evolves following the convective motion related with $v$. As seen in figure 4.22, this convective evolution makes macroscopic variables change because particles of different velocities are entering into the new regions, i.e., particles with negative velocity component in $x>0$, at $t=0$, 


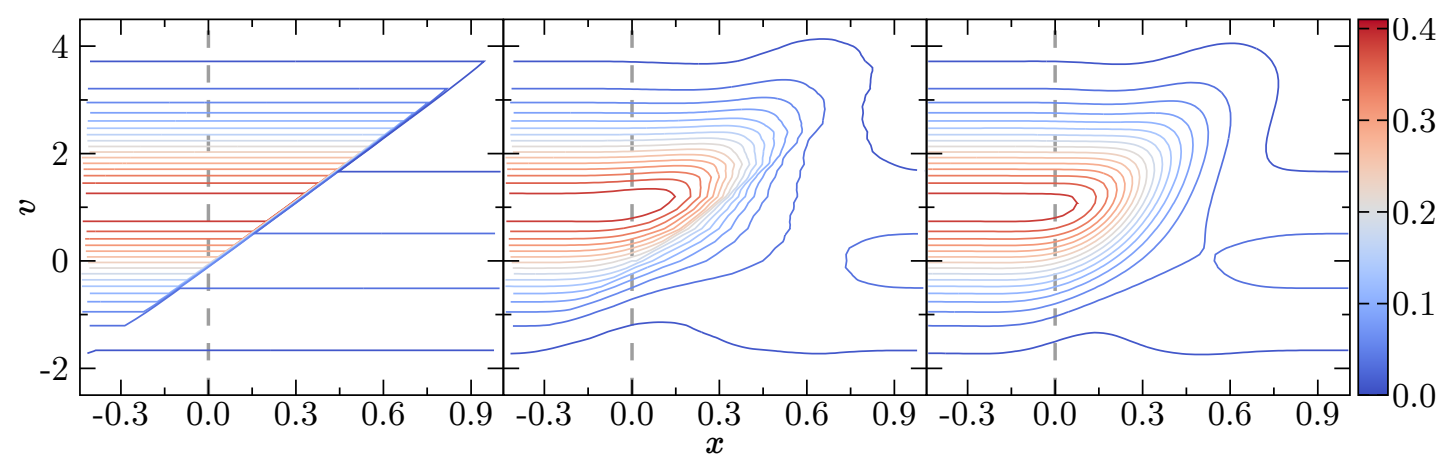

(a) Non-collisional

(b) $B G K$

(c) Dougherty

Figure 4.23: Distribution function at $t=0.25$ for the non-collisional and the two collisional cases. Dashed line represent the initial position of the discontinuity.

pass to $x \leq 0$ zone, and vice-versa, for $t>0$. If collisions are neglected, no relaxation appears and a discontinuous distribution is function held at any time. Three regions can be clearly identified in figure 4.23a. Close to $x<-0.3$, the distribution function at the right hand side has not penetrated the initial condition as it remains constant. The same happens for $x>0.9$, where the distribution function remains constant. However, in the intermediate area, a mixture of both zones exists and the moments vary at each position.

When collisions are accounted for, the discontinuity in the distribution function disappears giving a smoother evolution of $f(x, v, t)$. However, for BGK collisions, figure $4.23 \mathrm{~b}$, a discontinuous shape holds and the solution is not as smooth as in the Dougherty case, figure $4.23 \mathrm{c}$. In fact, two characteristic curves would solve the problem of BGK collisions, and the initial $f_{0}(x, v)$ along these characteristics retains the discontinuities in the solution. The division of the three zones stated above becomes less clear when collisions are included, yet it can be identified. In the case of Dougherty collisions, discontinuity has disappear completely due to collisional effects and a smooth solution is found in all the domain, and the effects of constant $f$ along the characteristic disappears. Thence, it is expected that Dougherty collisional case has a higher entropy, as distribution function is closer to the Maxwellian one in all the domain. Besides the differences in both collisional cases similar profiles of the distribution function and analogous zones can be identified.

In synthesis, collisions highly affect the shape of the distribution function, leading it close to a Maxwellian in all the domain, with negligible effect on the two first order moments. Nevertheless, the shape of the distribution function influence the entropy growth, which is coherent with the fact that collision are required to reach a thermodynamic equilibrium. The BGK operator shows a situation where a sharp $f$ appears in the system evolution as a consequence of the initial discontinuity, while the Dougherty case produces a smooth distribution function in all the domain from the first iteration, closer to a more realistic evolution. 


\subsection{Charge-exchange}

The widely considered resonant charge-exchange phenomenon $[118,119]$ describes the effect of a positive ion interacting with a neutral particle with the capture of one of its electrons. The exchange of energy may be considered small and the original ion, that has becomes a neutral particle after the exchange, retains almost all its energy, while the new ion moves at the velocity of the former neutral. The reaction process is expressed as

$$
A^{+}+A^{0} \stackrel{\sigma^{e x}}{\longrightarrow} A^{0}+A^{+},
$$

where the superscript represents the charge of the particles and $A$ represents a generic particle or molecule. In this process, the total charge and particle densities are conserved, unlike in ionization or recombination processes. This process is most likely to influence the system dynamics when energetic ions appears, e.g., those ions that have been accelerated by intense electric field interact with slow neutral particles [120,121].

Charge-exchange terms are usually modelled, from a kinetic point of view, by a Boltzmann like collision term [121], as

$$
\begin{aligned}
& s_{e x}^{i}(\mathbf{v})=\int \sigma^{e x}\left[f_{0}\left|\mathbf{v}-\mathbf{v}^{\prime}\right| f_{i}^{\prime}-f_{i}\left|\mathbf{v}-\mathbf{v}^{\prime}\right| f_{0}^{\prime}\right] \mathrm{d} \mathbf{v}^{\prime}, \\
& s_{e x}^{0}(\mathbf{v})=\int \sigma^{e x}\left[f_{i}\left|\mathbf{v}-\mathbf{v}^{\prime}\right| f_{0}^{\prime}-f_{0}\left|\mathbf{v}-\mathbf{v}^{\prime}\right| f_{i}^{\prime}\right] \mathrm{d} \mathbf{v}^{\prime},
\end{aligned}
$$

where 0 refers to neutral particles, $i$ to ions, primed functions means that they are evaluated in the primes velocity $\mathbf{v}^{\prime}$ and $\sigma^{e x}$ is the cross-section for the charge-exchange effective collision. As done with the elastic collision terms, we study now the conservation properties of the term presented here from analytical and numerical points of view. Let us start by assuming that the ions and neutrals have a Dirac delta distribution function as

$$
f_{i}=\delta\left(\mathbf{v}-\mathbf{u}_{i}\right) ; f_{0}=\delta(\mathbf{v}),
$$

where $\mathbf{u}_{i}$ is the ions mean velocity, higher than the neutral one to represent energised ions free of external force or other effects and constant cross-section. With this in mind, equations (4.31) and (4.32) become

$$
\begin{aligned}
& s_{e x}^{i}(\mathbf{v})=\sigma^{e x}\left[f_{0}\left|\mathbf{v}-\mathbf{u}_{i}\right|-f_{i}|\mathbf{v}|\right] \\
& s_{e x}^{0}(\mathbf{v})=\sigma^{e x}\left[f_{i}|\mathbf{v}|-f_{0}\left|\mathbf{v}-\mathbf{u}_{i}\right|\right] .
\end{aligned}
$$

The changes in macroscopic moments for both species can be obtained by integrating these expressions in the velocity space, resulting in

$$
\begin{aligned}
& \dot{n}_{i}=\dot{n}_{0}=0, \\
& n \dot{\mathbf{u}_{i}}=-\dot{n} u_{0}=-\sigma^{e x} \mathbf{u}_{i} u_{i}, \\
& \dot{n E_{i}}=-\dot{n} E_{0}=-\frac{\sigma^{e x}}{2} u_{i}^{3} \text {, }
\end{aligned}
$$


It can be easily checked how the total energy and momentum remain constant in this model for charge-exchange process. Also, this conservation holds for any distribution function, because any $f$ can be expressed as an infinite sum of Dirac's deltas. This analysis can be easily extended for a general $\sigma^{e x}$ that depends on the particles difference velocity $\left|\mathbf{v}-\mathbf{v}^{\prime}\right|$ as in [121]. Moreover, the change in macroscopic quantities is more intense as $\left|\mathbf{u}_{i}\right|$ increases.

Conservation properties stated above and evolution of both species are numerically studied now. To do so, two species (ions and neutrals) of different densities, temperatures and velocities are considered. In this numerical example, both species have a mass of 1 dimensionless units, but it is important to remark that single charged ions and neutral masses differ in one electron mass. A density ratio of 0.001 between ions and neutrals is chosen. In addition, a small difference in the initial temperature of both species is assumed: $T_{0}=1$ and $T_{i}=1.1$. The main difference between both species relies on the ions initial mean velocity $u_{i}=5$, while neutrals are assumed initially at rest.

On the other hand, ion-neutral elastic collision is modelled as a Dougherty term. Selfcollisions are neglected because they do not affect the momentum and energy exchange we are interesting on. In the test-case presented in this section, the cross section of the charge-exchange process takes different values as $0,0.1$ and 1 , while elastic collisions have $\mu^{i 0}=1$. A case without ion-neutral elastic collision but with charge-exchange is performed. Usually in low energy plasmas, charge-exchange order of magnitude is lower than the charge-neutral elastic collision term, since ions are not energised. With the range of values used for $\sigma^{e x}$ the influence of this term in the species dynamics is analysed. An external force of $10^{-8}$ is also applied, equivalent to an electric field $\sim 0.1 \mathrm{~V} / \mathrm{m}$. Firstly, let us rewrite the term presented in (4.31) and (4.32) as the onedimensional operator

$$
\begin{aligned}
& s_{e x}^{i}(v)=\int \sigma^{e x}\left[f_{0}\left|v-v^{\prime}\right| f_{i}^{\prime}-f_{i}\left|v-v^{\prime}\right| f_{0}^{\prime}\right] \mathrm{d} v^{\prime}, \\
& s_{e x}^{0}(v)=\int \sigma^{e x}\left[f_{i}\left|v-v^{\prime}\right| f_{0}^{\prime}-f_{0}\left|v-v^{\prime}\right| f_{i}^{\prime}\right] \mathrm{d} v^{\prime} .
\end{aligned}
$$

The integration of these terms is performed numerically at each time step by a simple rectangle method, using the distribution functions of the previous iteration. Figure 4.24 presents the evolution of the macroscopic velocities for neutrals and ions. Chargeexchange interaction acts as a new collision term, relaxing the macroscopic variables and making the system to require less time to reach a quasi-steady situation ${ }^{1}$. The higher the cross section is, the higher the exchange between species. Even when the same steady state is reached in all cases, the time required to achieve it varies depending on the cross-section values and the exchange terms accounted for.

The temperatures of both species are shown in figure 4.25. Neutral temperature increases monotonically until it reaches a maximum value, relatively higher than the

${ }^{1}$ The small electric field prevents the system to reach a steady state. 


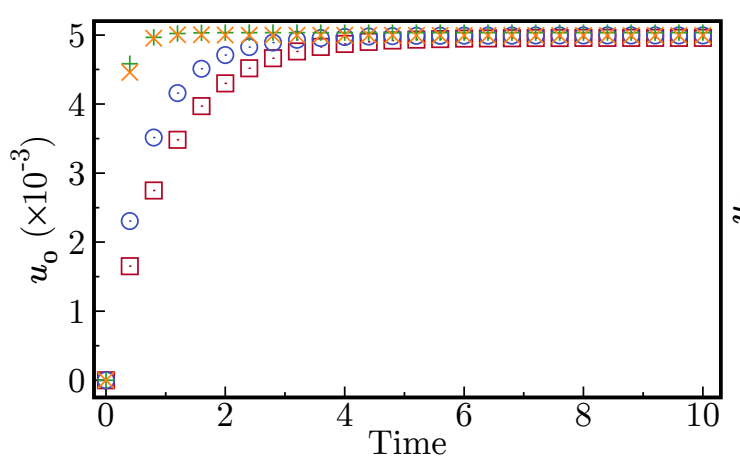

(a) Neutrals

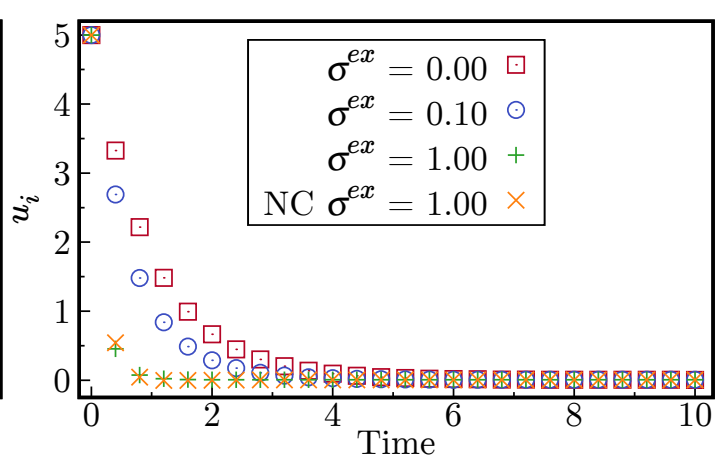

(b) Ions

Figure 4.24: $\quad$ Macroscopic velocity for both species for different constants values of $\sigma^{\text {ex }}$. Noncollisional case $(\mathrm{NC})$ is represented with orange crosses.

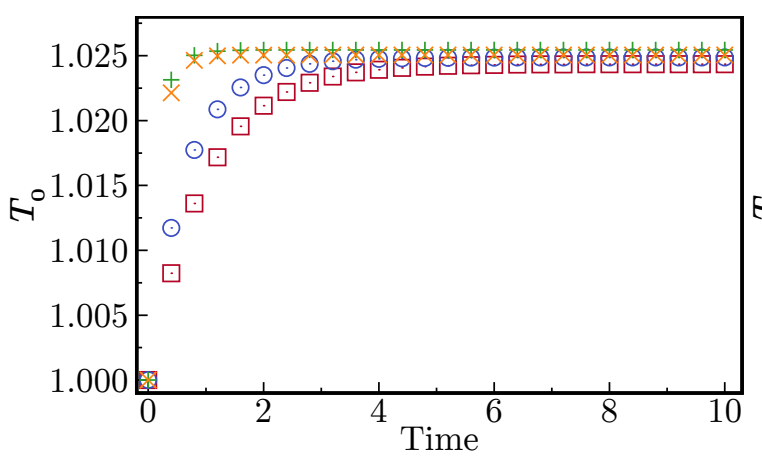

(a) Neutrals

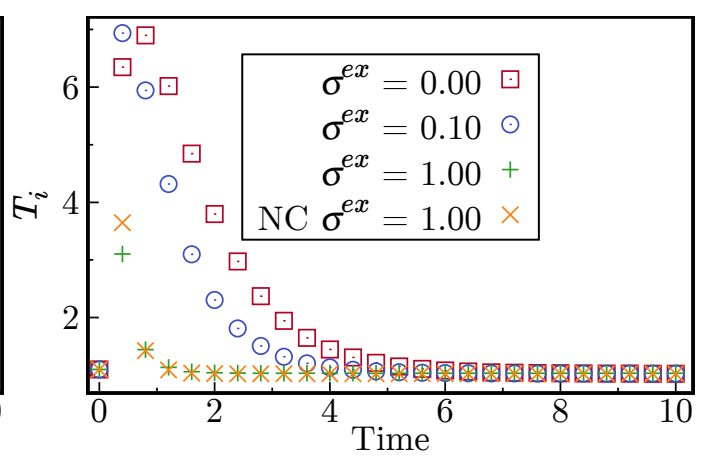

(b) Ions

Figure 4.25: Temperature for both species for different constants values of $\sigma^{\text {ex }}$.

initial one. Firstly, a zone of high ion temperature is reached, caused by the exchange of velocity with neutrals. Then, ion temperature reduces until a steady state for both species is reached. The maximum value of the peak temperature remains constant, independently of $\sigma^{e x}$, but the time required to reach it and then to reach a quasi-steady situation is highly modified by the charge-exchange operator. Again, a higher chargeexchange intensity result in faster relaxations. In figure 4.26, the distribution function for both species is pictured The neutrals do not experience an appreciable evolution, but $f$ moments exhibits a clear evolution for this species. Meanwhile, charge-exchange highly influence the ions distribution function shape. When this interaction becomes relevant to the system dynamics, higher $\sigma^{e x}$, a two peaked distribution function arise, while it is a Maxwellian for low values of $\sigma^{e x}$. Without elastic collisions, distribution function maxima are narrow, since elastic collisions relax faster the ion distribution function.

We have found that charge-exchange acts as an additional collision operator, exchanging energy and momentum between ions and neutrals. Nevertheless, the effect of this exchange in the distribution function leads to transitory situations far from ideal Maxwellian ones, as doubled peaked distribution functions arise if charge-exchange become a relevant phenomena. 


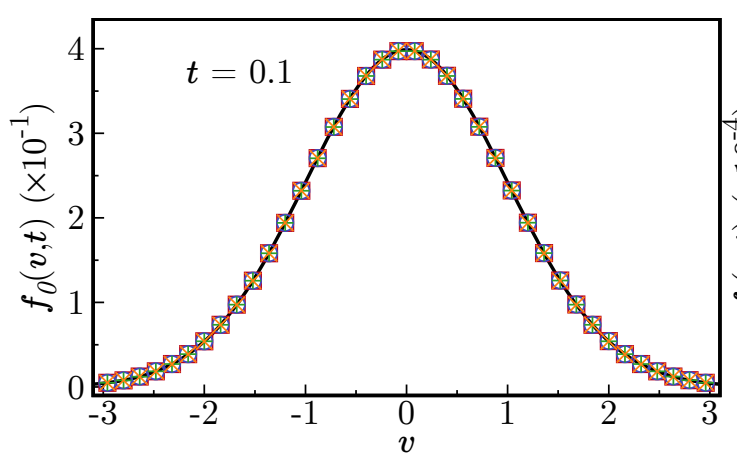

(a) Neutrals

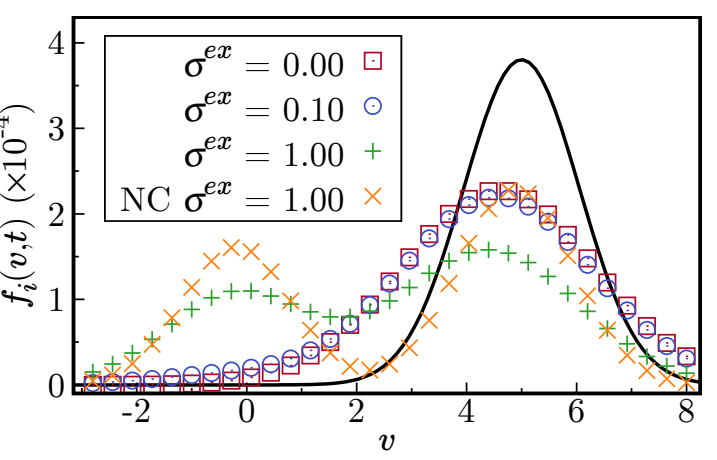

(b) Ions

Figure 4.26: Distribution function for both species at $t=0.1$ non-dimensional units. Initial condition is represented by a black solid line for both species.

\subsection{Conclusions}

Interactions among particles are of great importance in the study of plasmas and gases. They are the main cause of thermalisation and transport phenomena and their effects should not be neglected, except in very specific, well justified, situations or when the numerical or analytical tools available to solve the models required it. Even in cases when these interactions seem not to affect the first order macroscopic moments and fluxes, e.g., in the self-collisional case, they are necessary to provide a smooth and physical meaningful distribution function with the accurate entropy evolution and steady state.

In this chapter, some terms appearing in more complex models in this dissertation have been studied from analytical and numerical points of view. Special attention has been given to the study of conserved quantities, proving that the Propagator Integral Method provides solutions where the energy, momentum and norm loses are very small, in the order of the machine numerical error, and do not modify their conservative properties. This is valid for operators in the two and one velocity spaces. A study of the distributions functions have been performed, finding that the BGK approximation term may provide non-continuous solutions, resulting in a evolution where entropy is not maximised. On the other hand, the global effect of drift-diffusive operators provide an always smooth physical meaningful solutions. This can be easily analysed through the entropy evolution comparison, which also provides a physical foundation to select collision operators used in the development of kinetic models. The Propagator Integral Method can also deal with species of different temperature, mass, density and velocity ratios, employing the same time step to advance both distribution functions and keeping the total system energy constant thorough the simulations.

A peculiar term has been proposed to reproduce the Fokker-Planck-Landau behaviour when the problem is constrained to one-dimensional evolution. This term evolves the solution to a $\kappa$-like distribution function, as it can happen in some physical situations.

Another interaction phenomenon is the charge-exchange between ions and neutrals. 
This term acts as an extra collision operator from a macroscopic point of view, but $f$ with two, or more, maxima may arise if this term become relevant enough, e.g., if ions have a kinetic energy respect to neutrals. 


\section{CHAPTER 5}

\section{KINETIC DESCRIPTIONS OF WEAKLY IONIZED PLASMAS (WIP)}

Weakly Ionized Plasmas (WIP) are characterised by a low amount of charged particles respect to neutrals $[25,122-124]$. The ionization ratio, i.e., the relation between charged particle density and the total one, is usually in the range $10^{-8}-10^{-2}$ [56], which means that much more neutrals than charged particles exists. Usually, this kind of plasmas are generated in a laboratory but they can also be found in nature. These plasmas are normally cold and non-thermal, $T_{e} \neq T_{i}$, with electron temperature in the order of 1 to $10 \mathrm{eV}$ and its total density in the range of $10^{-12}-10^{-16} \mathrm{~cm}^{-13}$, which includes many kinds, as atmospheric plasmas [125] or space plasmas (solar wind, ionosphere or interstellar) [126]. This type of plasma is widely used for surface treatment $[5,6]$, electric propulsion [8] and medicine [57]. Its dynamics is of main interest in this dissertation to develop and solve kinetic models that improve the understanding of plasmas generated in the low temperature plasma laboratory of the UPM PlasmaLab ${ }^{1}$, along with the improvement of the ALPHIE electric thruster [100,127].

Modelisation of these plasmas is always a difficult task due to the large time scales, in the order of of $\mu$ s and ms, that arise due the inclusion of a heavy, dense, non-charged cold species, that represents more than the $99 \%$ of the particles in the plasma. New collision terms to account for the interaction of charged species with the large amount of neutrals must be included. These terms are usually neglected due to the short-distant interaction, but they become relevant in WIP due to the high density of neutrals acting as a viscous media $[24,28]$. Also, dynamics of the heavy species, as ions, is usually not accounted for, which may end up modifying the electron dynamics through the self-consistent electromagnetic field [101]. Many approaches have been proposed by

1 http://plasmalab.aero.upm.es/English.html 
different authors $[69,71,120,123]$ and by us [25] to describe dynamics of the charged species in weakly ionized plasmas, but usually require simplifications or the dumping of some dynamics and terms that may become relevant. In addition, non-linear effects as ionization or recombination, charge-exchange or interaction with a metallic wall are common when these cold plasmas are studied, increasing the complexity of the models and hindering the theoretical, numerical and experimental analyses.

It is not easy to define where the limits to consider a plasma as weakly ionized are set, as they depend on multiple factors, e.g., density of neutrals or energy of the charged species. As a general case, we can assume that a plasma is weakly ionized when it has enough charged particles to experience the perturbation produced by an electromagnetic field as a whole but also has enough neutrals so that charge-neutral interactions become relevant to the dynamics of the complete system. Of course, this property does not only depend on the plasma densities, but also on the species temperatures, velocities, and external forces that influence the system dynamics as a whole.

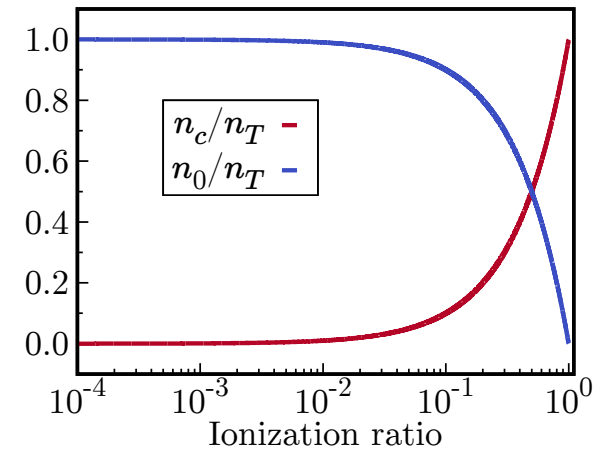

Figure 5.1: Representation of the charge and neutral densities respect to the total one as a function of the ionization ratio. An ionization ratio of 1 indicates a fully ionized plasma.
The ionization ratio $r_{i}$ defines the amount of charged particles in a plasma respect to the total one. This is an important parameter in WIP because the species densities influence the collisional exchanges and the self-consistent electromagnetic field. It is defined as

$$
r_{i}=\frac{\sum n_{\alpha}}{n_{T}}
$$

where subscript $\alpha$ represents charged species and $n_{T}$ is the total density of particles. Many authors, as for example U. S. Inan et al. [56], account only for the relation between electrons and neutrals to determine the ionization ratio, but we think that this description is more complete as there may arise situation when multiple charged species, as multi-charged ions, appear; as well as scenarios where the ion and electron densities do not coincide. If we assume a three species plasma with neutrals, electrons and single charged ions, the densities are defined by

$$
\begin{aligned}
\sum n_{\alpha} & =n_{i}+n_{e}=n_{c}=r_{i} n_{T} \\
n_{0} & =n_{T}-n_{c},
\end{aligned}
$$

where subscript 0 represents the neutral species. If a quasi-neutral plasma is assumed, ion and electron densities are equal to $\frac{n_{c}}{2}$. The ionization ratio can have values from 0 (no ionized, neutral gas) to 1 (fully ionized), and the charged number of particles evolves linearly with it. In figure 5.1, the density for charges (ions and electrons) and neutrals respect to the total one are pictured. This large amount of neutral particles has a high influence in the plasma dynamics as it interacts with the charged species $[12,25,95,117$, 128]. Nevertheless, due to the strong interaction between charged species, because of 
the large range of Coulombian interaction and the presence of the electromagnetic field a small amount of charges drastically changes the system dynamics. So, it is important to analyse and understand the plasma as a whole, where all the species move and interact at their different scales and with their peculiarities and exchange energy among them by means fo microscopic processes. Moreover, weakly ionized plasmas are usually cold and non-thermal, $T_{e} \sim 1-10 \mathrm{eV}$ while $T_{i}, T_{0} \ll T_{e}$, which means that characteristic time scales vary for each species in a wide range. These time scales are similar to those involved in experiments, which means that collisions are important and cannot be neglected in order to obtain a proper representation of the system dynamics [28]. The peculiarities of these complex systems lead to simplifications and fluid models that may not completely capture the microscopic dynamics of these systems failing to describe the physical behaviour of the problem

In this chapter a three species one-dimensional kinetic description for weakly ionized plasmas is presented, including a set of numerical resolutions and test performed to analyse the response of the model. This model, motivated by planar geometry usually employed to deal with simple descriptions of plasma phenomena in diagnosis, was firstly presented in [25], and it was further studied in $[28,112]$. Now it is expanded and studied in depth, including new numerical cases to better understand the WIP dynamics. In these results, we will explore the importance of the charge-neutral interaction in the dynamics of these plasmas, which become important due to the high density of the noncharged species, as well as the charge-charge influence, which may affect transitory and steady values. Also, the system response to an abrupt time changing electric field is analysed. Moreover, different scenarios of fixed ionization ratios are examined and changes in the plasma response is found for certain high values of $r_{i}$. To finalise with the study of this model, a discrepancy found in experimental data when a Langmuir probe is biased with different voltages signals is analysed.

To end with this chapter, a series of calculation of transport coefficients for WIP are presented. We propose to obtain transport coefficients by the self-consistent resolution of the distribution function influenced by collisional effects, external forces and variations in the spatial gradients. This approach allows the study of a large variety of cases and scenarios besides allowing an accurate computation of these coefficients, not based on linearisations or perturbation theory.

\subsection{One dimensional three species kinetic model}

To properly model weakly ionized plasmas, the effect of the neutrals on the charged species dynamics should be taken into account $[12,25,117,128]$. The high density of this species influences the dynamics of the plasma as a whole, modifying relaxation times and macroscopic states reached by the charged species due to electromagnetic forces. Also, due to their high mass and density and low temperature, neutral particles evolve in a slower time scale with respect to electrons, close to ion characteristic scale. 
Its evolution could modify the system dynamics when large times are analysed, e.g., when a measurement process takes place in the order of ms. This new scale adds an additional difficulty to numerically analyse these models, which may lead to inaccurate behaviours due to high ratios that appear in the convection and diffusion parameters.

Therefore, the dynamics of charged species may be ultimately governed by the presence of neutrals, and charge-neutral interactions may become the relevant phenomena to properly analyse the dynamics of these systems. On the other hand, fluid models may fail to describe the complex interactions that appear in a WIP, leading to incomplete or non-physical solutions. To provide a kinetic description of WIP that accounts for the disparate scales that naturally arise and the complex elastic exchange among the species, a three species one-dimensional in the velocity space model is presented. This model assumes an homogeneous space distributed plasma with an external applied electric field, which directs the dynamic of the complete system in one privileged direction. All elastic interactions among the species are accounted for. The capability of the PIM to deal with disparate scales allows conservative resolution of different plasma states and scenarios, advancing all species with the same time step.

The model consists on a system of three drift-diffusion equations for the evolution of the distribution functions of each species. This models includes the evolution of electrons, ions and neutrals, but more species could be added in a similar way without affecting the numerical simulations consistency. In this model, interaction terms as those presented in sections 4.1.4 and 4.1.5 are employed to represent charge-neutral and charge-charge collisions, respectively. The choice of Dougherty operators for the interaction with neutral particles is justified by the large amount of this species respect to ions and electrons. Due to the one-dimensional constrain imposed in this model, the charge-charge operator presented in section 4.1.5 provides a good approximation to the complete Fokker-Planck-Landau term, to represent the collision between charged species.

The equation that describes the evolution of a distribution function for a charged species $\alpha=e, i$ has the form

$$
\frac{\partial f_{\alpha}}{\partial t}+\frac{q_{\alpha} E(t)}{m_{\alpha}} \frac{\partial f_{\alpha}}{\partial v}=\sum_{\gamma}\left(\frac{\partial f_{\alpha}}{\partial t}\right)_{\alpha \gamma}+s_{\alpha},
$$

where $m_{\alpha}$ and $q_{\alpha}$ are the mass and charge of the species $\alpha$ respectively, $E(t)$ is the electric field, $\gamma=e, i, 0$ references any species in the mixture and $s_{\alpha}$ is a non-homogeneous term that represents source-sink effects. The same form also stands for neutrals by setting $q_{\alpha}=0$. The term $\left(\partial f_{\alpha} / \partial t\right)_{\alpha \gamma}$ symbolizes the rate of change of the distribution function due to collisional effects between species of kinds $\alpha$ and $\gamma$. Each of these exchanges are modelled by a drift-diffusion operator, in the Fokker-Planck from (4.1), as

$$
\left(\frac{\partial f_{\alpha}}{\partial t}\right)_{\alpha \gamma}=-\frac{\partial}{\partial v}\left\{D_{v}^{\alpha \gamma}-\frac{\partial}{\partial v} D_{v v}^{\alpha \gamma}\right\} f_{\alpha}
$$

The parameters $D_{v}^{\alpha \gamma}=D_{v}^{\alpha \gamma}\left(f_{\alpha}, f_{\gamma}, v, t\right)$ and $D_{v v}^{\alpha \gamma}=D_{v v}^{\alpha \gamma}\left(f_{\alpha}, f_{\gamma}, v, t\right)$ are referred to 
the non-linear convection and diffusion coefficients, respectively. The values for $D_{v}^{\alpha \gamma}$ and $D_{v v}^{\alpha \gamma}$ are those presented in 4.1.4 for the charge-neutral collision and in 4.1.5 for the charge-charge interaction.

The evolution of neutrals is described by

$$
\frac{\partial f_{0}}{\partial t}=\sum_{\gamma}\left(\frac{\partial f_{0}}{\partial t}\right)_{O \gamma}+s_{0} .
$$

No external forces for this species are assumed, meaning distribution function evolution, and its macroscopic values, is governed by exchange among the species and non-homogeneous terms.

As it will be explained in section 5.1.1, charge-neutral interactions are of huge importance in weakly ionized plasmas since they drive the dynamics of the plasma as a whole. Nevertheless, all collisions terms are included in this model because some situations may appear when charge-charge collisions become important. Moreover, even when its influence is small, different states are reached if these terms are not accounted for. Furthermore, the model has the capability to incorporate a time variable external electric field. This field adds energy to the charged species, which can be transferred to neutrals through collisional effects. This means that all the species in the mixture are affected by the electric field the perturbation, directly or indirectly $[24,25]$.

The complete coupled system of equations describing the evolution of plasma species is

$$
\begin{aligned}
\frac{\partial f_{e}}{\partial t}-\frac{e E(t)}{m_{e}} \frac{\partial f_{e}}{\partial v} & =\sum_{\gamma}\left(\frac{\partial f_{e}}{\partial t}\right)_{e \gamma}+s_{e} \\
\frac{\partial f_{i}}{\partial t}+\frac{e E(t)}{m_{i}} \frac{\partial f_{i}}{\partial v} & =\sum_{\gamma}\left(\frac{\partial f_{i}}{\partial t}\right)_{i \gamma}+s_{i} \\
\frac{\partial f_{0}}{\partial t} & =\sum_{\gamma}\left(\frac{\partial f_{0}}{\partial t}\right)_{0 \gamma}+s_{0}
\end{aligned}
$$

where $e=\left|q_{e}\right|$ is the elementary charge. As a whole, nine collision terms are included in the model. The charged species have self-collisions $(\alpha-\alpha)$, interactions with the other charged species $(\alpha-\beta)$ and interactions with neutrals $(\alpha-0)$. Neutrals have three Dougherty type interaction modes including self-collisions and collisions with both charged species.

The collisions parameters are defined as

$$
\mu^{\alpha \beta}=\frac{4 \pi \lambda^{\alpha \beta} q_{\alpha}^{2} q_{\beta}^{2}}{m_{\alpha}^{2} V_{t h}^{\alpha 2}} ; \mu^{\gamma 0}=\sigma^{\gamma 0} V_{t h}^{\gamma},
$$

where $V_{t h}^{\gamma}=\sqrt{\frac{k T^{\gamma}}{m-\gamma}}$ is the thermal velocity in the one-dimensional space, $\lambda^{\alpha \beta} \sim 10-20$ is the Coulomb's logarithm, where approximated values can be taken from the formulary 


\begin{tabular}{lcll}
\hline & Mass $(\mathrm{g})$ & Temperature $(\mathrm{eV})$ & Density $\left(\mathrm{cm}^{-3}\right)$ \\
\hline$e$ & $9.10938356 \times 10^{-28}$ & 1 & $r_{i} \frac{n_{T}}{2}$ \\
$i$ & $72819.6 \times m_{e}$ & $0.05 \times T_{e}$ & $n_{e}$ \\
0 & $m_{e}+m_{i}$ & $0.03 \times T_{e}$ & $n_{T}\left(1-r_{i}\right)$ \\
\hline
\end{tabular}

Table 5.1: Mass, temperature and density employed to represent an argon WIP in the three species one-dimensional model.

of J. D. Huba [129]

$$
\begin{aligned}
& \lambda^{e e}=23.5-\ln \left(n_{e}^{\frac{1}{2}} T_{e}^{-\frac{5}{4}}\right)-\left[10^{-5}+\frac{\left(\ln T_{e}-2\right)^{2}}{16}\right]^{\frac{1}{2}} \\
& \lambda^{e i}=23-\ln \left(n_{e}^{\frac{1}{2}} T_{e}^{-\frac{3}{2}}\right)
\end{aligned}
$$

and $\sigma^{\gamma 0}$ is the total cross-section of the interaction $\gamma$ - 0 , extracted from [130, Chapter II, Section 14]. The cross-sections for the charge-neutral exchanges are constant and equal to $\sigma^{i 0}=10^{-14} \mathrm{~cm}^{2}$ and $\sigma^{e 0}=10^{-16} \mathrm{~cm}^{2}$. The neutral-neutral cross-section is equivalent to the ion-neutral one given the similarity between both particles. It can be seen how collision parameters depend on the species temperature through the thermal velocity, the Landau length and the total cross-section. Nevertheless, other definitions for these parameters could be employed.

The electric field is necessary to apply this model, as shown in 4.1.5, to ensure the validity of the charge-charge interaction term and also, because in an absence of boundary conditions or non-homogeneous terms that feed the plasma to keep the quasi-neutrality, all the initial energy will be distributed among the species, reaching situations where electrons become cold enough to allow a high rate of recombination, driving the plasma towards a neutral gas after some relaxation time.

For the applications presented in this work, parameters of the argon gas, table 5.1, are used with initial Maxwellian distribution functions. For the cases presented in this part, unless stated otherwise, the total density equals $n_{T}=10^{13} \mathrm{~cm}^{-3}$ and $r_{i}=10^{-6}$.

To properly solve this model, it is advisable to work, from a numerical point of view, in non-dimensional units, as previously done. Due to the disparate time scales that appear in the model, it is important to choose the correct reference values to avoid having some terms so small that do not affect the numerical problem or, on the contrary, so big that break the simulation. The reference parameters, labelled by superscript 0 , chosen in this work for the dimensionless problem are

$$
\begin{gathered}
n^{0}=n_{0} ; m^{0}=m_{e} ; q^{0}=q_{e} ; T^{0}=T_{e} ; \\
\sigma^{0}=\sigma^{e 0}=10^{-16} \mathrm{~cm}^{-2} ; v^{0}=V_{t h}^{e} ; \\
\mu^{0}=\sigma^{0} v^{0} ; \nu^{0}=n^{0} \mu^{0} ; t^{0}=\nu^{0-1} ; E^{0}=1 \mathrm{~V} / \mathrm{m} .
\end{gathered}
$$


The choice of the electron-neutral collision as a characteristic parameter provides a good compromise between the high density of neutral particles and high energy of electrons. The same procedure as with the Vlasov equation in section 3.3 is performed now, including the rescaling of the collision frequencies as $\tilde{\mu}^{\alpha \beta}=\mu^{\alpha \beta} / \mu^{0}$ and $\tilde{\mu}^{\gamma 0}=\mu^{\gamma 0} / \nu^{0}$.

From a numerical point of view 5001 points for each species with different grid lengths ( $L_{e}=100, L_{i}=0.05$ and $L_{0}=0.03$ ) are employed. These different grids are chosen to ensure that all distribution functions are properly represented. Time step equals $\tau=10^{-2}$, for a reference time of $t^{0}=2.384 \cdot 10^{-2} \mathrm{~ms}$, small enough to ensure that the conservation properties of all collision terms are fulfilled.

\subsubsection{Influence of collisions in the dynamics of WIP}

When collisions are added into plasma descriptions, usually only the interaction between charges is accounted for. The interaction between charged and neutral particles is neglected or assumed as an interaction with a static background due to the low density of neutrals and small effective cross section, i.e., low intensity of particle-particle interaction. This is valid, for example, for high density and temperature plasmas, where characteristic collisional times are several orders of magnitude below the problem scales and plasma can be considered as instantaneously thermalised. Moreover, this approximation is also accurate when characteristic time and length scales are order of magnitude below those related with the collision processes. However, in weakly ionized plasmas, neutrals play a fundamental role and can significantly modify the evolution of the charged species. In this type of plasmas, charge densities are small and electrons are relatively cold (in the order of $1-10 \mathrm{eV})$, which means that characteristic time

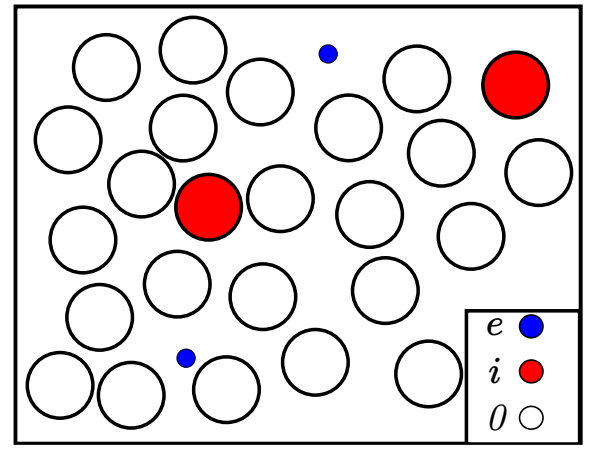

Figure 5.2: Representation of amounts of neutrals (white circles), ions (red circles) and electrons (blue circles). scales are usually in the range of micro and milliseconds, similar to the times studied in WIP.

As explained above, charged species represent only a small fraction of particles in WIP. In figure 5.2, a scheme of a typical distribution of particles in a three species (neutrals, single charged ions and electrons) plasma is pictured. It can be seen how the massive presence of neutrals will make charged particles interact more with this kind of particles than with other charged species (or themselves). Even when this interaction has a small cross-section, because it is a short-range collision instead of a large-range one as the Coulombian interaction, the cumulative effect of collisions acts as a viscous media where charged particles move and exchange momentum and energy to the neutral species. This means that neutrals become important due to their high 


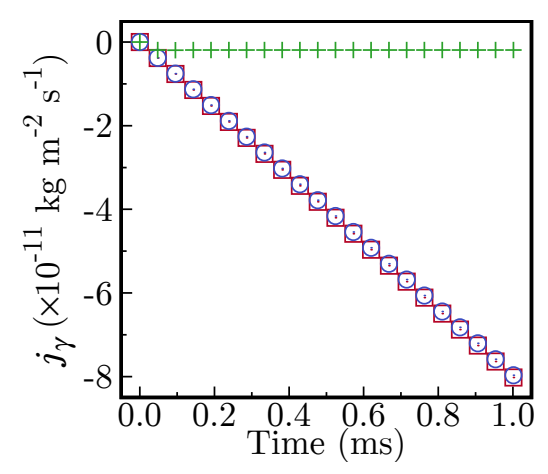

(a) Electrons

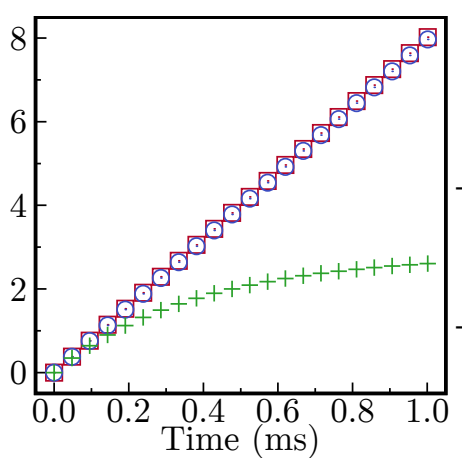

(b) Ions

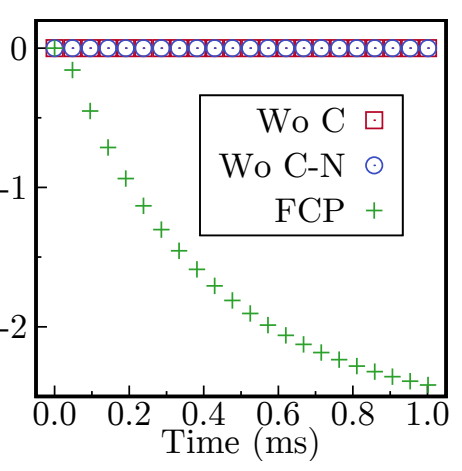

(c) Neutrals

Figure 5.3: Evolution of mass fluxes for (a) electrons, (b) ions and (c) neutrals. Three types of plasmas are studied: without collisions (Wo C), collisional plasma without chargeneutral interaction (Wo $C$-N) and a fully collisional plasma (FCP).

density with respect to charged species, and its evolution can be relevant to describe the plasma as a whole, specially in large time-scales, as we presented in references $[24,25]$ and was also stated by many authors in recent years $[12,95,117,128]$.

Collision terms are highly related to the particle density and the energy of the species, whether through the collision frequency or by the distribution functions directly, as seen in chapter 4 . Weakly ionized plasma have low ionization ratios, which means that neutrals have a large particle density respect to the charged species. Hereby, the interaction of charged species with neutrals become relevant, due to the high density of the last one which act as a viscous background. Specially electron-neutral collision may become of major importance due to the high energy of electrons, meaning that a larger amount of energy can be exchanged between both species.

A first topic that must be addressed to understand the dynamics of weakly ionized plasmas is the influence of the charge-neutral exchange. The effects of these terms are numerically studied and compared with classical non-collisional descriptions. A three species argon plasma is considered under the effect of a constant electric field of value $0.1 \mathrm{~V} / \mathrm{m}$ in three cases: a non-collisional plasma, a plasma without the chargeneutral interaction and the complete model presented in the section above. The model employed for the non-collisional plasma is the one presented in (5.7) but with collision and non-homogeneous terms set to zero. Even when the macroscopic moments for this simple space homogeneous Vlasov problem can be obtained analytically for a known electric field [25], here it will be solved with the PIM for one-dimensional non-diffusive equations to test the numerical accuracy of the scheme. Also, solving the three cases numerically ensures similar sources of errors, which helps the data comparison. The resolution of both collisional models is performed with the PIM for open 1D driftdiffusion equations.

Figure 5.3 shows the evolution of the mass flux $j_{\gamma}=\left[m_{\gamma}\right]$ for all the species. Huge differences among the three scenarios are found. While behaviours for collisionless and 


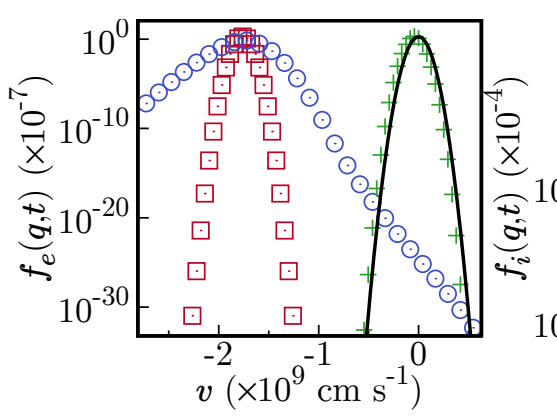

(a) Electrons

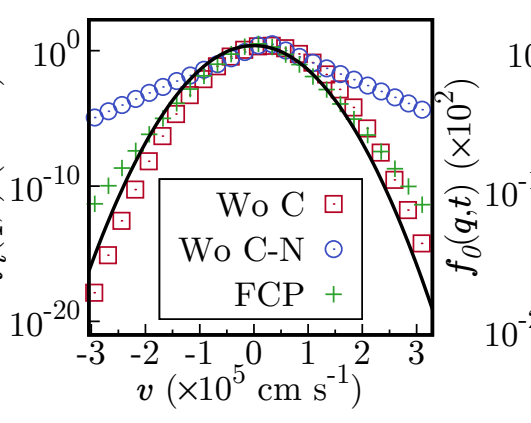

(b) Ions

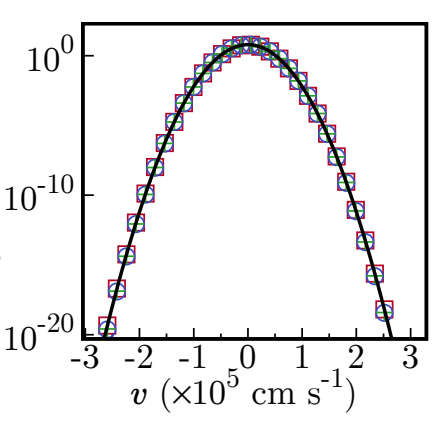

(c) Neutrals

Figure 5.4: Distribution function at $t=1 \mathrm{~ms}$ is presented for (a) electrons, (b) ions and (c) neutrals. Black solid line represents the initial Maxwellian condition.

collisional plasma without charge-neutral interactions are very similar, the addition of neutral effects to the mixture completely changes the dynamics of the system. Fluxes for charged species are reduced because part of the energy transmitted by the electric field is transferred to the neutrals through elastic collisions. Because of this, neutrals evolve and gain velocity, acting as a sink of energy, a behaviour that does not occur in the first two models. If these collisions are neglected, charged species accelerate due to the electric field without any restriction since electron-ion interaction is not strong enough to constrain the system evolution. The high rate of exchange between electrons and neutrals also makes both fluid velocities of the same sign, i.e., neutrals and electrons move in the same direction. In a first study of the this problem, it may be expected that neutrals follow the ions evolution, due to the similar masses of both species. However, the low thermal velocity of this heavy species produces very low exchanges with neutrals.

Collisional terms also modify the shape of the distribution function, as seen in figure 5.4. In the non-collisional case, distribution function does not change and just moves as a whole due to the effect of the electric field. Only when the effect of charge-charge interactions is introduced, an important change in the distribution function is found becoming non-Maxwellian due to the effect of the one-dimensional interaction. For the fully collisional plasma, we can observe how high energy tails still hold, specially for ions, due to the action of the one-dimensional charge-charge operator, generating a power-law tail dependence, as a $\kappa$ distribution function. Electrons have an almost Maxwellian distribution due to the high influence of the electron-neutral collisions. This term, in addition with fast thermal velocity of electrons, produce a fast relaxation but also means that an important amount of energy gained by the electrons due to the action of the electric field is transferred to neutrals. Hence, in presence of an important amount of neutrals, the acceleration of charged species requires a high electric field, due to the viscous-like action of the neutral species.

In resume, we have established that charge-neutral interactions are important and drive the evolution of a weakly ionized plasma for the conditions presented in table 5.1. A final check still remains to be performed: the analysis of the charge-charge interaction 


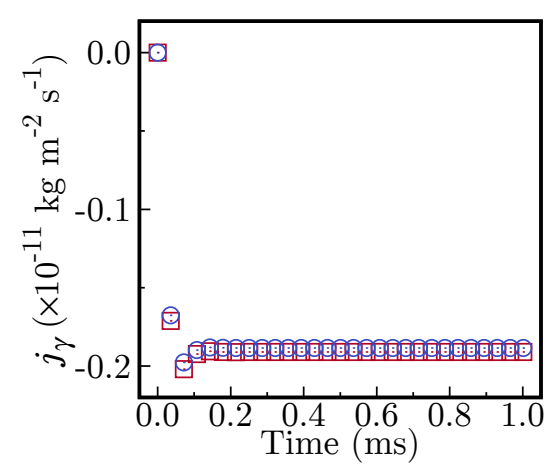

(a) Electrons

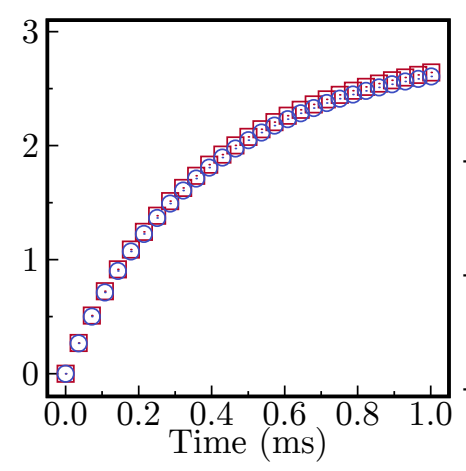

(b) Ions

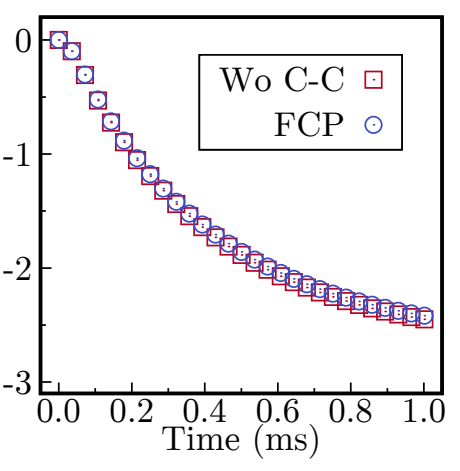

(c) Neutrals

Figure 5.5: Evolution of mass fluxes for (a) electrons, (b) ions and (c) neutrals. The fully collisional plasma (FCP) and a simulation where all charge-charge interactions have been disabled (Wo $C$-C) are presented.

in the plasma dynamics. These collisions have a huge cross-section due to the large range Coulomb interaction, but their effect is reduced in WIP due to the low density of electrons and ions. Figure 5.5 presents the mass fluxes for electrons, ions and neutrals for the model studied in this section and the same model with charge-charge interactions disabled. Almost same evolutions are reached for both examples, and only a small difference in the fluxes are obtained. Nevertheless, these differences could be more relevant in other situations, such as different ionization ratio or electric field. It is important, if the computational means allows it, to include all collisional terms, but as a first approach only charge-neutral interaction should be considered to properly describe the evolution of weakly ionized plasmas. This provides a proper macroscopic evolution of the system as charge-neutral interaction lead the weakly ionized plasma dynamics.

\subsubsection{Abrupt electric field}

Here, the three species kinetic model is tested with a time variable abrupt electric field, defined as

$$
E(t)=\left\{\begin{array}{rl}
E_{0} & \text { if } t<2.5 \mathrm{~ms} \\
-E_{0} & \text { if } t \geq 2.5 \mathrm{~ms}
\end{array},\right.
$$

where $E_{0}=0.1,0.2$ and $0.3 \mathrm{~V} / \mathrm{m}$. This particular shape is motivated because plasma usually evolves under abrupt changes, as for example when a sweep signal is introduced by means of a probe to measure its properties. Firstly, this electric field accelerates the charged species in one direction and then in the opposite one for $t \geq 2.5 \mathrm{~ms}$. The study of the evolution of a weakly ionized plasma under this abrupt change is interesting to understand some behaviours found in the laboratory, where fast sweep voltage signals are introduced for plasma diagnosis. Due to the abrupt electric field change, plasma requires a certain time to adapt to the new situation. Here, relaxation time refers to the time required by one species to reach an almost constant velocity after the electric 


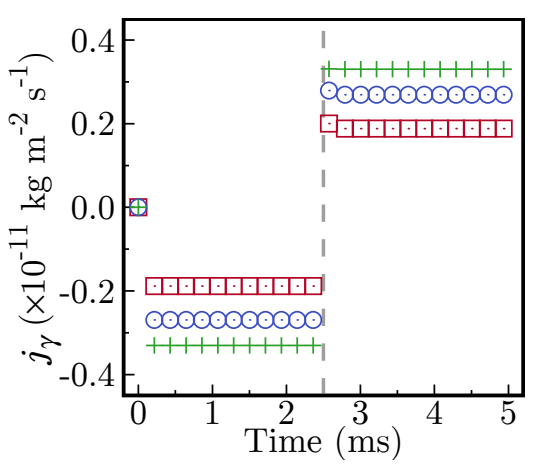

(a) Electrons

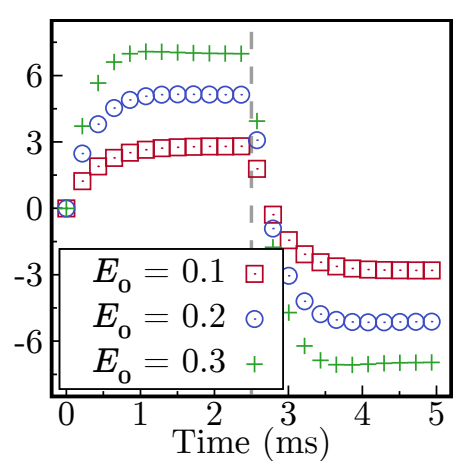

(b) Ions

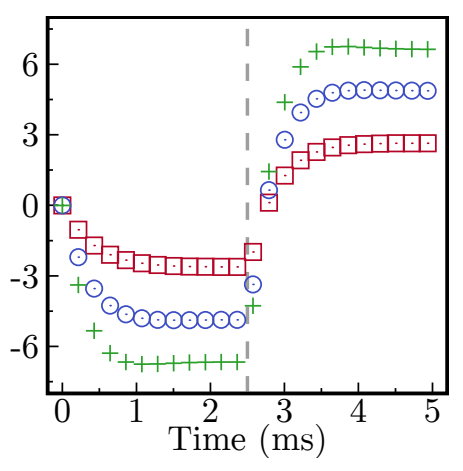

(c) Neutrals

Figure 5.6: $\quad$ Mass fluxes for (a) electrons, (b) ions and (c) neutrals. Values of $E_{0}$ are expressed in $\mathrm{V} / \mathrm{m}$.

field changes its direction.

In figure 5.6, the mass fluxes for the three species are presented. It is important to remark that the abrupt change in the electric field does not produces oscillations or instabilities in the time evolving numerical solution. In these results, different dynamics between the fast (electrons) and the slow (ions and neutrals) species arise naturally. First of all, electrons only require fractions of millisecond to recover a constant velocity. A magnified view of figure 5.6a is presented in figure 5.7 to better represent this fast change. Even when only fractions of $\mathrm{ms}$ are required by the electrons to adapt to the new electric field, the change is not instantaneous.

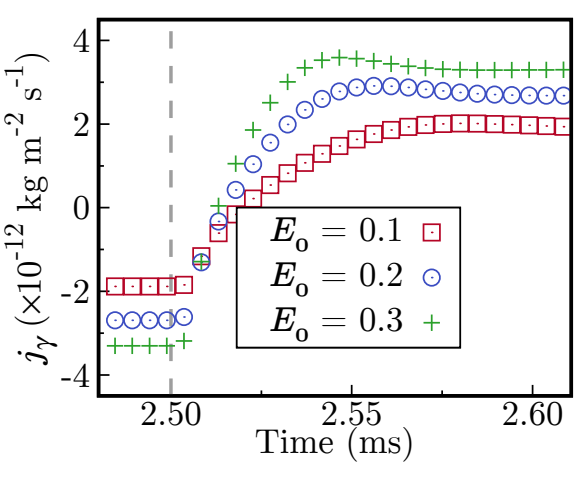

Figure 5.7: $\quad$ Zoomed view of figure 5.6 a when electric field changes. Values of $E_{0}$ are expressed in $\mathrm{V} / \mathrm{m}$.

An overshoot appears for high values of $E_{0}$, meaning that electrons reach a maximum of velocity which is higher than the one in the quasi-steady state. This overshoot in the mass flux of electrons grows with the electric field, but electrons recover quickly a constant velocity, meaning the electric field modifies the rate of exchange among particles thanks to the change in temperature [28]. On the other hand, ions and neutrals require several milliseconds to reach a steady state.

These distinguishable behaviours are directly related to the exchange rate of energy between charged species and neutrals. As it was indicated in table 5.1, electrons high temperature, and therefore higher thermal velocity than ions $\left(V_{t h}^{e} \gg V_{t h}^{i}\right)$. The chargeneutral collision frequency $\nu^{\gamma 0}$ is directly proportional to the thermal velocity, so that, the rate of electron-neutral exchange is higher than the ion-neutral one. This results in a faster relaxation time, even when $\sigma^{e 0}<\sigma^{i 0}$ and $m_{i} \sim m_{0}$. However, this fragile balance could change if different initial conditions are employed, e.g., with different temperatures or species masses. 


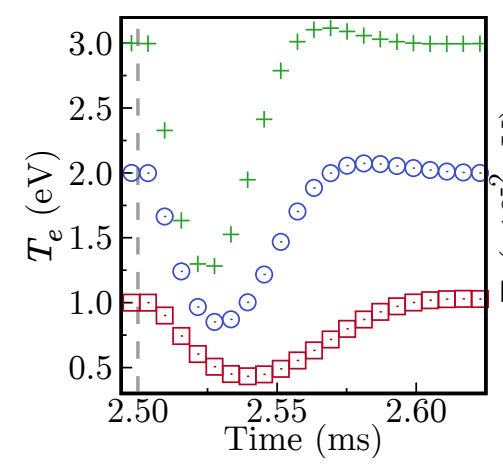

(a) Electrons

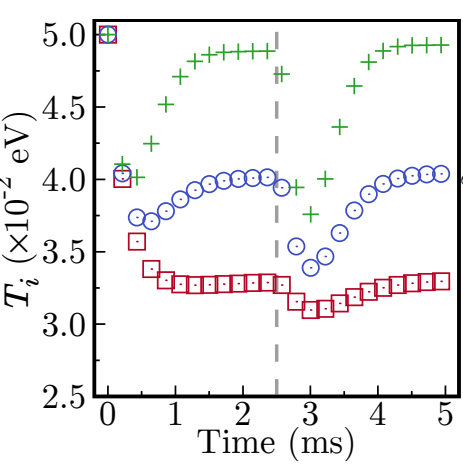

(b) Ions

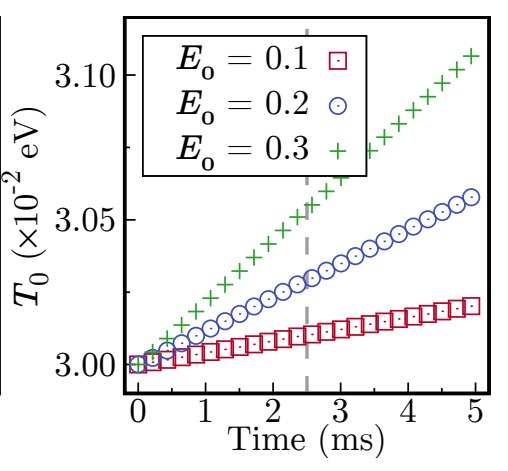

(c) Neutrals

Figure 5.8: Evolution of species temperatures for different values of $E_{0}($ in $\mathrm{V} / \mathrm{m})$. Higher temperatures are reached for more intense electric field, influencing collision frequencies and relaxation times.

The computation of the complete distribution function allows an accurate calculation of temperature for each species, including the possibility of high energy tails, as it may happen for charged species. To understand the dynamics of the charge species (specially for the electrons) as the electric field increases, it is important to remark that chargeneutral interaction frequency $\nu^{\gamma 0}$ is directly related with the species thermal velocity, i.e., more temperature means more energy is exchanged with neutrals. We present now the time evolution of the temperature in figure 5.8. Both charged species reach a higher temperature for a more intense electric field. A low temperature zone after the abrupt change at $t=2.5 \mathrm{~ms}$ appears, reducing the charge-neutral exchanges. However, the time for which temperature drops is completely different for each case, as it decrease with the increment of external field. For low values of $E_{0}$, charged species take a long time to recover a constant temperature. On the other hand, relaxation times of the electron mass flux are reduced at a higher $E_{0}$. The higher temperature reached by the charged species also allows this quickly recovery when the electric field changes. A temperature overshoot appears for electrons when electric field increases, as it happens in the flux. This is caused by the low temperature reached when the electric field changes, which influence the electron-neutral exchange.

\subsubsection{Temperature dependent collision frequencies}

To test the influence of the non-constant collision parameters $\mu^{\alpha \beta}$ and $\nu^{\gamma 0}$ employed in previous simulations, the same test case presented in section 5.1.2 is computed here keeping the initial values of these parameters through all the evolution. This is an usual approach to reduce the complexity of the problems and to avoid possible numerical instabilities for extreme temperatures. The mass flux of electrons is studied in figure 5.9 for different values of $E_{0}$ for temperature dependent ant constant collisional parameters. A difference between both dynamics is appreciated. When collision parameters depend on the species energy, an overshoot in the electrons mass flux appear due to the decrement in temperature presented in figure 5.8a. This decrement translates into 


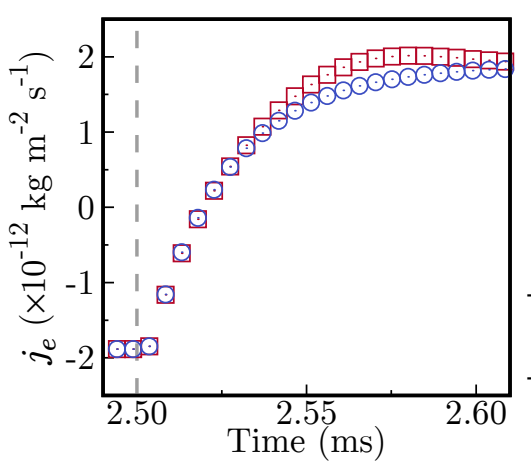

(a) $E_{0}=0.1 \mathrm{~V} / \mathrm{m}$

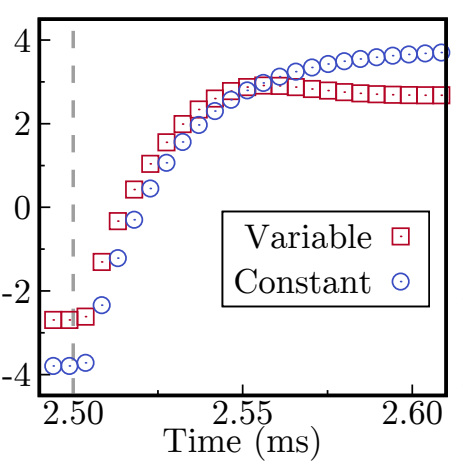

(b) $E_{0}=0.2 \mathrm{~V} / \mathrm{m}$

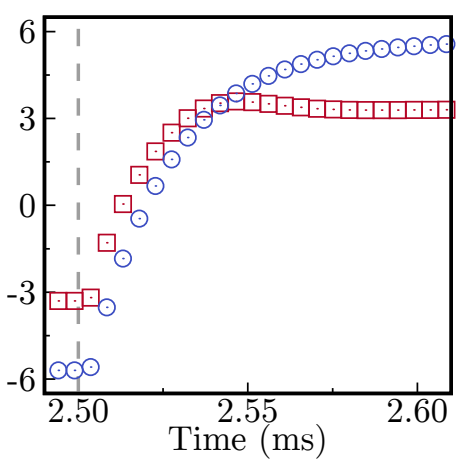

(c) $E_{0}=0.3 \mathrm{~V} / \mathrm{m}$

Figure 5.9: Comparison of the electron mass flux with variable and constant collision parameters. No overshoot happens with constant collision parameters.

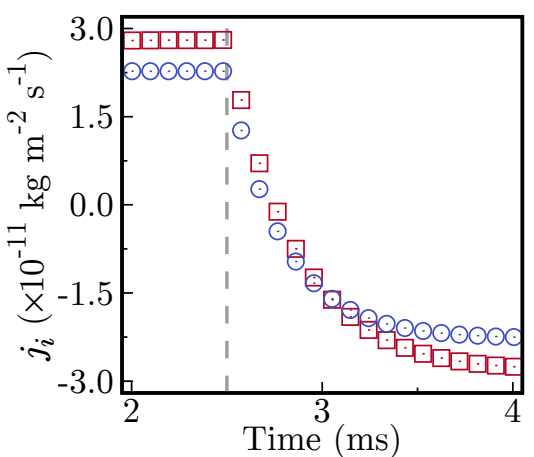

(a) $E_{0}=0.1 \mathrm{v} / \mathrm{m}$

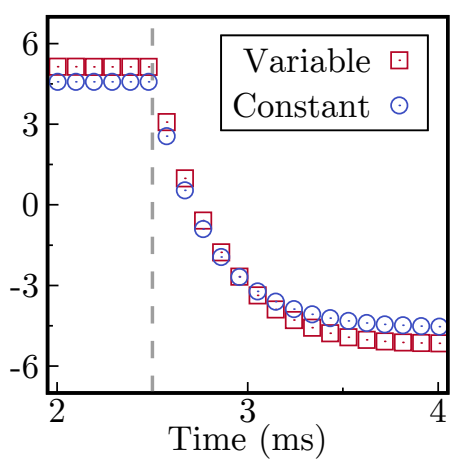

(b) $E_{0}=0.2 \mathrm{~V} / \mathrm{m}$

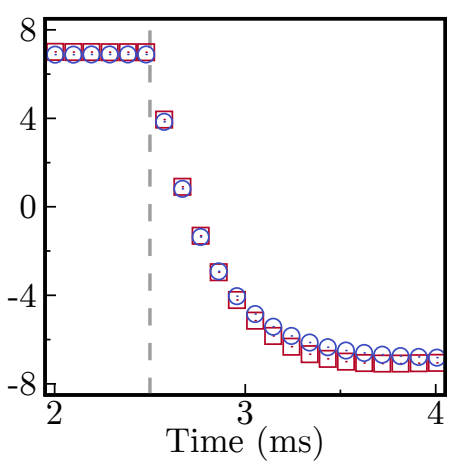

(c) $E_{0}=0.3 \mathrm{~V} / \mathrm{m}$

Figure 5.10: Comparison of the ions mass flux with variable and constant collision parameters. Small differences appear between both cases.

less exchanges with neutrals, which means the electric field has a higher influence on the charged species dynamics until temperature increases again, allowing it to reach higher velocities. On the contrary, with constant collision frequencies, no change or overshoot in the dynamics of the species appears as $E_{0}$ increases because, even for low species temperatures, electrons interact with neutrals at a constant rate, which results in a monotonic evolution until the maximum velocity is reached. Furthermore, as $E_{0}$ increases, differences in the steady states growth, because the constant collisional parameters simulations do not account for the temperature variations produced by higher electric fields.

In the same manner, the effect of the constant collision frequencies in the ion flux can be studied. Its study is of mayor importance, e.g., to the analysis of an electric thruster [100] and its evolution should be included in the models if the computational resources allow it. Furthermore, its dynamics affects the evolution of a plasma as a whole, specially the self-consistent electric field when a charge separation appears [101]. Figure 5.10 shows the behaviour of ions with constant and temperature dependent collision parameters. Even when the change in both dynamics is small, some differences in the steady situation reached also appear, which could affect the dynamics of this 
heavy species. Due to the small variation of ion temperature with $E_{0}$, the differences in their mass fluxes are small and do not vary as the electric field increases.

It is clear that temperature influences the transitory and quasi-steady dynamics in WIP, specially in those situations where the electric field changes abruptly or temperature varies. These results indicate that keeping collision parameters as their initial (or any other constant) value could lead to inaccurate results in these or other situations. Nevertheless, the approximation of constant collision parameters could still provide close descriptions of the plasma dynamics if these parameters are chosen carefully.

\subsubsection{Ionization ratio}

As said, the ionization ratio in a WIP can vary in a range that goes up to $10^{-2}$, where plasma can be considered as fully ionized. This ratio determinates the relation between the density of charged particles $n_{c}$ and the total one $n_{T}$. For the three species plasma dealt in this section, the ionization ratio presented in equation (5.1) becomes

$$
r_{i}=\frac{n_{c}}{n_{T}}=\frac{n_{e}+n_{i}}{n_{0}+n_{e}+n_{i}} .
$$

To study the effect of this parameter in the plasma dynamics and also, to test the limits of the model presented above, some scenarios at different ionization ratio are studied. The electric field presented in (5.12) is employed here. Some initial results about this parametric analysis were presented in [112], where a linear relation between $r_{i}$ and the mass fluxes was found up to a specific degree of ionization. Here, the study is extended to analyse also how the intensity of the electric field and the total plasma density modify the dynamics and the behaviours at high degrees of ionization.

First, let us start reviewing the results obtained in the paper cited above. In that work, a range of $r_{i}$ from $10^{-7}$ up to $10^{-4}$ was studied for $n_{T}=10^{13} \mathrm{~cm}^{-3}$ and $E_{0}=0.1 \mathrm{~V} / \mathrm{m}$. A linear response of the mass fluxes was found for $r_{i} \leq 10^{-5}$. In the analyses presented here, additional values of $r_{i}$ are utilised to better understand the influence of this parameter in the plasma dynamics and try to determine a better limit for the linear zone. Figure 5.11 presents the mass fluxes for the three species at $r_{i}=10^{-6}, 10^{-5}$, $3 \cdot 10^{-5}$ and $10^{-4}$. A first relevant result that comes up is that absolute value of the flow increases for all the species as the ionization ratios increase. This fact is justified for electrons and ions due to the raising of their particle densities and mean velocity, thanks to the action of $E(t)$, whereas the neutral mass flow is enhanced by two effects. Firstly, as higher the electron flow is, more intense the electron-neutral interactions become, and, secondly, as lower $n_{0}$ is, the neutrals acquire the transferred energy by the charges but sharing it in a fewer number of particles. Consequently, we can assert that a balance exists between the decreasing neutral density, resulting in less electron-neutral interactions, and the increment in energy of the charged species, which means that higher momentum and energy can be exchanged between electrons and neutrals. The interplay of both effects generates the flow of neutrals high enough even for relatively 


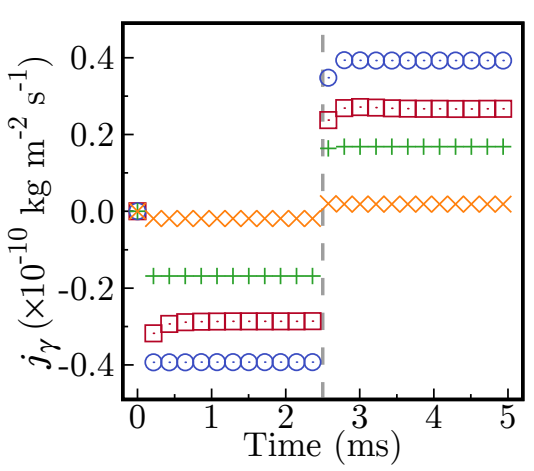

(a) Electrons

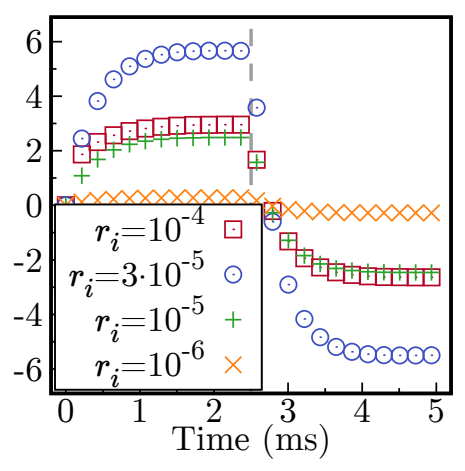

(b) Ions

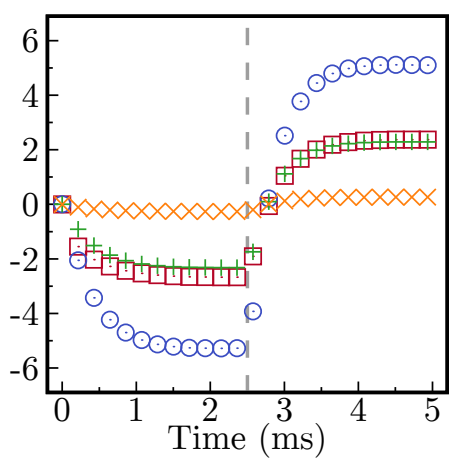

(c) Neutrals

Figure 5.11: Mass fluxes for the three species for $r_{i}=10^{-6}, 10^{-5}, 3 \cdot 10^{-5}$ and $10^{-4}$. A maximum value is found for $r_{i}=3 \cdot 10^{-5}$.

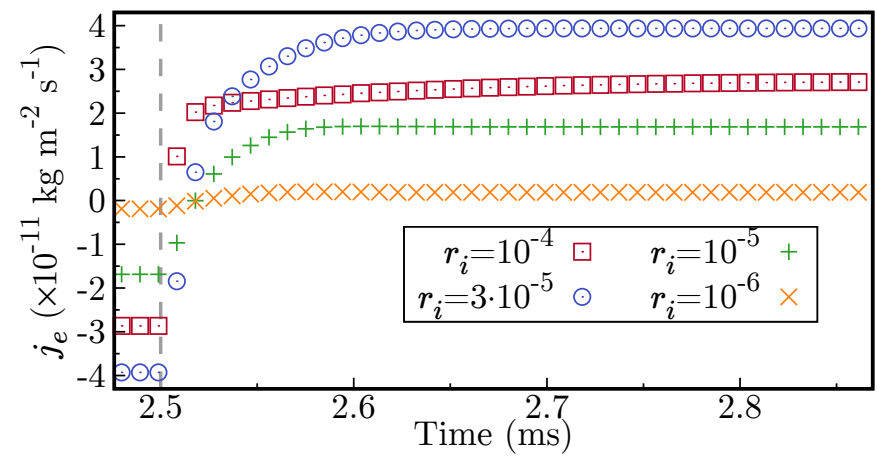

Figure 5.12: Zoomed view of figure 5.11a when electric field changes. A change in the electron dynamics for $r_{i}=10^{-4}$ is clearly appreciated.

large $r_{i}$. However, this balance and almost uniform behaviour dramatically changes at high values of $r_{i}$ because the neutral flows at $r_{i}=10^{-5}$ and $r_{i}=10^{-4}$ are very similar, specially after the abrupt change of $E(t)$. Therefore, it is expected that for much higher degrees of ionization the neutrals flow vanishes in the limit of a fully ionized plasma. The ions undergo a similar tendency for the highest values of $r_{i}$ presented here. With respect to the electrons, due to their low inertia, they reach an almost quasi-steady in just fractions of millisecond, then, the electrons flow, shown in figure 5.11a, provides no substantial information within the evolution close to $2.5 \mathrm{~ms}$.

To improve the analysis of electrons dynamics, figure 5.12 presents a magnified view of the mass electron flux immediately before the field turns off its direction. Very similar dynamics emerge as electrons accommodate very fast and smoothly to a constant mean velocity when $E(t)$ changes. However, for $r_{i}=10^{-4}$ a quite different behaviour is found since the observed smooth adaptation of the other cases seems to disappear for this particular degree of ionization, for which the electrons reach the steady velocity faster than in the previous situations. For lower $r_{i}$ ratios, electrons take up to $0.5 \mathrm{~ms}$ to reach their final quasi-steady velocity. Hence, the reduction of $n_{0}$ decreases the number of electron-neutral interactions allowing the electrons to adapt themselves faster to the new electric field, evolving as if no interaction with the neutrals occurs. Nevertheless, 


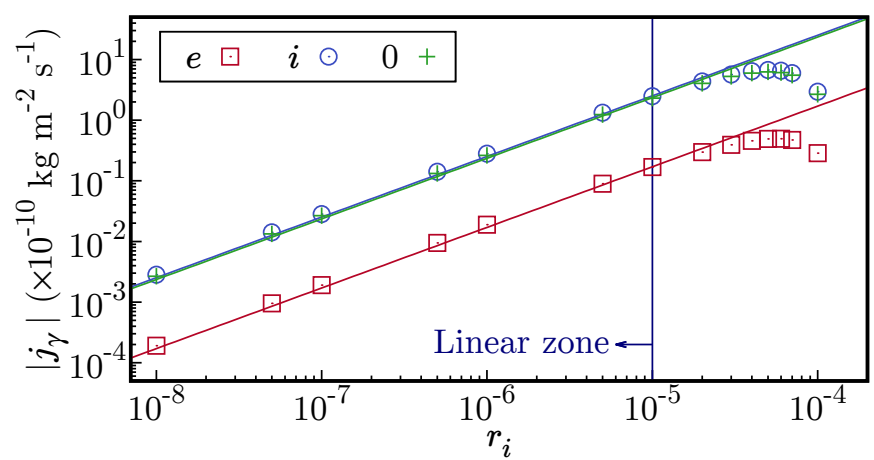

Figure 5.13: Flux before the change in electric field as a function of the ionization ratio. Linear regressions are represented with solid lines.

once this relative high velocity is established, the interactions with neutrals become relevant again, a fact that favours the electrons to evolve slowly to a quasi-steady state presented in figure 5.11a. This effect does not appears for heavy charges, since ions reacts slowly to the electric field change showing a smooth dynamics for any analysed $r_{i}$.

On the other hand, to understand the quasi-steady state, figure 5.13 depicts the mass fluxes just before the change of the electric field as a function of the ionization ratio. This graph is improved by adding some extra values of $r_{i}$ for very low ionization ratios and in the range between $r_{i}=10^{-5}$ and $10^{-4}$, where plasma presents a critical change in its behaviour. A linear zone appears for $r_{i} \lesssim 10^{-5}$ where the steady mass fluxes of all species behave linearly with the ionization ratio. This linear behaviour is clearly distorted beyond the linear zone, since the fluxes decay with a non apparently predictable law for $r_{i} \geq 5 \cdot 10^{-5}$. These reduction of the charges flows indicates that an increase of electron and ion densities does not imply an enhancement of their mass fluxes and the plasma does not behaves as expected with a linear growth of the current density, for instance. Accordingly, it can be assumed that for ionization ratios over $r_{i}=10^{-4}$ the fluxes decrease and the charge-neutral collisions become less relevant to the whole plasma dynamics. The maximum $r_{i}$ that defines the end of this zone may change as a function of other plasma properties and processes, although we consider the previous results hold partially ionized plasmas close to steady states with specified $r_{i}$.

A final interesting remark comes out from the analysis of the non-linearity of the collision parameters, $\mu^{\alpha \beta}$ and $\mu^{\gamma 0}$ with respect to the species kinetic temperatures, a fact that can be significant for the dynamics, as shown in [28]. In figure 5.14, the electron and ion transient temperatures are pictured for the more relevant $r_{i}$ values. The abrupt change in $E(t)$ reduces the charged species temperatures evolving in a very similar form as happens for the ionization ratios corresponding to what we have named linear zone, though some differences arise even inside this zone. In particular, an overshoot, i.e. a higher temperature with respect the one found before $E(t)$ variation, appears in electron temperature for and $10^{-6}$ that vanishes for $10^{-5}$. This phenomenon appears as a consequence of the drastic change in the electron-neutral collision parameter $\mu^{e 0}$ 


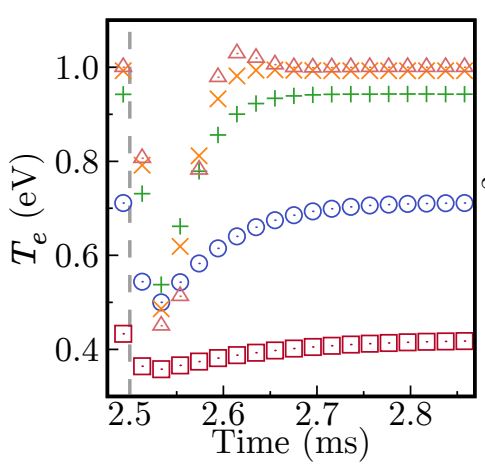

(a) Electrons

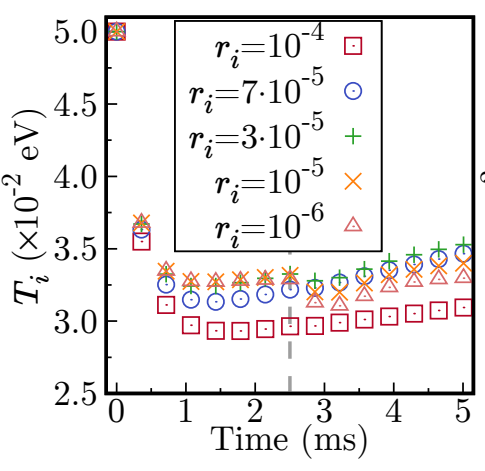

(b) Ions

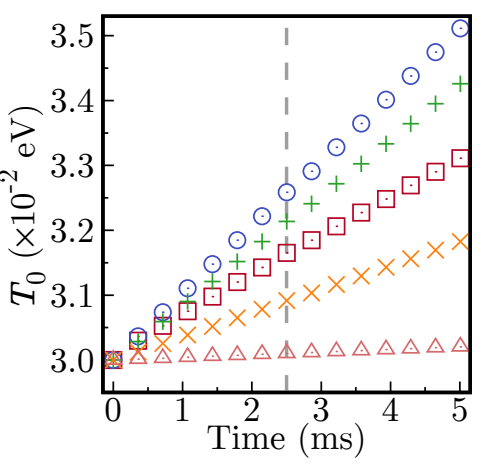

(c) Neutrals

Figure 5.14: Temperature of the three species for $r_{i}=10^{-6}, 10^{-5}, 3 \cdot 10^{-5}, 7 \cdot 10^{-5}$ and $10^{-4}$. A drop in the electron temperature appears at high ionization degrees.

which is not significant for low neutral density since the energy exchanges become less intense, eliminating the overshoot. The ion temperature evolves indistinguishably for $r_{i}=10^{-6}$ and $10^{-5}$, while it slightly increases for higher $r_{i}$, specially after the $E(t)$ change. Surprisingly, quite different behaviours are found for $r_{i}=10^{-4}$ in both charged species. The electron temperature deviates form its initial order of magnitude of $1 \mathrm{eV}$ decreasing up to $\sim 0.4 \mathrm{eV}$. In this state, recombination with ions is more likely, which might produce the reduction of the ionization ratio. Neutral temperature reaches a maximum for $r_{i}=7 \cdot 10^{-5}$, but an always increasing evolution is found. However, higher electric fields could enlarge the linear zone extent, preserving the linear response of fluxes for higher degrees of ionization.

In conclusion, starting from a WIP and increasing the ionization ratio results in a non-linear response for certain values of $r_{i}$. This response, in addition, results in a important drop of electron temperature, which may result in recombination with neutrals. However, dynamics related with fully ionized plasmas, where charge-charge interactions become an important phenomena, arise for certain ionization ratios and dynamics presented here are valid only for the value of $E_{0}$ and $n_{T}$ used in the simulations. As said in section 5.1.2, dynamics of the three species are influenced by the value of electric field, reaching higher temperatures and different transient dynamics. It is expectable that a higher $E_{0}$ also influences the plasma dynamics at different scenarios. A variety of ionization ratios are analysed with $E_{0}=0.3 \mathrm{~V} / \mathrm{m}$, keeping $n_{T}=10^{13} \mathrm{~cm}^{-3}$. As a result of this increment, we conjecture that the increment of energy provided to the system can modify the linear zone range. Figure 5.15 represents the steady mass fluxes at different ionization ratios. It can be observed how the limit of the linear zone is shifted to the right, meaning that a higher degree of ionization can be reached for the system an the expected behaviour of a WIP remains. If a higher energy is given, temperature and velocity of all the species increase, as it was presented in section 5.1.2. This high energy allows to sustain the linear behaviour of the plasma up to $r_{i} \simeq 3 \cdot 10^{-5}$. However, a similar behaviour that the one found for $E_{0}=0.1 \mathrm{~V} / \mathrm{m}$ is also matched for higher ionization ratios. 


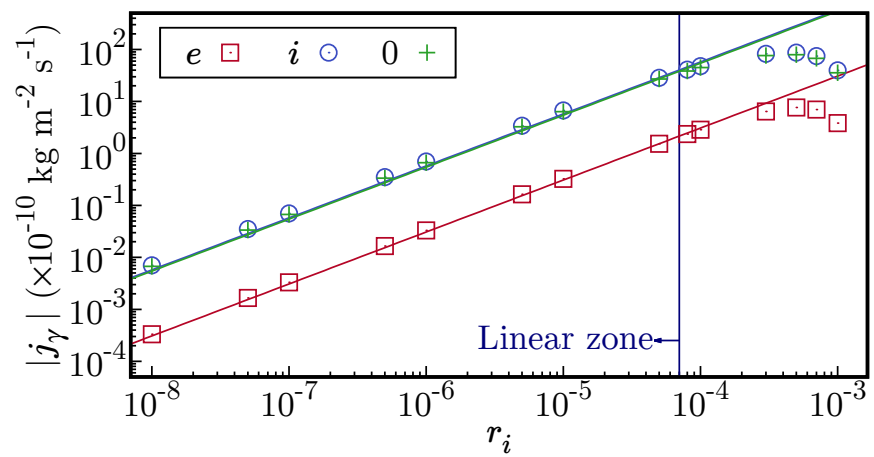

Figure 5.15: Steady fluxes versus the ionization ratio for $E_{0}=0.3 \mathrm{~V} / \mathrm{m}$. The range of the linear zone has increases due to the increment in the electric field.

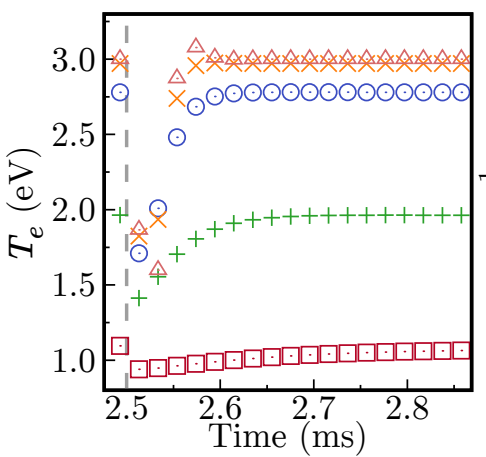

(a) Electrons

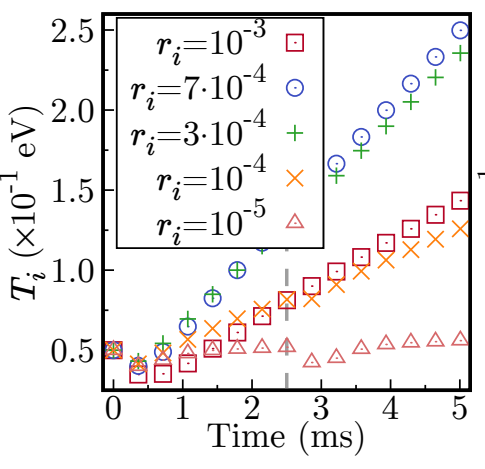

(b) Ions

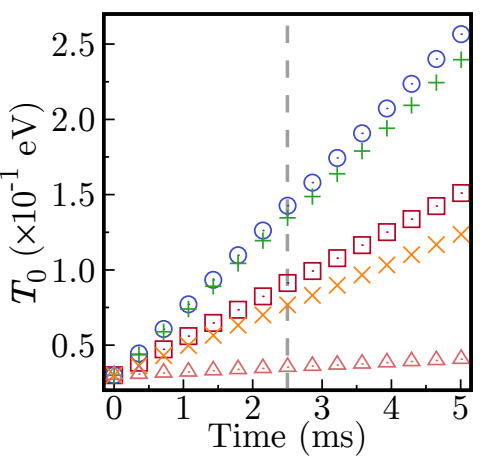

(c) Neutrals

Figure 5.16: Temperature of the three species for $r_{i}=10^{-5}, 10^{-4}, 3 \cdot 10^{-4}, 7 \cdot 10^{-4}$ and $10^{-3}$. Ion temperature increases for high degrees of ionization, acting as an energy sink.

Figure 5.16 represents the time evolution of the species temperatures for $r_{i}=10^{-5}$, $10^{-4}, 3 \cdot 10^{-4}, 7 \cdot 10^{-4}$ and $10^{-3}$. A first interesting result is that electron temperature drops to $\sim 1 \mathrm{eV}$, which is far from the recombination zone. This means that, for this electric field intensity, the weakly ionized plasma will not become a neutral gas, even if the ionization ratio increases close to $1 \%$. On the other hand, a relevant behaviour is found for neutrals and ions. In previous simulations, the neutral temperature always increases, meaning that this high density species acts as a sink, gaining part of the energy transmitted to the charges by the electric field thanks to the elastic collisions. For a higher $E_{0}$ situation firstly we see how temperatures of both heavy species drastically augment, reaching almost ten times the values found in previous cases. Here, ion temperatures increase abruptly respect to low ionization ratios, and keep a positive slope for almost all time, and no steady state is reached. This means that, in this situation, ions act as another sink of energy, as it may happen in fully ionized plasmas. These non-steady situations may also indicate that the system require more time to adapt to the new electric field, as they become more energized. Also, the large energisation of neutrals could result in a part of it being ionized, modifying the ionization ratio.

Another parameter that is expected to modify the plasma dynamics is the total density of particles, $n_{T}$. As it happens when $r_{i}$ increases, the particle density of charged 


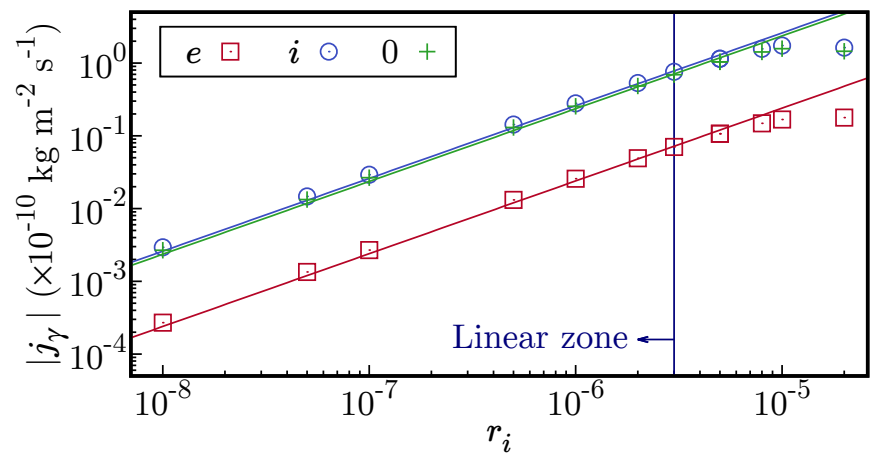

Figure 5.17: Steady mass fluxes for $n_{T}=2 \cdot 10^{13} \mathrm{~cm}^{-13}$. The maximum value of the linear zone shifts to the left respect to the case of $n_{T}=10^{13} \mathrm{~cm}^{-13}$.

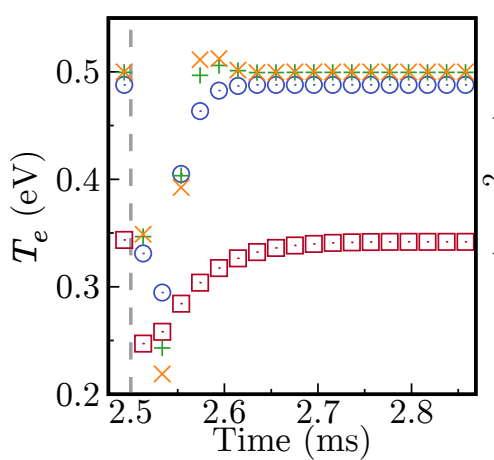

(a) Electrons

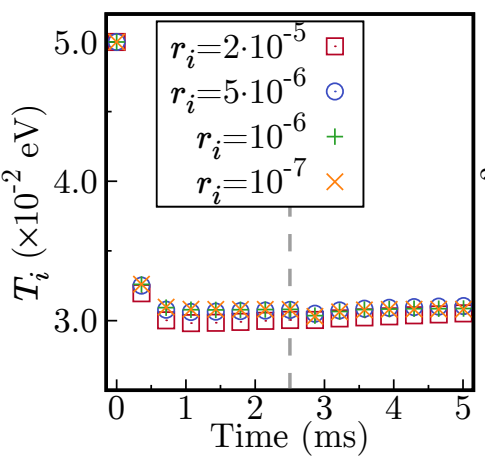

(b) Ions

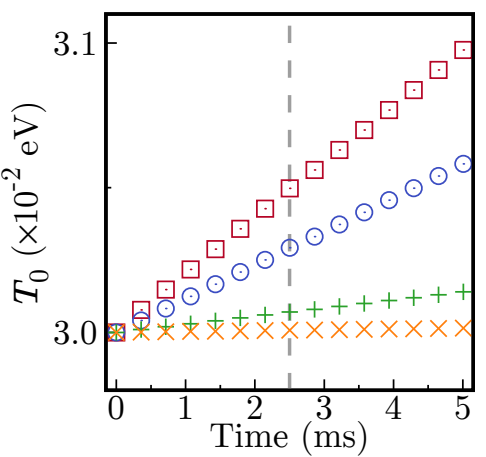

(c) Neutrals

Figure 5.18: Temperatures for the three species with a total density of $2 \cdot 10^{13} \mathrm{~cm}^{-13}$. At high ionization ratios, electron temperature drops and ion and neutral temperatures greatly increase.

species also varies due to an increment of the total number of particles. Thence, it is expected that that lower values of the previous linear limits are reached for higher $n_{T}$. Figure 5.17 pictures the steady fluxes for a value of total density of $n_{T}=2 \cdot 10^{13} \mathrm{~cm}^{-13}$ and $E_{0}=0.1 \mathrm{~V} / \mathrm{m}$. It can be seen how the steady fluxes keep a linear variation with $r_{i}$ up to $r_{i} \sim 3 \cdot 10^{-6}$. This limit is below those found in previous cases. Therefore, we can assert that to maintain a linear behaviour in more dense plasmas, a higher electric field is required. For this scenario of $n_{T}$, temperatures are lower than in previous cases, as shown in figure 5.18, reaching for all cases situations where a full recombination with ions is possible. In consequence, we are in position to establish that to obtain a higher total density plasma, more energy must be added to the system, for example, imposing a higher electric field or using a source of hot electrons.

As it happened for $n_{T}=10^{13} \mathrm{~cm}^{-3}$, a higher electric field also extends the range of the linear zone. Let us analyse now the case with a total density of $2 \cdot 10^{13} \mathrm{~cm}^{-3}$ and $E_{0}=0.3 \mathrm{~V} / \mathrm{m}$. In figure 5.19 it can be seen how the maximum value of validity for linear zone moves forward to the right, as an effect of the high electric field. However, the maximum degree of ionization for the linear case is still lower that the one presented in figure 5.15, meaning that even a higher input of energy is required to reach a linear zone limit close to $r_{i}=10^{-4}$. 


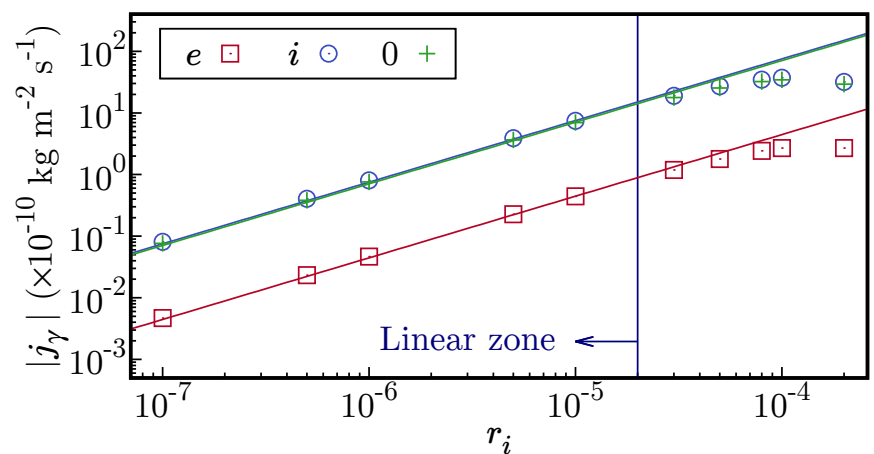

Figure 5.19: Steady mass fluxes for $n_{T}=2 \cdot 10^{13} \mathrm{~cm}^{-13}$ and $E_{0}=0.3 \mathrm{~V} / \mathrm{m}$. In this situation, the maximum value of the linear zone also shifts to the right.

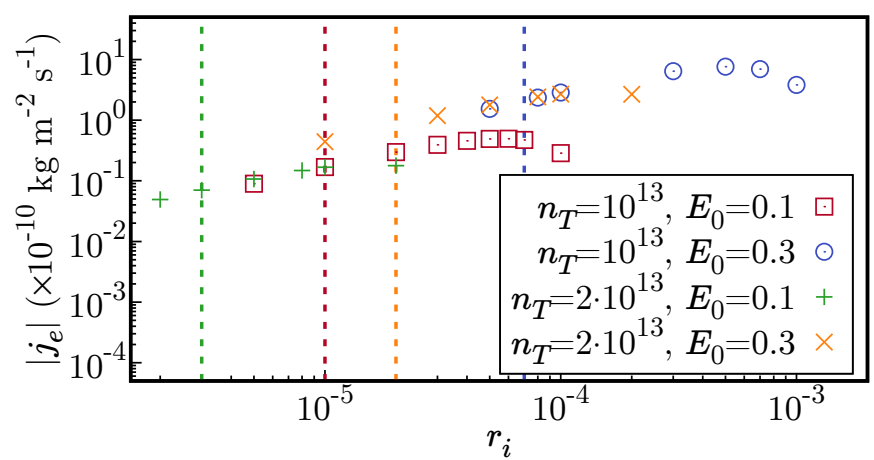

Figure 5.20: Fluxes at the end of the linear zone for the different cases studied in this section. Total densities are expressed in $\mathrm{cm}^{-3}$ and electric field in $\mathrm{V} / \mathrm{m}$. Dashed lines represent the end of the linear zone for each case.

Figure 5.20 shows the fluxes in the surroundings of the linear zone limit for the different values of total density and electric field studied here. Dashed lines represent the degree of ionization that marks the end of the linear zone for each scenario. As the electric field increases, the limit of the linear zone also does, so, whereas an increment a higher total density shift the limit to lower $r_{i}$. So, to get a higher total density plasma, that behaves as a weakly ionized one, it is required a more intense electric field to increase the linear zone.

As results presented here prove, plasma behaves linearly up to a ionization degree, in which a change in the plasma dynamics appears. Since this change appears as the amount of charges increases respect to neutrals, it is reasonable to assume that the variation is caused by the charge-charge collisions, as they are directly related with the electron and ion densities. Figure 5.21 pictures the steady mass fluxes presented in figure 5.13 including relevant scenarios of different $r_{i}$ without accounting for the chargecharge collisions. Without charge-charge interactions the linear evolution with $r_{i}$ holds for situations where mass fluxes decay if these processes were accounted for. Therefore, as the amount of electrons and ions increases, plasma behaves closer to a fully ionize one where exchange between charges become the dominant process. For low values of $r_{i}$ charge-charge interactions have low impact in the plasma dynamics, as presented in figure 5.5. This modification in the plasma dynamics can only be found by the self- 


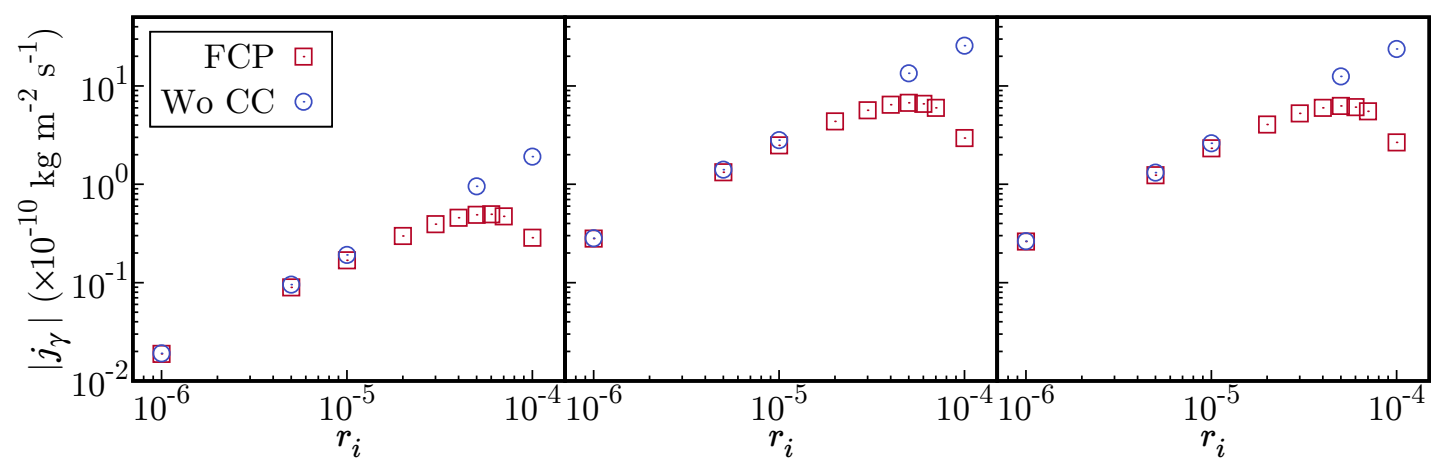

(a) Electrons

(b) Ions

(c) Neutrals

Figure 5.21: Steady fluxes with (FCP) and without (Wo CC) charge-charge collisions for $n_{T}=10^{13} \mathrm{~cm}^{-3}$ and $E_{0}=0.1 \mathrm{~V} / \mathrm{m}$. Without the charge-charge interactions, plasma keeps a linear dynamics with $r_{i}$.

consistent resolution of the three species model, accounting for all collisional terms. Effects that are small for certain situations may become relevant in other scenarios and their dumping must always be justified. As self-collisions (electron-electron and ion-ion) do not modify macroscopic values, the variation in the plasma response can only be caused by the electron-ion collision, which becomes the dominant exchange that defines the plasma evolution.

We can conclude that the three-species model studied here provides steady situations that evolve linearly with the ionization ratio up to a maximum which depends on the electric field applied and on the total density of particles. Situations may appear where electron temperature drops to a value were recombination with ions becomes highly probably, meaning that the plasma may evolve to a scenario where a steady ionization ratio, different from the initial one, is found; or even evolve to become a neutral gas. This behaviour change is caused by electron-ion exchange becoming a relevant phenomena, meaning that plasma behaves closer to a fully-ionized one and that an important part of the electron energy is transferred to ions. While quasisteady situations inside the linear zone are easy to predict for any value of $r_{i}$, transitory evolutions may be more involved since overshoots or sharp dynamics may appear when the electric field changes.

\subsection{Analysis of certain discrepancies on the characterisa- tion of WIP by means of Langmuir probes}

As a relevant physical case, the three-species one-dimensional model presented in this chapter is used to analyse some not well understood results found in the laboratory when a weakly ionized plasma is characterized by means of a Langmuir probe. A Langmuir probe consists of an electrode immersed in a plasma, which collects charged particles depending on its bias voltage. These probes can be biased to different po- 
tentials, by means of a sweep signal, and the corresponding values of the collected current can be measured. As it is well-known, the representation of the bias voltage and collected current is called an I-V curve. The I-V curves of collecting probes provide information about parameters such as the local plasma potential, the electron or ion temperatures and the charged species density [131-133]. These probes are widely used for the diagnostics of fully [134-136] and weakly ionized plasmas [137-139]. Theoretically, the operation of Langmuir probes does not depend on the shape of the signal used to bias the probe. Therefore, similar plasma parameters should be obtained when different signals are employed in a stable plasma. However, the experimental results presented in this section show that the plasma parameters provided by signals with different symmetry or frequency do not lead to similar profiles. Consequently, this creates an open topic for discussions on which signal symmetry and frequency should be applied to the collecting probe to obtain the correct plasma parameters. This feature has been addressed in our paper [28], where a preliminary analysis of this problem was presented. As it can be seen, this experimental dilemma requires a better comprehension of the biasing of Langmuir probes from the theoretical point of view. A deeper analysis is performed here to improve the experiments and numerical simulations in future works. To analyse the possible microscopic interaction in the probe surroundings that give rise an appreciable effect in the measurement process, a kinetic model that accounts for all possible interactions among the particles must be employed. The propose of this analysis is twofold: firstly, to obtain a similar qualitatively behaviour as the experimental curves, and secondly, to identify the cause for the results discrepancies without the employment of new experimental techniques.

To allow a self-consistent lecture, the experimental setup employed to obtain the I-V curves is concisely explained. Results obtained by means of this setup are then presented. Then, the results obtained from numerical simulations are given and compared to the experimental data. To compare both data, the experimental I-V and the numerical $\mathrm{J}_{e}$-E curves, where $J_{e}=\left[q_{e}\right]$, are employed in a qualitatively examination. The causes of the discrepancies found in the experimental results are widely discussed. The ion current is studied separately, giving some arguments about the analysis of these heavy species with Langmuir probes and how it can influence its measurement. Due to the high mass of this species, orders of magnitude above the electron mass, ions require a larger time to adapt to the perturbation of the electric field, which results in the requirement to use lower sweep frequencies.

\subsubsection{A short review of the experimental setup and results}

In the experimental setup employed to obtain the results presented below in this section, the plasma was generated by means of a ring cusp ion thruster, operated with an argon gas flow, as that described in $[99,100]$. The density and temperature ranges of the electrons in the cold plasmas involved were $n_{e}=\left(10^{7}-10^{8}\right) \mathrm{cm}^{-3}$ and $T_{e}=$ $(0.75-1.25) \mathrm{eV}$, respectively. The Langmuir probe used, shown in figure 5.22a, was 


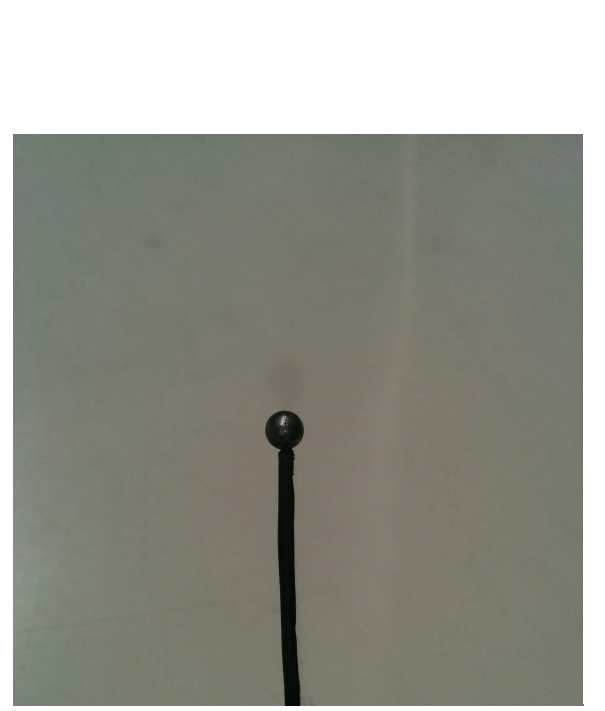

(a) Langmuir probe

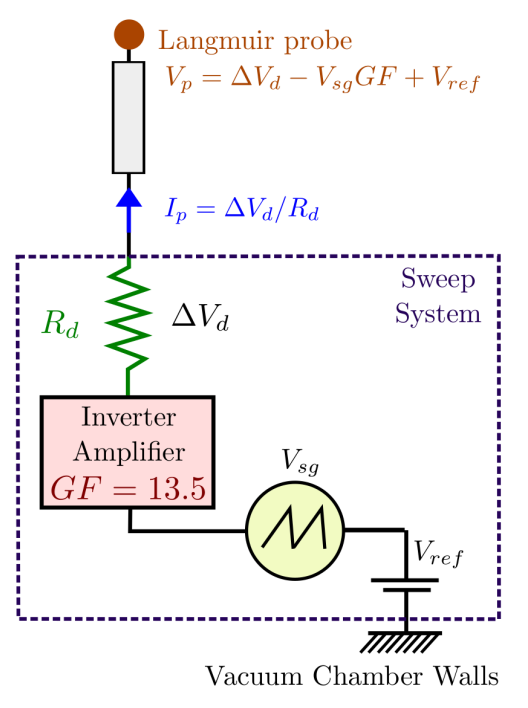

(b) Electric circuit

Figure 5.22: (a) Langmuir probe utilized for these measurements. (b) Electric circuit used to bias the probe with a sweep signal. With full permission from [140].

made of a stainless steel ball with $4.39 \mathrm{~mm}$ of diameter, and was placed at $37 \mathrm{~cm}$ from the thruster exhaust. The I-V curves were obtained by means of fast sweep signal system, described in [141] and depicted here in figure 5.22b. This device was composed by a signal generator, an inverter amplifier and a resistance connected in series with the Langmuir probe. This sweep system provided a sawtooth-like signal $V_{s g}$ whose frequency and symmetry can be modified by external controls. The current is measured by the voltage drop in the resistance $R_{d}\left(I_{p}=\Delta V_{d} / R_{d}\right)$. The probe voltage $V_{p}$ is calculated after the measuring process as

$$
V_{p}=\Delta V_{d}-V_{s g} G F+V_{r e f},
$$

where $G F$ is the gain of the inverter amplifier and $V_{r e f}$ is a reference voltage that allows to move the sweep signal to capture different parts of the complete I-V curve. This reference voltage can be easily modified before starting the measurement process. Even when this circuit is not unique and other options exist in the literature [137], this scheme has been tested in previous works $[99,100,141]$, providing good results and its verification or possible improvements are out of this thesis scope.

An analysis of the I-V curves obtained for signals with different symmetries and frequencies is now performed. In figure 5.23a, the three shapes studied in this section are shown. The rising one had a extended rising slope in time, whereas the falling one presented a longer falling slope. For the symmetric signal, both falling and rising flanks had the same length and therefore, this signal was divided in two edges denoted as rising $S E$ and falling SE. In the first two cases, we should remark that the signal's edge employed to obtain the corresponding I-V curve is only the larger one. The remaining 


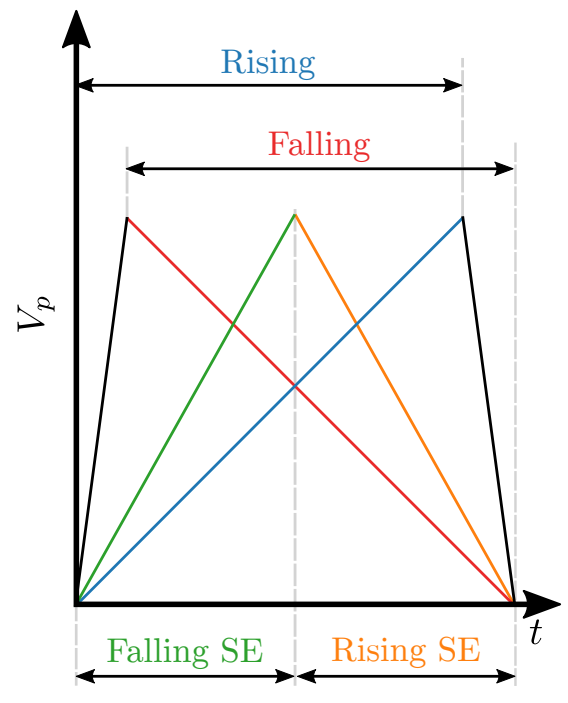

(a) Voltage shapes applied to the Langmuir probe

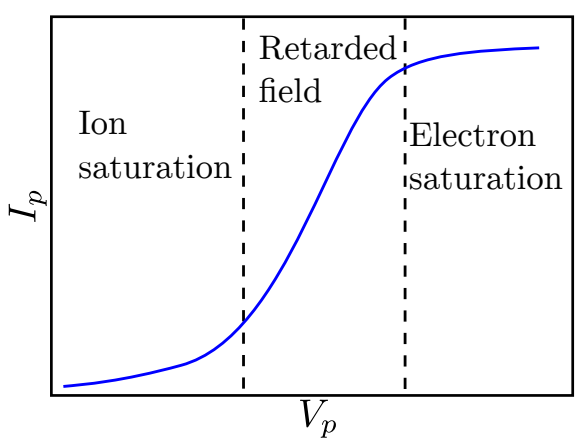

(b) Theoretical representation of an I- $V$ curve

Figure 5.23: (a) Voltage shape of the signals applied to the Langmuir probe as a function of time. (b) Theoretical representation of an I-V curve.

edge of the non-symmetrical signal should be large enough to allow the plasma recover the initial state, obtaining a periodical behaviour. The arrows of figure 5.23a indicate the measurement edges. Four I-V curves related to these four shapes are analysed.

To obtain an experimental I-V curve, a procedure as follows is done. First, an oscilloscope measures a number of cycles of the signal $V_{s g}$ and the voltage drop in the resistance $\Delta V_{d}$. Then, the longest edge of each signal is extracted (eliminating the perturbations created by the fast change of potential) and the I-V curve is obtained by means of the formulas presented above. In figure $5.23 \mathrm{~b}$, a theoretical representation of an I-V curve is drawn. It can be seen how the I-V curve separates into three zones. The left zone, when maximum ion current is captured and all electrons are repelled; the central zone, called retarded field; and the right zone where electron current is maximum and ions are repelled. In the experimental setup presented here, the reference voltage is used to capture only a part of this curve, related with the electron saturation zone. From this curve plasma parameters can be extracted by means of the intersection point of the fitting lines of two regions of the curve, the electron retarded field and the electron saturation current [131-133]. Properties of ions can also be extracted with a similar procedure from the study of the ion saturation. One of the first results found in the laboratory is that the obtained current is periodical, independently of the signal biased to the probe as long as it is periodical too and the plasma is stable. This is an important result because it allows us to choose any edge of the captured signal, ensuring similar plasma parameters. As seen below, this periodical behaviour is only obtained in our numerical simulations when charge-neutral interactions are accounted for. 


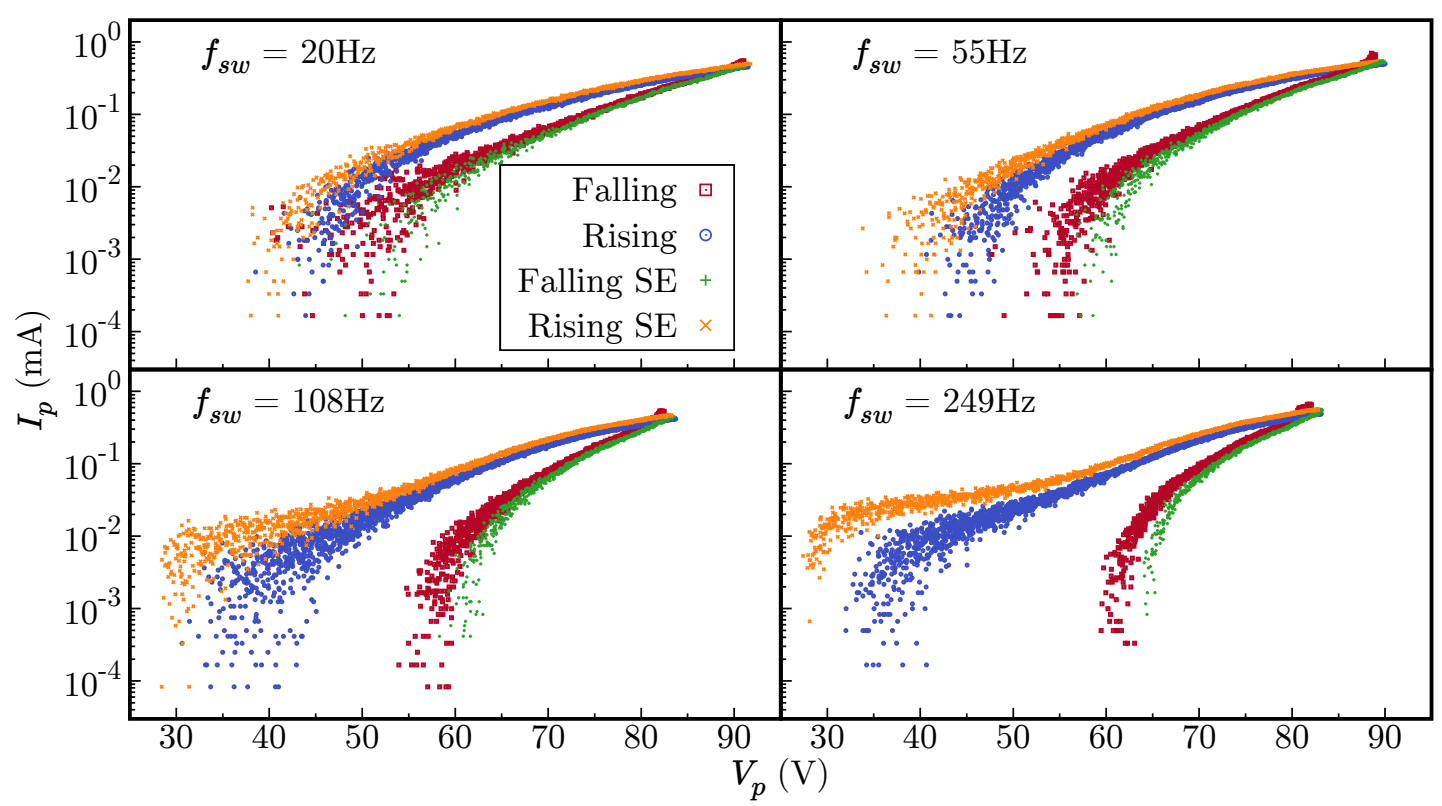

Figure 5.24: Experimental $I$ - $V$ curves of a collecting probe for signals at $f_{\text {sw }}=20,55,108$ and $249 \mathrm{~Hz}$.

The main results obtained by means of the setup described above are presented in figure 5.24, where the I-V curves for four different sweep frequencies $(20,55,108$ and $249 \mathrm{~Hz})$ are depicted. For the studied plasma, the electron and ion frequencies were in the range of $f_{p e}=(30-90) \mathrm{MHz}$ and $f_{p i}=(100-300) \mathrm{kHz}$, much higher than the sweep frequencies $\left(f_{s w} \ll f_{p i} \ll f_{p e}\right)$. As it can be seen, the four edges are grouped two by two. A first pair is constituted by the signals denoted as rising and rising SE, identified by an increasing applied voltage to the probe as the time evolves. The second pair is formed by the signals falling and falling $S E$, whose dependence on time shows a decreasing applied voltage. When sweep frequency is high $\left(f_{s w}=249 \mathrm{~Hz}\right)$ the two groups are separated and they can be clearly identified. On one hand, the falling pair is placed at more positive probe voltages $\left(V_{p}\right)$ than the other group, and the falling and falling $S E$ signals are very close one to the other and they almost coincide. Moreoever, the rising and rising $S E$ signals differ when frequency increases and they are shifted to more negative probe voltages with respect to the lowest frequency case. This behaviour is not substantiated for low frequencies, where the four signals are close and produce very similar plasma parameters. This separation is unlikely to be caused by a problem in the connection between the probe and the sweep system because the length of the lines is in the order of magnitude of meters and it is not enough to generate a capacitive effect that affects the probe biasing.

Figure 5.24 also shows how the separation of the two groups of I-V curves increase with the signal sweep frequency. For $f_{s w}=20 \mathrm{~Hz}$, the four I-V curves are very close one from the others and they almost coincide, providing similar plasma parameters. However, as the frequency increases, the curves separate from each other and for $f_{s w}=249 \mathrm{~Hz}$ the two groups of signals are clearly identified, being the falling group approximately 


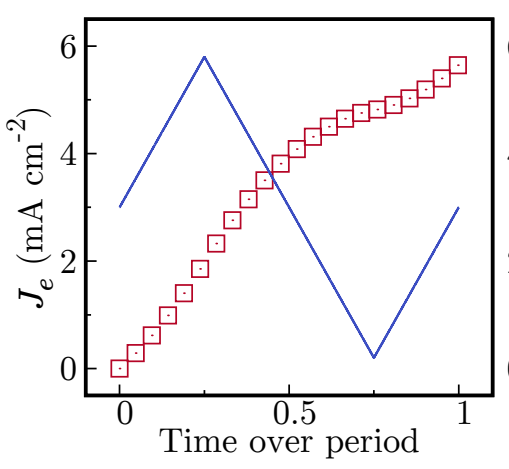

(a) Non-collisional

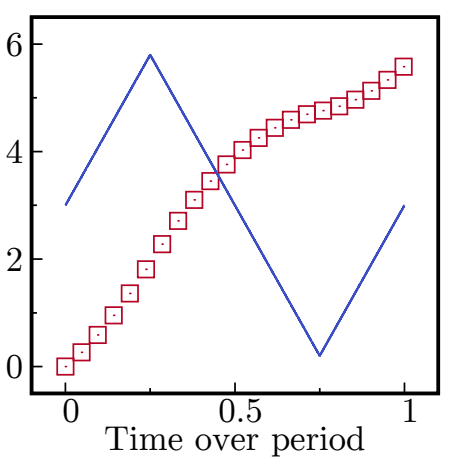

(b) No charge-neutral

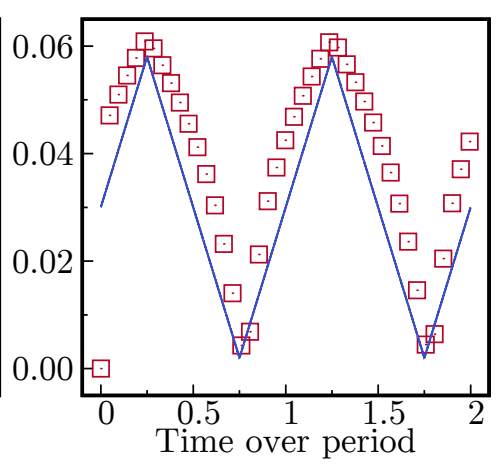

(c) Fully collisional

Figure 5.25: Time evolution of $J_{e}$ for (a) a non-collisional plasma, (b) a collisional plasma without neutrals and (c) the three species one-dimensional model.

20-30 V more positive than the rising group of signals. As stated above, the intersection between the electron saturation current and the retarded field regression lines provides the plasma potential. Therefore, the shifting observed in the I-V curves of figure 5.24 for the highest frequency provides different values for the plasma potential. Also, the group formed by the rising signals moves away from the classical shape of an I-V curve, which may hinders the right value of the regression lines, required to calculate the plasma parameters. Hence, the selection of one symmetry and frequency to obtain the right plasma parameters does not seem an easy task. Under ideal conditions, the local plasma potential should be very similar, regardless the signal employed to bias the probe. However, a strong dependency of shape and frequency was found. From an experimental point of view, a second measurement of the plasma potential can be alternatively obtained with the emissive probe by means of the floating potential method $[84,142]$ which should be operated close to the Langmuir probe. The crosscheck of the results should provide information to decide which appropriate symmetry of the signal should be used. Unfortunately, these results were not available during the development of this thesis, but they should be performed in the near future to obtain and additional source of information that helps us determine the right signal shape and frequency.

Nevertheless, it is reasonable to assume that the falling group yields the appropriate plasma parameters because they retain the typical shape of an I-V curve, specially for high frequencies. However, an independent explanation of this separation, not based on experimental techniques, to choose the right signal shape and frequency, is required.

\subsubsection{Numerical results}

The three species kinetic model presented in section 5.1 is solved with a time variable electric field that simulates the one generated by the potential difference between the probe and the quasi-neutral plasma. The limitation to only one space velocity of the model makes impossible the resolution of the coupled Poisson's equation and the wall 
effect. Nevertheless, the voltage signal can be translated into a time variable electric field if an electrostatic situation is assumed. For this propose, the following equations are used for the symmetric $\left(E_{s}\right)$, falling $\left(E_{f}\right)$ and rising $\left(E_{r}\right)$ signals

$$
\begin{aligned}
& E_{s}(t)=E_{0}+E_{v} \frac{2}{\pi} \arcsin \left(\sin \left(2 \pi t f_{s w}\right)\right) \\
& E_{f}(t)=E_{0} \begin{cases}-\frac{E_{v}}{0.89 / 2} t_{f} & \text { if } t_{f}<0.89 / 2 \\
+\frac{E_{v}}{0.11 / 2}\left(t_{f}-0.89 / 2\right)-E_{v} & \text { if } t_{f}<1.11 / 2 \\
-\frac{E_{v}}{0.89 / 2}\left(t_{f}-1.11 / 2\right)+E_{v} & \text { otherwise }\end{cases} \\
& E_{r}(t)=E_{0} \begin{cases}+\frac{E_{v}}{0.89 / 2} t_{f} & \text { if } t_{f}<0.89 / 2 \\
-\frac{E_{v}}{0.11 / 2}\left(t_{f}-0.89 / 2\right)+E_{v} & \text { if } t_{f}<1.11 / 2 \\
+\frac{E_{v}}{0.89 / 2}\left(t_{f}-1.11 / 2\right)-E_{v} & \text { otherwise }\end{cases}
\end{aligned}
$$

where $t_{f}=t f_{s w}-\left\lfloor t f_{s w}\right\rfloor, f_{s w}$ is the same sweep frequency used in the experimental problems and \lfloor\rfloor represents the floor function. These particular signal shapes are a direct translation of those employed in experiments, with the same proportion of short and long edges than $V_{s g}$. To simulate the sweep signal, the values $E_{0}=0.5 \mathrm{~V} / \mathrm{m}$ and $E_{v}=0.4 \mathrm{~V} / \mathrm{m}$ are employed. This sweep electric field provides energy to the plasma, which reaches a different energy state for each value of $E(t)$. This means that, at any value of $E(t)$ plasma has a different electron current density value, and a curve that relates both values can be obtained.

As was mentioned above, plasma has a periodical behaviour under any periodic signal biased to the probe. In this sense, the numerical simulations must also recover this periodical dynamics under any oscillatory electric field. To check if this behaviour is found in our model and the main reason of this behaviour, three different plasma models are analysed here under the effect of the periodical field $E_{s}(t)$ with $f_{s w}=249 \mathrm{~Hz}$. As explained in section 5.1.1, charge-neutral collisions have a huge influence in weakly ionized plasmas. Here, we explore if the collisional effects, specially charge-neutral, are important and required to explain the dynamics found in experimental results presented in the previous section. Figure 5.25 shows the evolution of $J_{e}$ under the influence of $E_{s}(t)$ for the same three cases as in section 5.1.1: non-collisional plasma, a collisional plasma without neutrals and the three species fully collisional plasma. We can see how no periodical behaviour is found and electron current increases with the always positive electric field if charge-neutral interactions are neglected. Moreover, chargecharge collective effects are not strong enough to limit the evolution of the species, but, when collisions between charge and neutrals are accounted for, a periodical behaviour appears, even if the electric field remains positive through the cycle. Although a periodical dynamics could be possible with an electric field of positive and negative values, it is not reasonable to assume that this can happen in front of a probe, as many effects determine the electric field shape. This means that the high density of neutral particles acts as a viscous media and sink of energy, providing a periodical behaviour of the charged species dynamics similar to the one found in the laboratory. Even when spatial effects can play a relevant role in the description of plasma in front of probes, 


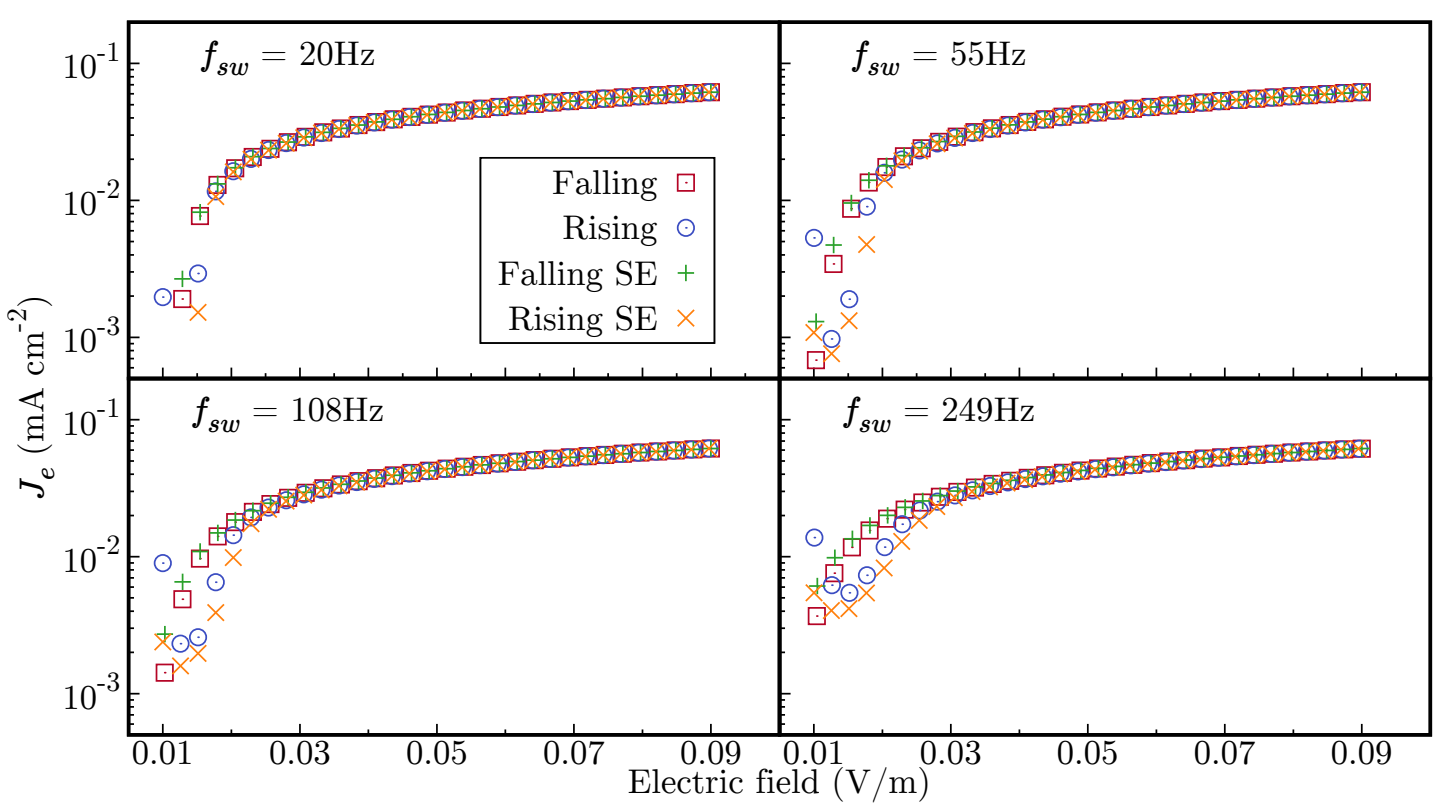

Figure 5.26: $J_{e}-E$ curves obtained with temperature dependent collision frequencies. A separation appears when $f_{\text {sw }}$ increases and edges group two by two.

it is clear that collision exchanges highly affect the WIP response to fast variations in the electric field.

Now, the $\mathrm{J}_{e}$-E curves obtained with the same shape and frequency as in the experimental problem are presented in figure 5.26. For low frequencies, the four edges produce a similar $\mathrm{J}_{e}$, but when the frequency increases, a separation between the falling and rising groups appears. This behaviour is similar to the one found in the experimental results, where the curves also split at higher frequencies, as seen in figure 5.24. In both, the experimental data and numerical results, falling edges remains close to each other and almost no change appears when the frequency increases. This indicates that falling edge symmetry is the best choice to bias a Langmuir probe as the numerical and experimental results agree. We saw in the experimental results how the falling edge high frequency produces a more defined I-V curve, specially in the retarding field, providing a more accurate interpolation of the curve's knee. In the numerical results, no difference among the falling edges for high frequencies seems to alter the shape of the $\mathrm{J}_{e}-\mathrm{E}$ curve and both shapes remain close to each other. This means that there is no reason to not use a relative high sweep frequency $>100 \mathrm{~Hz}$ if the falling symmetry is employed.

One important difference between both results is found: in the experimental data the knee of the rising edges shift to the left but in the numerical data, the rising edges move to the right. This may occur because the imposed external electric field in the numerical simulations does not properly reproduce the electric field generated by the charge separation close to a collecting probe.

To better analyse the different evolutions found, a zoomed view of the $\mathrm{J}_{e}$-E curves 

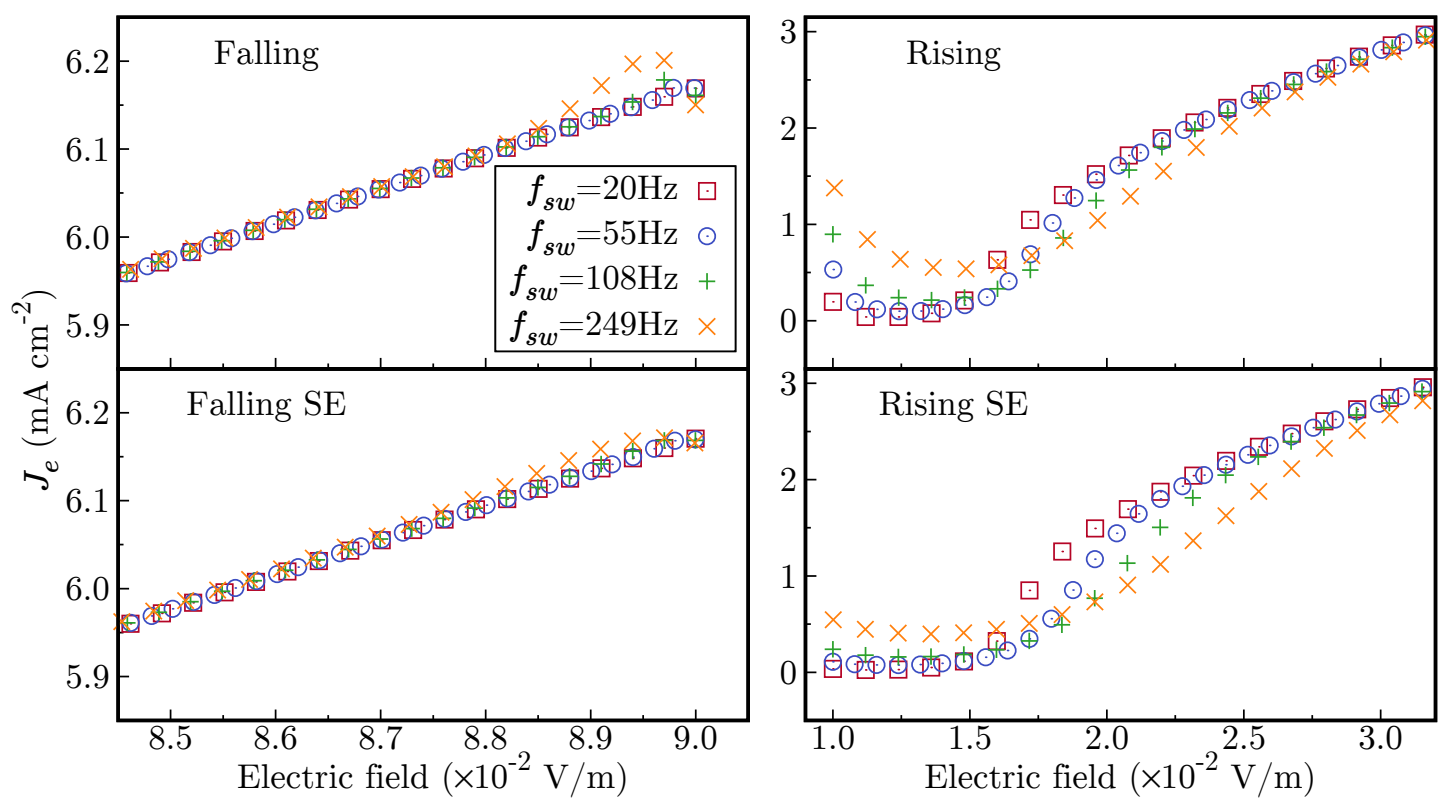

Figure 5.27: Zoomed view of the results in figure 5.26 corresponding to the starting point of the different measurement edges. Electrons reach an almost lineal velocity faster for the falling than for the rising signals.

from 5.26 is presented in figure 5.27. The point corresponding to the maximum (for the falling and falling SE) and minimum (for the rising and rising SE) value of the sweep signal is defined as the starting measurement point. This point indicates the start of the edge which is processed to obtain the $\mathrm{J}_{e}-\mathrm{E}$ or $\mathrm{I}-\mathrm{V}$ curves. An important difference in the relaxation times between the falling and rising edges is appreciated. For the falling edges (left) only small overshoots appear when the electric field changes. These jumps increase with the frequency but, due to the high electron temperature caused by the high electric field, an almost constant acceleration is rapidly recovered. On the other hand, electrons are cold when the rising signal changes and this produces a slow relaxation time. Also, this time increases with the sweep frequency, meaning that faster changes in the electric field create a larger perturbation in the system dynamics.

As in section 5.1.3, an analysis is performed now to check the influence of temperature dependent collision parameters in the system evolution. As seen in section 5.1.3, energy dependent collision parameters can modify the behaviour of a weakly ionized plasma when abrupt or fast changes in the electric field occur. Here, the goal is to analyse if this has some influence in the final shape of the $\mathrm{J}_{e}$-E curves, to check if this may be the cause of the curves separation. If collisions frequencies are set to a constant value, figure 5.28, no appreciable change appears as the sweep frequency increases and all symmetries produce almost the exact $\mathrm{J}_{e}-\mathrm{E}$ curve. We have seen in this work how the value of $E$ affects directly the electrons temperature, as presented in figure 5.8a, which influence the charge-neutral collision frequencies through the thermal velocity. If abrupt changes in the electric field appear, electrons recover faster an almost linear acceleration if they have a higher temperature. This dynamic is found exclusively if 
collision frequencies change with the species temperature through the simulation, i.e., if the model is solved in a self-consistent way, as performed in this chapter. But if collision frequencies do not change with energy, the rate of exchange between electrons and neutrals do not vary and a linear velocity is recovered faster, regardless the state of the electrons. This means that, if simplifications as constant collisions frequencies are employed, inconclusive or inadequate solutions may be reached, and no relation with the experimental results could be extracted. In figure 5.29, the starting point of the $\mathrm{J}_{e^{-}}$ E curves obtained with constant collision frequencies are presented for a better analysis of the electron response to the sharp electric field change. No difference appear between the falling and rising groups, which means that the relaxation times are identical for high and low electric field values. Nevertheless, a small difference among the signals when sweep frequency increases can be appreciated. This is caused because at high frequencies, plasma has less time to adapt to the electric field change. The difference among the curves is not big enough to produce an appreciable separation in the $\mathrm{J}_{e}-\mathrm{E}$ curves, but fast changes in the sweep signal may perturb the measurement process even in cases where collision parameters can be assumed as constant. The important result here is that relaxation times between falling and rising signals do not change with constant collision parameters, as it happens in the results presented in figure 5.26. This is, particles coming from a stable plasma into a collecting wall feel the neutrals through collisional process, which may vary depending on the acceleration applied to these particles by the electric field shape, which is influenced by the probe potential.

To continue with our analysis, the evolution of ions with variable collision parameters for the different signals presented in this section is studied. Even when this species has a lower density current than electron it is possible to study them with a Langmuir probe with the same procedure as electrons by just changing the reference potential until the part of the I-V curve corresponding to the ion saturation zone is captured [131]. The resulting ion current $J_{i}$ is pictured in figure 5.30 for temperature dependent collision parameters. It can be seen how for low frequencies, the curves keep a classical shape, but as frequency increases, ions do not recover the typical shape of an I-V curve, specially for the rising group. This is caused due to lower relaxation times of ions, which does not allow them to adapt fast enough to the changes in the electric field. This phenomena is important in the study of ion I-V curves, but can also affect the dynamics of electrons if a coupled Poisson's equation is solved where the ion dynamics close to the wall become relevant.

Numerical results show that the separation of the $\mathrm{J}_{e}$-E curves cannot be explained only with the use of different symmetries or frequencies. Energy dependent collision frequencies are required to obtain a similar qualitatively behaviour that those found in the experimental results. In the results presented in figure 5.24, the falling edges seem to look similar when frequency increases and also, the typical shape of an I-V curve is only maintained for this group. The rising signals are deformed for the highest frequencies and they show a strange shape, not identified as a typical I-V curve of a Langmuir probe. 


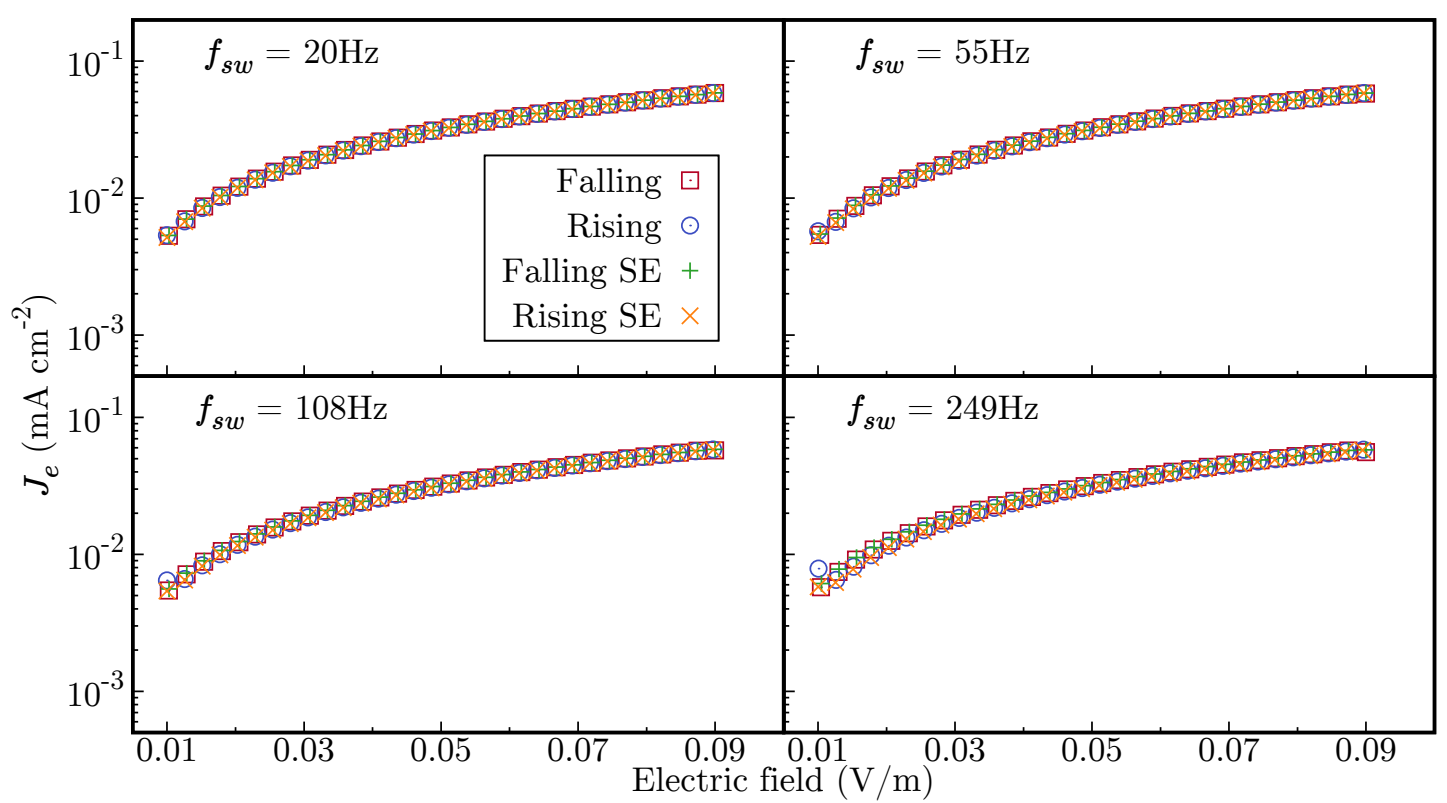

Figure 5.28: $\quad J_{e}-E$ curves obtained with constant collision frequencies. No appreciable difference appear in the curves no matter the frequency or symmetry applied.

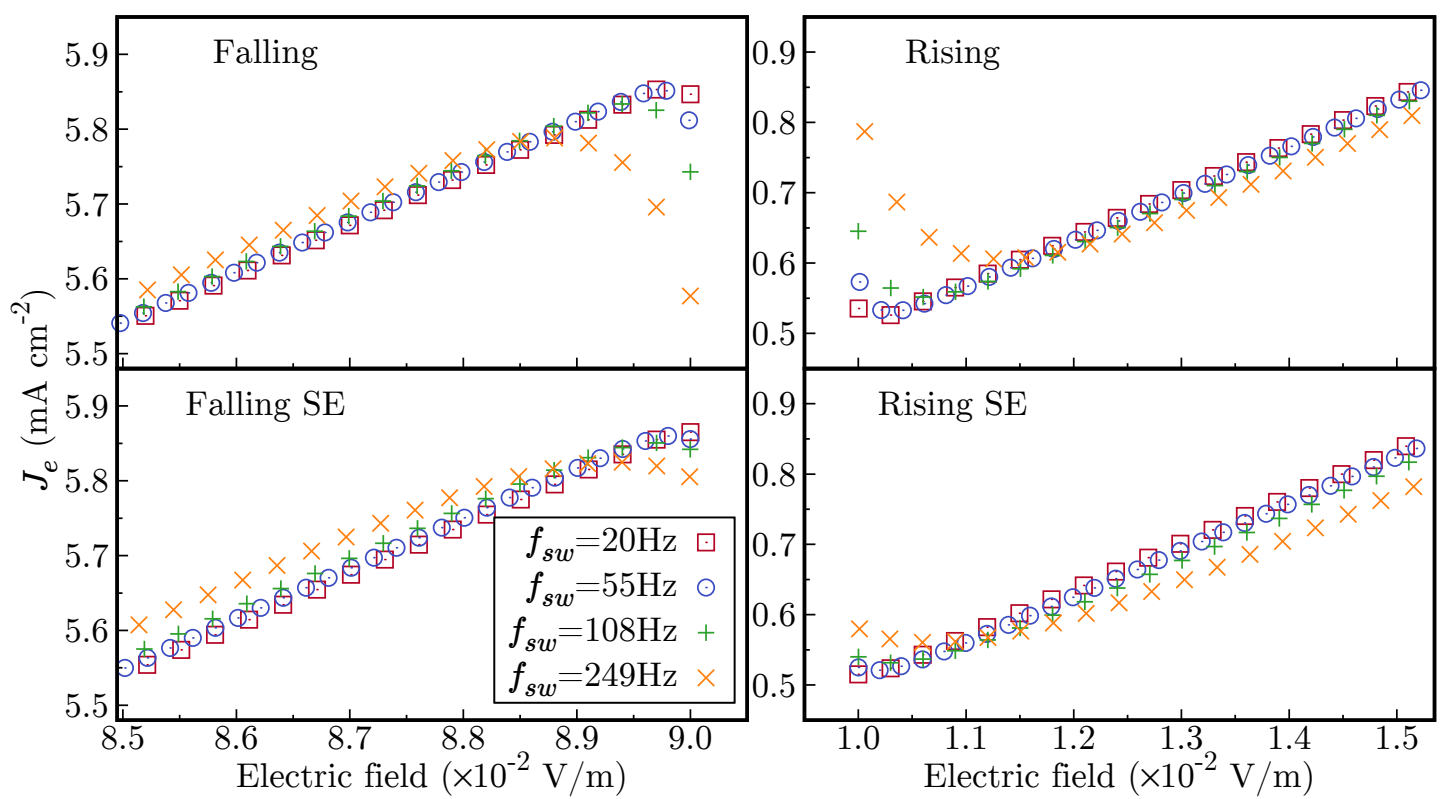

Figure 5.29: Zoomed view of the results in figure 5.28 corresponding to the starting point of the different measurement edges. 


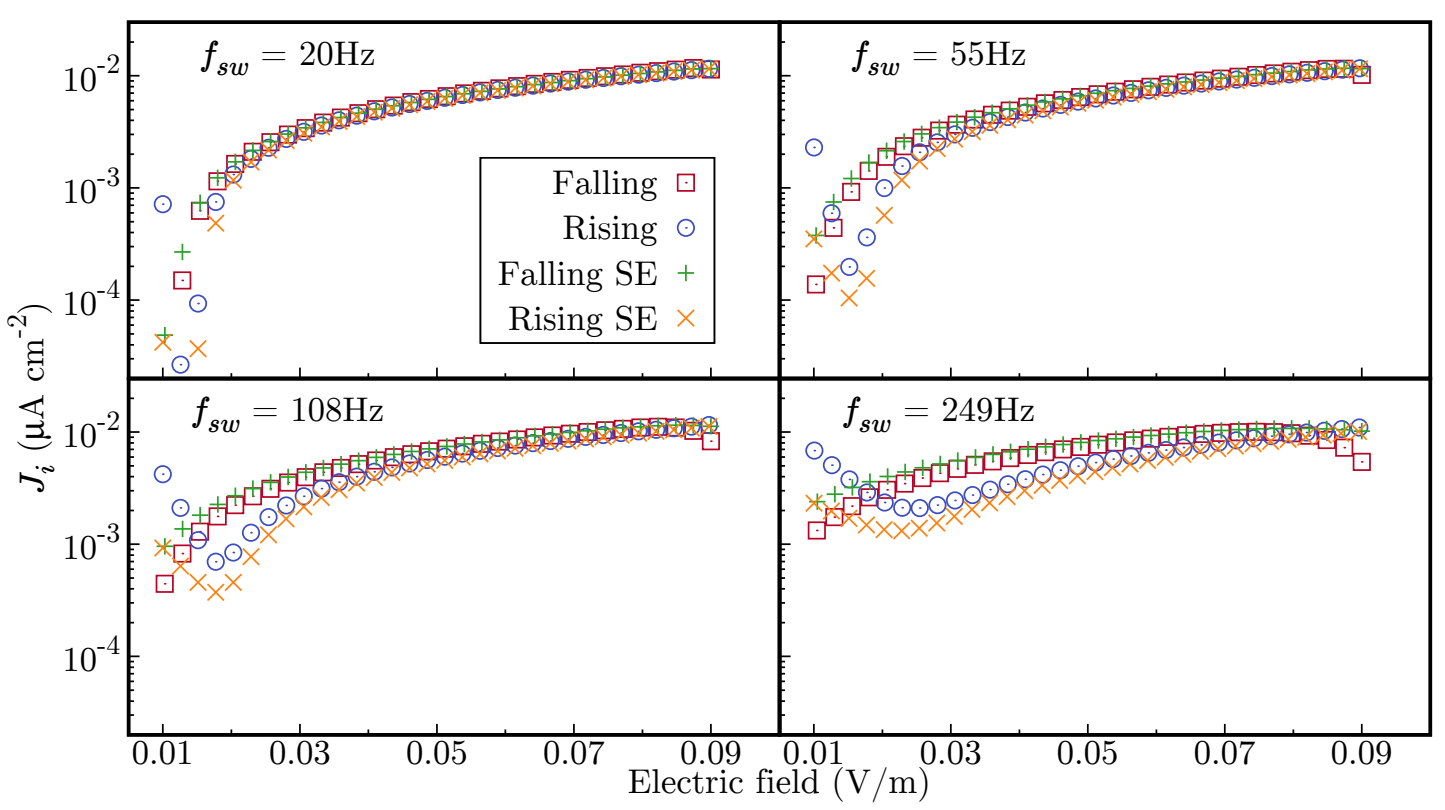

Figure 5.30: $J_{e}-E$ curves obtained with constant collision frequencies. No appreciable difference appear in the curves no matter the frequency or symmetry applied.

Neither the experimental I-V or the numerical $\mathrm{J}_{e}$-E curves are conclusive enough to choose a symmetry that provides the correct plasma parameters. Numerical data indicate that differences found in the I-V curves are related with the different ratios of exchange between electrons and neutrals. This means that electron-neutral interaction not only leads the dynamics of the system, but also the temperature dependency of the collision frequency is important to properly describe behaviours observed experimentally. Numerical results obtained in this work indicate that the best option for the sweep signal is the falling symmetry. This is based in the similar behaviours for the falling edges when frequency increases as well as in the fast recovery at the starting point due to the high electron-neutral interactions. Also, a relative high sweep frequency $(\sim 108 \mathrm{~Hz})$ seems to improve the experimental measurement without affecting the shape of the falling signals in the numerical and experimental results. Nevertheless, high frequency signals may perturb the evolution of ions, which require more time to adapt due to their high mass and low temperature and may end up influencing the electron dynamics.

The numerical and experimental results seem to agree with the conclusions about the correct shape of the sweep signal. However, an alternative measurement to cross-check the results and find a conclusive experimental support is needed. For this task, an emissive probe could be employed by means of the floating potential method. In this method no biasing signal is involved, and a second independently measurement of the plasma potential could be obtained. This would allow to cross-check the parameters provided by the I-V curves of the collecting probe and find some conclusive decision about the signal shape and frequency. 


\subsection{Transport coefficients for electrons in WIP}

Transport coefficients define how a mass and energy transfers evlve under to variations in the electromagnetic field or some magnitude gradients. These are necessary, for example, to close the hierarchy of fluid equations. Many parameters can influence the value of transport coefficients, as density or temperature. To obtain transport coefficients, collisional effects must be accounted for as an steady situation has to be reached to obtain a time-independent solution.

In this section, we focus in the computation of electron temperature, density current and heat flux as a function of the electric field and ionization ratio in an argon plasma. As seen in section 5.1.4, the relation between charges and neutral density highly influence the plasma dynamics. Other effects commonly employed in the calculation of transport coefficients, as the gradient of temperature, are neglected, yet they can be easily included as non-homogeneous terms $[42,43]$. To obtain accurate descriptions of transport coefficients, a space composed by the directions perpendicular and parallel to the electric field is accounted for. To represent the collisional processes, two-velocity terms presented in chapter 4 are used. The charge-charge interaction is described by the Fokker-Planck - Landau operator, while charge-neutral interactions are modelised by a Dougherty term. This last term is chosen for similitude with the one-dimensional results presented in this chapter and by the constant value of the diffusive terms, which simplify the numerical implementation when high ratios of mass, density and temperature appear.

\begin{tabular}{lcll}
\hline & Mass $(\mathrm{g})$ & Temperature $(\mathrm{eV})$ & Density $\left(\mathrm{cm}^{-3}\right)$ \\
\hline $\mathrm{e}$ & $9.10938356 \times 10^{-28}$ & 1 & $r_{i} \frac{n_{T}}{2}$ \\
$\mathrm{i}$ & $72819.6 \times m_{e}$ & $5 \cdot 10^{-2}$ & $n_{e}$ \\
0 & $m_{e}+m_{i}$ & $5 \cdot 10^{-2}$ & $n_{T}\left(1-r_{i}\right)$ \\
\hline
\end{tabular}

Table 5.2: Argon plasma to compute transport coefficients by a two velocity collisional model.

An argon plasma, of properties presented in table 5.2, is employed here. Due to numerical constrains, only electrons are advanced in time and ions and neutrals are left fixed, as previous result have shown that the light species is the one that reacts the most to the electric field. The total density is $n_{T}=10^{-13} \mathrm{~cm}^{-3}$ and the electric field takes the values $0.1,0.2$ and $0.3 \mathrm{~V} / \mathrm{m}$, as usual in this chapter.

The following collision operators are employed for each exchange type as

$$
l^{e \alpha}=8 \pi \frac{4 \pi q_{e}^{2} q_{\alpha}^{2} \lambda^{e \alpha}}{m_{e}^{2}}, \mu^{e 0}=\sigma^{e 0} V_{t h}^{e}
$$

where $\alpha=e, i$ to account for self-collisions and the interaction with ions and $V_{t h}^{e}=$ $\sqrt{\frac{3 k T_{e}}{m_{e}}}$ is the thermal velocity in a three-dimensional space. The Coulomb's Logarithm is computed following the classical expressions from [129], presented in equations (5.9) and (5.10). Electron neutral cross-section equals $10^{-16} \mathrm{~cm}^{2}$ and is constant. 


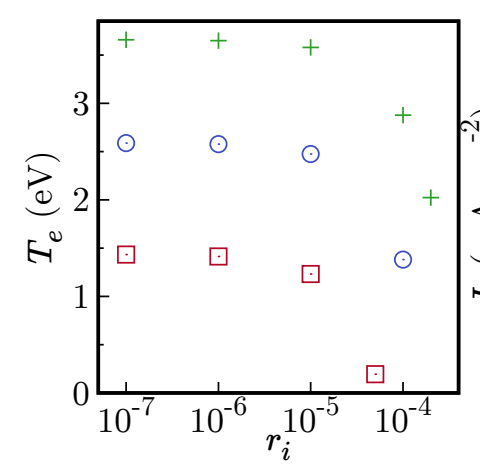

(a) Temperature

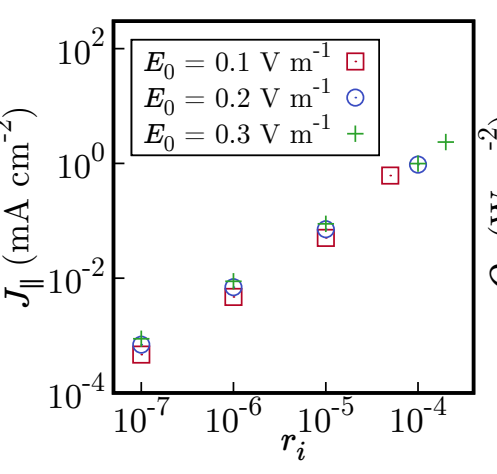

(b) Density current

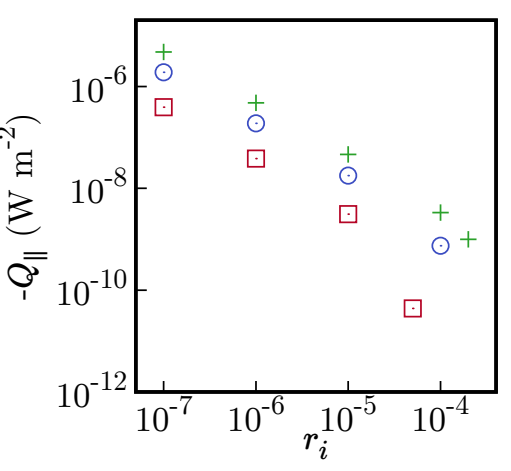

(c) Heat flux

Figure 5.31: Electron temperature, density current and heat flux in the parallel direction as a function of the electric field and ionization ratio. Simulation time after 4000 iterations correspond to $\sim 0.9 \mathrm{~ms}$

Three main parameters are analysed here, the electron temperature $T_{e}$, density current $\mathbf{J}=\left[q_{e}\right]$ and heat flux $\mathbf{Q}=\left[v^{2}\right] / 2 n_{e}$. We focus our analysis on the parallel direction, as is the one in which the electric field acts. As usual, in absence of temperature and density gradients, fluxes are assumed to obey

$$
J_{\|}=K_{e} E, \quad Q_{\|}=K_{T} E,
$$

where subscript $\|$ determines the direction and $K_{e}\left(E, r_{i}\right)$ and $K_{T}\left(E, r_{i}\right)$ are two unknown functions that relates the electric field with the electron density current and heat flux, respectively, in connection to the usual electrical and thermal conductivities.

Figure 5.31 pictures the main results of this analysis for the two relevant parameters defined here. Temperature remains constant for $r_{i} \lesssim 10^{-5}$, but drops rapidly afterwards. Moreover, an increment in the plasma temperature as a consequence of more intense electric fields also appears. A linear behaviour is found for density current and heat transfer up to a certain ionization ratio, as it happened in the one dimensional case. Moreover, the linear behaviour is hold for higher $r_{i}$ as $E$ increases. Heat flux has a linear decrement with $r_{i}$ linearly up to a certain value of $r_{i}$ where its starts quickly decreasing. The interpolation of $K_{e}$ and $K_{T}$ is not an easy task, as many parameters influence them and not always linearly. However, these simulations indicate that weakly ionized plasmas behave linearly with the ionization ratio up to a certain point in which charge-charge exchange become the relevant phenomena and plasma is closer to a fully ionized description. As it happened in the one-dimensional results, the limits of this linear zone are not easy to clarify, as many factors influence it. However, a significant difference with the dynamics found at high ionization ratios respect to the one-dimensional model is found. As $r_{i}$ increases, fluxes seems to hold or even increase. This can be caused, e.g., by the stationary assumption employed for heavy species and requires a further analysis.

The calculation of transport coefficients by means of self-consistent simulations accounting for collisional effects is of major importance for the characterization of plas- 
mas. Dynamics found in previous simulations with the one-dimensional three species kinetic model are recover here as well, meaning that the lost of linear response with $r_{i}$ is not a numerical effect or it is caused by the model limitations, but it is a physical response of the plasma that indicates a change in its behaviour as $e-i$ exchange becomes a relevant phenomenon. Nevertheless, the resolution of the complete distribution function in a physically meaningful way allows an easy computation of fluxes by just integrating it in the velocity space, which ease the calculation of all transport coefficients.

\subsection{Conclusions}

In this chapter, the progress performed in this dissertation to deal with the kinetic description of Weakly Ionized Plasmas has been presented. The main output is the one-dimensional three species kinetic model, that allows a fast computation to describe the dynamics of three species (electrons, ions and neutrals) under the effect of an intense electric field that leads the system evolution in one privileged direction, if space homogeneity is assumed. Even though our focus has been the description of an argon plasma, other types could also be described by just extending the model for the new species and collisional terms,. This model is useful to obtain a dynamics that includes all elastic exchanges among species and check their effect in a large variety of scenarios. For example, differences due to the energy dependency of the collision frequencies have been found, specially in transitory situations, e.g., the variation of the relaxation times or overshoots appearing when the electric field rapidly changes. The effect of the ionization ratio was also analysed, finding a non-linear plasma response that depends on the electric field intensity and total plasma density.

Moreover, the model has been employed to analyse discrepancies found when a Langmuir probe is biased with different voltage signals to characterize a plasma. Simulations suggest that these changes are caused by variations in the exchange rates between charged and neutral particles. Numerical and experimental results agree on the shape and frequency that must be employed to bias a Langmuir probe. However, further analysis and independent data is required to provide a better understanding, as an independent measure of the plasma potential by means of the floating potential method or a numerical simulation that accounts for the self-consistent electric field and wall effect.

Besides this, the computation of transport coefficients for the electrons in presence of ions and neutrals has been carried out. Although these are still preliminary results, the capability of our model to include complex collision operators, as the Fokker-PlanckLandau, and solve the complete distribution function far from equilibrium situations opens a wide range of possibilities to obtain transport coefficients, e.g., in non-thermal equilibrium scenarios, where other methods, as those based on perturbation or linearisations, may fail. Nevertheless, the work performed here must be continued to 
improve these calculations. However, behaviours related with high ionization ratios found in one-dimensional simulations appear also in here, meaning that the change in the plasma dynamics is not related with the limitations of the one-dimensional model, but it is a physical response. Electron-ion collisions becomes the dominant collisional process, making electrons exchange a large amount of energy to this heavy species and resulting in a complete different steady state, as a higher electric field is required to extend the linear response zone. This could lead to a new path to define the limits of WIP and study the transition towards fully ionized plasma. 


\section{CHAPTER 6}

\section{PLASMA-WALL INTERACTION}

The plasma-wall interaction is still an open problem of mayor importance in Plasma Physics. Dynamics of light and heavy species close to a wall affects measurement processes, service life of walls and coatings and surface treatment processes. This interaction appears in experiments carried out involving plasmas $[83,143]$ and also, in vessels flying in space [86]. Many works have provided fluid models, as the those by T. Gyergyek et al. $[116,144]$ or kinetic descriptions, as the works by M. D.Campanell et al. $[89,145]$ or J. P.Sheehan et al. $[98,143]$, as well as many other authors $[23,85,146-150]$. Moreover, different approaches to describe the emission of electrons as the works by J. L. Domenech-Garret et al. $[88,113]$ and references therein, can be found in the literature, but the description of the complete processes involved in the plasma-wall interaction are still an open topic of discussion because of the variety and peculiarities of each plasma device.

An important case of plasma-wall interaction appears in the characterization of plasmas, usually carried out with emissive $[84,142,151]$ or collecting probes [134,135,137], to calculate plasma parameters such as temperature, density or plasma potential responsible of energy transference. The modelisation of this interaction is usually established by models that may not capture all microscopic processes involved, specially in Weakly Ionized Plasmas (WIP) for which the inclusion of a dense heavy cold neutral species hinders the development of models [137-139]. Our research group is interested in the use of these probes to analyse the plasma generated in our low temperature laboratory $[99,100,152]$, making this topic of main interest.

In this chapter, the progress performed through this work to analyse the evolution of Weakly Ionized Plasmas close to emissive or collecting walls is presented. Firstly, we focus in the electron emission into a vacuum chamber by a hot planar infinite wall, 
modelised as a discontinuous media. The influence on the emitted density current of the self-consistent electric field and the electron-neutral interaction is analysed. Different electric potentials are applied to the wall, to check if a saturation current is reached, as it happens in experimental results [83,140]. Secondly, the collection of electrons and ions coming from a quasi-neutral weakly ionized plasma is studied. Influence of the heavy species dynamics and charge-neutral interaction are investigated.

\subsection{Emissive wall as a discontinuous plasma medium}

The thermionic emission of electrons is covered in this section. This is usually modelled as a boundary value problem from both fluid $[116,150]$ and kinetic $[98,149]$ approaches. By heating up a metallic wall or wire, electrons are ejected from the metal. By biasing the emissive probe at different potentials, a curve that relates the emitted current and the wall voltage can be obtained. The emitted current reaches a maximum when the potential difference between the emissive element and the vacuum chamber is such that becomes stationary. This current is also known as saturation current.

This section offers a deeper look inside the work [26], where the Propagator Integral Method was employed in a $1 \mathrm{D} 1 \mathrm{~V}$ phase space to solve a discontinuous system. Here, a piecewise kinetic model is presented to describe the transition between a hot metallic wall and a vacuum chamber filled with a neutral gas. The problem is formulated as an heterogeneous plasma in 1D1V phase space in planar geometry. The model describes the dynamics of electrons in the metal (for $x<0$ ) and in the vacuum chamber (for $x \geq$ 0 ) from a pure kinetic point of view. Inside the metallic wall, a phenomenological driftdiffusion operator with commonly employed quantum correction relaxes the electronic ensemble to keep them at the wall temperature and thermal velocity corresponding to it, whereas elastic electron-neutral collisions and the effect of the self-consistent electric field are accounted for in the vacuum chamber. A physical interface arises naturally to continuously connect both zones up to a specific scale. In the outer zone, electrons are affected only by the wall or the vacuum chamber phenomena. The set of equations that describes the electron distribution function evolution is defined as

$$
\begin{aligned}
& \frac{\partial f_{e}}{\partial t}+v \frac{\partial f_{e}}{\partial x}+\frac{q_{e} E}{m_{e}} \frac{\partial f_{e}}{\partial v}= \\
& \begin{cases}-\frac{\partial}{\partial v}\left\{-\nu_{w}\left(v-V_{t h}^{w}\right)\left(1-\Theta f_{e}\right)-\frac{\nu_{w}}{2} V_{t h}^{w 2} \frac{\partial}{\partial v}\right\} f_{e} & \text { if } L_{-} \leq x<0 \\
-\frac{\partial}{\partial v}\left\{-\nu^{e 0}\left(v-u_{0}\right)-\frac{\nu^{e 0}}{2}\left(E_{e}+E_{0}-2 u_{e} u_{0}\right) \frac{\partial}{\partial v}\right\} f_{e} & \text { if } 0 \leq x \leq L_{+}\end{cases} \\
& \frac{\partial^{2} \phi}{\partial x^{2}}=\frac{1}{\epsilon_{0}} q_{e} n_{e} \text { if } 0 \leq x \leq L_{+},
\end{aligned}
$$

where $f_{e}=f_{e}(x, v, t)$ is the electron distribution function, $q_{e}$ and $m_{e}$ are the electron charge and mass, $E(x, t)=-\partial \phi / \partial x$ is the electric field, $\phi$ is the potential, $L_{ \pm}$are the edges of the numerical grid in the $\mathrm{x}$ direction, $V_{t h}^{w}=\sqrt{k T_{w} / m_{e}}$ is the thermal velocity of the electrons at the wall, $T_{w}$ is the wall temperature, $\nu_{w}$ and $\nu^{e 0}=n_{0} \mu^{e 0}$ are the 
collision frequencies for the electrons in the wall and the electron-neutral collision term respectively and $\Theta=h / 2 m_{e} n_{e}$ where $h$ is the Planck constant, is a parameter related to gas quantum degeneracy effects [153, Section 17.5]. Here, $n_{0}$ is the neutral density in the vacuum chamber, related with the background pressure, $E_{0}=k T_{0} / 2 m_{0}+u_{0}^{2} / 2$ and $u_{0}$ are the energy and velocity of the neutral background gas in the vacuum chamber and $\epsilon_{0}$ is the permittivity in vacuum. In this section, $u_{0}=0$ to represent a fixed density of neutrals. The Poisson's equation for the electric potential is solved with Dirichlet boundary conditions at $x=0$ and at the end of the numerical mesh $x=L_{+}$. For $x<0$, an uniform potential is assumed, i.e., no electric field inside the metal.

The evolution of the system resembles a Brownian motion in all the domain with a quantum correction term, $1-\Theta f_{e}$, for the metallic wall and an external force, the electric field, for $x \geq 0$ [26]. Inside the wall the drift-diffusive operator acts over the electrons to leave the mean velocity and temperature to the initial one, $V_{t h}^{w}$ and $T_{w}$ respectively. The possible ions and neutrals in the metal are fixed and do not interact with the electrons, i.e., they do not influence at all the electron dynamics for $x<0$. When electrons jump into the vacuum chamber, they collide with the neutral background and are affected by the self-consistent electric field. Electron-electron interaction is neglected due to the low density of particles, but could be included as an additional collision term [25]. Ionization of neutral particles is neglected due to the short-time scales analysed.

A widely extended mechanism to emit electrons into a vacuum chamber is by heating up a metallic wall or wire by an external current, also known as thermionic emission. To modelise the emission of a hot metallic wall into a vacuum chamber with the discontinuous model presented above, an initial amount of particles is set on the metallic region, following the Richardson-Dushman law [154,155], which is usually expressed as

$$
J_{r d}\left(T_{w}\right)=C T_{w}^{2} \exp \left(-\frac{e W_{f}}{k T_{w}}\right)
$$

where $C$ is the Richardson constant, $W_{f}$ is the work function of the metal, $e=\left|q_{e}\right|$ and $J_{r d}=e n_{e} u_{e}$ is the surface density current emitted by the probe, where $n_{e}$ and $u_{e}$ are the electron norm and mean velocity. To match the material used commonly in the emissive probes (tungsten), $C=6.02 \cdot 10^{5} \mathrm{~A} / \mathrm{m}^{2} \mathrm{~K}^{2}$ and $W_{f}=4.55 \mathrm{eV}$ [156]. If the thermal velocity is assumed for the electrons in the metal $\left(u_{e}(x<0, t=0)=V_{t h}^{w}\right)[152]$, its density can be extracted from equation (6.3) as

$$
n_{e}(x<0, t=0)=\frac{J_{r d}\left(T_{w}\right)}{e V_{t h}^{w}},
$$

which only depends on the probe properties and temperature. These parameters are cast into a drift-Maxwellian distribution function to act as initial condition in the 1D1V problem. The drift-diffusive operator for $x<0$ keeps constant these macroscopic properties through the simulation, except close to the discontinuity, where the influence of the vacuum chamber can affect electrons inside the metal, which require time and space length to reach the wall conditions. 
Numerically, equation (6.2) is solved at each time step with Dirichlet boundary conditions at $x=0$ and $x=L_{+}$as in section 2.4.1. Equation (6.1) is solved with the basic integral advancing scheme with homogeneous boundary conditions and without nonhomogeneous term for a $1 \mathrm{D} 1 \mathrm{~V}$ phase-space, as presented in section 2.2.2. The problem is fed at the numerical equivalent of $-\infty$, i.e., at $x=L_{-}$in the numerical problem, to maintain a flux of electrons with the metal temperature and corresponding thermal velocity. At $x \rightarrow \infty$, i.e., the end of the numerical grid for the positive side, $x=L+$, the solution is left free to ensure that the electrons that may reach this situation does not perturb the distribution function close to the wall.

Due to numerical limitations required to properly represent all the disparate phenomena, time and length scales employed must be very small, $\sim 10 \mathrm{ps}$ and $\sim 10 \mu \mathrm{m}$ respectively, which impose a high collisional rate between electrons and neutrals to ensure that the lowest diffusive scales are properly represented. Moreover, the large peak of electrons created close to $x=0$ induces a huge charge separation, which results in an intense electric field that can perturb the simulations where real collisional scales are employed. This could be improved following two paths: solving the problem in a cylindrical geometry, and accounting for a continuous description of the electric potential that accounts for the electrons inside the metal. For this problem, reference density and cross section are $10^{20} \mathrm{~m}^{-3}$ and $10^{-15} \mathrm{~m}^{2}$, respectively. The characteristic length is the mean free path of the characteristic density and cross sections, defined as $L_{c}=\left(n_{c} \sigma_{c}\right)^{-1}=10^{-5} \mathrm{~m}$. The non-dimensional electron-neutral collision parameter is chosen as $\mu^{e 0}=1$, to ensure that the numerical problem is stable and the $x-x$ and $x-v$ diffusion are correctly represented. A similar behaviour to the one presented in the following section is expected to be found if the computational resources to represent the proper scales and length were available. The collisional parameter at the wall, $\nu_{w}$ is set to 10 to represent the fast relaxation that suffers the electrons at the metallic wall to recover the initial temperature and mean velocity. Nevertheless, a case with realistic collisional scales and without electric field is presented in section 6.1.3 to ensure that the background pressure influences the wall emission. The parameter $\Theta$ equals one in non-dimensional units, yet its definition should be improved in future works.

For these computations, $\tau=0.1$ and a grid of $\Delta x=1 / 30$ and $\Delta v=0.2$ with 300 and 100 points in the $x$ and $v$ directions respectively are used. These values may produce an unstable time evolution in other numerical methods, but PIM deals with this coarse grid, providing smooth, conservative and realistic time evolving solutions. The grid covers the distance from $x=L_{-}=-3$ up to $x=L_{+}=7$ and it is centred in $v=0$. A convergence criteria to cut-off the iterative process is utilised. In the simulation, the evolution of the electron flux at $x=0$ is studied, and computation stops when the relative difference between two consecutive iterations is less than $0.1 \%$. To ensure that an almost steady situation is reached in all scenarios and that any possible perturbation due to the discontinuous initial conditions is dumped, a minimum of 500 iterations is computed for each case. The analysis of this convergence criteria is presented in appendix 


\subsubsection{Influence of background pressure for no potential difference sce- nario}

A first set of results is presented for the case of no potential difference between the wall and the vacuum chambers, i.e., both $\phi$ at the boundaries are set to zero.. A sweep is performed with two parameters: the wall temperature $T_{w}$ and the neutral background pressure $p_{0}$. Wall temperature varies in the range $2000-2300 \mathrm{~K}$ with increments of $25 \mathrm{~K}$, and neutral pressure equals 1, 2, 3, 4 and $5 \mathrm{~Pa}$ with a fix temperature of $300 \mathrm{~K}$. Assuming a perfect argon gas model, the neutral density is obtained with these two parameters, which influence the electron-neutral collisional process through the frequency $\nu^{e 0}=n_{0} \mu^{e 0}$. The total density current, defined as $J_{T}=[e]$, at two positions is shown in figure 6.1. Total density current remains small up to $T_{w}=2100 \mathrm{~K}$, where the exponential behaviour of equation (6.4) translates into an increment of the emitted electrons. Density current at $x=0$ increases with $p_{0}$ up to $3 \mathrm{~Pa}$, when it reaches a maximum, and decreases for higher pressures. Thence, a non-monotonic behaviour with the background pressure is found. This is caused by the interplay among relatively high energy emitted electrons, the self-consistent electric field that appears and the effect of collisions. It is important to remark that the electric field is related with the density of electrons, equation (6.2), as emitted electrons deviate $n_{e}$ from 0 , creating a non-uniform electric potential. For intermediate pressures, collisions slightly reduce the amount of electrons close to the discontinuity, which increases the electric field nearby $x=0$, resulting in a large amount of electrons constrained close to the wall. However, as pressure increases, electron-neutral interaction gains relevance, a fact that highly reduces the current density at $x=0$.

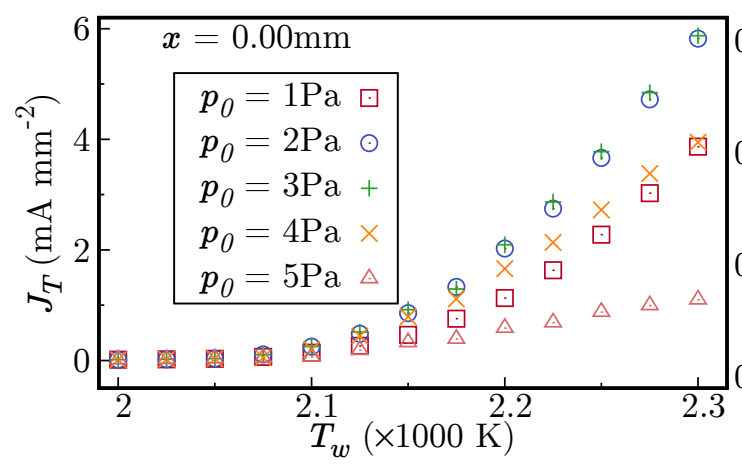

(a)

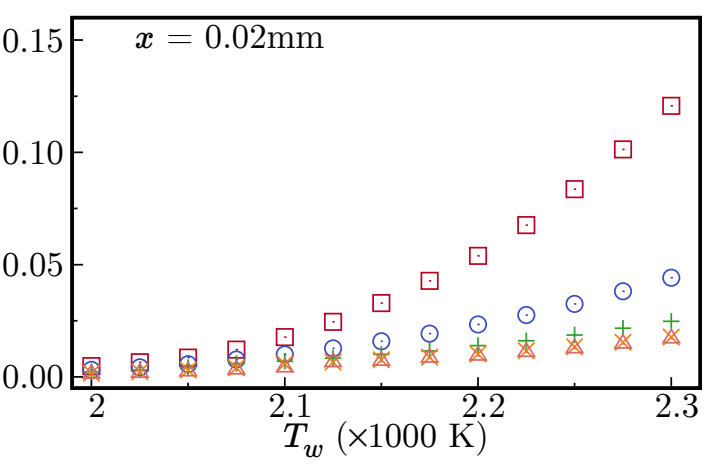

(b)

Figure 6.1: Emitted current at (a) $x=0$ and (b) $x=0.02 \mathrm{~mm}$. Non-monotonic situations arise close to the wall.

Far from the wall, figure $6.1 \mathrm{~b}$, the emitted current lessens for higher pressures. This means that a detector positioned far from the emissive wall always detects a lower electron current as pressure increases. A similar reduction in the emitted current has been found experimentally, for example in [140, Section 4.1], where measurements of the current emitted by a tungsten wire into a vacuum chamber were performed. Figure 6.2 resumes some of the results presented in the work cited above. It can be seen how 


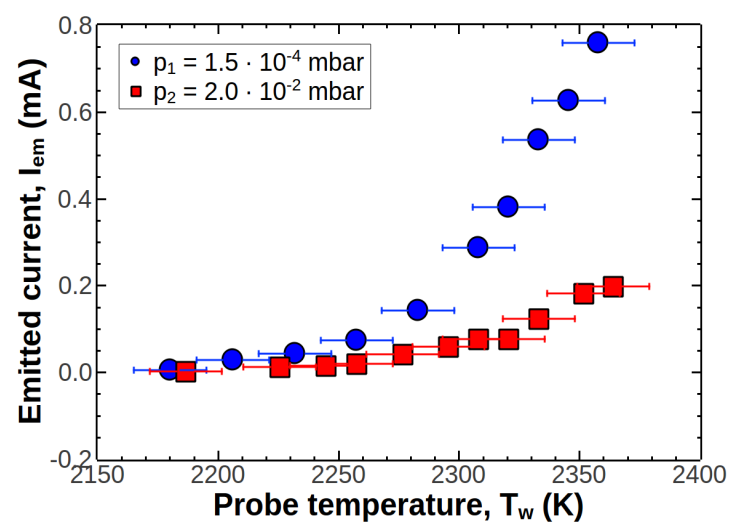

Figure 6.2: Experimental measures of the current emitted by a wire at two different background pressures. Figure taken from [140, Section 4.1.2] with full permissions from S. P. Tierno.

the current emitted by an emissive probe reduces by the presence of a higher neutral background pressure. In both, the experimental and numerical results, the background pressure reduces the emitted current. These similar qualitatively behaviours indicate that the emission of electrons into a vacuum chamber is influenced by the presence of a neutral background gas.

For a better comprehension of the dynamics of the heterogeneous media described by equation (6.1), the distribution function $f_{e}$ is analysed. It is important to be aware that, thank to the capability of the PIM to describe drift-diffusive problems with discontinuous piecewise coefficients, the complete distribution function is computed, enabling the analyse of electrons dynamics without using perturbation methods or other linear approximations.

To begin this analysis, contours of $f_{e}$ at $2000 \mathrm{~K}$ are presented in figure 6.3. The increment in background pressure has two main effects over the emitted electrons: it helps to thermalize the distribution function in the vacuum chamber, which become closer to a Maxwellian, and it retains electrons nearer to the wall increasing the population in the surroundings of $x=0$. This fact means an increment of the $f_{e}$ peak, and a reduction of the amount of electrons able to travel far from the wall, diminishing the emitted density current. Inside the modelled wall, $x<0$, the electrons deviate from the initial situation for a very short length scale, meaning that they are rapidly thermalized to the wall conditions thanks to the drift-diffusive operator. The variation of electron density caused by collisional effects also influences the electric field.

The steady contours for $T_{w}=2300 \mathrm{~K}$ are studied in figure 6.4 , and a change in the behaviour of the distribution function respect to the previous case is found. Here, a doubled peaked distribution function is clearly identified for the low pressures presented. This is caused by the strong electric field generated by the emitted electrons, resulting in a large portion of them returning and being collected. This strong electric field generates a second peak of particles with negative mean velocity. However, as pressure increases, collisions become more relevant and the two peaks fuse into one, resulting in 


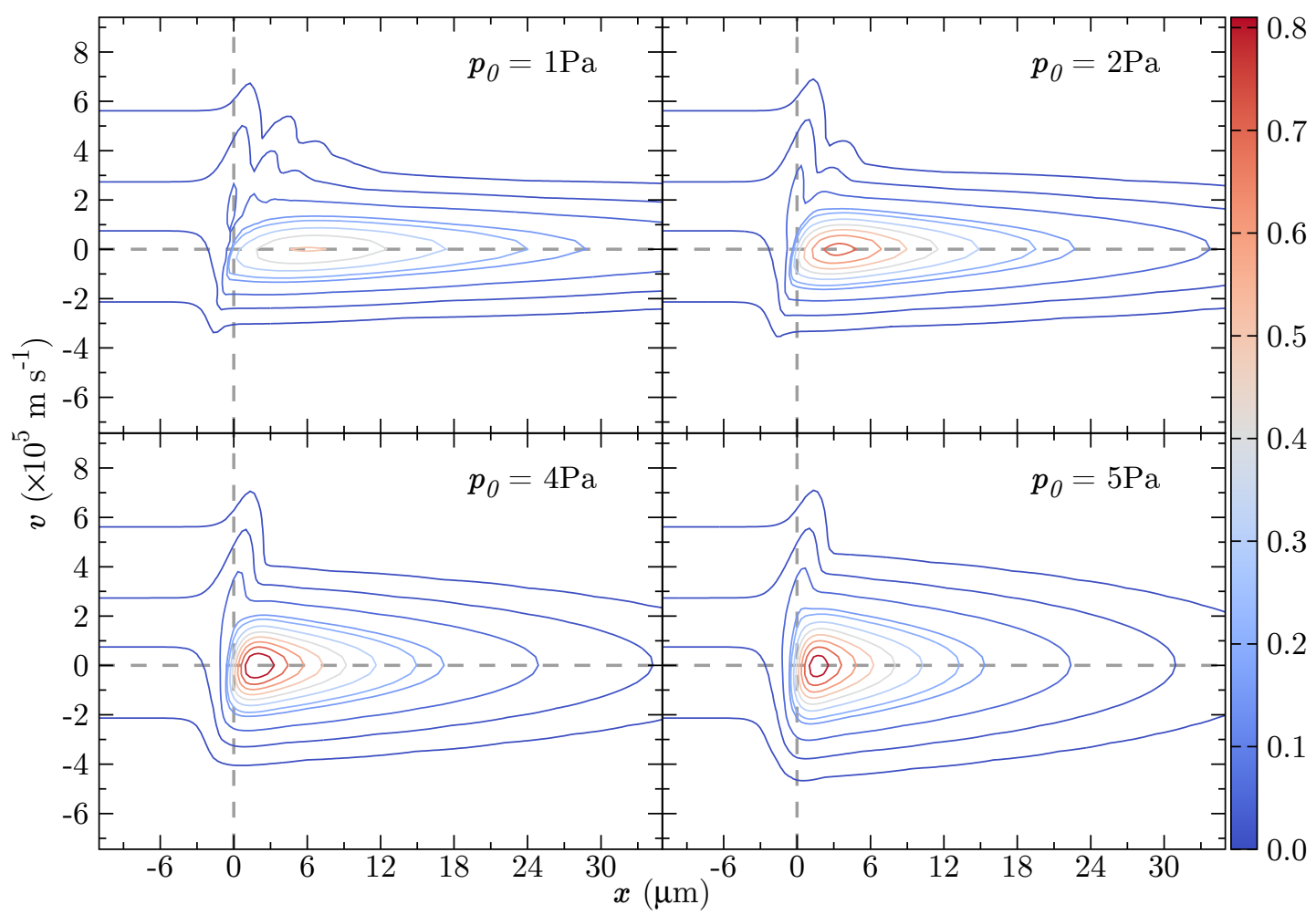

Figure 6.3: Contours of $f_{e} \times 10^{-4}$ at $T_{w}=2000 \mathrm{~K}$. The increment of pressure makes the peak of electrons higher and closer to the wall.

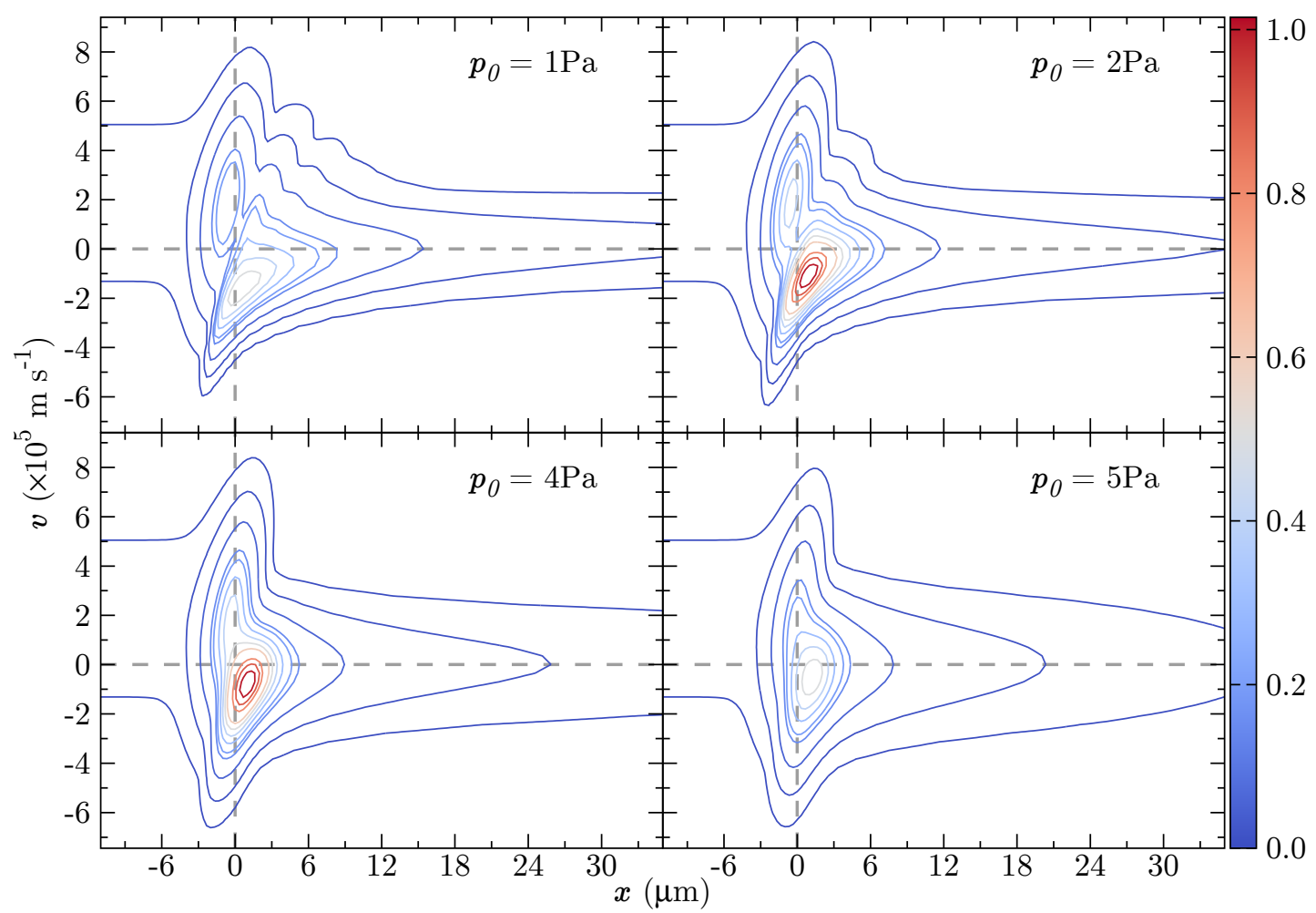

Figure 6.4: Contours of $f_{e} \times 10^{-2}$ at $T_{w}=2300 \mathrm{~K}$. A doubled peaked distribution can be clearly identified for low and intermediate pressures. 


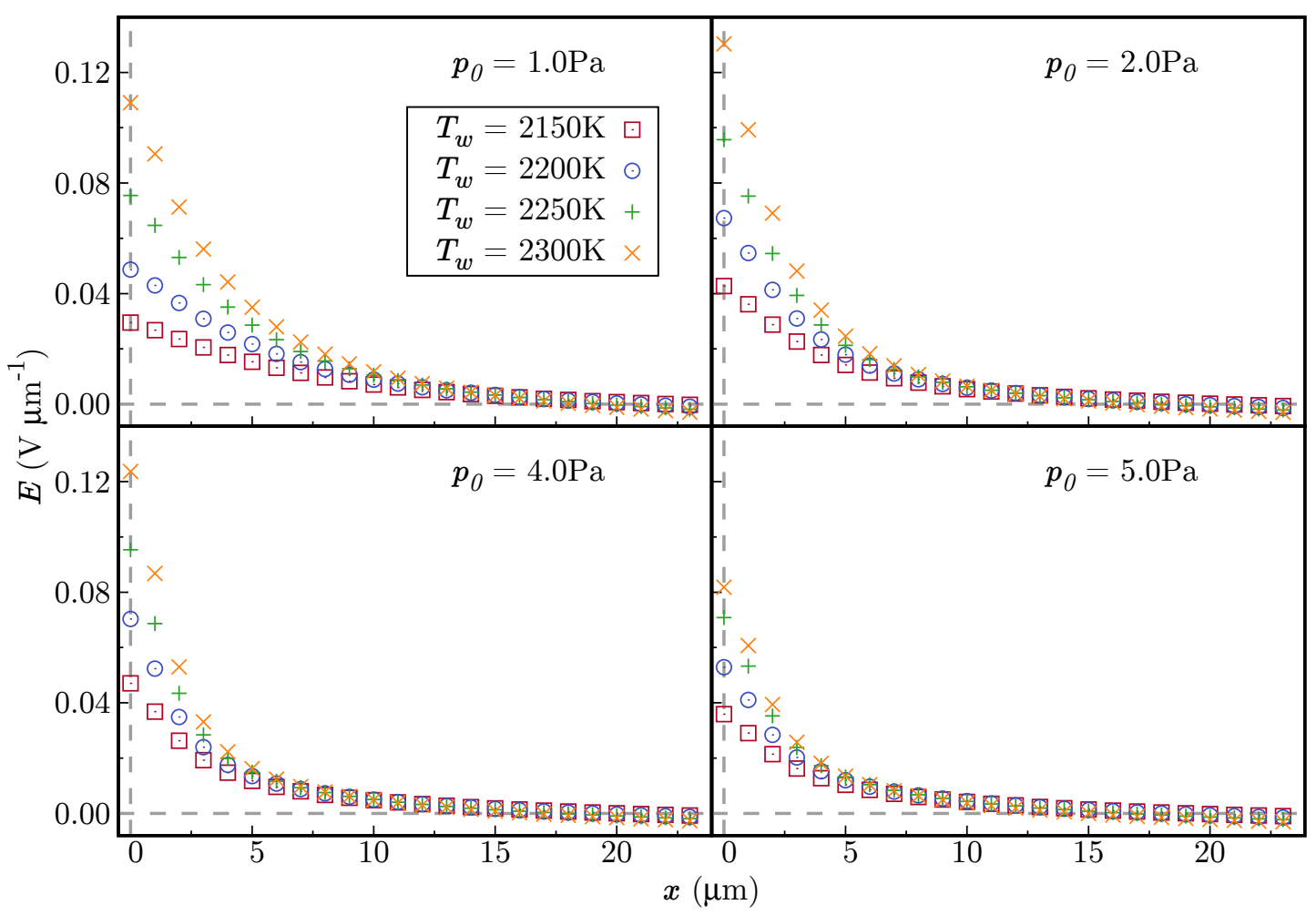

Figure 6.5: Electric field close to the wall for high temperatures. A change of sign appears at some position far from the wall.

a reduction of emitted current. As seen in figure 6.2, a huge difference between $p_{0}=4$ and $5 \mathrm{~Pa}$ appears, which is caused by the disappearing of the double peaked distribution function at $x=0$ for relatively high pressures. To better understand this variation, simulations with intermediate background pressures should be performed. The balance between the emitted electrons, self-consistent electric field and collisions with neutrals is responsible of the increment of $J_{T}$ at $x=0$, as presented in figure 6.1a. This interplay of very disparate dynamics can only be found by a self-consistent resolution of the discontinuous model. The PIM, once again, is proved to be an useful tool to this aim.

Another topic of major importance that can be studied with this model relies on the analysis of the self-consistent electric field far and close to the wall. The shape of $E$ is highly affected by two factors: the potential difference between the wall and the end of the numerical grid and the density of electrons at $x \geq 0$. For the cases presented in this section, the first parameter has been set to zero. Figures 6.3 and 6.4 show how the electron distribution function is affected by the neutral pressure and wall temperature, resulting in a modification of the electron particle density. Figure 6.5 pictures the self-consistent electric field for four high temperatures. As temperature increases, the density of emitted electrons also does, which enlarges the electric field, specially close to the discontinuity. The peak of $J_{T}$ found for intermediate pressures is caused by the intense electric field, resulting the two peaked distribution function found in figure 6.4. 


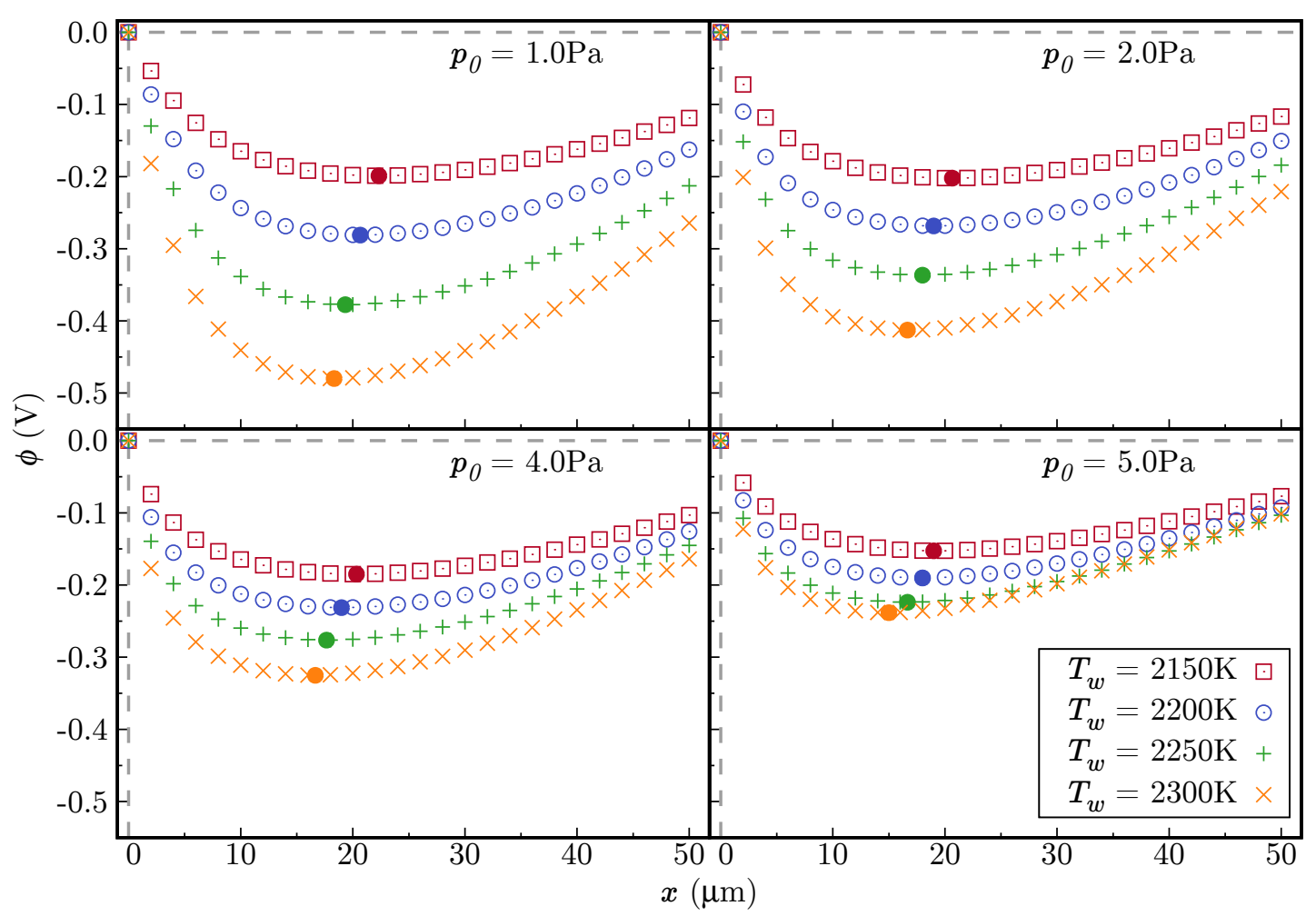

Figure 6.6: Electric potential $\phi$ for different temperatures and pressures studied. Potential well marked with a solid circle.

However, the electric field decays in a short distance, which indicates that the maximum amount of electrons is constrained very close to the wall, and only a small population of them escapes and reaches positions far from it. Due to the zero potential difference in the vacuum chamber, the electric field remains positive close to the wall, acting as a filter for low energy electrons. A change in the sign of $E$ is found in all cases. This indicates that the potential reaches a minimum point, shown in figure 6.6, where $\phi$ is pictured for the same cases of $T_{w}$ and $p_{0}$ as in figure 6.5. The point of minimum potential $\phi_{m}$ is marked as a solid circle.

The point of minimum potential tends to approach the wall by the increasing wall temperature and the background pressure, caused by the shifting of the electrons closer to the discontinuity. Consequently, as wall temperature increases, a deeper well is found [149], meaning that electrons require a higher energy to reach positions far from the wall. Nevertheless, pressure reduces this well, indicating that electrons must jump a lower potential barrier but they have more encounters with neutrals, which also reduce $J_{T}$. However, as pressure increases potential shapes become closer to each other, and specially for high temperatures they may become indistinguishable. In resume for an emitted electron to reach a certain position two barriers must be overpassed: the potential well and the collisional process with neutrals. Nevertheless, after an electron crosses the potential well, the $E$ makes it to increases its velocity and to overcome the collisions with the neutral gas. This phenomena is commonly found in experimental 
and theoretical models $[89,98,149,150]$ and it is usually referred to a potential well or virtual cathode. On the contrary, collisions affect all electrons entering the vacuum chamber. This means, the background gas always acts as an opposite effective force that thermalizes the emitted electrons, making a part of them return to the wall, limiting the emitted current. This barrier is usually neglected for low background pressures, but its real relevance depends on several factors, as the distance between the emissive wall and the vacuum chamber walls. It also depends on the background gas properties, as temperature, density and mass. In addition, ionization of the neutral background gas may appear [140, Section 4.1.1], which may also influence the simulation through variations in the self-consistent electric field.

To study the influence of the self-consistent electric field, the simulations are repeated by setting $E=0$ in all the vacuum chamber. Figure 6.7 depicts the current $J_{T}$ at $x=0$ with and without the self-consistent electric field. Close to the discontinuity, the positive electric field retains part of the emitted electrons, which causes the current increment for intermediate pressures. Without electric field, $J_{T}$ decreases an order of magnitude and behaves monotonic with pressure and the abrupt change at relatively high pressures disappears. As electron-neutral collisions become the relevant phenomena that leads the dynamics of the emitted electrons, the electric field influence decreases and both scenarios become more similar. In the same sense, figure 6.8 shows the comparison of emitted current with and without the electric field at $x=0.02 \mathrm{~mm}$. Different dynamics respect to the case at $x=0$ are found. Far from the wall, the absence of electric field increases the emitted current, meaning that without the potential well, more electrons reach positions farther inside the vacuum chamber as a result of suppressing one of the barriers that make electrons return to the wall. In resume, the self-consistent electric field generated by the emitted electrons retains them close to the wall, increasing $J_{T}$, but reducing the amount of electrons able to reach positions far from the metallic wall.

By the virtue of the previous discussion, we stress the relevance of the electric field, specially at low background pressures. This indicates that an accurate resolution of the Poisson's equation is required to properly describe the electrons in the vacuum chamber, as this field also influences the emitted current. In the model presented here, the electric potential is solved for the electrons in the vacuum chamber, but further improvements of the model should account also for the metal effect, as penetration of $E$ inside it, to obtain a continuous resolution of the electric potential. This task will be performed in future works as this new model opens the possibility for further, more accurate and deeper exploration. Moreover, simulations should be performed accounting for cylindrical geometry by two main reasons: the majority of emissive probes are shaped as wires, and the Poisson's equation is highly affected by the space geometry. However, this would require the development of new propagators to match the cylindrical geometry, which is a topic still under development. 


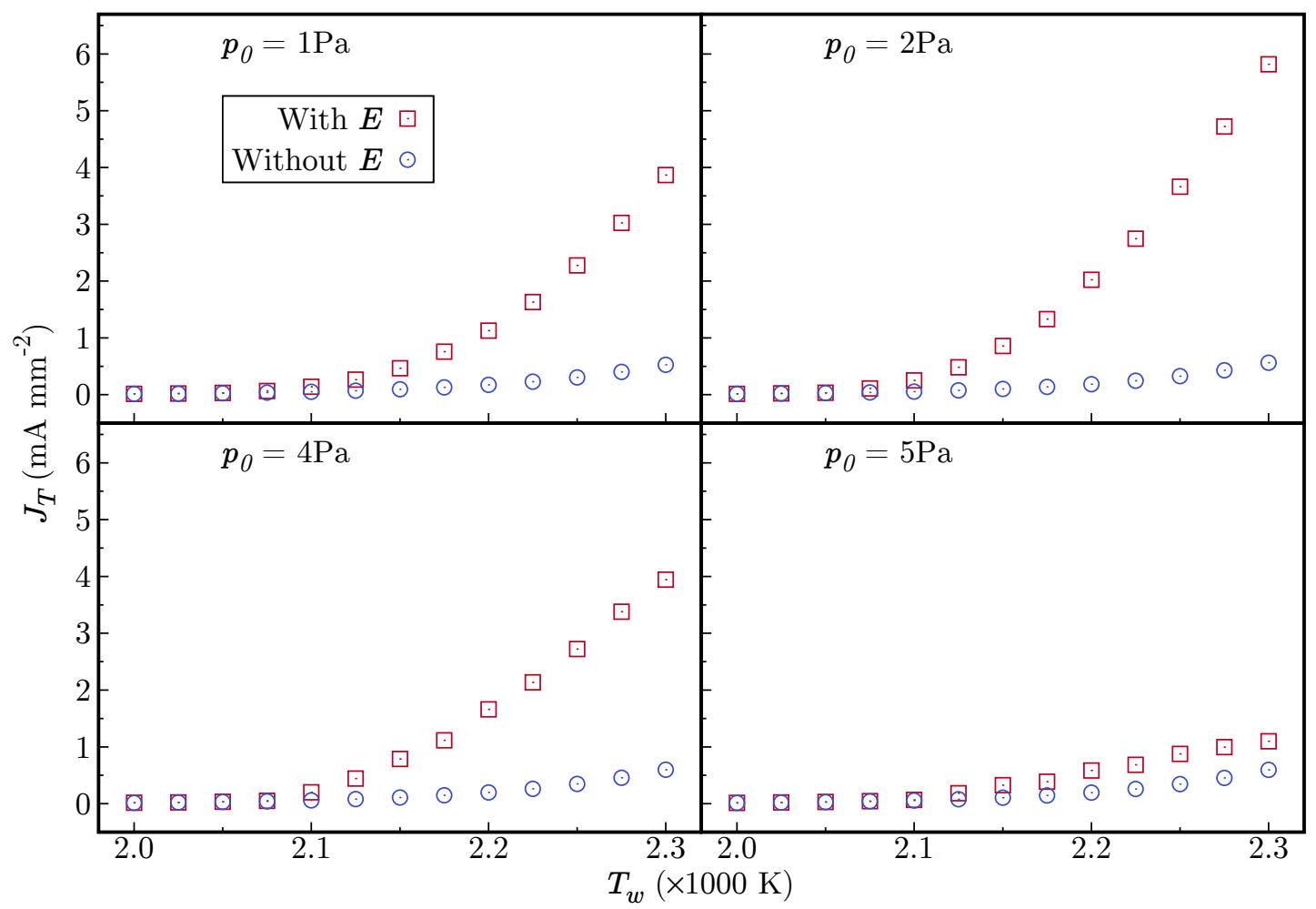

Figure 6.7: Comparison between the emitted current with and without the self-consistent electric field at $x=0 \mathrm{~mm}$.

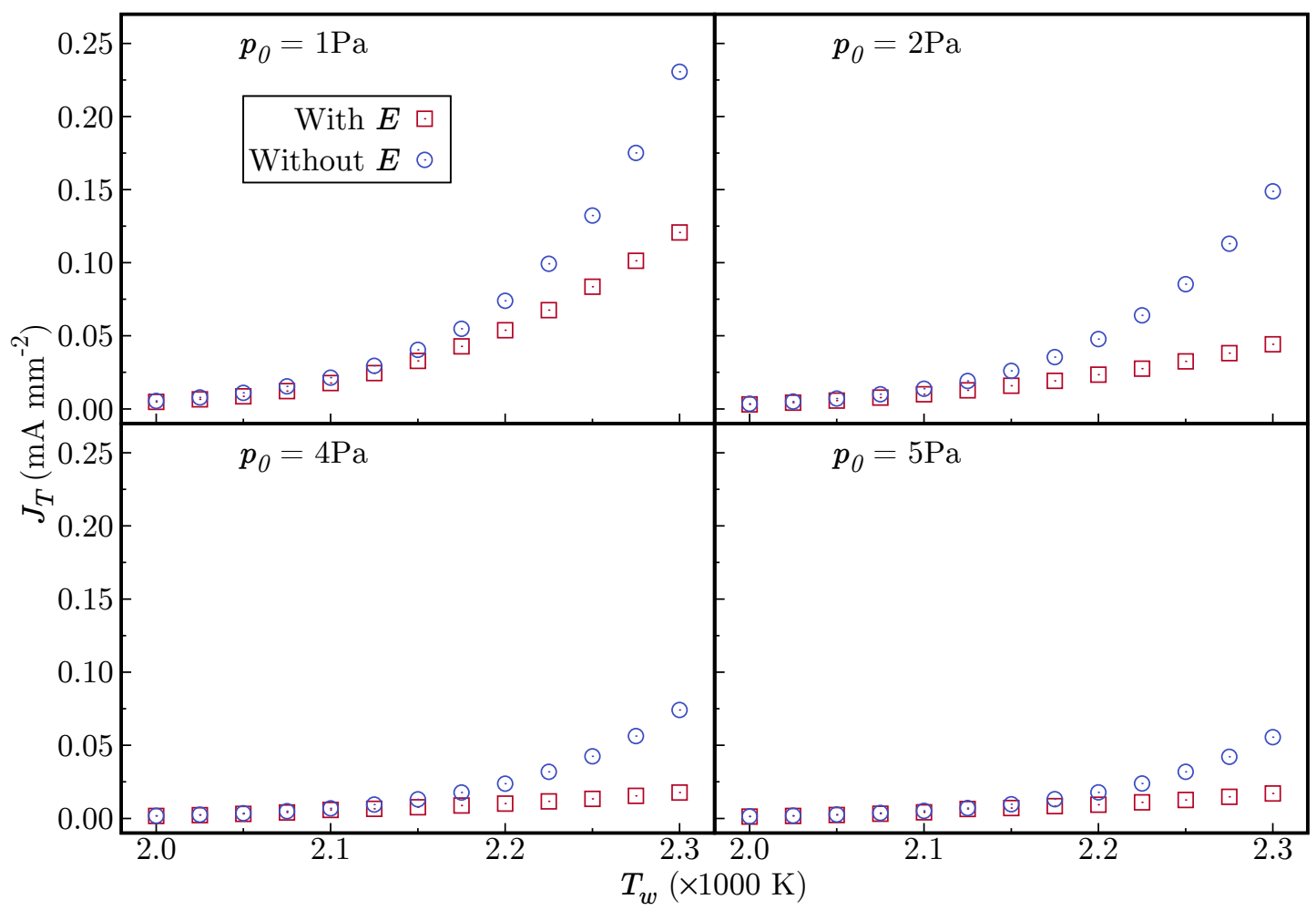

Figure 6.8: Comparison between the emitted current with and without the self-consistent electric field at $x=0.02 \mathrm{~mm}$. 


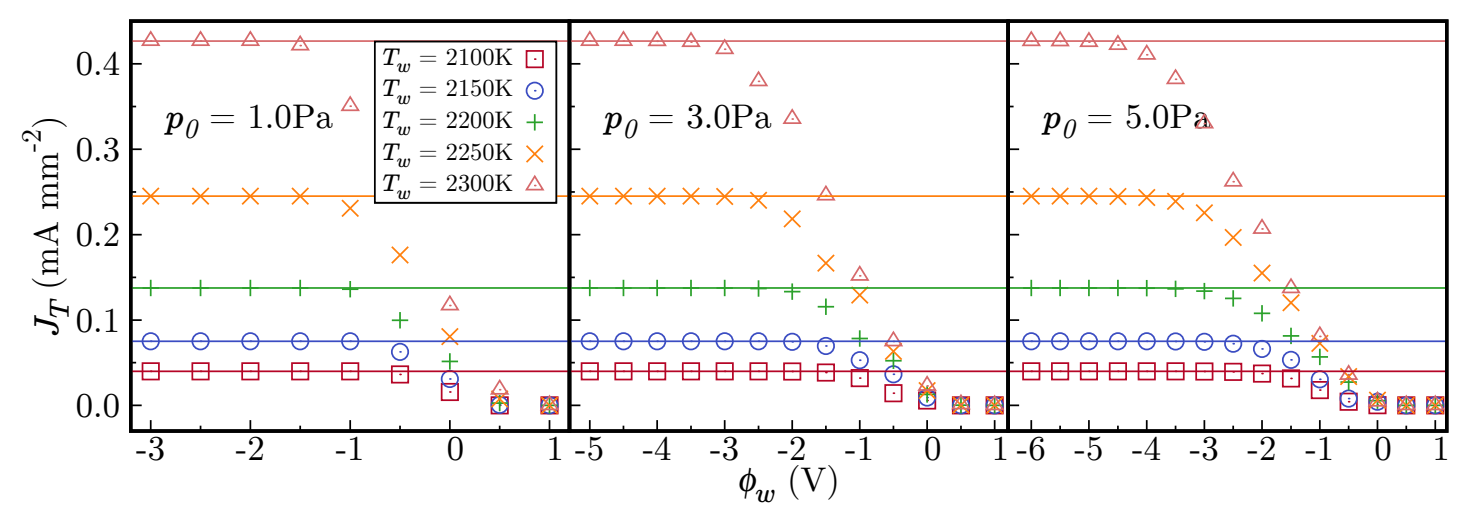

Figure 6.9: Emitted current $J_{T}$ at $x=0.02 \mathrm{~mm}$ for different wall potentials $\phi_{w}$. Solid lines represents the saturated current $J_{\text {sat }}$ obtained for the higher values of $\phi_{w}$

\subsubsection{Influence of the wall potential}

The shape of the self-consistent electric field computed with equation (6.2) is highly influenced by the potential difference between the wall and the vacuum chamber. In the cases presented above, this difference was set to zero, meaning that both boundaries were keep at the same potential. However, when emissive probes are employed as a measurement process, it is typical to bias the probe to a potential difference from the plasma or vacuum chamber walls to obtain a curve that relates the emitted current with the probe potential. To examine the influence of this potential jump, different values of the wall potential $\phi_{w}$ are used, covering a range of positive and negative values. It is expected that positive values reduce the emitted current, due to the more intense electric field close to the wall while negative values increase it, as the barrier imposed by the electric field reduces. In addition, a saturation scenario is expected to be reached when the electric field shape is such that no barrier appears for any emitted electron, regardless its energy, and the amount of returning electrons is minimum. The voltage difference required to reach the saturation current depends on the wall temperature $[149,157]$, but it is reasonable to assume that a dependency on the background pressure arises, since it imposes an additional barrier for the emitted electrons.

Figure 6.9 presents the emitted current far from the wall, $x=0.02 \mathrm{~mm}$, for five high wall temperatures as a function of the potential wall. It can be seen how a saturation current is found for certain values of $\phi_{w}$. This potential value, determined by $\phi_{s a t}$, varies with two parameters: the wall temperature and the background pressure. However, the value of saturation current $J_{\text {sat }}$, drawn as solid lines, is unaltered by the background pressure, since it is determined by the amount of electrons at the wall and they energy. Hence, the saturation of emitted electrons only depends of the properties on the wall, not on the dynamics inside the vacuum chamber, but the potential required to reach that current depend on the amount of neutrals, as well as the wall temperature. This evidences that an electron-neutral plasma is formed inside the vacuum chamber that collectively responds to changes in the wall potential. As stated above, electron-neutral interaction 


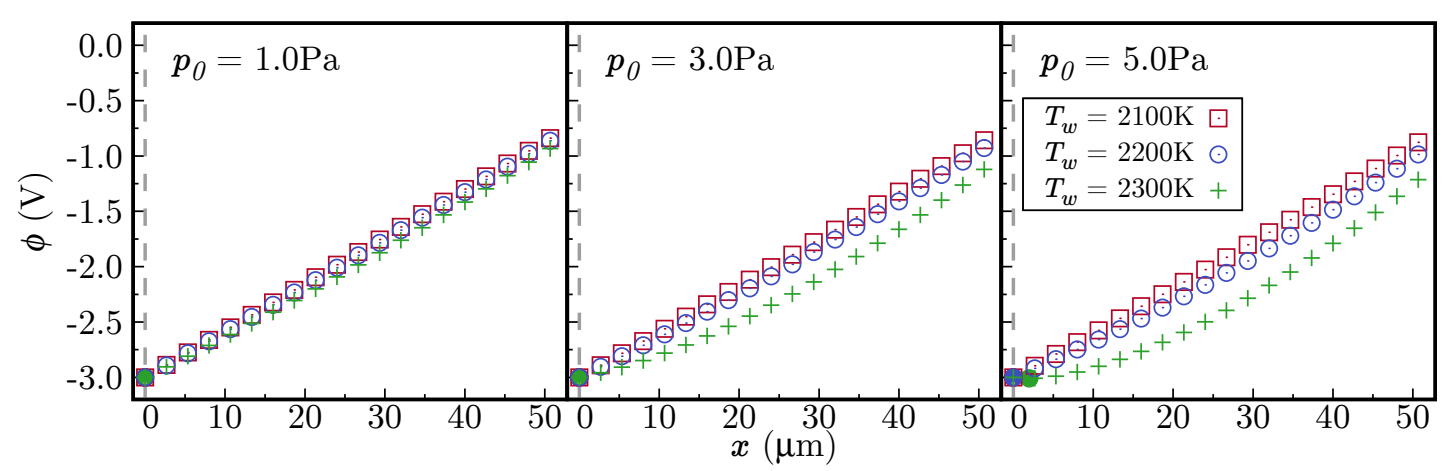

Figure 6.10: Electric potential for $\phi_{w}=-3 \mathrm{~V}$. Minimum potential point is marked as a solid circle for each case.

acts as an additional barrier to the one created by the self-consistent electric field that reduces the emitted electrons. However, when the potential difference between the wall and the vacuum chamber is large enough, electrons energy suffices to overcome this barrier. On the other hand, positive values of $\phi_{w}$ presented in figure 6.9, lead, as expected, to a reduction in the emitted current. This reduction is lower for small pressures, were collisions are not strong enough and emitted electrons have more energy to overcome the intense positively defined electric field.

Furthermore, it is expected that different laws to compute the properties in the wall, instead of Richardson-Dushman, produces a saturation current for a certain $\phi_{w}$. Even when the saturation current or the potential vary by different descriptions of the metallic wall, the saturation phenomena should remain. However, certain high emission conditions, as in a high density plasma discharge, or geometries could modify the plasma dynamics at the saturation zone.

At this point, it is relevant for our analysis to focus our attention on the potential shape for a situation when $\phi_{w}$ is different from zero. Figure 6.10 pictures the potential for $\phi_{w}=-3 \mathrm{~V}$. For this specific potential, emitted current at $p_{0}=1 \mathrm{~Pa}$ is saturated for all temperatures, as pictured in figure 6.9. However, as pressure increases, such a saturation is not found in all cases. For a situation where emitted current is saturated or close to saturation, potential becomes linear and the minimum of the potential appears at $x=0$. On the contrary, when the current is not completely saturated, a small well still appears, as seen for the case $T_{w}=2300 \mathrm{~K}$ and $p_{0}=5 \mathrm{~Pa}$. This well has the capability of retaining a small amount of electrons, meaning that part of them return to the wall. Moreover, a potential which shape is linear results in a constant and always negative electric field, which accelerates electrons in the whole vacuum chamber, making their energy increase and avoids the accumulation of electrons.

Hence, to evaluate the minimum $\phi_{w}$ once the emitted current is saturated, $\phi_{\text {sat }}$, does not seem to be an easy task since it depends on the wall temperature and the background pressure. Due to intermediate values have not been computed in this section, only an approximation $\phi_{\text {sat }}$ can be estimated, a more accurate $\phi_{\text {sat }}$ could rely between two derived values. Figure $6.11 \mathrm{a}$ pictures the minimum potential required to reach the 


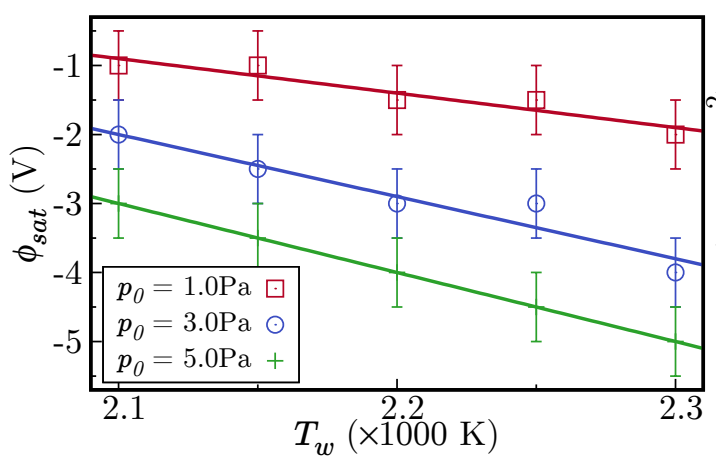

(a) Saturation potential

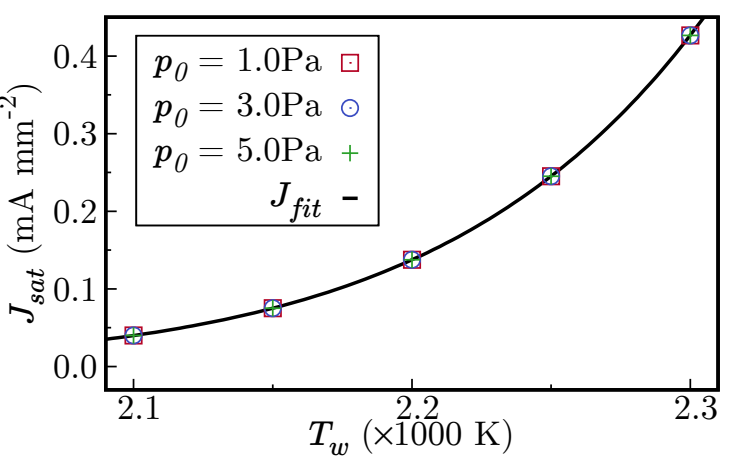

(b) Saturation current

Figure 6.11: Saturation potential (a) and current (b) as a function of wall temperature and pressure.

saturation current as a function of the wall temperature and the background pressure, with values limited by the wall potential chosen from figure 6.9. The behaviour of $\phi_{\text {sat }}$ with wall temperature is almost linear, and becomes more negative as the wall temperature and background pressure increase. The slope of $\phi_{s a t}$ with $T_{w}$ depends on the background pressure, as higher values of $p_{0}$ result on larger $\phi_{\text {sat }}$. In figure $6.11 \mathrm{~b}$ the current obtained at the saturation point is shown to check that this value is unperturbed by the background pressure. This proves that $J_{\text {sat }}$ only depends on the wall properties. Besides, $J_{\text {sat }}$ increases with the wall temperature following a similar law as equation (6.3), as described by the function

$$
J_{f i t}=C_{1} T_{w}^{2} \exp \left(-\frac{e C_{2}}{k T_{w}}\right)
$$

where $C_{1}$ and $C_{2}$ are parameters used to fit the function, taking the values

$$
C_{1}=7.551 \cdot 10^{5} \mathrm{~A} / \mathrm{m}^{2} \mathrm{~K}^{2} \text { and } C_{2}=4.549 \mathrm{eV}
$$

which agree with the values $C$ and $W_{f}$ from equation (6.3), respectively.

In conclusion, although the collisional scale for this problem is enlarged due to numerical constrains, it is clear that the neutral background gas influences the emitted current and that a strong coupling between the high energy emitted electrons, the selfconsistent electric field and the effect of electron-neutral collisions arise. Furthermore, the potential shape governs the electron population reaching a given position and acting as a filter due to the potential well generated between the wall and the vacuum chamber.

For a certain value of the wall potential, a saturation density current is found, which is only affected by the wall temperature, i.e., the amount of electrons available thanks to the initial condition in the wall. However, the voltage difference between the metallic wall and the vacuum chamber required to reach this scenario is influenced by the background neutral pressure, as well as the wall temperature. Moreover, the voltage shape also provides information about the saturation, finding a linear evolution, i.e., 


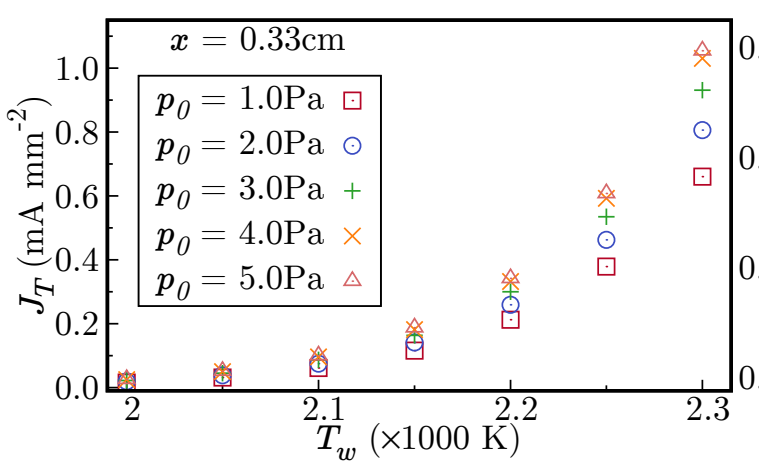

(a) Close to the wall

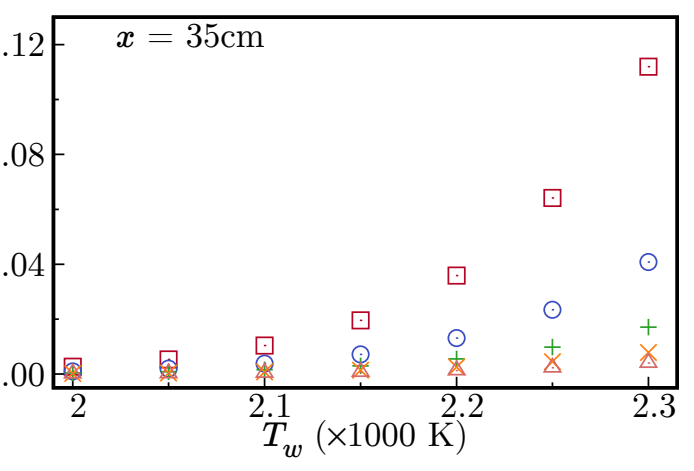

(b) Far from the wall

Figure 6.12: $\quad$ Emitted current as a function of temperature and background pressure for realistic collisional scales.

constant electric field, if the emitted current is saturated. If this saturation is not reached, the emerging potential well acts as a filter of low energy electrons. This well formation is influenced by the potential difference between the wall and the vacuum chamber, with the interplay of the wall temperature and neutral background pressure. Still, to specify the role of the background pressure in the emitted current, simulations are repeated with more realistic collisional scales in the next section.

\subsubsection{Realistic collisional scale without electric field}

In order to have a stable numerical scheme in previous results, the collisional scales must be artificially enlarged. This is caused by the strong electric field created as a result of the electron emission, which, in this simple calculation through equation (6.2) does not account for the metal and becomes sharp. Moreover, the planar geometry creates a much larger electric field than the one generated in a curvilinear space. However, to prove that the increment of background pressure effectively influences the electron emission into a vacuum chamber, simulations are performed here accounting for more realistic collisional scales but without the influence of the self-consistent electric field. For this aim we have $n_{c}=10^{20} \mathrm{~m}^{-3}$ and $\sigma_{c}=10^{-19} \mathrm{~m}^{2}$, values close to the characteristic $e-0$ collision frequencies.

The emitted current close to the wall, and a position far from it, is shown in figure 6.12. Due to the large characteristic length, the position far from the wall in which the emitted current is analysed is $35 \mathrm{~cm}$, much closer to the position of a detector or the vacuum chamber enclosure in a real experimental setup. It can be seen that close to the wall, figure $6.12 \mathrm{a}$, emitted current increases with the background pressure $p_{0}$ meaning that electrons are retained due to the increasing electron-neutral collision. Moreover, this behaviour is linear, contrary to the one found for figure 6.1a, where $J_{T}$ reached a maximum for an intermediate value of pressure. It can be assumed that the non-monotonic behaviour results from the interplay of elastic collisions and the self-consistent electric field. 
With respects to positions far from the wall, figure $6.12 \mathrm{~b}$, the emitted current decreases for increasing $p_{0}$, consistently with the results found in the previous section. It is clear that, even when the self-consistent electric field is missing in these simulations, collisions with the background gas influence the emission of electrons into a vacuum chamber. In spite of the results found here, more accurate models and simulations are still required. This model established that electron-neutral collisions play a important role in the dynamics of emitted electrons. The description of plasma-wall interaction as a discontinuous media opens a wide range of possibilities to describe this complex system, accounting for physical phenomena in both, the metal and the vacuum chamber. However it proper description requires the development of numerical tools, propagators, and the capability to solve big demanding computational resources problems, which are out of our reach at this point.

\subsection{Collecting wall}

When the plasma is bounded by a wall capable of collecting charged particles, its dynamics describes a very complex system, related to the rapid charge separation obtained and the modification of the plasma dynamics caused by the collection of particles. In this section, we present a kinetic model to describe ions and electron dynamics of a weakly ionized plasma close to a collecting wall, with no previous assumptions about the density or electric potential shapes. The kinetic model in the $1 \mathrm{D} 1 \mathrm{~V}$ planar phase space employed is mathematically expressed as

$$
\begin{gathered}
\frac{\partial f_{\alpha}}{\partial t}+v \frac{\partial f_{\alpha}}{\partial x}+\frac{q_{\alpha} E}{m_{\alpha}} \frac{\partial f_{\alpha}}{\partial v}=\left(\frac{\partial f_{\alpha}}{\partial t}\right)_{\alpha 0} \\
\frac{\partial^{2} \phi}{\partial x^{2}}=-\frac{e}{\epsilon_{0}}\left(n_{i}-n_{e}\right) \\
\phi(0)=\phi_{w} \\
\left.\frac{\partial \phi}{\partial x}\right|_{x \rightarrow \infty}=0
\end{gathered}
$$

where $\left(\partial f_{\alpha} / \partial v\right)_{\alpha 0}$ is a Dougherty collision term for charged particles interactions with neutrals and $\phi_{w}$ is the potential of the collecting wall, situated at $x=0$ and $\alpha=e, i$. To solve this system, a slabbing method is employed instead of directly using the 1D1V propagator from section 2.2.2. This approach allows a better representation of the process in the $v$ direction and reduces the number of points required to represent the $x$ direction. This slabbing method is appropriate because the dynamics in the $x$ direction is dominated by the convective flux of particles and not the spurious diffusive process. This slabbing method consists on numerically evaluate the flux of particles $v \partial f_{\alpha} / \partial x$ and replacing it in (6.7) by a non-homogeneous term that represent the particle balance through the slab. With this approach, it is possible to avoid some issues presented in the description of $1 \mathrm{D} 1 \mathrm{~V}$ cases, in section 2.2.2, as boundary conditions and the large amount of points required in the $x$ direction. Similar approach was employed in [43], 


\begin{tabular}{lclll}
\hline & Mass $(\mathrm{kg})$ & Velocity $(\mathrm{m} / \mathrm{s})$ & Temperature $(\mathrm{eV})$ & Density $\left(\mathrm{m}^{-3}\right)$ \\
\hline e & $9.10938356 \times 10^{-31}$ & $\sqrt{\frac{k T_{i}}{m_{i}}}$ & 1 & $r_{i} \frac{n_{T}}{2}$ \\
$\mathrm{i}$ & $72819.6 \times m_{e}$ & $\sqrt{\frac{k T_{i}}{m_{i}}}$ & 0.1 & $n_{e}$ \\
0 & $m_{e}+m_{i}$ & 0 & 0.01 & $n_{T}\left(1-r_{i}\right)$ \\
\hline
\end{tabular}

Table 6.1: Argon plasma to analyse the collecting wall by a one-dimensional slabbing method.

where a source term was used to introduce a heat flux to compute transport coefficients of a two-dimensional plasma in the velocity space, based on the pioneer work by J. P. Matte and J. Virmont [158].

To analyse the behaviour in the different slabs, the flux term in the $x$ direction is evaluated as

$$
-v \frac{\partial f_{\alpha}}{\partial x} \simeq s_{f l u x}(x, v, t)=\left\{\begin{array}{ll}
-v \frac{f_{\alpha}(x+\Delta x, v, t)-f_{\alpha}(x, v, t)}{\Delta x} & \text { if } v<0 \\
-v \frac{f_{\alpha}(x, v, t)-f_{\alpha}(x-\Delta x, v, t)}{\Delta x} & \text { if } v>0
\end{array},\right.
$$

coming from the physical sense of the divergence of $\mathbf{v} f$ per unit of volume. Here, $\Delta x$ represents the separation between two consecutive slabs. This is consistent with the explanation provided in 2.2.2 about the possible boundary conditions, where the flux of $f$ through a surface must be positive, i.e., $\mathbf{n} \cdot \mathbf{v}>0$. Applying this approximation to equation (6.7), the resulting one-dimensional problem for each slab becomes

$$
\frac{\partial f_{\alpha}}{\partial t}+\frac{q_{\alpha} E}{m_{\alpha}} \frac{\partial f_{\alpha}}{\partial v}=\left(\frac{\partial f_{\alpha}}{\partial v}\right)_{\alpha 0}+s_{f l u x}
$$

As initial condition for the plasma we assume a quasi-neutral argon weakly ionized plasma with the parameters from table 6.1 carried out into drift Maxwellian distributions. This initial condition ensures that a large number of particles reach the wall at $x=0$, but there will always be an amount of returning ones to the quasineutral plasma, due to collisional effects or the action of the self-consistent electric field. To modelise the charge-neutral collisions, constant cross-sections are used as $\sigma^{i 0}=10^{-18} \mathrm{~m}^{2}$ and $\sigma^{e 0}=10^{-20} \mathrm{~m}^{2}$ with a total density of particles $n_{T}=10^{19} \mathrm{~m}^{-3}$ and the ionization ratio $r_{i}=10^{-6}$. Charge-neutral collisions vary their intensity through the thermal velocity, as presented in chapter 5. Charge-charge collisions are neglected due to their weak influence in species dynamics for this ionization ratio, as presented in figure 5.5. Due to the upwind slabbing, a small $\Delta x$ must be employed, which constrains the evolution to a reduced time step $\tau=2 \cdot 10^{-5}$ to ensure that the upwind discretisation of the flux in the $x$ direction is stable from a numerical point of view. This slightly perturbs the mesh-free basis of the PIM, but it is a requirement to ensure that the transversal flux is well represented. A total of 200 slabs is utilized, covering the space from $x=0$ up to $x=40 \mathrm{~cm}$. In the first slab, a total absorbing boundary condition is imposed, meaning that $f_{\alpha}(0, v>0, t)=0$. The distribution function for $f_{\alpha}(0, v \leq 0, t)=0$ is accounted as a one-dimensional semi-open problem in the 


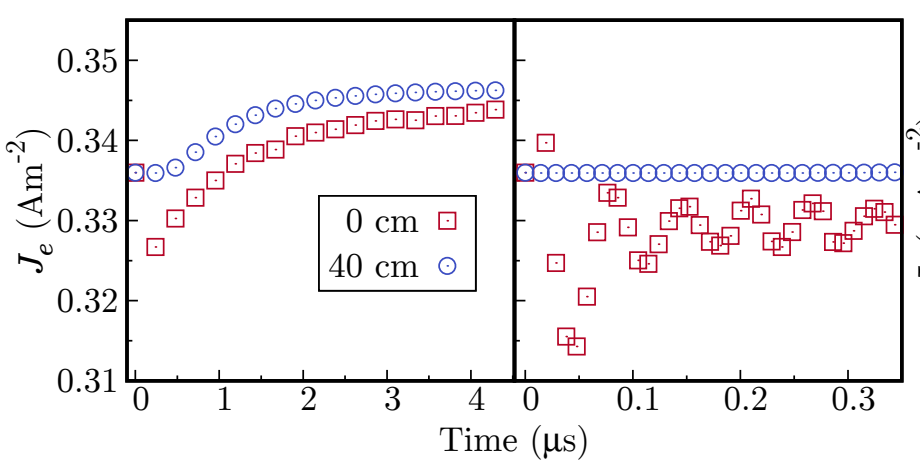

(a) Electrons

(b) Initial perturbation

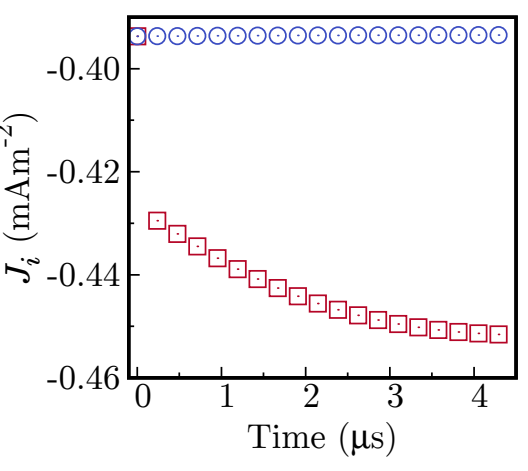

(c) Ions

Figure 6.13: Density current for electrons and ions at $x=0$ and $x=40 \mathrm{~cm}$.

\begin{tabular}{ccc}
\hline$\omega_{p e}(x=0)$ & $\omega_{p e}(x \rightarrow \infty)$ & $\omega_{p e}$ Numerical \\
\hline 115.633 & 126.166 & 106.461 \\
\hline
\end{tabular}

Table 6.2: Electron plasma frequencies close to the collecting wall. All values are expressed in $\mathrm{rad} / \mu \mathrm{s}^{-1}$.

region $(-\infty, 0]$ with the boundary condition $f_{\alpha}(0,0, t)=0$. At $x=40 \mathrm{~cm}$, the distribution function for negative velocities is kept constant to sustain the plasma while the distribution at positive velocities is perturbed by the downstream flux and collisional processes.

The density current, $J_{\alpha}(x, t)=\left[q_{\alpha}\right]$ is plotted at $x=0$ and $x=40 \mathrm{~cm}$ in figure 6.13 . The wall acts as an initial perturbation that disrupts the quasi-neutral plasma. In figure $6.13 \mathrm{~b}$ it can be seen how electrons move faster, creating an oscillatory situation that is dumped after a time of $1 \mu \mathrm{s}$ approximately. After the initial perturbation, plasma adapts itself to the new situation, but each species make it at its own rate. The wall effect is quickly felt by the electrons, due to their high energy and low mass, and it can be seen how the point far from the wall is influenced as positive velocity particles decrease, leading to a more positive electric current. Electrons behave in an oscillatory way to adapt to the sudden perturbation of the wall. These oscillations, of unique frequency, are related to the plasma frequency $\omega_{p e}$, approximated for cold plasmas as

$$
\omega_{p e}=\sqrt{\frac{4 \pi n_{e} e^{2}}{m_{e}}}=2 \pi f_{p e} \approx 2 \pi 8980 \sqrt{n_{e}} \mathrm{rad} / \mathrm{s},
$$

where $n_{e}$ is the electron density, with all magnitudes in cgs units. For this expression, ion mass has been assumed to be infinite. Table 6.2 presents the plasma frequency for three situations. First, two cases from equation (6.11) using the electron density at $x=0$ and far from the wall at a time of $t \simeq 0.15 \mu \mathrm{s}$, which correspond to the approximated values of $4.2 \cdot 10^{6}$ and $5 \cdot 10^{6} \mathrm{~cm}^{-3}$ respectively. Then, the result obtained extracting the mean frequency from the cycles in figure $6.13 \mathrm{~b}$ is presented. It can be seen how all frequencies are in the same order of magnitude of $100 \mathrm{rad} / \mathrm{s}$, but the 


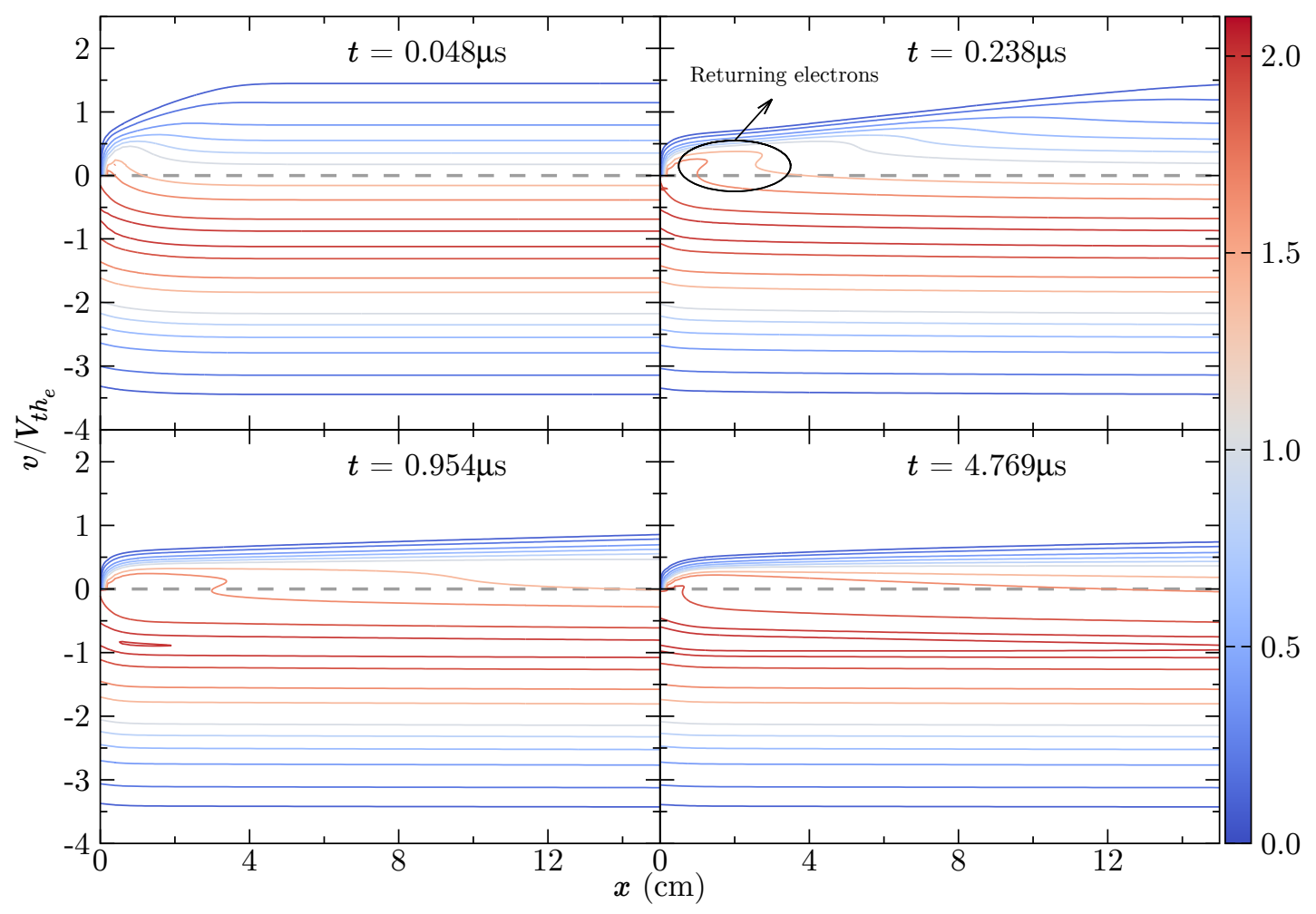

Figure 6.14: Contours of $f_{e} \times 10^{-7}$ in arbitrary units at different time frames. The relaxation of the returning electrons is clearly appreciated for $t<1 \mu \mathrm{s}$

numerical value is slightly lower than the analytical one for $x=0$. Hence, it is assumable to say that the electrons are evolving in the simulations close to its own frequency. Moreover, this result arises naturally from the self-consistent resolution of the model, without imposing any constrain. The self-consistent resolution of the electric field is crucial to obtain this result, since small variations in it are the main reason of the electron oscillatory state. So, our simulations produce a evolution that resembles physical properties of the plasma. Moreover, an evolution of ions is appreciated if figure $6.13 \mathrm{c}$, meaning that this heavy species reacts to the perturbation produced by the wall, but in a larger time scale than electrons.

Figure 6.14 pictures the contours of $f_{e}$ at four frame times. For short times, the effect of the wall only reaches positions close to it, but as time increases, the influence in the distribution function extends, and an important part of electrons is repelled by the metallic wall. The wall highly influences the positive velocity part of the distribution function, which perturbs the quasi-neutral plasma. Some works, as [21,89], include a non-homogeneous term to maintain the quasi-neutral plasma from a certain position. In the actual model, this task is achieved only by the boundary conditions at $x=40 \mathrm{~cm}$. This means that all the domain can be affected by the presence of a metallic wall. The use of an external source-sink of particles to maintain the plasma could perturb the system evolution [159], forcing an specific electric potential structure. Nevertheless, the boundary conditions at $40 \mathrm{~cm}$ are a difficult task to establish. To properly describe 


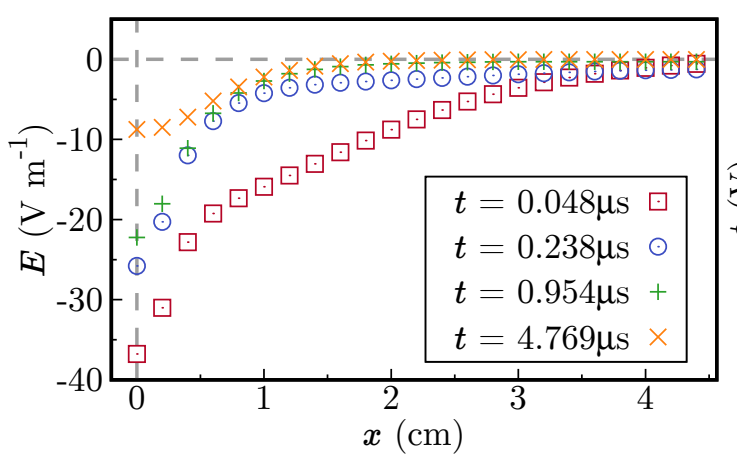

(a) Electric field

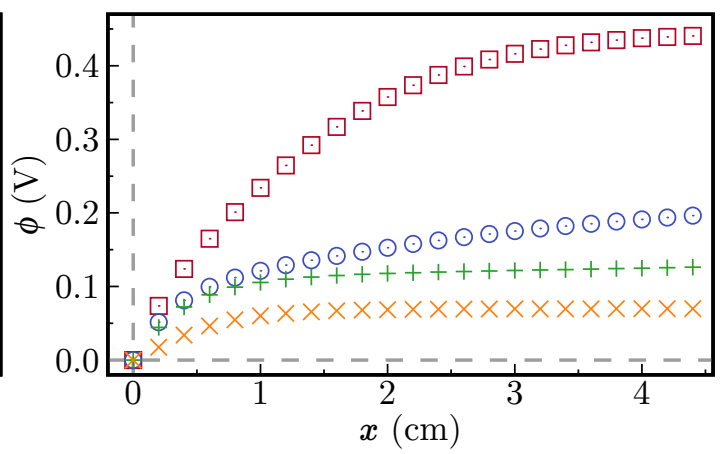

(b) Electric potential

Figure 6.15: (a) Electric field and (b) potential close to a collecting wall. Monotonic evolution is found for the potential, but interesting variations close to the wall arise for the electric field.

the quasi-neutral plasma, enough space in the $x$ direction should be provided to ensure that the system recovers a quasi-steady state without external terms. However, the computational time required to achieve that number of slabs is not available right now.

The boundary condition at $x=0$ means that no particles are introduced into the numerical domain. However, it can be seen how a population of electrons still holds for $v>0$. This is caused by the amount of electrons that return due to effect of the self-consistent electric field and the collision with neutrals. To analyse the first cause, the electric potential and field are pictured in figure 6.15. It can be seen how the fast evolution of electrons generates a large electric field due to the charge separation evolving to eliminate it by accelerating electrons positively and ions negatively. The electric field reduces its intensity as time increases and the potential difference between the wall and the end of the numerical domain is reduced drastically, as the plasma self-consistently adapts to the boundary condition. This creates some of the returning trajectories identified in figure 6.14.

\subsubsection{Effect of ions dynamics}

Although ions evolve in a slower time scale than electrons, its dynamics is relevant to the system dynamics [21], specially for relatively large evolution times. Figure 6.13 shows the fast evolution for electrons, with an oscillatory state for short times, and the quasi-steady state for times in the order of $\mu \mathrm{s}$. Ions take longer time, greater than $\mu \mathrm{s}$, to adapt to the new situation. In the results presented here, it is not clear if ions have reached a quasi-steady situation, as an oscillatory response in a much larger time scale is expected. The influence of the ions dynamics for short and long time scales is studied in figure 6.16, where the electron density current $J_{e}$ at $x=0$ with and without accounting for the ion evolution are compared. For small evolving times, figure 6.16a, both evolutions agree. However, for longer times, quasi-steady electron state differs completely. This is mainly caused because ions evolution is only relevant 


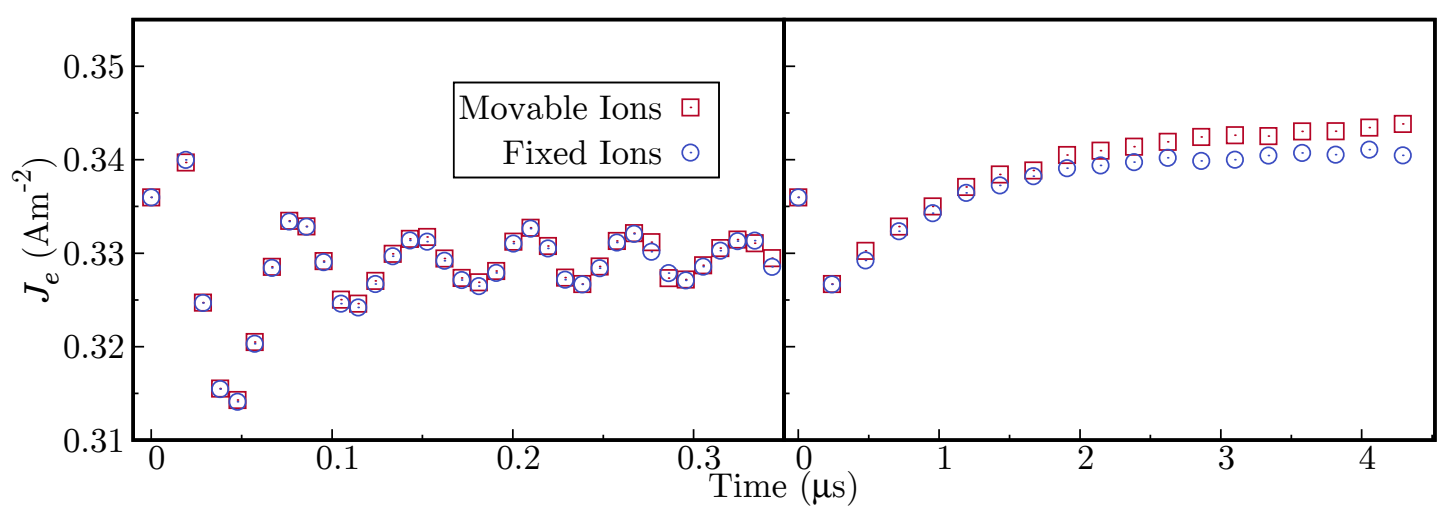

(a) Electrons

(b) Initial perturbation

Figure 6.16: Comparison between electron evolution with and without advancing the ions in time. Similar evolutions for short times appears, while quasi-steady situation changes drastically.

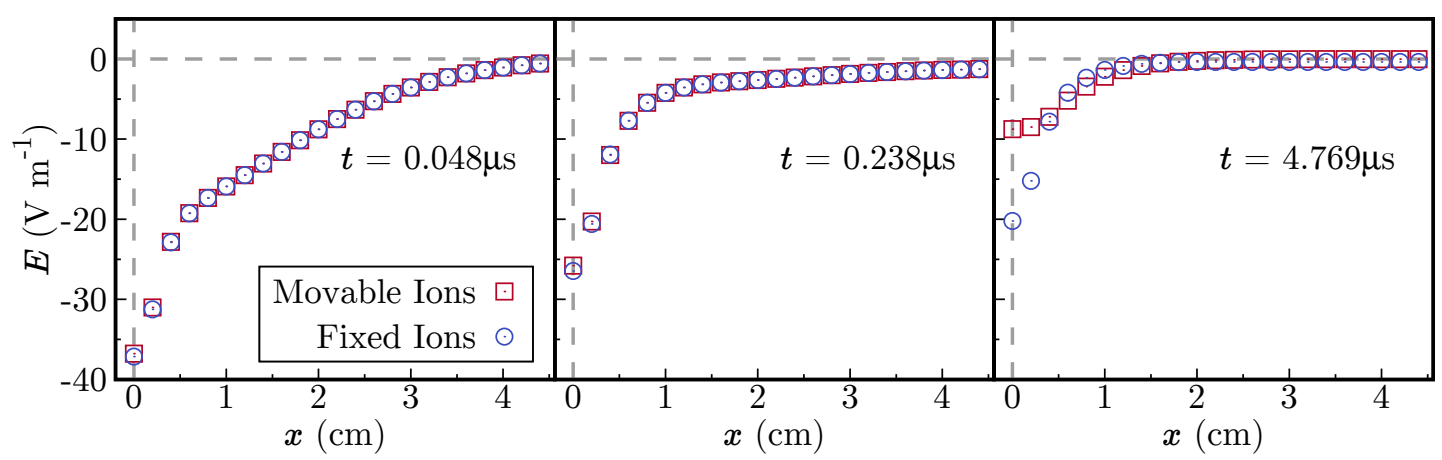

Figure 6.17: Comparison between self-consistent electric field with and without advancing the ions in time. Ion evolution reduce the electric field close to the wall as they are being collected.

for large times. Nevertheless, the time intervals analysed are in the order of $\mu \mathrm{s}$, while in weakly ionized plasmas measurements and evolutions may take place in scales close to the ms. This means that to study WIP with a coupled Poisson's equation the presence and dynamics of ions is relevant for scales larger than the $\mu$ s. Neglecting the evolution of this species and the influence that it has in the Poisson's equation can result in a completely different dynamics and steady state.

Because of the only coupling between ions and electrons dynamics comes from the Poisson's equation, the choice of leaving the ions fixed and infinitely massive creates a significant difference in the self-consistent electric field, specially for long times as shown in Figure 6.17. It can be seen how the electric field found at the end of the simulation differs close to the wall if ions evolution is accounted for. When ions are evolved, an ion collection at $x=0$ appears, its density is reduced, resulting in a small charge separation. However, if ions are fixed at their initial condition, i.e., ion density is uniform and static, a electric field of high intensity is found close to the collecting wall as a result of the enlarged charge separation. It is expected that this difference increases for larger evolution times. 


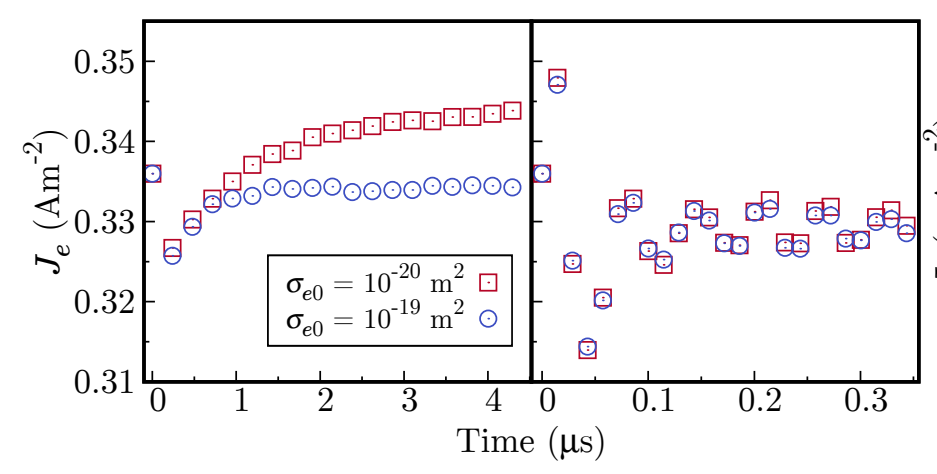

(a) Electrons

(b) Initial perturbation

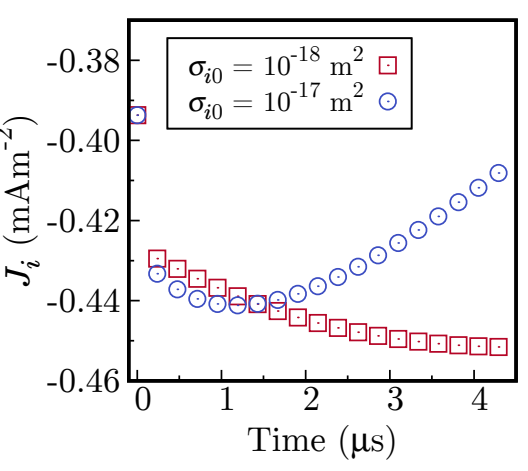

(c) Ions

Figure 6.18: Density current for electrons and ions at different values of cross-section.

\subsubsection{Influence of charge-neutral collisions}

Another interesting topic is to study the effect of the charge-neutral interaction in the plasma close to a collecting wall. To this aim, simulations with different values of $\sigma^{\alpha 0}$ are presented for the cross-sections ten times the ones employed above.

The density current at $x=0$ for short times $6.18 \mathrm{~b}$ the electrons evolve similarly. However, as time increases, differences appears and quasi-steady states of both species slightly differ. Nevertheless, these differences are small, $\sim 0.01 \mathrm{~A} / \mathrm{m}^{2}$ and do not significantly modify the electrons state. A faster electron relaxation is found, meaning that collision frequency could influence the response time when the system is perturbed.

On the other hand. ions, figure $6.18 \mathrm{c}$, do not reach a quasi-steady situation as collisions with neutrals results in a fast response of this heavy species. This indicates that ion relaxation times are highly influenced by collisional effects and are much larger than the total time studied here. In view of this, it is assumable that in previous simulations ions have not reached a stationary state but their characteristic relaxation time is much larger than the simulation time. Additional simulations should be performed to really identify the ions dynamics, as its influence at longer times is not clarified with these results.

\subsection{Conclusions}

The very involved and interesting plasma wall interaction phenomena has been modelled and numerically solved by two distinguishable models. This problem is of huge importance in the characterization of plasmas and its dynamics in bounded domains. Many disparate and odd processes determine the dynamics of these systems, as the distribution and energy of emitted electrons, the self-consistent electric field and the effects appearing in the vacuum chamber in which plasma exists. Kinetic descriptions of these phenomena are an arduous task, but they provide relevant information, helpful 
to understand the processes involved and how they influence the interaction. In our models, no assumption about the distribution function or the potential field is assumed, since they are obtained from a pure self-consistent kinetic resolution.

A discontinuous plasma-wall model to describe the electrons emitted and collected back by a high temperature metallic planar wall has been presented, for the first time, in this chapter. Influence of the background pressure created by the neutrals gas inside the vacuum chamber has been analysed, finding that electron-neutral elastic interaction plays a very important role in the description of the emitted particles, specially for relatively high background pressures. Moreover, the self-consistent electric field has been found to be of mayor importance to properly describe the electron dynamics in the vacuum chamber. The potential difference between the metallic wall and the vacuum chamber is therefore important as a saturation process appears for certain values of $\phi_{w}$. This saturation means that no barrier is imposed by the electric field and all particles are emitted to the metal. A similar relation to the Richardson-Dushman law is found when the saturation current $J_{\text {sat }}$ is analysed as a function of the temperature. The saturation current only depends on the wall properties, but the potential required to reach this scenario $\phi_{\text {sat }}$ depends on both, wall properties, mainly its temperature, and the background pressure. Although these results are a first step in the description of a troublesome scenario and simulations must be improved to account for more realistic scales and geometries, some dynamics are similar to those found in the laboratory, as the decrement of emitted electrons due to the increment in neutral density or the saturation of the emitted current for a certain wall potential.

In addition, the description of ions and electrons in the presence of a collecting wall has been carried out. Even when mass of this species is huge compared with electron one, its dynamics close to a collecting wall modifies the electric field, as ions are being collected by the wall and its density is being reduced, resulting in a lower charge-separation. At the same time, collisions between charges and neutrals are also important at kinetic levels. This is due to electrons and ions dynamics being highly influenced by the interaction with neutrals, specially in the case of ions where non-steady situations are clearly identified. Larger simulation times are required to properly describe the ions dynamics, as well as the possible variation in time of wall conditions, as electric potential or emission of particles, to extend the applicability of this model.

Once more, the numerical stability and properties of the Propagator Integral Method have been satisfactory tested. Physical results are obtained as a consequence of the self-consistent resolution of involved models, including the effect of the electric field or charge-neutral collisions. The future works to translate these models into cylindrical and spherical geometries to match the typical probes shapes will be carried out. More microscopic process should be included to analyse its influence, as well as a more accurate description of the self-consistent electric field in the case of the discontinuous wall emission model. 



\section{CHAPTER 7}

\section{CHARACTERISATION OF A PLASMA THRUSTER PLUME}

Through out this memory, a wide class of scenarios, related to those found in experiments, have been solved by means of the Propagator Integral Method (PIM). In this chapter, a final important application of deep interest, in relation to an actual research area of our group, is presented. Here the PIM is applied to modelise the plasma plume generated by an electric thruster. By plume we refer here to the exhaust jet produced by the plasma thruster. These plasmas are characterised by a mesothermal flow in the axial direction, meaning that ion characteristic velocity is larger than the thermal one but lower than electron thermal velocity. However, an expansion and relaxation of the plasma jet occurs in the three-dimensional space, which is usually composed by the radial and axial directions respect to the thruster axis. The importance of the plume characterization resides in knowing the dispersion suffered by charged particles to avoid interferences with other parts of the spaceship. Moreover, to have some control over the thruster operation, it is important to analyse the main system parameters that control the thrust obtained.

The basic procedure to generate thrust in plasma propulsion systems consists on the acceleration of ions by means of intense electromagnetic fields [8,160,161]. To accomplish this, a neutral gas is ionized inside a chamber and ions are then extracted and accelerated while electrons are kept inside the chamber to contribute to the ionization process. With these systems, a high specific impulse can be achieved, and thrust usually lies in the order of $\sim \mathrm{mN}$. These characteristics provide a propulsive system suitable for long missions where fuel payload is kept as low as possible. Orbit corrections, flight formation, interplanetary travels, or end-of-life disposal of small satellites are missions for which plasma propulsion is a promising durable long-lasting research field. 
The characterization of the plasma generated by these thrusters is not an easy task, either from experimental or theoretical points of view. Fluid models and particle-incell codes are usually employed for the modelisation of the plasma generated by the thruster, which can leave behind microscopic phenomena that are of huge importance to properly describe the ion energy distribution functions in the plume. Alongside this, numerical resolution is specially difficult, since it is imperative to deal with nonlinear problems and proper evaluation of transport phenomena. An extensive review of numerical methods employed to describe electric propulsion thrusters can be found in [162] and references therein.

In this chapter, a kinetic model to describe the ions in a plasma plume is presented. The main collisional processes that generate the ion distributions found in experimental observations are analysed. In particular, we pay attention to the plasma plume generated by ALPHIE (Alternative Low Power Hybrid Ion Engine) [100,127] of figure 7.1, a low power ion thruster in current development by the UPM PlasmaLab. This thruster consists on a set of magnetic crowns that generate a field inside the chamber to trap electrons coming from the active cathode neutraliser that ionize the neutral gas introduced through the inlet. Two grids are situated at the thruster exhaust, a cathode and an control grid. The main propose of these two grids is to retain electrons inside the discharge chamber, to contribute with the ionization process, and to control the exhaust of ions that provides the thrust. All the system is DC powered, and therefore this thruster is categorized as an electrostatic one.

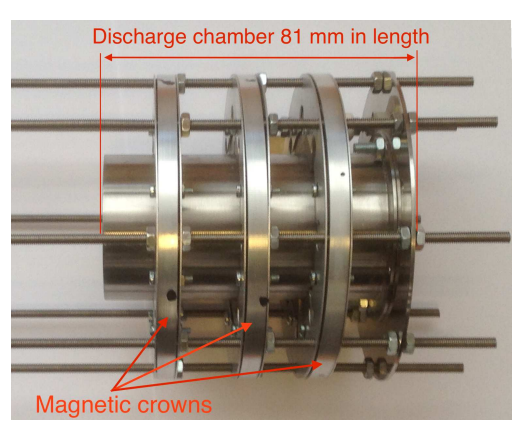

(a) ALPHIE plasma thruster

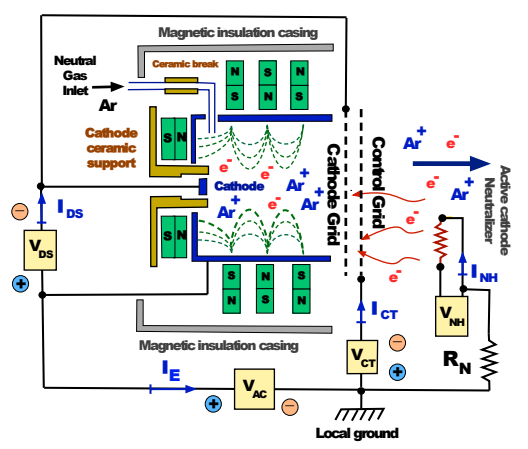

(b) Electrical scheme

Figure 7.1: (a) The ALPHIE plasma thruster chamber and magnetic crowns and (b) its electrical scheme. All credit correspond to the experimental team of the UPM PlasmaLab.

However, the model presented in this chapter is not applicable only to describe the ALPHIE's plume but it is intended to be of general use for other thrusters with similar operation modes and physical properties. The aim of this chapter is to elucidate the basic phenomena, at microscopic scale, observed in the laboratory, and to obtain a first kinetic description of the plasma plume. 


\subsection{Experimental motivation}

As previous said, one of the main objectives of this chapter is to describe the physical processes that generate the ion energy distribution function found in experiments. Hence, it is useful to firstly present some of the results obtained by the experimental team of the UPM PlasmaLab by means of a Retarding Potential Analyser (RPA) [118, 127]. Main results are summarised in figure 7.2. In these measurements, a double peaked ion energy distribution function (IEDF), figure 7.2a, is found as being the superposition of 2 distributions: one corresponding to the accelerated and the other corresponding to the thermal population of ions. In this figure, $Q$ is the mass flow introduced to the inlet and $p_{A}$ is the vacuum chamber pressure in which the measurement process takes place. As the control parameter $V_{A C}$ increases, the thermal population of ions become more energized, while the thermal peak remains more or less constant, figure 7.2b. A relaxation process takes places along the plume, figure 7.2c, as the accelerated population of ions vanishes and only the thermal one remains. Meanwhile fluid models could properly account for the macroscopic plume evolution, a distribution function suitable to compare with experimental results can only be achieved by scription.

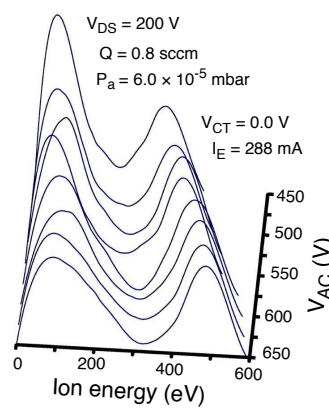

(a) Effect of $V_{A C}$

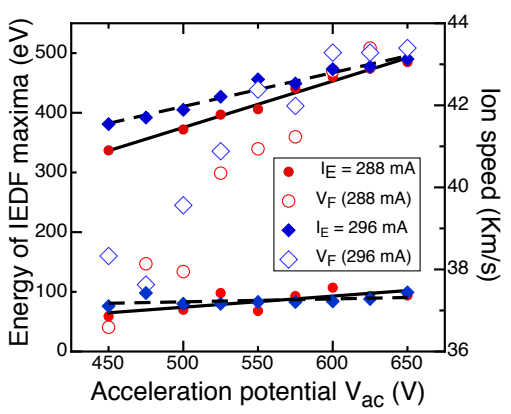

(b) Energy maxima

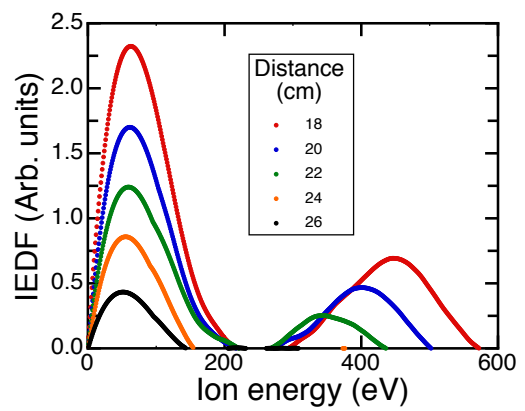

(c) Relaxation

Figure 7.2: $\quad$ Measurements of the ion energy distribution function by means of a RPA performed in the low temperature plasma laboratory of the UPM PlasmaLab. (a) Effect of increasing $V_{A C}$. (b) Energy of the accelerated and thermal maxima as a function of $V_{A C}$. (c) Relaxation of the ion beam as axial coordinate $z$ increases.

\subsection{Ion kinetic description in the thruster plume}

In this section, a kinetic model to describe the evolution of ions at different positions in the axial direction of the ALPHIE's plume is studied. The aim of this basic model is twofold: firstly, to study the microscopic processes that produce the double peaked distribution function found in the experiments and, secondly, to obtain a kinetic description of the thruster plume capable of reproducing different realistic plasma scenarios.

The Propagator Integral Method (PIM) is used to account for elastic collisions and 


\begin{tabular}{lccc}
\hline & $n_{\gamma}\left(\times 10^{18} \mathrm{~m}^{-3}\right)$ & $K_{\gamma}(\mathrm{eV})$ & $T_{\gamma}(\mathrm{eV})$ \\
\hline Neutrals (for all $z)$ & $1-r_{i}$ & $m_{0}\left(10 V_{t h}^{0}\right)^{2} / 2$ & 0.0431 \\
Ions (at $z=0, v>0)$ & $r_{i} / 2.0$ & $K_{0}$ & 0.0431 \\
\hline
\end{tabular}

Table 7.1: Initial conditions (density, kinetic energy and temperature) for neutrals and ions. Subscript $\gamma$ references ions and neutrals.

non-homogeneous terms (as charge-exchange) and obtain a time evolving 1D1V ion distribution function. The slabbing method introduced in section 6.2 is employed to discretise the plume parallel direction. Ions interact with a static and uniform background of neutrals by means of elastic collisions and charge-exchange, presented in sections 4.1.4 and 4.3, respectively. Even when these two exchanges produce similar macroscopic evolutions, the high energy ion flux coming from the thruster creates significant variations in the distribution function that influences the system evolution. Electrons and self-consistent electric field do not influence the dynamics of the ions. It is reasonable to assume that the high energy of ions produced by the thruster, in the order of $\sim 100 \mathrm{eV}$, are insignificantly affected by the interaction with the light species or by the low electric field, in the order of $\sim 1 \mathrm{~V} / \mathrm{m}$. However, their influence should be analysed in depth for more involved descriptions. Nevertheless, the approach discussed here provides a first kinetic description of ions and analyses the microscopic phenomena that affect their distribution in the velocity space.

To describe the ions in the thruster plume, the motion equation

$$
\frac{\partial f_{i}}{\partial t}=\left(\frac{\partial f_{i}}{\partial t}\right)_{i 0}+s_{f l u x}+s_{e x}^{i}
$$

is solved for each slab in a $1 \mathrm{D}$ open velocity space, where $s_{f l u x}$ is the flux along $z$, the parallel direction, described by equation $(6.9), s_{c-e}$ is the charge-exchange term between ions and neutrals presented in section $4.3,\left(\partial f_{i} / \partial t\right)_{i 0}$ is the elastic Dougherty collisional term from section 4.1.4 and $v$ is the velocity associated with direction $z$. The properties for ions and neutrals are in table 7.1, where $K_{0}=m_{i} u_{i}^{2} / 2$ is the kinetic energy of the ions at the first slab, $u_{i}$ its associated velocity, usually in the range of $40-50 \mathrm{~km} / \mathrm{s} ; r_{i}$ is the density ratio between ions and neutrals and $V_{t h}^{0}=\sqrt{k T_{0} / m_{0}}$ is the thermal velocity of neutrals. Drift-Maxwellian distributions functions are assumed as initial conditions. It is clear that the flux of ions accelerated by the thruster is represented here by a distribution function of high kinetic energy, i.e., high mean velocity, and low temperature, resulting in a Dirac delta like distribution function. However, this shape is an assumption as a more accurate ion distribution should be obtained from other models or experimental measurements.

In the different simulations performed in this chapter, ions starts only as a single $\delta$ shaped population in the first slab, which is fixed through the simulation because ion extraction is assumed as uniform and stationary. As stated in section 2.2.2, boundary conditions can only be imposed in a constrain range of $v$. Equally to section 6.2, some 
parts of the boundaries have a distribution function different from zero. In this problem, the first and last slabs are used as boundary conditions to confine the system evolution. The first plasma slab impose the drifted distribution function for ions for $v \geq 0$ and solves the ion distribution function in the domain $v<0$ assuming a total absorbing boundary condition at $v=0$ to ensure a continuous $f$. At the last slab, a total collective wall is assumed to represent a probe positioned far from the thruster exhaust. This is, $f_{i}$ is zero for $v<0$ and that the distribution function is solved for $v>0$ with null $f_{i}$ at $v=0$. To numerically solve the problem, all variables are dimensionalised by its reference values, represented by the superscript 0 , which are

$$
\begin{gathered}
n^{0}=10^{18} \mathrm{~m}^{-3} ; m^{0}=m_{i}=6.627 \cdot 10^{-26} \mathrm{~kg} ; q^{0}=e ; \\
\sigma^{0}=10^{-19} \mathrm{~m}^{2} ; v^{0}=V_{t h}^{i} ; T^{0}=T_{i} ; \\
\mu^{0}=\sigma^{0} v^{0} ; \nu^{0}=n^{0} \mu^{0} ; t^{0}=\frac{1}{\nu^{0}} ; E^{0}=1 \mathrm{~V} / \mathrm{m} ; L^{0}=v^{0} t^{0} .
\end{gathered}
$$

A number of 20 slabs, separated a distance of $2 \cdot 10^{-3}$ dimensionless units is employed, with a time step $\tau=2 \cdot 10^{-6}$. A total of 5001 points are employed in each slab to discretise the velocity space, covering a distance from $\sim-100$ up to $\sim 260$ non-dimensional units, to allow the representation of high and low ion velocities. All results are presented in a quasi-steady situation reached after 1000 iterations, which corresponds to a time of $\sim 60 \mu \mathrm{s}$. For these simulations, ions and neutrals have the same mass as their difference, the electron mass, is minimum when the reference scale is the ionic one.

\subsubsection{Influence of charge-exchange and elastic collisions}

Now, the influence of the collision processes between ions and neutrals is studied. For all simulations presented in this section, $K_{0}=500 \mathrm{eV}$ and $r_{i}=10^{-5}$. Firstly, the effect of charge-exchange is analysed. To do so, different cross-section for this process are tested in the simulations and the distribution of ions through the slabs is analysed. Three cases varying the charge-exchange cross-section value are presented: no chargeexchange $\left(\sigma^{e x}=0\right)$, a constant cross-section $\left(\sigma^{e x}=5 \cdot 10^{-19} \mathrm{~m}^{2}\right)$, and the case with an energy dependent cross-section as the one employed by M. Lampe et al. [121], taking the form

$$
\sigma^{e x}=5.53 \cdot 10^{-19}(1-0.0543 \ln \epsilon) \mathrm{m}^{2}
$$

where $\epsilon$ is the kinetic energy, in $\mathrm{eV}$ corresponding to the relative velocity $\left|v-v^{\prime}\right|$. A cross-section of $\sigma^{i 0}=10^{-18} \mathrm{~m}^{2}$ is employed to describe elastic collisions between ions and neutrals.

Figure 7.3 pictures the ion distribution function $f_{i}$ for the different cases of chargeexchange cross-section at three positions in the axial direction. The first relevant result extracted from this figure is that no thermal peak appears without charge-exchange. This means that this process is the main mechanism that produces a population of thermalised ions, i.e., ions at the energy level of neutrals. Moreover, part of the high 


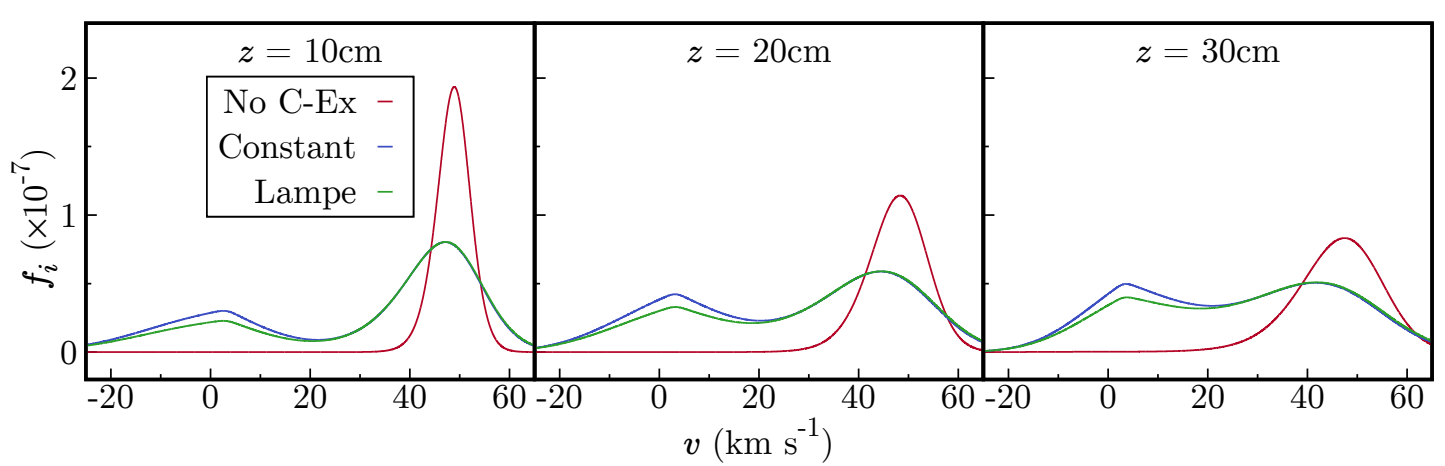

Figure 7.3: Influence of the charge-exchange in the ion distribution function at three scenarios: without charge-exchange (No C-Ex), with a constant cross-section (Constant), and with the cross section from equation (7.3). Thermal peak only appears when this collision is accounted for.

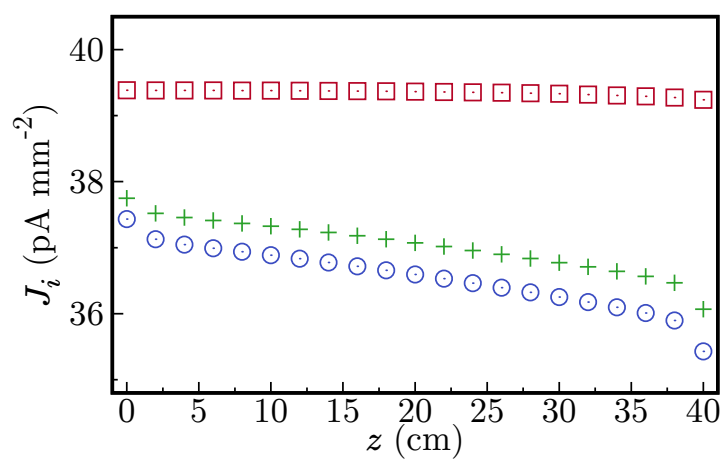

(a) Ion current density

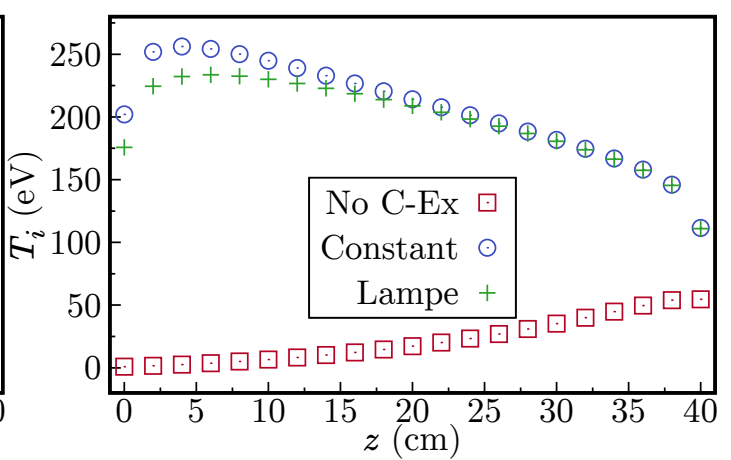

(b) Ion temperature

Figure 7.4: Influence of the charge-exchange in the current density and temperature for the ions. With charge exchange, the current density is highly reduced while a temperature orders of magnitude higher than the initial one is reached.

energy flow is transferred to the neutral background, resulting in a macroscopic reduction of the ion energy. When no charge-exchange is accounted for, $J_{i}=\left[q_{i}\right]$ is only slightly affected by the boundary condition at $z=40 \mathrm{~cm}$, but remains almost constant through out the plume, figure 7.4. For this case, temperature only increases as a result of the elastic collision between ions and neutrals. On the other hand, when chargeexchange is accounted for, similar behaviours are found, regardless if the cross section depends on the relative velocity. As $z$ increases, the thermal peak grows, meaning that a determined length scale is required for this peak to appear and stabilize. Also, the appearance of the thermal peak highly increase the ion temperature, resulting in a more intense elastic collisions as $\mu^{i 0}$, the collision parameter related with the ion-neutral elastic exchange, is proportional to the thermal velocity. It is important to stress that this temperature is computed using the distribution function in all the velocity space, as usual in this dissertation. In all cases, it can be seen how ions distribution function suffers a relaxation in a certain length, meaning that ions of a large variety of velocities arise, which agrees with the experimental results presented in figure 7.2a. 


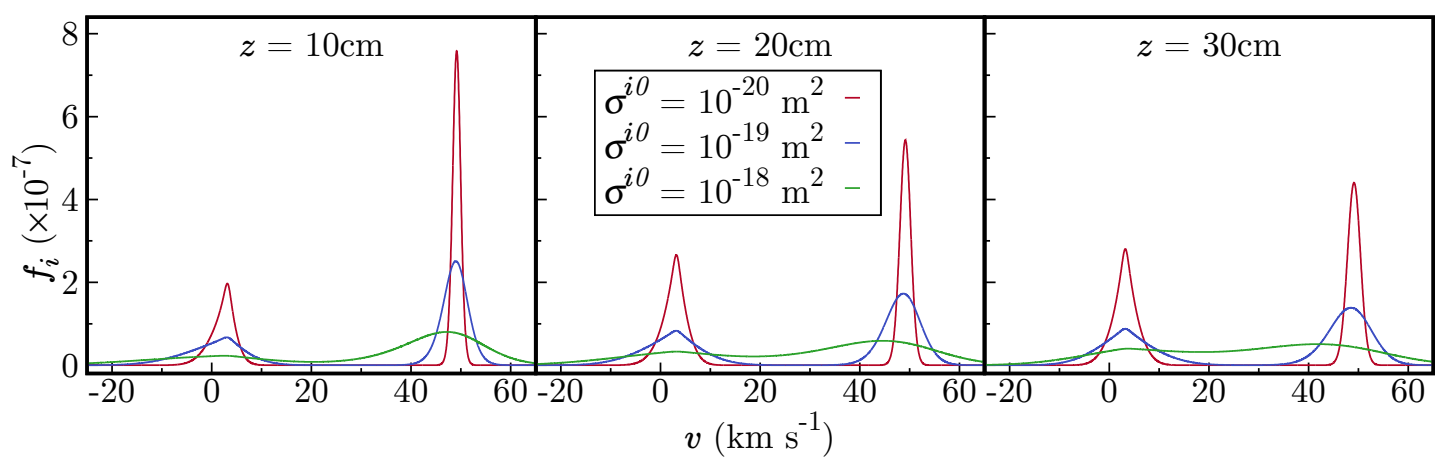

Figure 7.5: Influence of the elastic collision in the ion distribution function at three different positions.

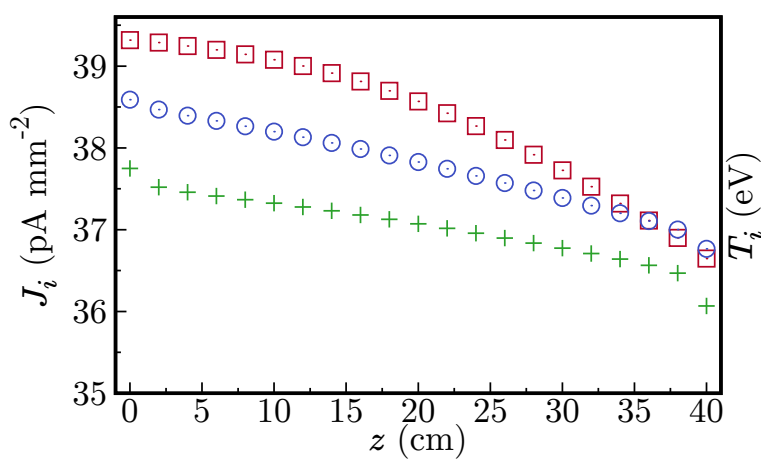

(a) Ion current density

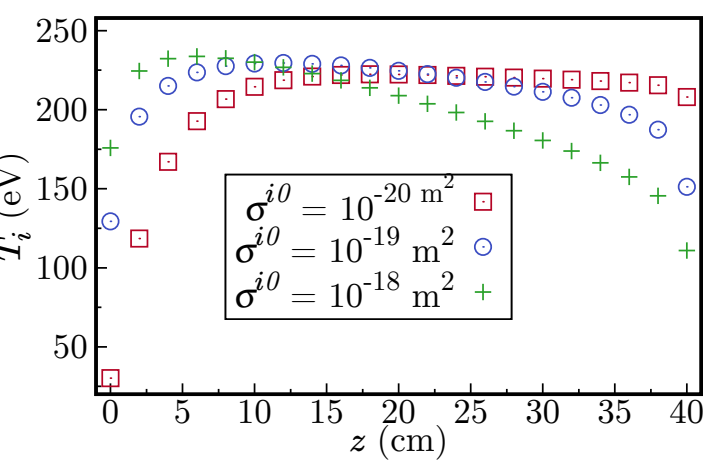

(b) Ion temperature

Figure 7.6: Influence of the elastic collision in the current density and temperature for the ions. Elastic collisions reduce the ion density current and slightly increase temperature.

The influence of ion-neutral elastic exchange is also studied for some constant values $\sigma^{i 0}=10^{-18}, 10^{-19}$ and $10^{-20} \mathrm{~m}^{2}$. Figure 7.5 shows how $f_{i}$ varies with different collision cross-sections along the plume axial direction. For low values of $\sigma^{i 0}$, two differentiated maxima are found, as the distribution function between them vanishes, meaning that no relevant population of ions exists on that velocity range. As collision frequency increases, the spread of ions also develops, increasing the probability to find an ion at velocities between the two main peaks. However, the situation found in the experimental measurements where ions fill a wide range of velocities between both peaks is only recovered in the case of $\sigma^{i 0}=10^{-18} \mathrm{~m}^{-2}$. It is clear that the kinetic model studied here reproduce the relaxation of the plasma plume obtained in the experiments if the cross-section for collisional processes have the appropriate values.

With respect to the current density and temperature for the ions in the plume, figure 7.6 shows its evolution for different $\sigma^{i 0}$. Elastic collisions between ions and neutrals increase the ion temperature and reduce the current density for all $z$. However, it can be seen how small cross sections values result in an uniform evolution of the temperature for all positions. The effect of the absorbing boundary at $z=40 \mathrm{~cm}$ is more significant as elastic collision cross-section increases, as it affects $f_{i}(v<0)$, relevant for high intensity elastic exchange, easily appreciated in the temperature evolution close to the domain 
edge. So, elastic collisions are responsible of ion relaxation along the plume axis, leading to the wide ion energy distribution functions found in experimental results.

It is important to notice that the evolution of the distribution function and, as a consequence, its associated moments, is not constant along the axis direction. This means that the collisions between ions and neutrals require a certain length scale to stabilize. Moreover, boundary conditions at the end of the domain also seems to influence the thruster plume, specially for high rates of ion-neutral exchange.

A substantial difference respect to the experimental case is found, as here the accelerated population holds in all the plume and do not vanishes. This could be caused by two main effects not accounted for in this preliminary model: the recombination with electrons or the three-dimensional space in which the relaxation processes take place. As the RPA is only capable of measuring the ions travelling parallel to $z$ those ions that scatter in the radial direction ar not measured, which could explain the reduction of ion density presented in figure 7.2c. Further analysis of these two phenomena is required in order to obtain a better description of the thruster plume.

\subsubsection{Effect of the initial ion velocity}

To discuss the evolution of ions in the thruster plume at different operation regimes, some situations for a wide range of $K_{0}$ are numerically studied now. This value determines the kinetic energy of the ions at the first slab, and it is directly related to the thrust delivered by the electric thruster. With this analysis, we aim to find different behaviours as a function of the energy provided to the ions by the thruster.

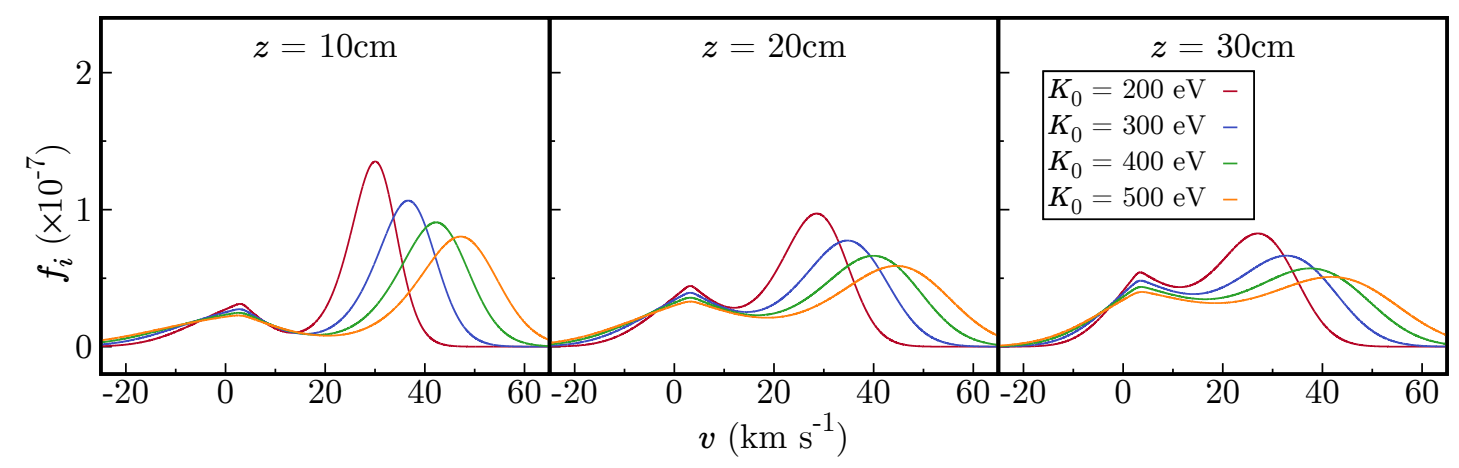

Figure 7.7: Distribution function for different values of $K_{0}$. As initial velocity of ions increases, a more energetic ion flow is delivered by the thruster.

To complete the model, some parameters are required: the Lampe charge-exchange cross-section from equation (7.3) and $\sigma^{i 0}=10^{-18} \mathrm{~m}^{2}$ for the elastic collisions are used, as these parameters seem to produce evolutions close to the experimental data. Here, several kinetic energies are employed $\left(K_{0}=200,300,400\right.$ and $\left.500 \mathrm{eV}\right)$, representing typical values of the ALPHIE operation range. Figure 7.7 plots the ion distribution function at three positions in the plume. The accelerated peak moves to faster velocities as $K_{0}$ increases, while the thermal peak remains at the same position as it is only 


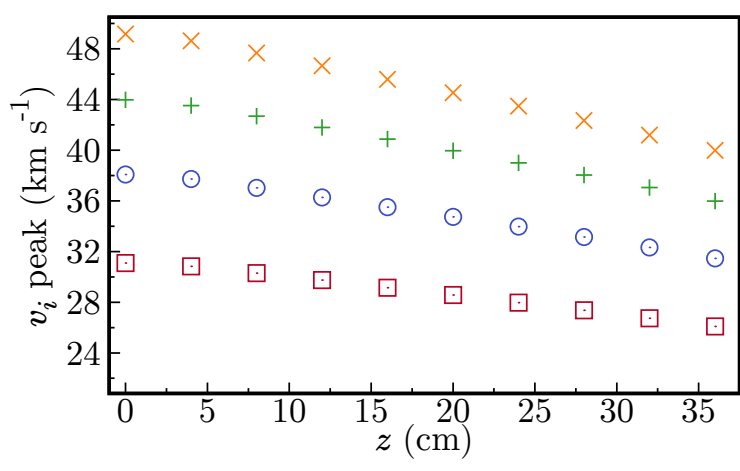

(a) Accelerated maxima

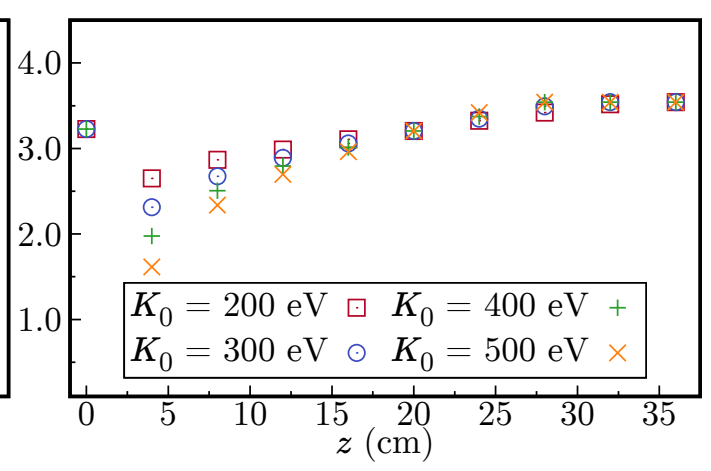

(b) Thermal maxima

Figure 7.8: Evolution of accelerated (a) and thermal (b) ion distribution function maxima $v_{i}$ as a function of $K_{0}$. Values at $z=0$ represent the velocity of the initial ion distribution and the neutral distribution function peak, respectively. The formation of the thermal population is clearly appreciated.

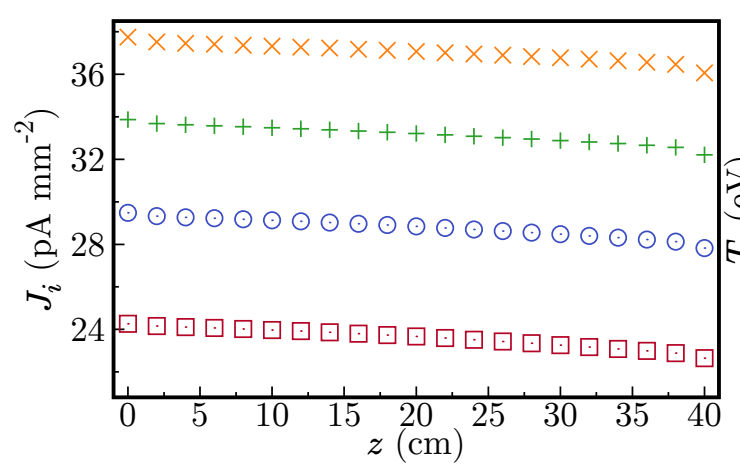

(a) Ion current density

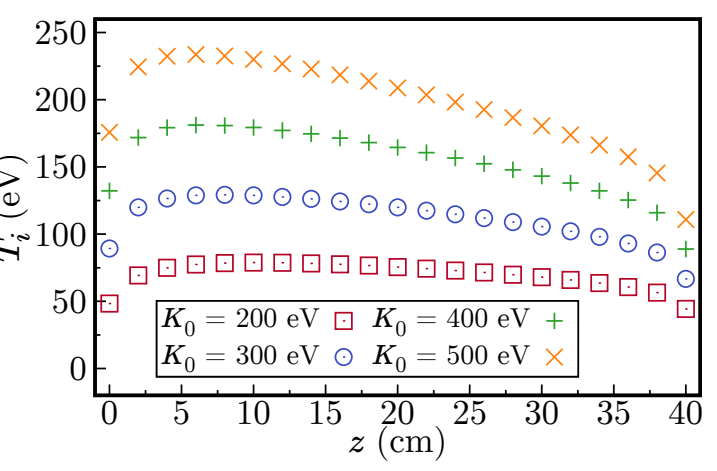

(b) Ion temperature

Figure 7.9: Evolution of the ion current density and temperature as a function of $K_{0}$. A quasi-linear behaviour is found for both macroscopic values as ion initial energy increases.

affected by the neutral distribution function. It is clear that increasing the first slab ion kinetic energy results in a more energetic ion flow, as the peak of accelerated ions increase its velocity. Moreover, ion relaxation also seems to be highly affected by ion energy, while thermal population remains more or less constant, but it seems slightly higher for lower values of $K_{0}$, as found in the experimental results from figure 7.2a. The evolution of distribution peaks, the accelerated and the thermal one, is studied in figure 7.8. The accelerated maximum, figure $7.8 \mathrm{a}$, responds linearly to $K_{0}$, but the peaks velocity reduces as position increase due to elastic exchanges with neutrals. However, the thermal peak, figure $7.8 \mathrm{~b}$, remains almost constant and it is weakly dependent with the ions initial energy. Nevertheless, it can be seen how ions require some length to form the thermal peak and achieve a velocity close to the neutral mean one pictured at $z=0$.

Furthermore, if the ion current density and temperature evolution is analysed through the plume, figure 7.9, it can be seen how an almost linear dependence is found as the initial kinetic energy $K_{0}$ increases. For higher initial velocities, a more energetic 


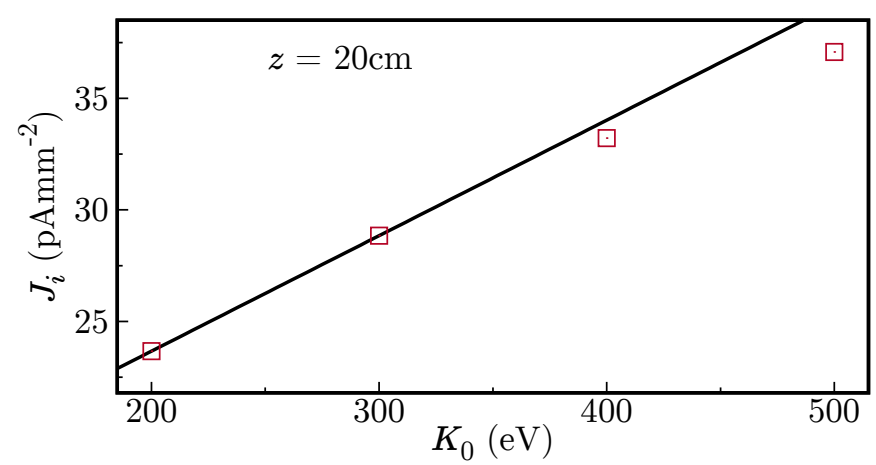

Figure 7.10: Ion current density as a function of $K_{0}$ at $20 \mathrm{~cm}$. Black solid line represents the linear interpolation between $K_{0}=200$ and $300 \mathrm{eV}$.

flow is found, i.e., the ion current density and temperature increase. Nevertheless, while the evolution is quite similar for all $K_{0}$ studied here the increment of $J_{i}$ is not completely linear with $K_{0}$, as can be seen in figure 7.10. For high kinetic energies, ion current density deviates from the linear interpolation computed with the values of $J_{i}$ at $K_{0}=200$ and $300 \mathrm{eV}$. This is related with the increment of ion neutral elastic exchanges as the temperature increases, which results in an intense coupled influence between ions and the neutral background.

\subsection{Conclusions}

The characterization of a thruster plume is of paramount importance to analyse the operation regime of ion thrusters and plan future modifications for these propulsive systems in general, and the actual ALPHIE prototype in particular. In this chapter, a kinetic approach of the ions generated by an electric thruster has been presented accounting for two simple collision interaction: charge-exchange and elastic collisions, which are critical to obtain distribution functions comparable to those measured in the ALPHIE plasma thruster, as the parametric analysis performed demonstrates.

The charge-exchange between ions and neutrals is responsible of the two-peaked distribution function. This means that this process is the main cause to produced the thermal peak, i.e., the ions that appear at the neutral energy. Moreover, this process reduces energy from the ion flux, transmitted to the neutral species as a significant reduction of the current density appears as a consequence of the new thermal ion population. However, ion-neutral elastic exchange creates a relaxation process as wider distributions appear along the axis, reducing the current density and increasing the ion temperature.

If the initial velocity of ions is enhanced the plume becomes more energetic, increasing the ion current density and temperature in an almost linear way. Despite this, for higher values of $K_{0}$, ion population exchange more energy with neutrals, resulting in a less energetic flow. Thence, a non-monotonic response may appear, which could impact 
the thruster efficiency which has to be further analysed.

This model picks some of the basic dynamics that are found in the thruster plume experimental characterisation. Still, this model allows the analysis of a huge variety of scenarios, as parameters as the initial energy of ions, the ionization ratio $r_{i}$ among others; can be modified to obtain a parametric analysis. Also, the capability of the Propagator Integral Method to reproduce physical situations by just evolving a system based on the relevant microscopic phenomena is, again, of huge importance to produce trustworthy results able to cross-check with experiments. 



\section{CHAPTER 8}

\section{CONCLUSIONS}

In the development of this dissertation, different topics from numerical, theoretical and experimental fields have been discussed. Special attention has been paid to their applications in Plasma Physics.

In chapter 2, a generic procedure to apply the Propagator Integral Method (PIM) to general differential equations has been introduced, expanding the applicability of this method to solve a variety of equations that appears in many areas of mathematics, physics and engineering. In addition, a series of numerical hindrances encounter during its development have been presented, to explore the issues that may arise during the resolution of more complex problems. The method provides a stable, accurate and consistent time-evolving solution for all the basic examples presented, but, as all the numerical methods, errors are always introduced. To apply the numerical method to more involved models, it is important to be aware of these errors and try to reduce them. In addition, the method has been applied for the first time to non-diffusive an ordinary differential equations, providing solutions impossible to obtain with classical methods based on finite differences. This extension allows a resolution of other problems in Plasma Physics as the Vlasov or Poisson's equations, without loosing generality or any of the properties presented in the application to drift-diffusion equations. This proves that the time advancing scheme does not relay on the propagator shape and the arduous task is to find a propagator that fulfils the differential problem, with proper boundary conditions.

A brief practical review of kinetic and fluid descriptions of plasmas have been presented in chapter 3, to establish the foundation of terminology and concepts employed in this dissertation. Some examples of evolution of non-collisional plasmas has been presented to test the 1D1V non-diffusive propagator. Relevant dynamics that reproduce physical 
behaviours have been found, specially when a self-consistent electric field is coupled with the charged species dynamics to account for the possible charge separation. Differences between the heavy and light species due to the action of this electric field arise naturally as a consequence of the self-consistent model utilised. These scenarios require a further exploration, because they could be relevant to analyse typical phenomena of non-collisional plasmas, as the Landau damping. Thence, a new path to the analysis of non-collisional plasmas by means of the Propagator Integral Method has been opened.

Chapter 4 has introduced the collision terms and other particle interaction process employed through this work. Numerical conservation of norm, momentum and energy for one and two disparate species has been tested, finding that the PIM is an excellent tool to deal with these problems and conserve the macroscopic quantities with very small numerical errors. In addition, a comparison between typical collisional operators has been performed, finding that BGK term can produce non-physical transitory situations that derive in an incorrect entropy evolution.

Chapters 5, 6 and 7 present the main applications of the PIM to the kinetic model developed to analyse different plasma types.

Firstly, chapter 5 presents a new one-dimensional three species (electrons, ions and neutrals) kinetic description for Weakly Ionized plasmas which include all elastic interactions among the species. A huge influence of the charge-neutral interaction has been found, meaning that neutrals have a relevant impact in the dynamics of weakly ionized plasmas and a self-consistent model is required to describe these systems. The model is consistent with plasma theory and accounts for relevant phenomena in the one-dimensional velocity space. Dynamics of all species depend largely in the collision frequencies, which change with the species energies and densities. This becomes relevant in may situations that appears naturally, e.g., when an electric field changes abruptly. Beside the different test performed to analyse the response of a WIP in a variety of scenarios, this model has been used to study a discrepancy found in experiments when a Langmuir probe was utilized to characterise a plasma. Numerical and experimental analysis indicates that the change in plasma response to different sweep frequencies is related to variations in the the exchange-rate between charged and neutrals due to fast changes of the electric field that leads to variations in temperature. In addition, a calculation of transport coefficient for electrons as a function of electric field and the ionization ratio has been performed, finding a similar response as the one-dimensional model. Linear evolution of electron density current and heat flux up to a certain ionization ratio appears where temperature remains constant with the ionization ratio and only depends on the electric field intensity. After a certain ionization degree, electron temperatures drop rapidly as the electron-ion exchange gains relevance. However, the model should be expanded to account for ion and neutral evolutions. Nevertheless, this path of computing transport coefficients by solving the complete distribution function opens a wide range of possibilities and its development will be continued in the future.

The chapter 6 presents two kinetic descriptions for the plasma wall interaction. First 
one deals with the electrons in the wall and in the vacuum chamber as a discontinuous medium. Effect of the wall temperature, background pressure and electric field have been analysed. It has been found that electron-neutral interaction is of mayor relevance as it reduces the energy of the emitted electrons and acts as an additional barrier to the emission process. The influence of the self-consistent electric field generated was also analysed. The effect of this force depends on the potential difference between the wall and the vacuum chamber, as it may create a potential well that retains a population of emitted electrons or may saturate the emitted density current for certain values of wall potential. The variation of the potential well and the saturation phenomena as a function of the wall temperature and background pressure was studied. The saturation current was proven to be constant regardless the neutral density in the vacuum chamber. However, the potential difference required to reach it depends on the neutrals pressure and the wall temperature. In addition, a model to describe the collection of charged particles, electrons and ions, in front of a planar wall was presented. Influence of the ions dynamics and the charge-neutral collision intensity was studied, two phenomena that are usually neglected in similar models. Because ions are also collected by the wall, which reduces its density deriving in a significant variation in the self-consistent electric field if this species evolution is not accounted for. This influences the electron dynamics, specially the quasi-steady value reached. Charge-neutral collisions result relevant as electrons and ions dynamics are affected by the intensity of this exchange, affecting transitory and steady responses. Further analysis of both plasma-wall models are required, specially the translation of these models to cylindrical and spherical coordinates to match the common geometries of probes employed in the laboratory.

Finally, chapter 7 , presents an analysis of a ion thruster plume by means of a pure kinetic model. The main phenomena that generates the ion distribution functions found by experimental means were analysed. The charge-exchange between high energy ions and low energy neutrals is the main reason for the thermal peak to appear, while elastic collisions result in a relaxation of the ion population, allowing them to have a wide range of velocities between the thermal and accelerated ones. The two processes studied are mandatory to obtain results similar to those found in the laboratory. For increasing ion initial velocity, the resulting plume becomes more energetic, but a linear evolution in the ion density current is not found for high velocities, meaning that an important part of the ions initial energy is being transferred to the neutral species. In all cases, the PIM efficiency and sensibility to detect non-linear effects has been stated as an useful tool for the present and future works.

\subsection{Future lines of research}

The development of the numerical method and its application to solve involved kinetic problems opens new possibilities to obtain pure kinetic, non-linearised, descriptions of plasmas. Of course, this means that new research possibilities arise in numerical and 
theoretical frames to expand the applicability of the numerical method.

The main topics of the PIM that should be improved are:

- Reducing the memory required to store the propagator and the time per iteration. As we saw, the PIM requires large amounts of memory (RAM) to store the propagator and a long time to compute each time step. These problems should be addressed to increase the number of points in the grids and allow longer simulation times.

- Performing a distributed simulation to allocate resources from different nodes in a cluster. This will allow the inclusion of more grid points in the simulations, which would specially improve the resolution of multiple-dimensional problems. Thence a method to separate a simulation across different nodes in a cluster machine employing Message Passing Interface (MPI) should be developed.

- The improvement of the solution stability and stability when the diffusive tensor is small, specially in $1 \mathrm{D} 1 \mathrm{~V}$ cases.

- Conservation for two-velocity collision terms should be improved to reach an error in the order of $10^{-10}$, as in the one-dimensional cases.

Moreover, new revisions of the models studied in this dissertation should be developed to account, among others, for:

- Transport coefficients computed in chapter 5 should be extended to account for ion and neutral evolutions, as well to extend the computations to other gases commonly employed in laboratory and commercial applications. Calculations presented here open a new path to compute transport coefficients in a variety of situations that should be explored in future works, to obtain a data base suitable for fluid models.

- Discontinuous plasma-wall kinetic model presented in chapter 6 should be improved to account for a more physical representation of the wall, as well as the coupled electric field, which should penetrate into the metal. Also, the geometry of this model and the one presented to analyse the collection of charges should be translated into cylindrical and spherical geometries, respectively, to better match the usual probe shapes employed in experimental setups.

- To improve the description of the thruster plume presented in chapter 7 , the effect of electrons and the self-consistent electric field should be included, as well as account for more complex representation of interactions among particles to better represent the wide range of energy for which ions appear.

In addition, some cross-checks with experiments are required to corroborate some of the conclusions reached in this dissertations. A collaboration with the experimental 
team of the UPM PlasmaLab should be carried out in order to address the following topics:

- Perform a measurement of plasma parameters by means of the floating potential method to cross-check with I-V curves obtained by a Langmuir probe and numerical results presented in section 5.2. This will helps in the election of a sweep signal to provide the right plasma parameters.

- Improve the analysis of transitory regimes in measurements, as they may impact the quasi-steady regimes, specially in those involving the biasing of probes.

- Explore the influence of collisional process and heavy species dynamics in the plasma diagnosis.

- Analysis of the Retarding Potential Analyser probe by means of numerical methods to fully understand the dynamics of ions and electrons inside it and improve its further design.

In a more general way, an effort should be make to apply the progress related with the PIM obtained here to other branches of physics, mathematics and engineering. 



\section{COLLISIONS IN PARTICLE-IN-CELL CODES WITH PROPAGATOR INTEGRAL METHOD}

This appendix resumes the last contribution in this dissertation, with the aim of extend the applicability of the PIM to other, well established fields. Particle-in-cell (PIC) codes are widely employed for the simulation of plasma dynamics $[12-15,163]$. In these numerical approaches, the kinematic equations of a large number of macro-particles, which may represent a huge amount of real particles, are solved to obtain the transient position of each particle, based on the electromagnetic force action and the possible boundary conditions involved in the problem. The charge density, electric current and any other mesoscopic property of the particle ensemble are placed into the vertices of a set of fixed cells that meshes the problem position domain. The coupled selfconsistent electromagnetic field is calculated at these fixed cells and applied to the system dynamics in the following iteration of the simulation. A major limitation in these methods arises due to the difficulty to ascertain an appropriate inclusion of interaction processes among the particles. Direct improved calculations accounting for relevant microscopic effects held among particles require an amount of computational resources that is unaffordable for modern computations in most cases. Under this view, Monte Carlo Collision (MCC) methods are usually introduced in PIC simulations to account for inter-particle collisions $[12,17,164]$. These models essentially compute the probability of interaction based upon cross-sections data to analyse the scattering phenomena. Hence, this approach assumes that a collision occurs, as a random process of pure mathematical nature.

In this appendix, we present a new approach, which is not strictly related to Monte Carlo implementations, to implicitly include collisional scattering into PIC codes. This new method is founded on taking advantage of the theory of kinetic collisional processes. Therefore, for each macro-particle, the scattering due to collisional effects is computed 
thanks to a short-time transition probability function $P_{\tau}$ in velocity space, constructed by extracting information of theoretical drift-diffusion equations representing collisional processes. To include collisional effects in a PIC code with $P_{\tau}$, it is proposed to use the Propagator Integral Method to construct an auxiliary distribution function for colliding particles. As we introduced in chapter 4, this method allows the calculation of the complete distribution function accounting for system collective effects due to collisions. Then, the particle post-collisional velocity is randomly assigned, but the probability of each post-collisional velocity is weighted by its corresponding distribution function value. This procedure, in addition to the action of the electromagnetic force over the particle, provides the macro-particle's final velocity, employed to obtain the position at the next time step. Consequently, with this approach, particle collisions are treated as a result of collective effects. This physically meaningful approach allows the incorporation of complex differo-integral terms, coming from kinetic theoretical analysis in a self-consistent manner. This is not based, as other approaches, on the us of Langevin equations $[16,165]$, but on their associated probability transition functions.

\section{A.1 The weighted random scattering based on the distri- bution function}

The basic procedure to obtain the macroscopic post-collisional velocity by means of the PIM is presented as follows. To simplify the study and for sake of clarity, this work focuses on the analysis of a one-dimensional motion (1D1V). However, this procedure can be easily extended to higher dimensional problems. Hence, following the usual theoretical techniques taken from the kinetic theory standards, a particle of velocity $v^{\prime}$ at time $t^{\prime}$ reaches velocity $v$ at time $t>t^{\prime}$ due to collisional effects. This effect can be described using probability concepts grounded on the physical stochastic nature of the collisional problem. In this sense, a short-time transition probability function $P_{\tau}$ is constructed as

$$
P_{\tau}\left(v, v^{\prime}\right)=\frac{1}{\sqrt{4 \pi D_{v v}^{\prime} \tau}} \exp \left[-\frac{\left(v-v^{\prime}-D_{v}^{\prime} \tau\right)^{2}}{4 D_{v v}^{\prime} \tau}\right],
$$

following the procedure show in chapter 2. This function represents the probability that a particle with velocity $v^{\prime}$ takes the value $v$ in a short time $\tau=t-t^{\prime}>0$, based upon the convective and diffusive parameters $D_{v}^{\prime}$ and $D_{v v}^{\prime}$ evaluated at $v^{\prime}$ and $t^{\prime}$. These two parameters can be as complex as necessary to properly represent the collisional processes felt by the macro-particle of velocity $v^{\prime}$ due to the presence of the other particles.

It is apparent that the width of the Gaussian transition probability function (A.1) is related to the diffusive scale, defined by $D_{v v}^{\prime} \tau$, and that the function maximum value is reached at $v=v^{\prime}+D_{v}^{\prime} \tau$. To represent the probability function of each macro-particle, a self-adjustment of the numerical grid is performed as follows. First the grid length 
is computed as

$$
L_{v}=2 \sqrt{-4 D_{v v}^{\prime} \tau \ln \left(\epsilon \sqrt{4 D_{v v}^{\prime} \pi \tau}\right)}
$$

where $\epsilon$, in units of $\Delta v^{-1}$, is a very small number to computationally limit the extension of the Gaussian function, provided that $0<\epsilon \sqrt{4 D_{v v}^{\prime} \pi \tau}<1$ is satisfied. In all calculations presented in this appendix, $\epsilon=10^{-12}$ to ensure that the transition probability tails are properly represented, but much larger values of this parameter have been tested and no influence in the system dynamics has been found. Secondly, the grid is centred at the point $v^{\prime}+D_{v}^{\prime} \tau$, to ensure that the maximum value of the Gaussian function is always captured, i.e., it consistently lays on one grid point.

Now, it is assumed, as a benchmark problem to introduce the method, that particle's velocity $v^{\prime}$ is modified by the interaction with a fixed background of particles with zero mean velocity and constant temperature. This dynamic corresponds to the wellknown Brownian motion $[61,64,65]$, where the particle's dynamic is subjected to two phenomena: convection, due to the drifting force of the form $D_{v}^{\prime}=-\gamma v^{\prime}$ and the diffusive effects described by $D_{v v}^{\prime}=\gamma T$ in dimensionless units, where $\gamma$ is a positive constant as a collision frequency, being $T$ the temperature of the fixed background. The probability function (A.1) is therefore used to obtain the auxiliary distribution function $g(v)$, that encloses the physics of the collisional problem thanks to $D_{v}^{\prime}$ and $D_{v v}^{\prime}$, as

$$
g(v)=\int \delta\left(v^{\prime}-v^{\prime \prime}\right) P_{\tau}\left(v, v^{\prime \prime}\right) \mathrm{d} v^{\prime \prime}=\frac{1}{\sqrt{4 \pi \gamma T \tau}} \exp \left[-\frac{\left(v-v^{\prime}+\gamma v^{\prime} \tau\right)^{2}}{4 \gamma T \tau}\right]
$$

where $v^{\prime \prime}$ is a dummy variable and $\delta$ is the Dirac delta. Since each particle has different $v^{\prime}$, the resulting convective and diffusive parameters for each one may differ. This means that the function $g(v)$ and the grid to represent it must be computed for each macro-particle. To properly depict $P_{\tau}$, only a few grid points are required thanks to the self-adaptation of the grid presented above, a fact that reduces the time required to compute the auxiliary distribution function. This integral, in practice, acts as a filter to provide the function $g(v)$ from $P_{\tau}$, which becomes the support to construct the weighted probability function, used to assign the final post-collisional velocity as it is explained below.

In an usual PIC code, a particle with velocity $v^{\prime}$ requires a unique post-collisional velocity to compute the position at the next time step. In this sense, it becomes inevitable to randomly assign a unique post-collisional velocity $v$ to each macro-particle inspired by the function $g(v)$ computed previously. To do this, $g(v)$ is used to generate the weighted probability for each post collision velocity $v$ to occur. The procedure takes advantage of the probabilistic meaning of $g$ and its operates as follows: firstly, 


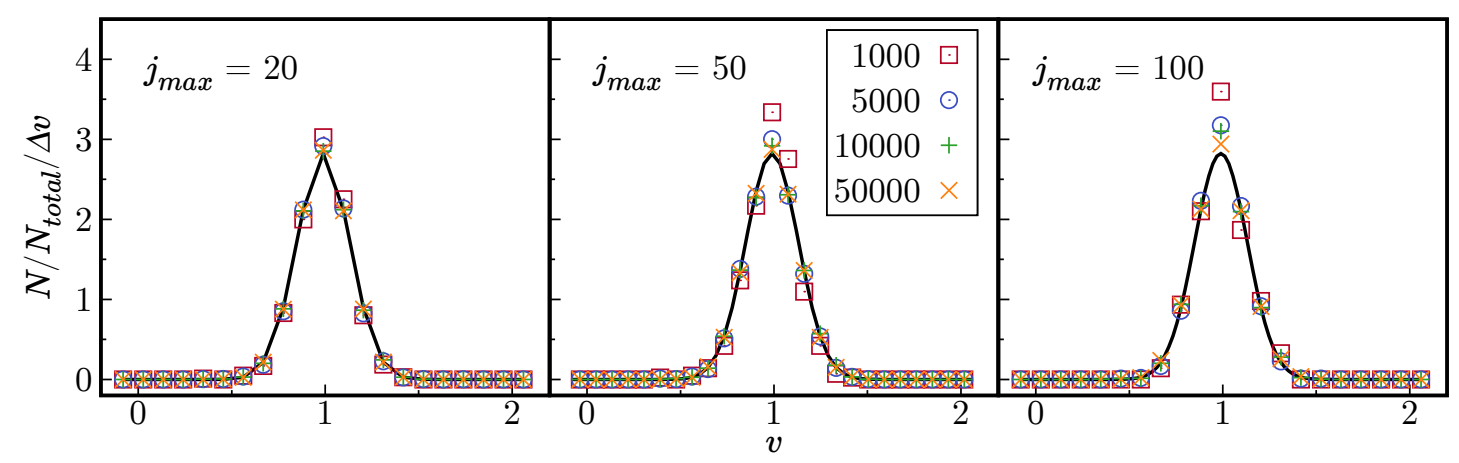

Figure A.1: Weighted distribution of random particles as a function of $N_{\text {total }}$ and $j_{\max }$. Function $f_{j}$ is represented as a black solid line.

the cumulative sum of $g(v)$ is computed for each point in the grid $v_{j}$ as

$$
C_{j}=\frac{\sum_{j=0}^{j} g_{j}}{\sum_{j=0}^{j_{\max }} g_{j}}
$$

where the subscript $j$ represents a point in the grid, $g_{j}$ is the function $g(v)$ evaluated at $v_{j}$ and $j_{\text {max }}$ is the maximum number of grid points employed to represent $g(v)$. This $C_{j}$ is ranged between 0 and 1 , and it represents, in fact, the cumulative probability for each post-collisional velocity $v_{j}$. Once this sum is computed, a random number between 0 and 1 is generated. This random number is related to the closets value of $C_{j}$. The corresponding $v_{j}$ is assigned to the particle as the post-collisional velocity. In resume, this procedure replaces the usual implementation of Monte Carlo Collisions in PIC codes by weighting the post-collisional velocity probability with a physically meaningful distribution function, i.e., based on the collective effect of other, or the same, species, thanks to the action of convective and diffusive phenomena.

For the case of $D_{v}^{\prime}$ and $D_{v v}^{\prime}$ presented above, the post collision distribution function $g_{j}$ is now computed for different values of $j_{\max }$ and different number of total particles, all with the same $v^{\prime}$. The post-collision velocity is randomly assigned following the procedure stated above. For this example, $v^{\prime}=1, \gamma=1, T=1$ and $\tau=10^{-2}$ is employed to obtain $g_{j}$. Figure A.1 shows the numerical function $g_{j}$ as a black solid line and the number of particles in each after collision velocity for $N_{\text {total }}=1000,5000$, 10000 and 50000 test particles and $j_{\max }=20,50$ and 100 grid points are pictured with symbols. The distributions of test particles are normalized by $N_{\text {total }}$ and by the distance between grid points $\Delta v=v_{j+1}-v_{j}$ for each case. It can be easily seen how the parameter $\epsilon$, used as a cut-off criteria to calculate $L_{v}$, is small enough to depict $g_{j}$. This ensures that no particle is assigned to the largest velocities, far from the Gaussian maximum value, proving that $\epsilon$ could be much larger to improve the representation of $g_{j}$ keeping the same amount of grid points. Since the maximum number of grid points available to represent the auxiliary distribution function increases, $g_{j}$ becomes quite closer to a Maxwellian distribution. However, more particles are required to represent 
the appropriate values of this function. In all cases, it can be seen how most of the particles end around the point $v^{\prime}+D_{v}^{\prime} \tau$ and that the shape obtained by the distribution of a large number of particles always reassembles a Maxwellian distribution.

\section{A.2 A practical example: a 1D1V Brownian motion}

In this section, a simple one-dimensional PIC code is presented to solve a forced Brownian motion with and without using the procedure explained above to include collisions with a fixed background. The basic scheme to advance the macro-particles in time simply becomes

$$
\begin{aligned}
x_{i}^{n+1} & =x_{i}^{n}+\tau v_{i}^{n+\frac{1}{2}} \\
v_{i}^{n+\frac{1}{2}} & =v_{i}+F_{i} \tau
\end{aligned}
$$

where $F_{i}$ is the external force, per unit of mass, $v_{i}$ is the velocity after the collisional process, with the pre-velocity $v_{i}^{\prime}=v_{i}^{n-\frac{1}{2}}$, subscript $i$ refers to each individual macroparticle. Here, $n$ is the current iteration and $\tau$ is the time step. The deterministic dimensionless force in this benchmark case is $F_{i}=-x_{i}^{n}$, which produces a periodical evolution of the particles, regardless their initial condition. When collisions are not accounted for, $v_{i}=v_{i}^{\prime}=v_{i}^{n-\frac{1}{2}}$ is used to recover the classic non-collisional PIC advancing scheme. At each time step, the particles are weighted inside 200 fixed cells, equally spaced, in a grid that covers the non-dimensional distant from -1.5 to 1.5. Using this grid, the non-dimensional particle density $n(x)$ is obtained. This density results as an equivalent of having integrated the distribution function $f(x, v)$ over the velocity space as $n(x)=\int f(x, v) \mathrm{d} v$.

In this example, 50000 particles with random distributed initial positions and velocities, lying between -0.1 and 0.1 are used. The maximum number of grid points to model the auxiliary distribution function, $j_{\max }$, is 50 . This ensures an accurate representation of the auxiliary distribution function $g_{j}$ with the selected number of particles. Three different cases are therefore studied: a collisionless case and two collisional ones, with $T=0.1$ and $T=0.01$, with $\gamma=1$. The time step $\tau=10^{-2}$ is employed in these simulations to advance the particle motions and compute the post-collisional velocities when required.

The position and velocity of particles at different time frames are pictured in figure A.2. Figure A.2a presents the collissionless case. It can be seen how the initial package of particles remain unaltered and only the rigid rotation, found in previous examples with similar external acceleration, appears. The inclusion of collisions produce a scattering of particles in the $x-v$ domain, as can be seen in figure A.2b and A.2c. As temperature increases, the velocity of particles also does as an effect of the random scattering. For the case of $T=0.1$, velocities and positions in the order of $\sim 1$ are reached. The effects 


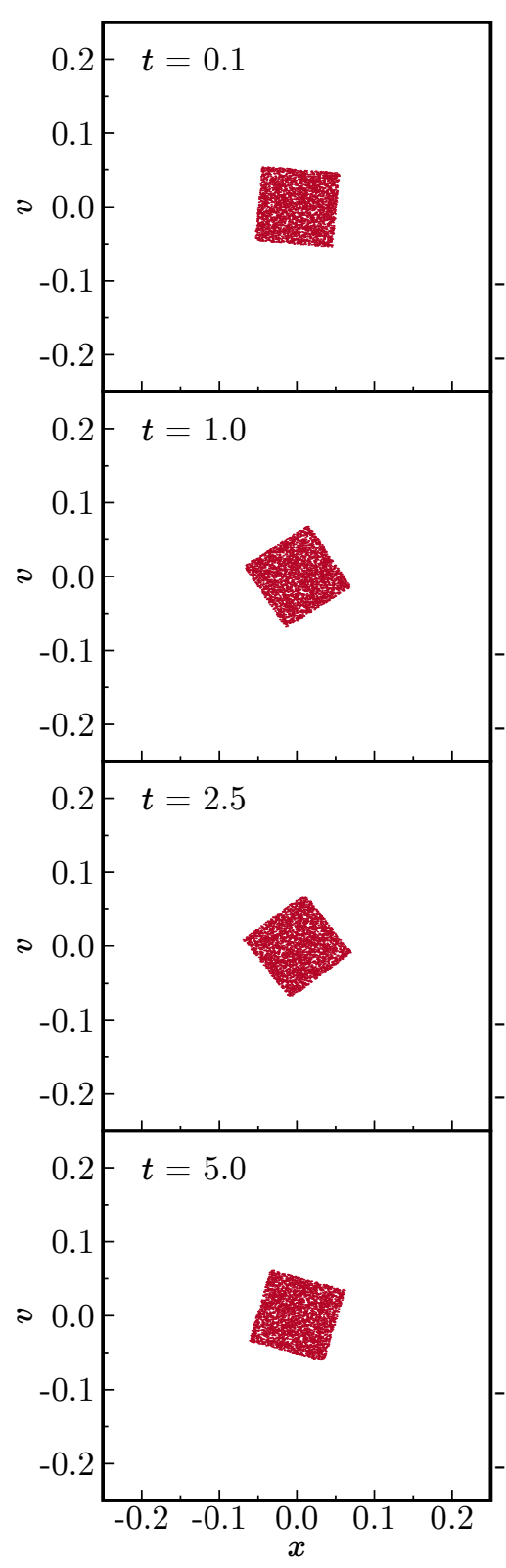

(a) No collisional

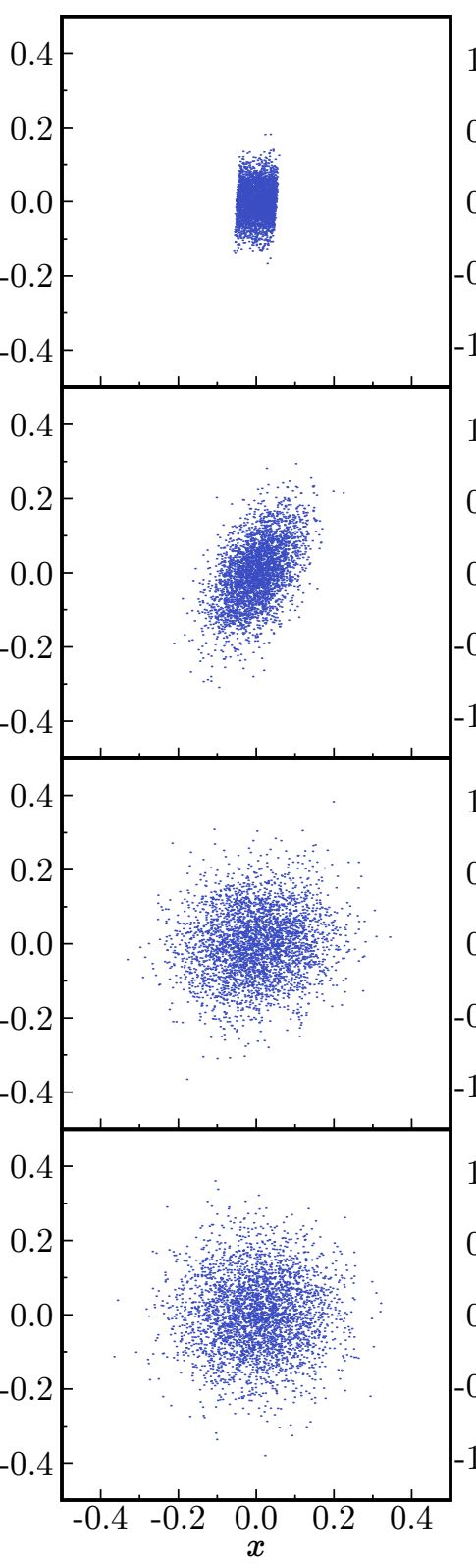

(b) $T=0.01$

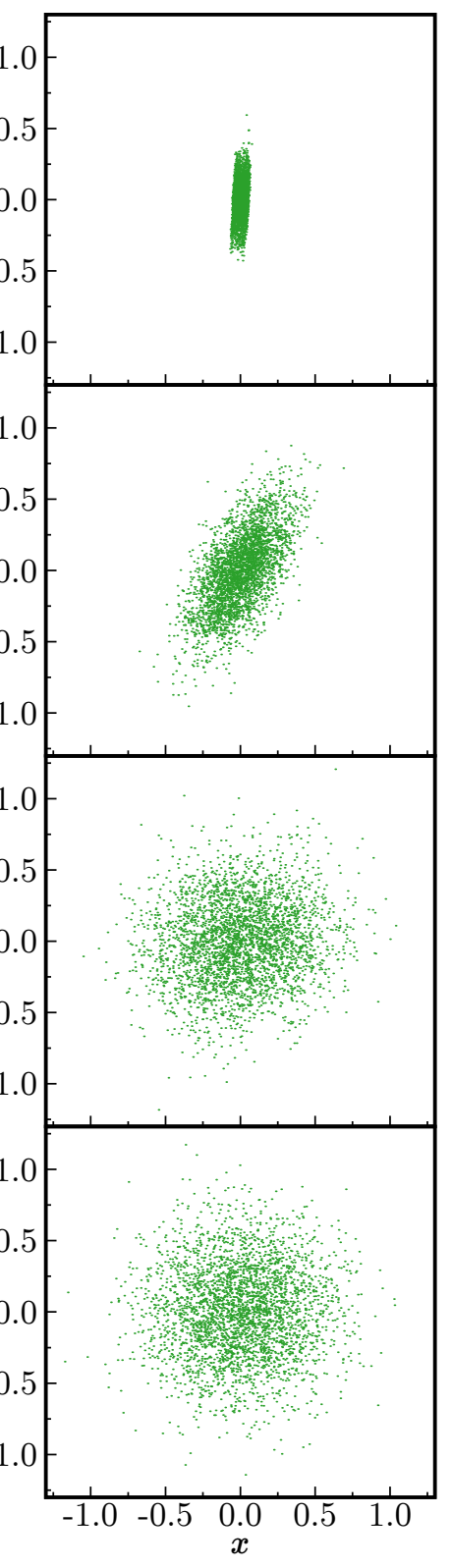

(c) $T=0.1$

Figure A.2: Evolution of particles in the $x-v$ domain for different scenarios: (a) Non-

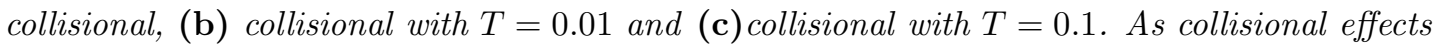
increase, a more distributed particles are found, reaching higher velocities and positions. 


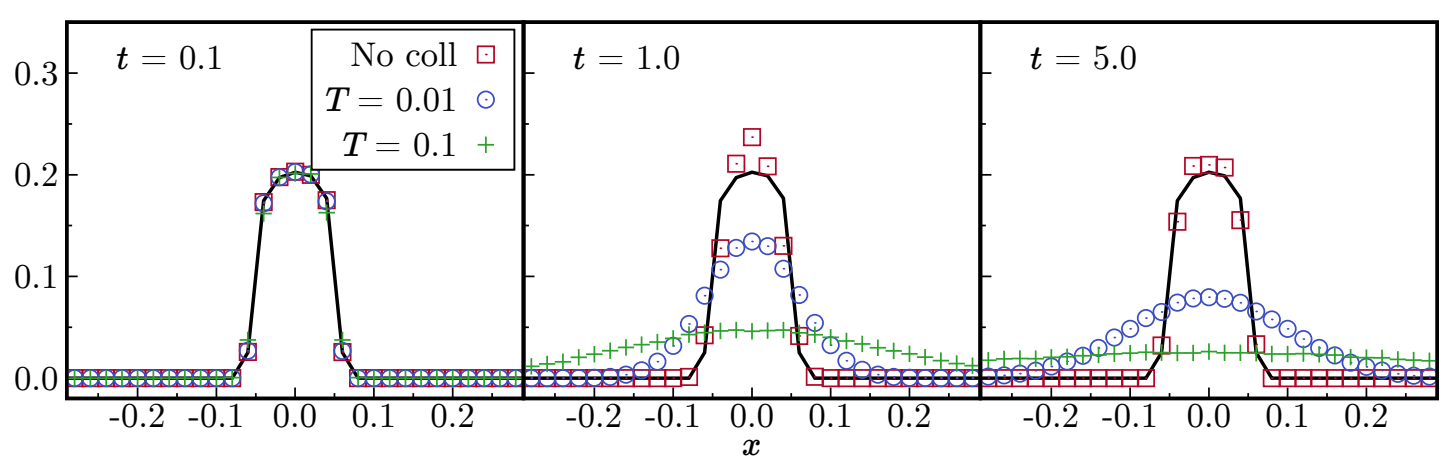

Figure A.3: Time evolution of density for the three solved problems. Collisions smooth the distribution of density and also scatter particles at high values of $v$, which results in a wider distribution of density. Initial condition is represented with a black solid line.

of collisions also lead the system into a homogeneous situations, where all directions in the $x-v$ plane has a similar amount of particles, regardless the initial condition. Also, the effect of the collisions can be appreciated as time advance. For very short times, top row of figure A.2, particles still retain some of the initial shape, but for $t=2.5$ nondimensional units both collisional solutions present a particle distribution that almost match the final state.

The distribution of density, in dimensionless units, $n(x)$ computed at the fixed cells is shown in Fig. A.3. At $t=0.1$, all three solutions result in a similar distribution of density. As time increases, the effect of collisions becomes more relevant because it produces diffusive effects, giving rise to a Gaussian distribution of density. On the other hand, for the non-collisional case, the initial sharp condition, represented as a black solid line, is recovered at $t \simeq 5$. The width of the density distribution in the collisional cases depends on the fixed background temperature, as expected. For increasing temperatures, particles are scattered at higher velocities, leading to a wider profile of distribution of density. The self-adjustment of the grid to numerically represent $g(v)$ allows the incorporation of low and high intensity collisional effects, with the same number of grid points. This facilitates the implementation of complex collisional phenomena in a self-consistent way, that may evolve at different time scales or intensities.

\section{A.3 A non-linear example, the Kappa 1D1V motion}

In addition, non-trivial drift-diffusive effects can be incorporated into PIC simulations with the method presented in this appendix. For example, let us assume now that the convection-diffusive coefficients for our one-dimensional problem are

$$
D_{v}=-v ; D_{v v}=1+\frac{v^{2}}{2 \kappa}
$$




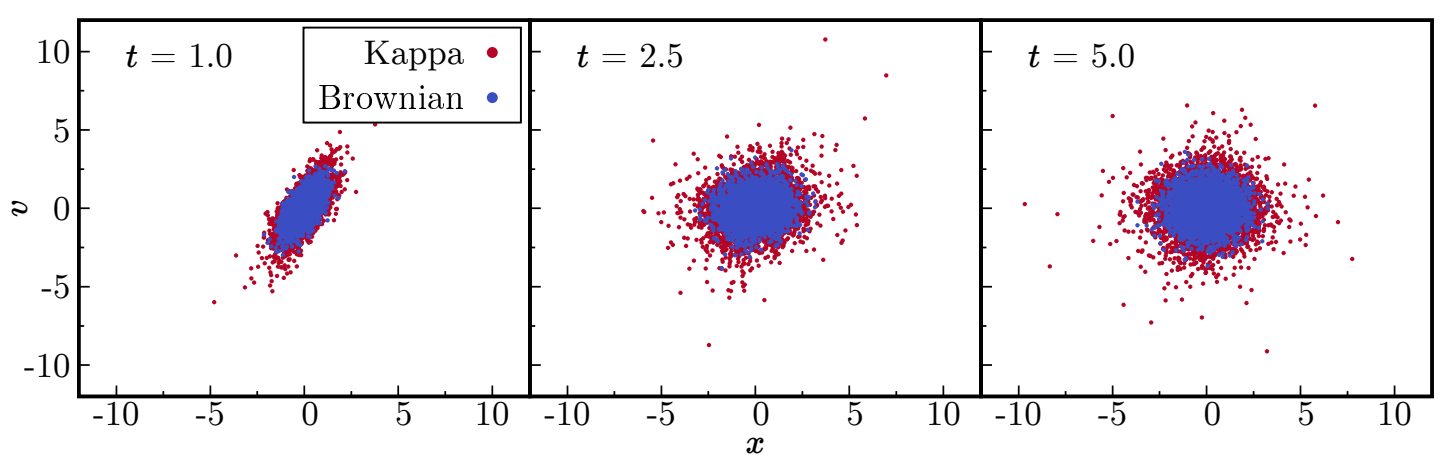

Figure A.4: Evolution of two different drift-diffusive effects: a $\kappa$ (red dots) and Brownian (blue dots) motions. Effect of the $\kappa$-like operator allows particles to reach higher velocities, which will translate in high energy tails in the distribution function.

which will represent the well known kappa problem presented, for example, as a testcase in section 2.2.1. As we saw in previous examples, these terms produce a high energy tails solution, which translated into our PIC code means that particles can reach higher velocities that in the Brownian motion. For this example, $\kappa=\frac{5}{3}$ and the other parameters (number of particles, number of cells, time step and external force) are leave the same as in the previous example. The evolution of particles under these terms in the $x-v$ plane is compared with a Brownian motion at $T=1$ in figure A.4. It can be seen how a high number of particles reach velocities two times higher that those obtained with the Brownian motion presented above. This means that complex non-linear drift-diffusion parameters can be incorporated into the PIC code by means of the PIM, obtaining a physical evolution that agrees with the parameters passed to the propagator. This will be of mayor importance when, in future works, involved collision terms, where highly non-linear parameters can appear, are implemented.

\section{A.4 Conclusions}

The physical meaning of the short-time propagator has been applied to compute the random scattering of particles in PIC codes due to collisional effects. The implementation of collisional effects has always been a hot topic in the development and evolution of PIC codes. In this appendix, a new method to provide a physically meaningful way to randomly scatter particles supported by the collective effect of collisions has been introduced. This procedure to implement collisions has no relation to classical Monte Carlo Collision methods, although it also introduces scattering of macro-particles by collisional effects. In the model introduced here, this dispersion is based on physical drift-diffusion coefficients computed accounting for the physics of the collisional processes. Due to the physical representation provided by the transition probability $P_{\tau}$, a relative large time step can be employed, up to one percent of the characteristic relaxation time. A simple example, consisting of a one-dimensional Brownian motion with an external linear force that produces a periodical motion of particles, has been presented 
to illustrate the method. Evolutions without collisions and accounting for them with a fixed background of particles at two different temperatures have been compared. It is observed that when the background temperature increases, particles scatter, spreading the distribution function, as expected. Moreover, a $\kappa$-like drift-diffusion problem has been solved, producing a higher dispersion than in a Brownian motion. The probability transition function $P_{\tau}$ can be constructed by taking information of theoretical driftdiffusion equations, as the Fokker-Planck-Landau one describing the Coulombian interaction among charged species. Further analysis to include more complex collision terms will be presented in future works. 



\section{APPENDIX B}

\section{NUMERICAL LISTINGS AND SUBROUTINES EMPLOYED IN THIS WORK}

In this appendix, some of the numerical listings employed through this dissertation to implement the Propagator Integral Method and solve the test cases and model proposed are introduced. All codes developed in this work were written in Fortran 95, but they can be easily extended to other programming languages. This appendix is not intended to be a recompilation of complete codes but some guidelines to explain how some numerical challenges that arose through the implementation of the PIM have been solved. Its main propose is to provide some basic subroutines and justify, from a numerical point of view, some of the decisions taken.

\section{B.1 Computation time reduction with OpenMP}

OpenMP is an API (Application Programming Interface) that supports shared memory multiprocessing programming in a variety of architectures and languages. More information can be found in the general references [166-169] and in the OpenMP project site $^{1}$.

Through this dissertation, OpenMP has been employed to divide long tasks among different threads, reducing the total wall clock time required to compute the time evolving solution. This allows a better employment of the computational resources and the capability to calculate more iterations in less time with more points in the numerical grid.

\footnotetext{
1 http://openmp.org/wp/about-openmp/
} 
In this section, a basic example of time saving thanks to OpenMP is presented. This example was executed in the High Performance Computing (HPC) centre of CeSViMa ${ }^{2}$, where each node consist on two Power7 CPUs of eight cores each at $3.3 \mathrm{GHz}$. The example consist on solving the evolution of a single species distribution function under the effect of self-collisions modelised with a Dougherty term, as the one presented in 4.1.4. An amount of 10001 points and 100 iterations was employed. The main parallelisation performed for this case were in the calculation of the one-dimensional propagator, presented in listing B.1. First line in this listing divides the the double loop in the number of threads specified. The instruction REDUCTION (+:Norm) is required to ensure that the propagator norm is correctly computed.

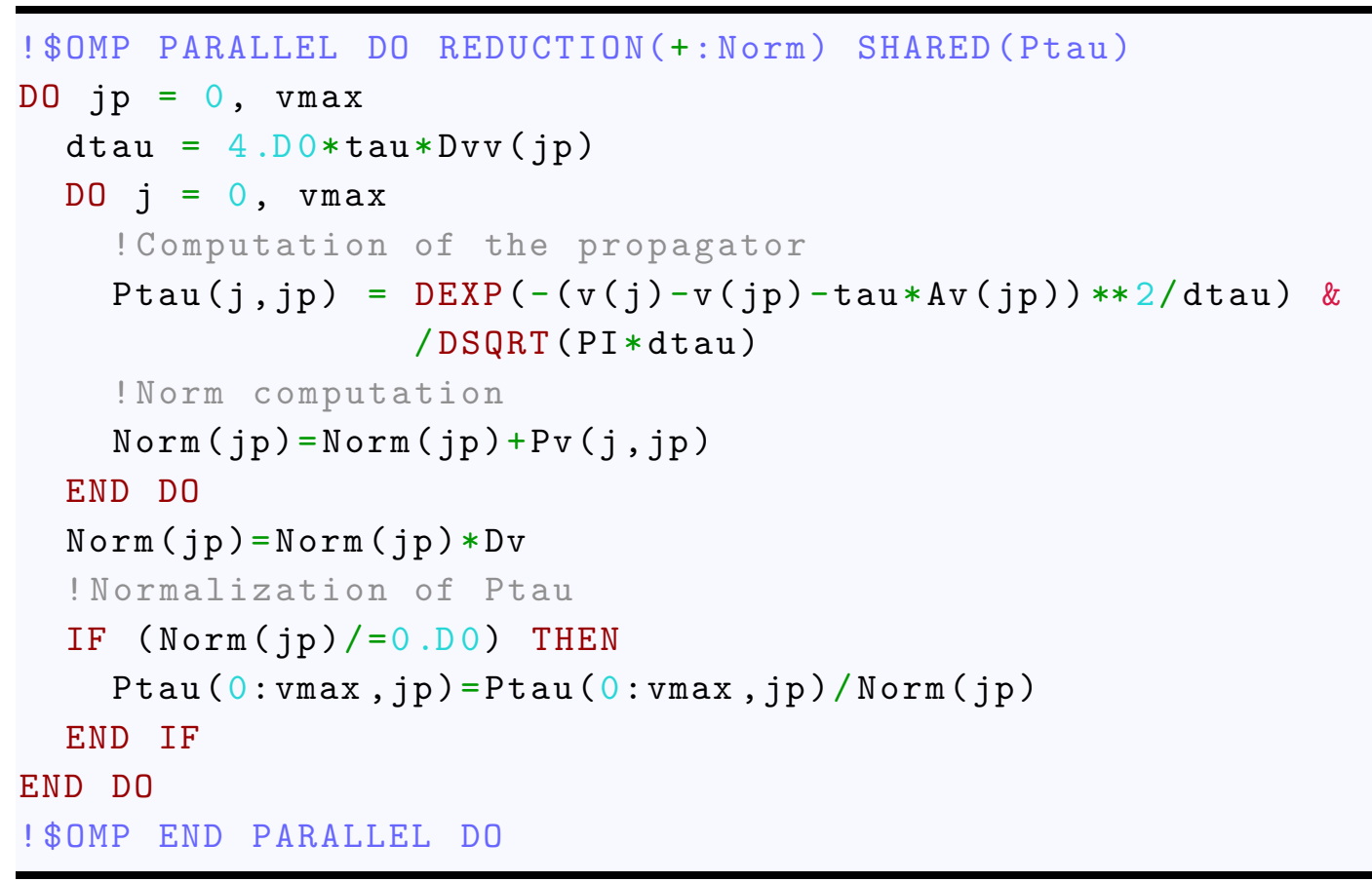

Listing B.1: Reduction of time calculation for the propagator with OpenMP. A proper renormalization of the propagator is performed

To advance the function, the Fortran intrisic MATMUL is employed to multiply the function at the previous time step times the two-dimensional array that contains the propagator. Figure B.1 pictures the computational time required to complete 100 iterations as a function of the number of threads compared with the no parallelisation case (only one thread). A exponential dependency between the computational time and the number of threads is found, meaning that doubling the threads does not always translate in an appreciable reduction of computational time. It is important to remark that the same numerical solution is always reached, regardless the number of threads employed.

2 http://www.cesvima.upm.es/ 


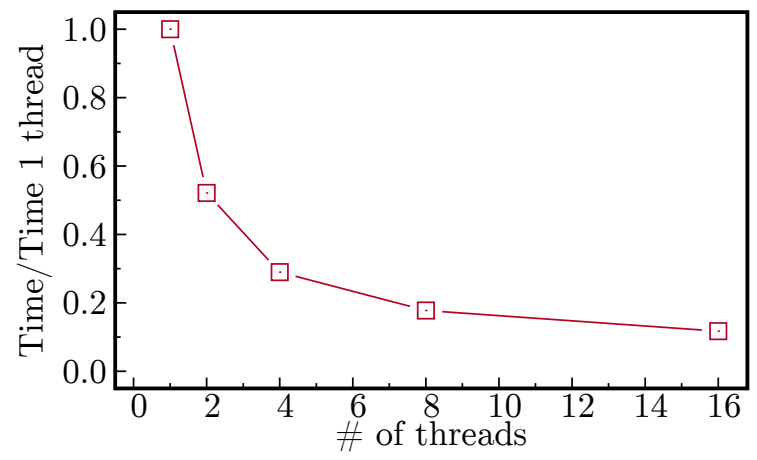

Figure B.1: Computational time as a function of the number of threads. An exponential reduction is found.

\section{B.2 Rectangle integration method}

To perform numerically all the integrals presented in this work, a rectangle integration method was employed. These integrals are mainly used to advance the solution by means of the numerical propagator, to compute the macroscopic quantities and fluxes of the solution and to calculate non-homogeneous terms as charge-exchange. The basic structure of this method can be found in listing B.2. The PIM is not limited to this integration scheme and any other valid method can be utilized, but we have found that this one is easy to implement and always produce a solution that behaves as the analytical one, as test-cases in this dissertation prove. It is important to remark that the integration scheme used should not influence the system evolution as the physics of the problem is enclosed inside the propagator, not the advancing scheme. The numerical integration is just a tool to solve the next step solution, as analytical solutions are impossible to obtain except for some very simple cases.

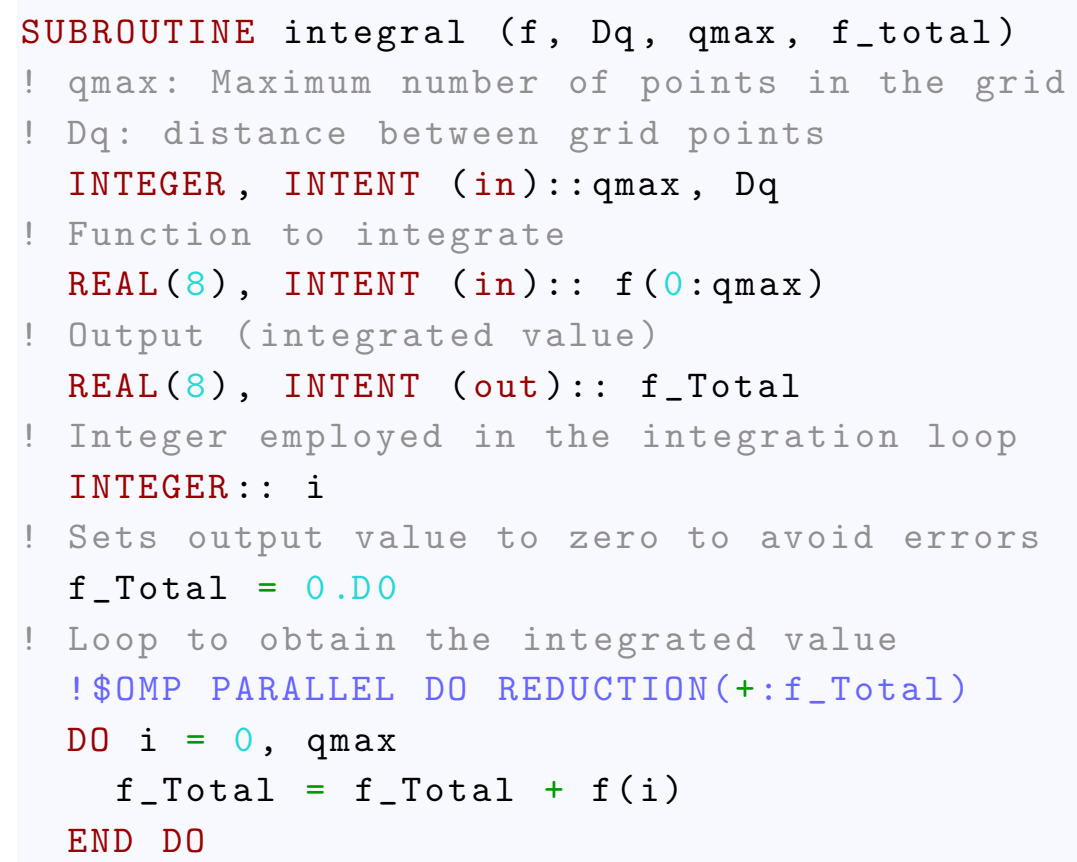




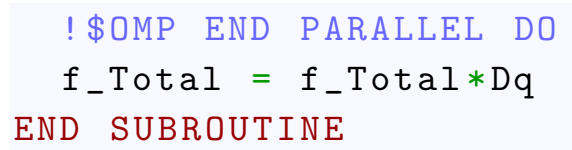

Listing B.2: Simple subroutine to compute the integral of $\mathrm{f}$ in a one-dimensional plannar domain equally spaced.

\section{B.3 Diffusion correction for conservation of energy}

In kinetic problems it is very important to compute the moments of the distribution function in an accurate way. Unfortunately, the general Gaussian propagator utilized to solve one and two-dimensional kinetic problems has a small deviation in the conservation of the second order moment of order $\sim \tau^{2}$. This is acceptable as we assume that the propagator is a first order approximation and $\tau \ll 1$, but we have found situations where this second condition is not completely fulfilled, which can create a numerical increment of the second order momentum.

To solve this, a high order propagator could be employed, but its calculation will be laborious and not always possible. A solution was first proposed in [41], which consist on a correction to the diffusive matrix to ensure the conservation of the three first order moments.

Basically, this correction consists in applying a modification to the diffusive parameter as

$$
D_{i j}^{*}=D_{i j}-\tau \frac{D_{i} D_{j}}{2}
$$

where $D_{i j}$ and $D_{i}$ are the diffusion and drift parameters in the direction $i$ and $j$, respectively. Here, $\tau$ is the time parameter and $D_{i j}^{*}$ is the final diffusion value passed to the propagator. An additional correction can still be used to improve the problem conservation even further. The reformulation of the stress tensor presented in equation (B.1) is redefined as

$$
D_{i j}^{*}=D_{i j}-\tau \alpha \frac{D_{i} D_{j}}{2},
$$

where $\alpha$ is a parameter that must be computed at each time step and it is order unity. Assuming for example that the total energy is conserved in a simulation, alpha can be defined as

$$
\alpha^{n+1}=\alpha^{n}+C\left(n E^{0}-n E^{n}\right),
$$

where superscript $n$ indicates the current iteration, $C$ is a constant of order $\tau-1$ and $n E$ is the energy of the system. This type of corrections results in an autocorrelation to ensure that the total energy of the problem is computed. However, situations may arise when the definition of this $\alpha^{n}$ requires the analysis of the drift-diffusive problem, as energy may not be always conserved and a rate of change must be assumed. 


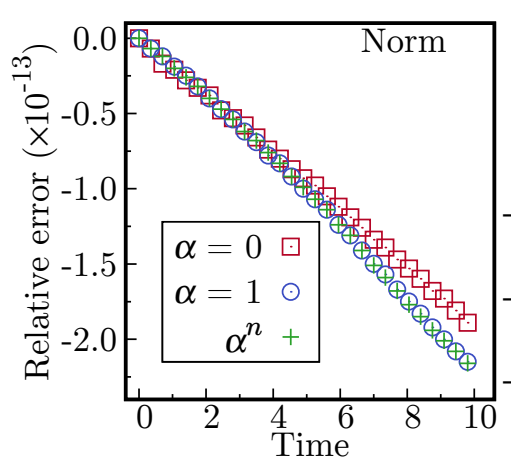

(a) Norm

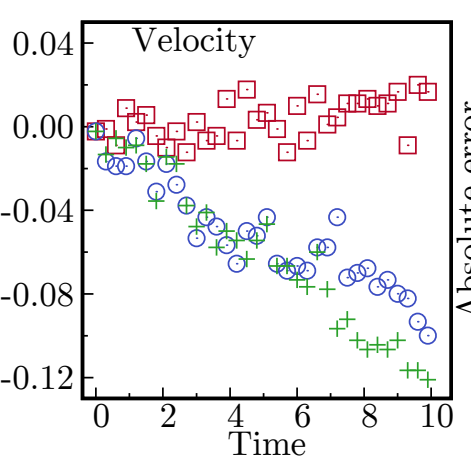

(b) Velocity

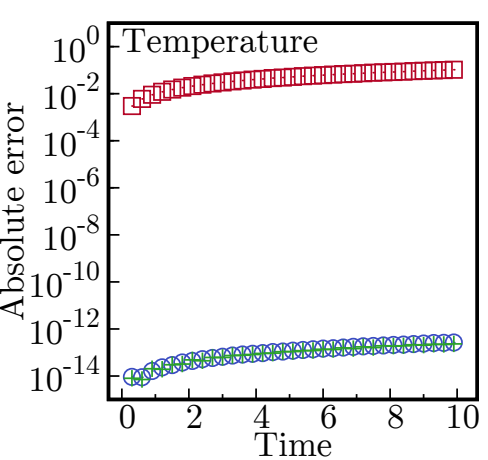

(c) Temperature

Figure B.2: $\quad$ Effect of the different corrections in the norm, velocity and temperature. $\alpha n$ represents the case where the autocorrelation function to conserve the total energy is used.

To test the different corrections, a simple one-dimensional case, based in the selfconsistent Dougherty collision operator, is presented. Three cases are compared: a case with no correction, equivalent to set $\alpha=0$; a case with the correction from equation (B.1), i.e., $\alpha=1$ and the case with the recalculation of $\alpha$ at each time step. Figure (B.2) depicts the errors respect to the initial condition of the norm, mean velocity and temperature. For norm and velocity, errors are small regardless the correction utilized, whereas temperature, figure B.2c has a huge increment if no correction of the diffusive parameter is used. Nevertheless, both corrections, the one with constant alpha and the one recalculated at each time step, have similar errors, in the order of $10^{-14}$.

In one-dimensional cases, where a large amount of points can be used, a correction like $\alpha=1$ is enough for all cases presented in this dissertation. On the other hand, twodimensional cases, as those studied in chapter 4 for the two velocity collision operators, require an autocorrelation, i.e., $\alpha$ variable, due to the low amount of points employed. Even with this corrections, errors in those cases are in the order of $10^{-6}$. This indicates that a more accurate correction is required in two velocity cases, which will be studied in future works.

\section{B.4 Adaptable mesh for two-dimensional purely convec- tive problems}

In section 2.3.2 it was presented how the 1D1V Vlasov problem requires an adaptation of the mesh to properly represent the solution. In this section, the subroutine employed to advance the grid is presented. The procedure proposed in this thesis is presented in listing B.3. It can be seen how the new grid $\mathrm{x} 2$, v2 is founded in the previous iterations coordinates $\mathrm{x}, \mathrm{v}$; the acceleration $\mathrm{Dv}$ and the velocity itself $\mathrm{v}$. The subroutine Insertion_Sort organize the new grid to ease the analysis, but it is not an obligatory process. This process is fast to compute, as seen in figure 2.25 , and does not require 
a large amount of computational resources. We remark here that the grid points are two-dimensional arrays, since each point dynamics is completely independent.

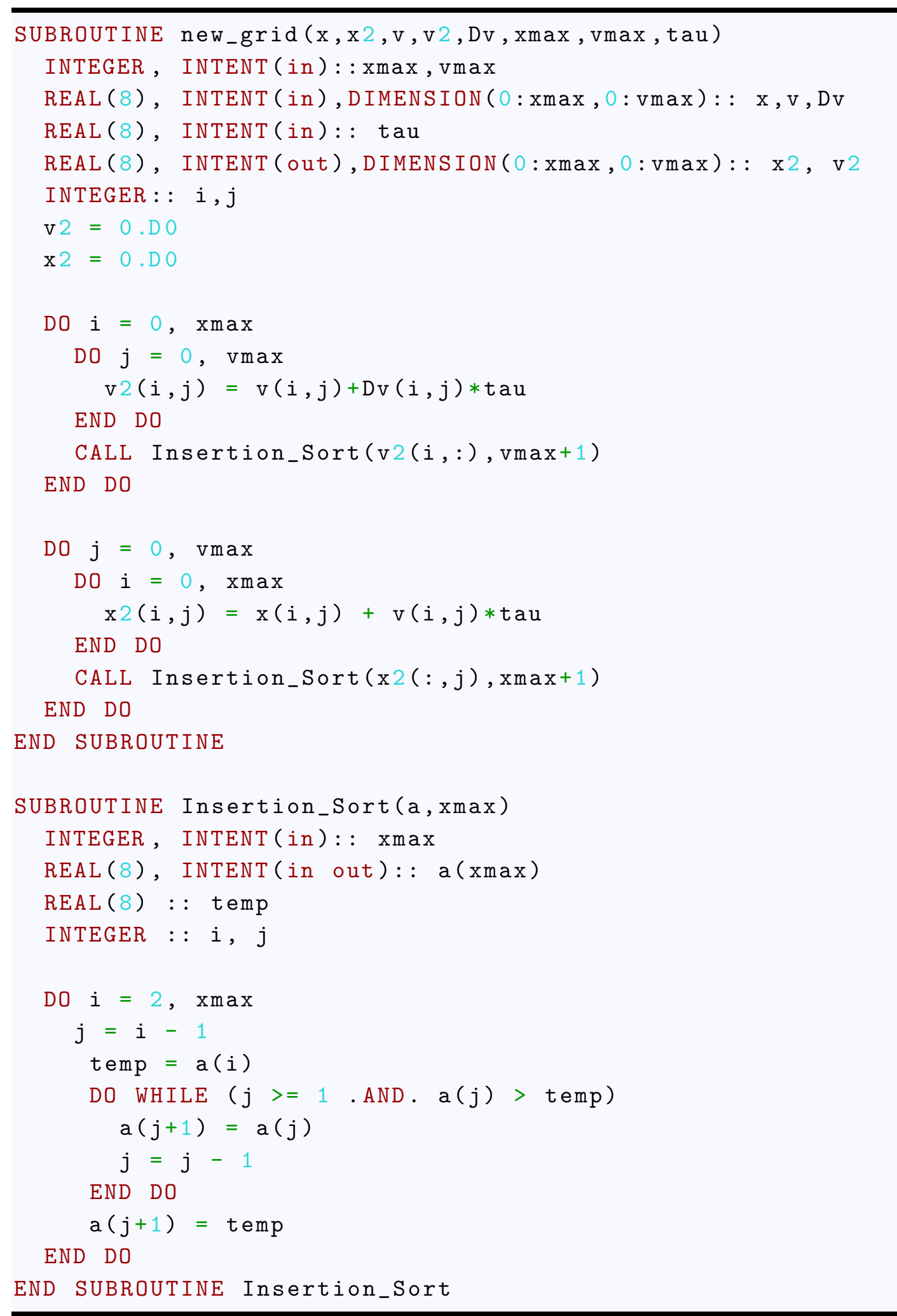

Listing B.3: Generation of the new grid for a $1 D 1 \mathrm{~V}$ Vlasov problem 


\section{B.5 Interpolation of a non-constant mesh}

As presented in section 2.3.2, grid motion required in two-dimensional purely convective problems requires an extra fixed mesh to allow the integration of the distribution function. In this work, we have employed a weighted interpolation algorithm to obtain this mesh-fixed function, which Fortran subroutine is presented in listing B.4. Nevertheless, other methods can be used to calculate the moments of $f$ without loosing generality and applicability of the PIM.

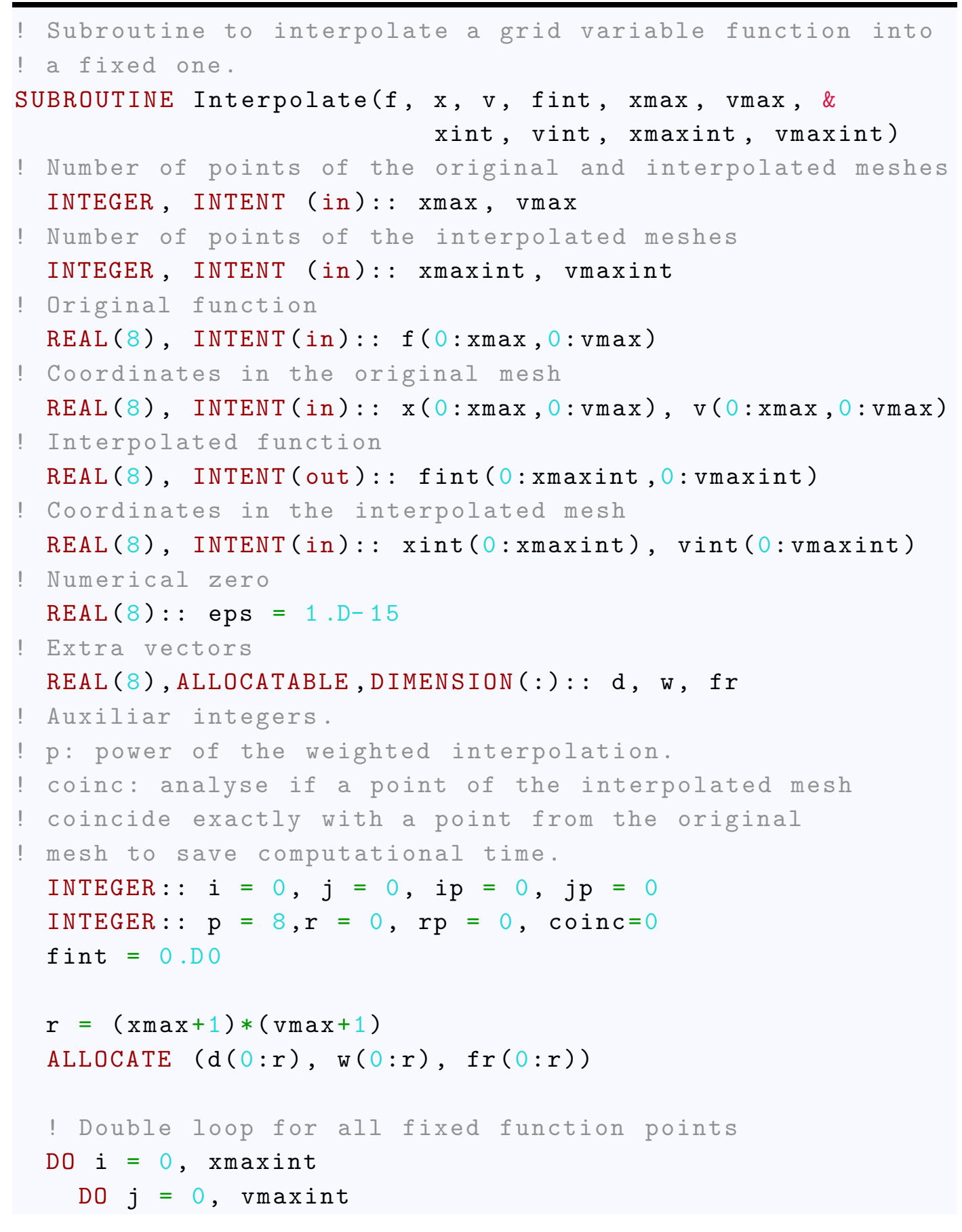




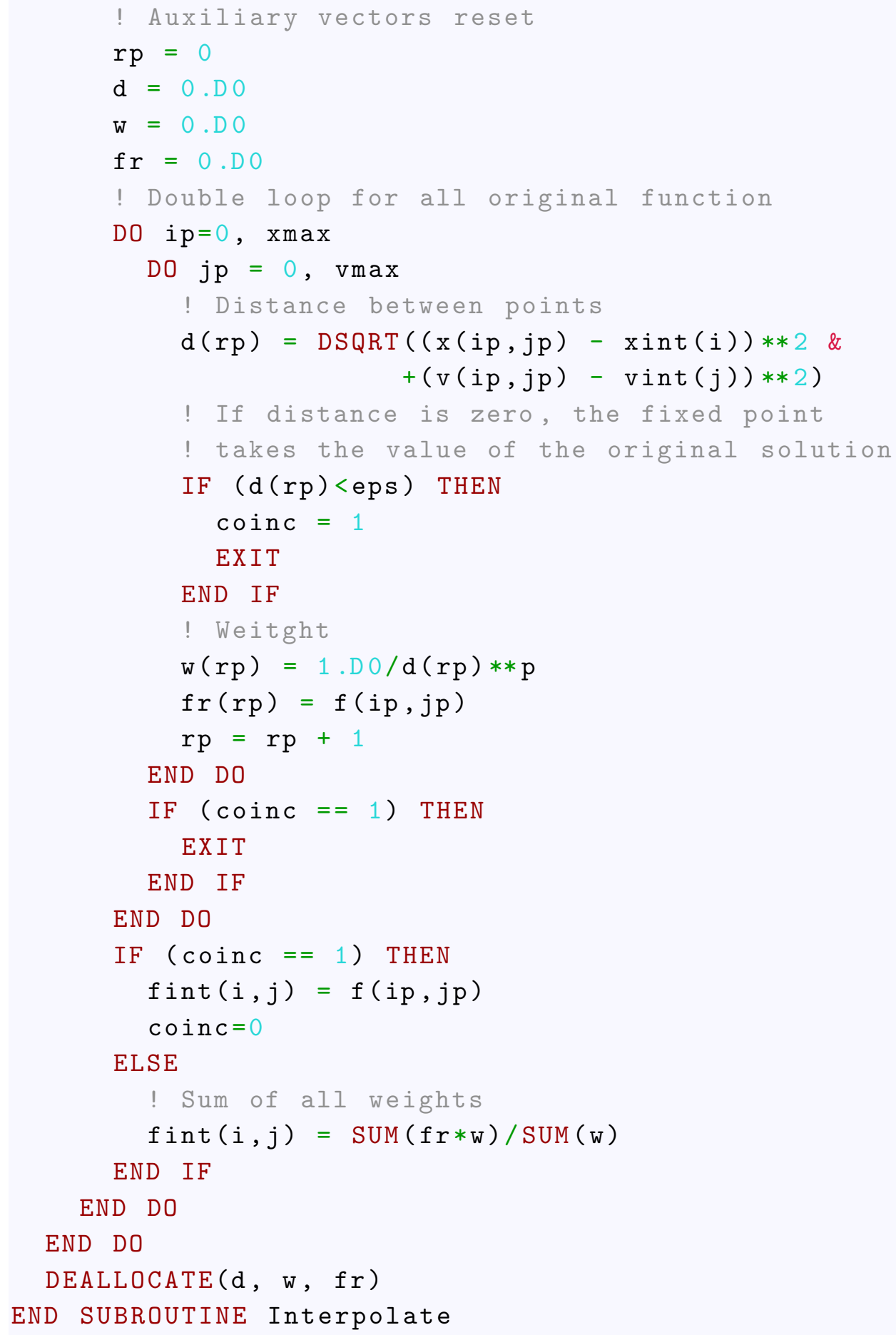

Listing B.4: Interpolation subroutine that provides an approximated solution in a fixed grid, where the calculation of macroscopic moments can be computed.

To test this subroutine, an example is presented now. The solution presented in figure 2.19 is interpolated for different values of $p=4,6,8$ at $t=0.5$ in figure B.3. It can be seen how the highest the parameter $p$, the closer the interpolation function is to the 


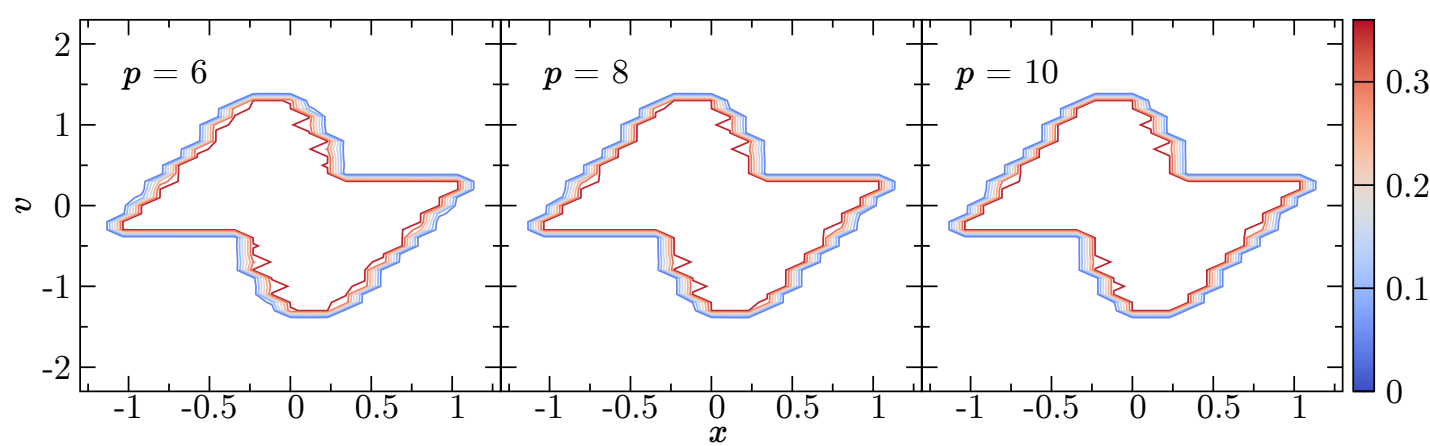

(a) $p=4$

(b) $p=6$

(c) $p=8$

Figure B.3: Interpolation of numerical solution for problem presented in figure 2.19 at $t=0.5$ for different values of $p$. The higher the points, the closer to the numerical unstructured solution the interpolated function is.

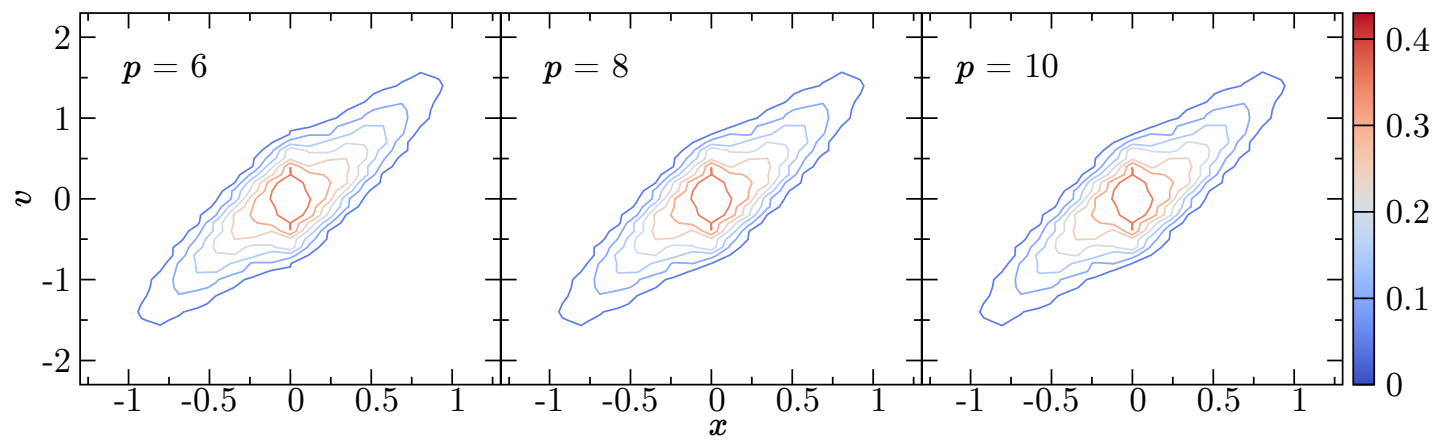

(a) $p=4$

(b) $p=6$

(c) $p=8$

Figure B.4: Interpolation of numerical solution for problem presented in figure 2.19 but with a double Gaussian initial function. Results are interpolated at at $t=0.5$ for different values of $p$.

abrupt original one. Nevertheless, a high value of $p$ produces not so good results with smooth functions, as can be seen in figure B.4, were the same example as in the previous case is presented, but with a double Gaussian initial condition. Here, the highest the parameter $p$, the more perturbed becomes the Gaussian function. This process is the more time consuming task performed in the resolution of purely convective problems, see figure 2.25 , and it should be improved to reduce the computational cost.

\section{B.6 Simple vs. double precision variables}

In this section, we compare solutions from this dissertation with double and simple precision variables. Through this work, double precision variables have been used in all codes. The aim of this comparison is to check if simple precision variables can be employed in the PIM without loosing accuracy, allowing to save space in RAM and computation time. 


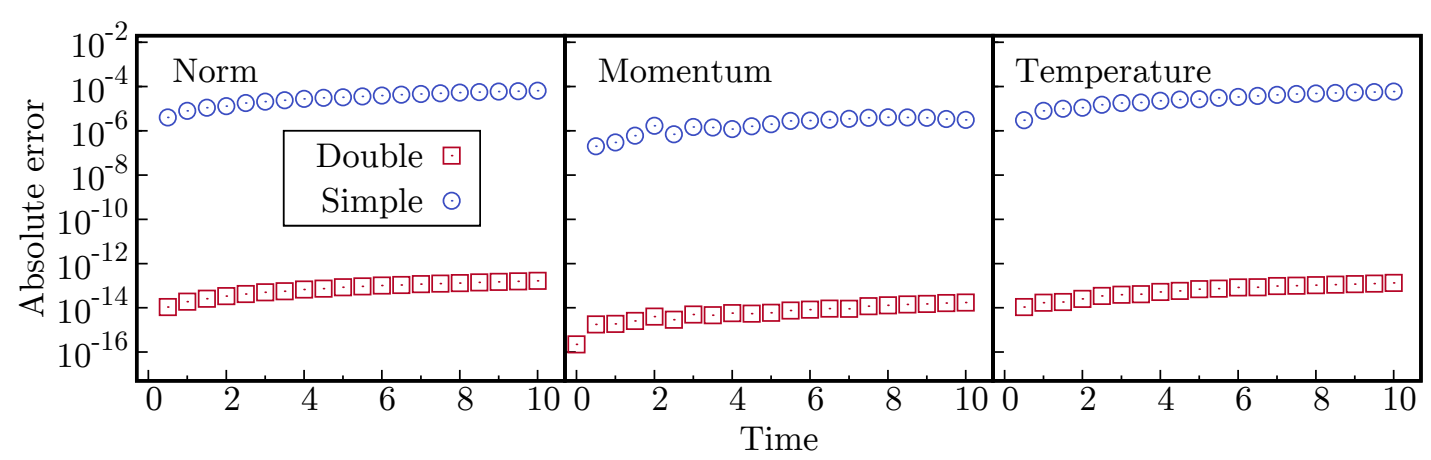

Figure B.5: Errors of the macroscopic quantities for the self-collisional one-dimensional Dougherty operator with double and simple precision.

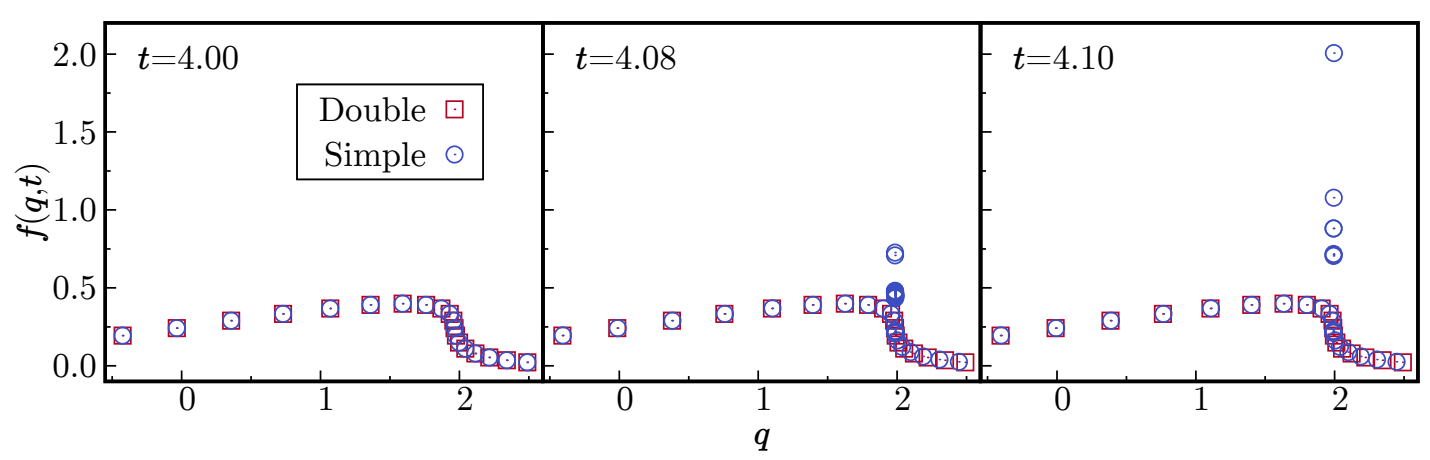

Figure B.6: Burgers' inviscid problem in double and simple precision. Simple precision presents issues where various points are closer, generating an increasing $f$.

Lets start by recovering the self-collision Dougherty numerical problem presented in section 4.1.4. With the same $\tau$ and grid, the double precision computed previously and a new case with simple precision variables are compared in figure B.5, where errors of macroscopic quantities for both cases are drawn. Due to the fewer decimals used in simple precision representation, higher errors respect to the initial conditions are found, which are big enough to affect the value in a simulation.

Lets analyse now a more involved problem where simple or double precision do not affect only the conservation properties but the evolution of the solution itself. When the pure convective propagator is employed, the mesh must be moved accorder to the convective force, as was explained in 2.3. For some non-linear values of $D_{q}$, the movement of the mesh may produce two points appearing really close to each other. Without enough precision, the numerical code may assume as coincident those points In figure B.6, the result for the inviscid Burgers' equation is presented for the cases of double and simple precision. It can be seen for the simple precision case, how some points highly increase its value, because the grid points are so close that it is impossible to difference them numerically, and are treated by the numerical integral scheme as the same point. Also, in this particular problem, the convective force is related with the value of $f$, which in this case will produce that the separated points feel a higher convective force. For this case, the precision of the computations not only affects the conservation of macroscopic 


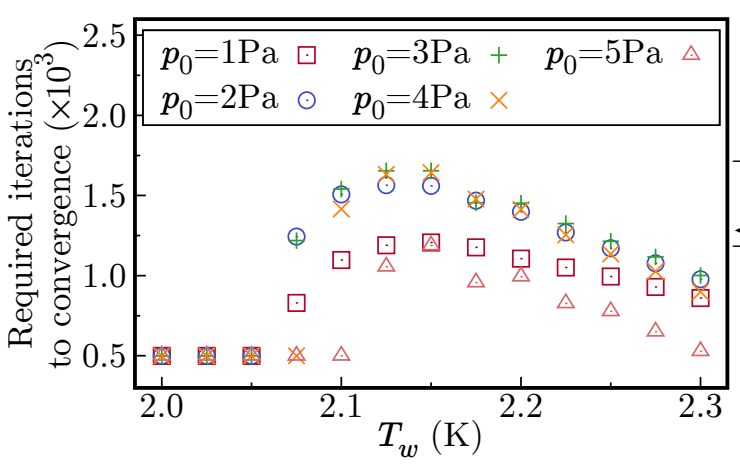

(a)

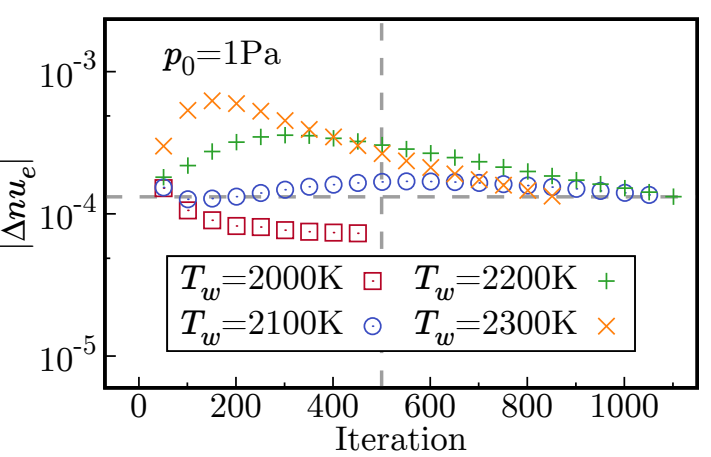

(b)

Figure B.7: Required number of iterations to reach convergence. (a) As a function of $T_{w}$ and $p_{0}$. (b) Evolution of $\left|\Delta n u_{e}\right|$ for $p_{0}=1 \mathrm{~Pa}$ and significant wall temperatures.

variables, but also the evolution of the problem itself. Hence, double precision variables must be utilized to ensure proper dynamics in all cases.

\section{B.7 Convergence criteria for plasma-wall discontinuous model}

To finish with the analysis of the numerical vicissitudes, let us recover the results presented in section 6.1.1 where the influence of the background pressure in the discontinuous plasma wall model was studied. For this model, a convergence criteria was defined to ensure that a quasi-state was reached for all range of wall temperatures and background pressures employed. Here, the evolution of this criteria is analysed from a numerical point of view. Figure B.7a shows the number of iterations required for each case of wall temperature and background pressure. For low temperatures the convergence criteria is fulfilled after 500 iterations, which is the minimum specified, whereas intermediate temperatures require more iterations, meaning that a complex interaction between the disparate phenomena in both zones arise, which hinders the evolution of the numerical simulation. Moreover, for high wall temperatures, a lower number of iterations is required. This hints that a stable situation is reached easily for high energy electrons as they relax faster. If the relative error between iterations $\left|\Delta n u_{e}\right|$ is analysed, figure B.7b, the complex system dynamics can be well detected. Low wall temperatures cases reach a stable condition faster, but as temperature increases, the simulations require more iterations to reach a quasi-steady state as a result of the complex interplay of disparate phenomena. For very high temperatures, the emission of high energy electrons dominates the system dynamics and the convergence criteria is rapidly fulfilled.

It is clear that a influence of the system properties in the electrons evolution arises, which influence not only the computational time required to complete the simulation but also the system dynamics. 



\section{LIST OF PUBLICATIONS AND CONFERENCES}

\section{Funding}

This work was partially funded by RFP ESA-IPL-PTM-PA-fg-2015-122-LE "Exploring angular-momentum phenomenology in aerothermodynamics and MHD".

\section{Contributions to referred publications}

1. J. Gonzalez, J.M. Donoso and L. Conde. Propagator Computational Method for Drift-Diffusion Equations to Describe Plasma-Wall Interaction. Contributions to Plasma Physics, Wiley Online Library, 2014, 54(3):298

2. J. Gonzalez, J.M. Donoso and S. Tierno. Three species one-dimensional kinetic model for weakly ionized plasmas. Physics of Plasmas, 2016, 23(6):062311

3. J. Gonzalez, S. Tierno and J.M. Donoso. Comparison between experimental Langmuir probes and three species one-dimensional kinetic simulations. Physics of Plasmas, 2016, 23(10):103514

4. J.M. Donoso, A. Jimenez, J. Gonzalez and L. Conde. Integral propagator method as a kinetic operator to describe discontinuous plasmas. Journal of Physics: Conference Series, 2016, 768(1):012004.

5. J.M. Donoso, J. Gonzalez. Boundary-path-sum method for drift-diffusion equations in constrained domains. Submitted 
6. J. Gonzalez, J.M. Donoso. Effect of the ionization ratio in a three species one-dimensional kinetic model. Submitted

7. J. Gonzalez. Implementation of collisional effects in particle-in-cell methods using short-time probability transition functions. In preparation

8. J.M. Donoso, J. Gonzalez, J. L. Domenech-Garret. Thermionic emission into a vacuum chamber described by a discontinuous wall-plasma kinetic model. In preparation

9. J. Gonzalez, J.M. Donoso, L. Conde. Kinetic description to study the evolution of ions in the plume of a gridded thruster. In preparation

10. J.M. Donoso, J. Gonzalez, P. Solano-López. Propagator integral method for non-diffusive equations. In preparation

\section{Contributions to conferences}

1. J.M. Donoso, A. Jiménez , J. Gonzalez, A. González and L. Conde. Propagator method as a kinetic operator to analyse discontinuities in plasmas. $6^{\text {th }}$ International Workshop \& Summer School on Plasma Physics (IWSSPP), Kiten, Bulgaria, 2014. Invited lecture.

2. L. Conde, J.L. Domenech-Garret, S.P. Tierno, E. Del Río, J. Gonzalez, P. Solano-López, P. Argente and J.M. Donoso. The Plasma Testing Facility at the Technical University of Madrid (UPM). Electric Propulsion Innovation 8 Competitiveness Workshop, Brussels, Belgium, 2014. Oral presentation.

3. L. Conde, J.L. Domenech-Garret, E. Del Río, J. Gonzalez, P. Solano-López, P. Argente and J.M. Donoso. Development status of ALPHIE. Electric Propulsion Innovation \& Competitiveness Workshop, Brussels, Belgium, 2014. Oral presentation.

4. J.M. Donoso, J. Gonzalez and P. Solano-López. Numerical Integral Method for kinetic equations in gases and plasmas. Proceedings of the 8th European Symposium on Aerothermodynamics for Space Vehicles, Lisbon, Portugal, 2015. Oral presentation.

5. J. Gonzalez, S.P. Tierno and J.M. Donoso. Kinetic description to analyse collecting probe measurements in weakly ionized plasmas. $11^{\text {th }}$ International Workshop on Electric Probes in Magnetized Plasmas (IWEP), Sozopol, Bulgaria, 2015. Oral presentation.

6. D. Giordano, J. Gonzalez and J.M. Donoso. Perfect-gas thermodynamic model including angular momentum as state parameter. $14^{\text {th }}$ Joint European Thermodynamics Conference, Budapest, Hungary, 2017. Oral presentation. 


\section{Seminars}

1. J. Gonzalez, J.M. Donoso. Propagator Integral Method for Plasma Physics kinetic equations with practical applications. Deutsches Zentrum für Luft- und Raumfahrt e.V., Göttingen, Germany, 2016

\section{Master thesis}

1. A. Gonzalez. Modelización numérica con esquema PIM del plasma en un motor iónico. Advisor: J.M. Donoso, Co-advisor: J. Gonzalez, 2016 



\section{BIBLIOGRAPHY}

[1] I. Langmuir. Oscillations in ionized gases. Proceedings of the National Academy of Sciences, 14(8):627-637, 1928.

[2] L. Tonks and I. Langmuir. Oscillations in Ionized Gases. Phys. Rev. D, 33:195210, Feb 1929.

[3] J. van Dijk. Modelling of plasma light sources: an object-oriented approach. $\mathrm{PhD}$ thesis, Technische Universiteit Eindhoven, 2001.

[4] JET Team et al. Fusion energy production from a deuterium-tritium plasma in the JET tokamak. Nucl. Fusion, 32(2):187, 1992.

[5] G. A Collins, R. Hutchings, K. T. Short, J. Tendys, and C. H. Van Der Valk. Development of a plasma immersion ion implanter for the surface treatment of metal components. Surf. Coat. Technol., 84(1-3):537-543, 1996.

[6] M. C. Kim, S. H. Yang, J. H. Boo, and J. G. Han. Surface treatment of metals using an atmospheric pressure plasma jet and their surface characteristics. Surf. Coat. Technol., 174:839-844, 2003.

[7] J. E. Foster. Plasma-based water purification: Challenges and prospects for the future. Phys. Plasmas, 24(5):055501, 2017.

[8] D. M. Goebel and I. Katz. Fundamentals of electric propulsion: ion and Hall thrusters, volume 1. John Wiley \& Sons, 2008.

[9] H. C. Yee and B. Sjögreen. Development of low dissipative high order filter schemes for multiscale Navier-Stokes/MHD systems. J. Comput. Phys., 225(1):910-934, 2007.

[10] X. Zhao and C. E. Seyler. Computational extended magneto-hydrodynamical study of shock structure generated by flows past an obstacle. Phys. Plasmas, 22(7):-, 2015. 
[11] T. D. Arber and R. G. L. Vann. A critical comparison of Eulerian-grid-based Vlasov solvers. J. Comput. Phys., 180(1):339-357, 2002.

[12] M. Sengupta and R. Ganesh. Influence of electron-neutral elastic collisions on the instability of an ion-contaminated cylindrical electron cloud: 2D3V PIC-withMCC simulations. Phys. Plasmas, 23(10), 2016.

[13] S. Cho, H. Watanabe, K. Kubota, S. Iihara, K. Fuchigami, K. Uematsu, and I. Funaki. Study of electron transport in a Hall thruster by axial-radial fully kinetic particle simulation. Phys. Plasmas, 22(10):103523, 2015.

[14] H. C. Kim, Felipe I., S. S. Yang, M. Radmilović-Radjenović, and J. K. Lee. Particle and fluid simulations of low-temperature plasma discharges: benchmarks and kinetic effects. J. Phys. D, 38(19):R283, 2005.

[15] V. Kolobov and R. Arslanbekov. Electrostatic PIC with adaptive Cartesian mesh. JPCS, 719(1):012020, 2016.

[16] W. M. Manheimer, M. Lampe, and G. Joyce. Langevin representation of Coulomb collisions in PIC simulations. J. Comput. Phys., 138(2):563-584, 1997.

[17] V. Vahedi and M. Surendra. A Monte Carlo collision model for the particle-in-cell method: applications to argon and oxygen discharges. Comput. Phys. Comm., 87(1):179 - 198, 1995.

[18] O. Pezzi, F. Valentini, and P. Veltri. Collisional Relaxation of Fine Velocity Structures in Plasmas. Phys. Rev. Lett., 116(14):145001, 2016.

[19] O. Pezzi, F. Valentini, and P. Veltri. Collisional relaxation: Landau versus Dougherty operator. J. Plasma Phys., 2015.

[20] E. Hirvijoki. Action principle for Coulomb collisions in plasmas. Phys. Plasmas, 23(9):094502, 2016.

[21] J. Gonzalez. Design of a Semi-analytical Method to Describe Plasma-Wall Interactions. Master's thesis, Escuela Técnica Superior de Ingenieros Aeronáuticos, September 2013.

[22] J. M. Donoso, J. J. Salgado, and M. Soler. Short-time propagators for nonlinear Fokker-Planck equations. J. Phys. A: Math. Theor., 32(20):3681, 1999.

[23] J. Gonzalez, J. M. Donoso, and L. Conde. Propagator Computational Method for Drift-Diffusion equations to Describe Plasma-Wall Interaction. Contrib. Plasma Phys., 54(3):298-303, 2014.

[24] J. M. Donoso, J. Gonzalez, and P. Solano-López. Numerical integral method for kinetic equations in gases and plasmas. In Proceedings of the 8th European Symposium on Aerothermodynamics for Space Vehicles. European Space Agency, 2015. 
[25] J. Gonzalez, J. M. Donoso, and S. P. Tierno. Three species one-dimensional kinetic model for weakly ionized plasmas. Phys. Plasmas, 23(6), 2016.

[26] J. M. Donoso, A. Jimenez, J. Gonzalez, and L. Conde. Integral propagator method as a kinetic operator to describe discontinuous plasmas. JPCS, 768(1):012004, 2016.

[27] J. M. Donoso and J. Gonzalez. Boundary-path-sum method for Drift-Diffusion equations in constrained domains. Under Review, January 2017.

[28] J. Gonzalez, S. P. Tierno, and J. M. Donoso. Comparison between experimental Langmuir probes and three species one-dimensional kinetic simulations. Phys. Plasmas, 23(10):103514, 2016.

[29] W. X. Xie, W. X., and L. Cai. Numerical meshfree path integration method for non-linear dynamic systems. Appl. Math. Comput., 197(1):426-434, 2008.

[30] D. Janakiraman and K. L. Sebastian. Path Integral Formulation for Lévy flights: Evaluation of the propagator for free, linear, and harmonic potentials in the overand underdamped limits. Phys. Rev. E, 86(6):061105, 2012.

[31] F. Mota-Furtado and P. F. O'Mahony. Exact propagator for generalized OrnsteinUhlenbeck processes. Phys. Rev. E, 75(4):041102, 2007.

[32] C. Wichaidit and W. N. G. Hitchon. Propagator methods for plasma simulations: application to breakdown. J. Comput. Phys., 203(2):650-667, 2005.

[33] M. F. Wehner and W. G. Wolfer. Numerical evaluation of path-integral solutions to Fokker - Planck equations. III. Time and functionally dependent coefficients. Phys. Rev. A, 35(4):1795, 1987.

[34] A. Navarra, J. Tribbia, and G. Conti. The path integral formulation of climate dynamics. PloS one, 8(6):e67022, 2013.

[35] T. Kosztolowicz. Phase-space diffusion in a system with a partially permeable wall. Journal of Physics A: Mathematical and General, 31(8):1943, 1998.

[36] A. Carpio and G. Duro. Well posedness of an integrodifferential kinetic model of Fokker-Planck type for angiogenesis. Nonlinear Anal. Real World Appl., 30:184-212, 2016.

[37] H. T. Zhu and L. L. Duan. Probabilistic solution of non-linear random ship roll motion by path integration. Int. J. Non-Linear Mech., 83:1-8, 2016.

[38] G. Tsedendorj and H. Isshiki. Numerical Solution of Two-Dimensional AdvectionDiffusion Equation Using Generalized Integral Representation Method. Int. J. Comput. Meth., 0(0):1750028, 2016.

[39] P. Kumar and S. Narayanan. Efficient path integral solution of Fokker-Planck equation: response, bifurcation and periodicity of nonlinear systems. Int. J. Adv. Eng. Sci. Appl. Math., 3(1-4):111-125, 2011. 
[40] L. Cai, Y. Nie, W. Xie, and W. Zhang. Numerical path integration method based on bubble grids for nonlinear dynamical systems. Appl. Math. Model., 37(3):1490-1501, 2013.

[41] J. M. Donoso. Métodos numéricos integrales para ecuaciones Fokker-Planck. PhD thesis, Universidad Complutense de Madrid, 1995.

[42] J. M. Donoso, J. J. Salgado, and M. Soler. Non-linear Fokker-Planck integral propagator for plasma kinetic coefficients. J. Plasma Phys., 38(41):9145, 2005.

[43] J. M. Donoso and J. J. Salgado. Nonlinear Fokker-Planck-Landau integral propagator (II): transport far from equilibrium. J. Plasma Phys., 39(40):12587, 2006.

[44] J. M. Donoso and E. del Rio. Integral propagator solvers for Vlasov-FokkerPlanck equations. J. Plasma Phys., 40(24):F449, 2007.

[45] S. Chandrasekhar. Stochastic problems in physics and astronomy. Rev. Mod. Phys., 15:1-89, Jan 1943.

[46] G. Barton. Elements of Green's functions and propagation: potentials, diffusion, and waves. Oxford University Press, 1989.

[47] A. D. Polyanin. Handbook of linear partial differential equations for engineers and scientists. CRC press, 2010.

[48] D. G. Duffy. Green's functions with applications. CRC Press, 2010.

[49] K. D. Cole, J. V. Beck, A. Haji-Sheikh, and B. Litkouhi. Heat conduction using Green's functions. Taylor \& Francis, 2010.

[50] Y. L. Chang, Y. T. Lee, L. J. Jiang, and J. T. Chen. Green's Function Problem of Laplace Equation with Spherical and Prolate Spheroidal Boundaries by Using the Null-Field Boundary Integral Equation. Int. J. Comput. Meth., 0(0):1650020, 0 .

[51] A. Naess and V. Moe. Efficient path integration methods for nonlinear dynamic systems. Probabilistic Eng. Mech., 15(2):221-231, 2000.

[52] M. F. Wehner and W. G. Wolfer. Numerical evaluation of path-integral solutions to Fokker-Planck equations. II. Restricted stochastic processes. Phys. Rev. A, 28(5):3003, 1983.

[53] P. Szymczak and A. J. C. Ladd. Boundary conditions for stochastic solutions of the convection-diffusion equation. Phys. Rev. E, 68(3):036704, 2003.

[54] S. V. G. Menon, V. Kumar, and D. C. Sahni. Green's function approach to the solution of the time dependent Fokker-Planck equation with an absorbing boundary. Phys. A, 135(1):63-79, 1986. 
[55] M. A. Burschka and U. M. Titulaer. The kinetic boundary layer for the FokkerPlanck equation with absorbing boundary. J. Stat. Phys., 25(3):569-582, 1981.

[56] U. S Inan and M. Gołkowski. Principles of plasma physics for engineers and scientists. Cambridge University Press, 2010.

[57] M. Keidar and I. Beilis. Plasma engineering: Applications from aerospace to bio and nanotechnology. Academic Press, 2013.

[58] T. D. Frank. Nonlinear Fokker-Planck equations: fundamentals and applications. Springer Science \& Business Media, 2005.

[59] H. Risken. Fokker-Planck Equation. Springer, 1984.

[60] H. S. Carslaw. Introduction to the mathematical theory of the conduction of heat in solids. Lon don, 1921.

[61] H. Touchette, E. Van der Straeten, and W. Just. Brownian motion with dry friction: Fokker-Planck approach. J. Plasma Phys., 43(44):445002, 2010.

[62] A. Araújo, A. K. Das, and E. Sousa. A numerical approach to study the Kramers equation for finite geometries: boundary conditions and potential fields. J. Phys. A, 48(4):045202, 2015.

[63] J. I. Jiménez-Aquino and M. Romero-Bastida. Fokker-Planck-Kramers equation for a Brownian gas in a magnetic field. Phys. Rev. E, 74(4):041117, 2006.

[64] H. A. Kramers. Brownian motion in a field of force and the diffusion model of chemical reactions. Phys. A, 7(4):284-304, 1940.

[65] A. B. de Monvel-Berthier and P. Dita. Brownian motion near an absorbing sphere. J. Stat. Phys., 62(3):729-736, 1991.

[66] Y. S. Mayya. Brownian motion near a plane absorber: Nonanalyticity of the boundary layer solutions to the Fokker-Planck equation. J. Chem. Phys, 82(4):2033-2039, 1985.

[67] M. A. Burschka and U. M. Titulaer. The kinetic boundary layer for the FokkerPlanck equation: A Brownian particle in a uniform field. Phys. A, 112(1):315-330, 1982 .

[68] C. W. Gardiner. Stochastic methods. Springer-Verlag, Berlin-Heidelberg-New York-Tokyo, 1985.

[69] K. Rohlena and H. R. Skullerud. Transport coefficients in a weakly ionized nonequilibrium plasma. Phys. Rev. E, 51(6):6028, 1995.

[70] E. V. Kustova and E. A. Nagnibeda. Transport properties of a reacting gas mixture with strong vibrational and chemical nonequilibrium. Chemical Physics, 233(1):57 - 75, 1998. 
[71] S. Longo and A. Milella. A one-dimensional, self-consistent model of charged particle transport and vibrational kinetics in weakly ionized hydrogen. Chem. Phys., 274(2):219-229, 2001.

[72] E. Nagnibeda and E. V. Kustova. Non-equilibrium reacting gas flows: kinetic theory of transport and relaxation processes. Springer Science \& Business Media, 2009.

[73] V. A. Istomin and E. V. Kustova. State-specific transport properties of partially ionized flows of electronically excited atomic gases. Chemical Physics, 485-486:125 - 139, 2017.

[74] V. A. Istomin and E. V. Kustova. Transport coefficients and heat fluxes in non-equilibrium high-temperature flows with electronic excitation. Physics of Plasmas, 24(2):022109, 2017.

[75] R. G. Keanini. Green's function-stochastic methods framework for probing nonlinear evolution problems: Burger's equation, the nonlinear Schrödinger's equation, and hydrodynamic organization of near-molecular-scale vorticity. Ann. Phys., 326:1002-1031, April 2011.

[76] Y. C. Hon and X. Z. Mao. An efficient numerical scheme for Burgers' equation. Applied Mathematics and Computation, 95(1):37-50, 1998.

[77] E. Medina, T. Hwa, M. Kardar, and Y. C. Zhang. Burgers equation with correlated noise: Renormalization-group analysis and applications to directed polymers and interface growth. Physical Review A, 39(6):3053, 1989.

[78] J. A. Bittencourt. Fundamentals of Plasma Physics. Springer Science \& Business Media, 2013.

[79] T. J. M. Boyd and J. J. Sanderson. The physics of plasmas. Cambridge University Press, 2003.

[80] P. L. Bhatnagar, E. P. Gross, and M. Krook. A model for collision processes in gases. I. Small amplitude processes in charged and neutral one-component systems. Phys. Rev. D, 94(3):511, 1954.

[81] E. P. Gross and M. Krook. Model for collision processes in gases: Small-amplitude oscillations of charged two-component systems. Phys. Rev. D, 102(3):593, 1956.

[82] B. A. Trubnikov. Particle Interactions in a Fully Ionized Plasma. Rev. Plasma Phys., 1:105, 1965.

[83] S. P. Tierno, E. Roibás, J. L. Domenech-Garret, J. M. Donoso, and L. Conde. Measurements of Plasma Properties using the Floating Potential of Emissive Langmuir Probes. Contrib. Plasma Phys., 53(1):16-21, 2013.

[84] J. P. Sheehan and N. Hershkowitz. Emissive probes. Plasma Sources Sci. Technol., 20(6):063001, 2011. 
[85] T. Gyergyek and J. Kovačič. Saturation of a floating potential of an electron emitting electrode with increased electron emission: A one-dimensional kinetic model and particle-in-cell simulation. Phys. Plasmas, 19(1):013506, 2012.

[86] V. Pierrard and M. Lazar. Kappa distributions: theory and applications in space plasmas. Solar Phys., 267(1):153-174, 2010.

[87] M. P. Leubner. Fundamental issues on kappa-distributions in space plasmas and interplanetary proton distributions. Phys. Plasmas, 11(4):1308-1316, 2004.

[88] J. L. Domenech-Garret, S. P. Tierno, and L. Conde. Non-equilibrium thermionic electron emission for metals at high temperatures. J. of Appl. Phys., 118(7):074904, 2015.

[89] M. D. Campanell and M. V. Umansky. Are two plasma equilibrium states possible when the emission coefficient exceeds unity? Phys. Plasmas, 24(5):057101, 2017.

[90] P. M. Bellan. Fundamentals of Plasma Physics. Cambridge University Press, 2008.

[91] J. P. Dougherty, S. R. Watson, and M. A. Hellberg. Model Fokker-Planck Equations: Part 3. Application to transport phenomena. J. Plasma Phys., 1(03):327339, 1967.

[92] I. M. Tkachenko and P. Fernández de Córdoba. Transport properties of strongly coupled plasmas. Phys. Rev. E, 57:2222-2229, Feb 1998.

[93] J. R. Saraf. Transport processes in two component plasma on the basis of model Fokker-Planck equations. In Proceedings of the Indian Division of the Institution of Electronic and Radio Engineers, volume 6, pages 171-176. IET, 1968.

[94] O. Pezzi, F. Valentini, and P. Veltri. Nonlinear regime of electrostatic waves propagation in presence of electron-electron collisions. Physics of Plasmas (1994present), 22(4):042112, 2015.

[95] I. L. Semenov, A. G. Zagorodny, and I. V. Krivtsun. On the effect of ion-neutral collisions on dust grain screening in a low-pressure gas discharge plasma. Phys. Plasmas, 19(4):043703, 2012.

[96] D. D. Ryutov. Landau damping: half a century with the great discovery. Plasma Phys. Control. Fusion, 41(3A):A1, 1999.

[97] L. Oksuz and N. Hershkowitz. Plasma, presheath, collisional sheath and collisionless sheath potential profiles in weakly ionized, weakly collisional plasma. Plasma Sources Sci. Technol., 14(1):201, 2005.

[98] J. P. Sheehan, I. D. Kaganovich, H. Wang, D. Sydorenko, Y. Raitses, and N. Hershkowitz. Effects of emitted electron temperature on the plasma sheath. Phys. Plasmas, 21(6):063502, 2014. 
[99] E. Criado, E. Roibás, S. P. Tierno, P. Rodríguez De Francisco, J. L. DomenechGarret, J. M. Donoso, and L. Conde. Ion beam neutralization and properties of plasmas from low power ring cusp ion thrusters. Phys. Plasmas, 19(2):023505, 2012.

[100] E. Roibás, S. P. Tierno, E. Criado, J. L. Domenech-Garret, J. M. Donoso, and L. Conde. Characterization of the Ion Beam Neutralization of Plasma Thrusters Using Collecting and Emissive Langmuir Probes. Contrib. Plasma Phys., 53(1):57-62, 2013.

[101] H. Xu, Z. M. Sheng, X. M. Kong, and F. F. Su. Effects of ion motion on linear Landau damping. Phys. Plasmas, 24(2):022101, 2017.

[102] E. V . Stenson, J. Horn-Stanja, M. R. Stoneking, and T. S. Pedersen. Debye length and plasma skin depth: two length scales of interest in the creation and diagnosis of laboratory pair plasmas. J. Plasma Phys., 83(1), 2017.

[103] A. V. Ivanov, Iver H. Cairns, and P. A. Robinson. Wave damping as a critical phenomenon. Phys. Plasmas, 11(10):4649-4661, 2004.

[104] F. L. Hinton. Collisional transport in plasma. Handbook of Plasma Physics, 1:147, 1983.

[105] B. M. Smirnov. Physics of ionized gases. John Wiley \& Sons, 2008.

[106] P. P. Schram. Kinetic theory of gases and plasmas. Springer Science \& Business Media, 1991.

[107] J. Sykes. Short-time kinetic equations for hard spheres: Comparison with other theories. Journal of Statistical Physics, 8(3):279-292, 1973.

[108] S. Y. Ha. L 1 stability of the Boltzmann equation for the hard-sphere model. Arch. Rational Mech. Anal., 173(2):279-296, 2004.

[109] M. W. Anderson and T. M. O'Neil. Eigenfunctions and eigenvalues of the Dougherty collision operator. Phys. Plasmas, 14(5):052103, 2007.

[110] J. P. Dougherty. Model Fokker-Planck equation for a plasma and its solution. Phys. Fluids, 7(11):1788-1799, 1964.

[111] J. P. Dougherty and S. R. Watson. Model Fokker-Planck Equations: Part 2. The equation for a multicomponent plasma. J. Plasma Phys., 1(03):317-326, 1967.

[112] J. Gonzalez and J. M. Donoso. Effect of the ionization ratio in a three species one-dimensional kinetic model. Under Review, 2017.

[113] J. L. Domenech-Garret, S. P. Tierno, and L. Conde. Enhanced thermionic currents by non equilibrium electron populations of metals. EPJ B, 86(9):1-4, 2013. 
[114] L. A. Schwager. Effects of secondary and thermionic electron emission on the collector and source sheaths of a finite ion temperature plasma using kinetic theory and numerical simulation. Phys. Fluids B, 5(2):631-645, 1993.

[115] C. K. Goertz and G. Joyce. Numerical simulation of the plasma double layer. Astrophysics and space science, 32(1):165-173, 1975.

[116] T. Gyergyek, B. Jurčič-Zlobec, M. Čerček, and J. Kovačič. Sheath structure in front of an electron emitting electrode immersed in a two-electron temperature plasma: a fluid model and numerical solutions of the Poisson equation. Plasma Sources Sci. Technol., 18(3):035001, 2009.

[117] S. F. Masoudi. The effects of ion-neutral collision frequency on the plasma sheath dynamics for oblique entrance of ions into the sheath. EPJ D, 64(2):369-373, 2011.

[118] C. Böhm and J. Perrin. Retarding-field analyzer for measurements of ion energy distributions and secondary electron emission coefficients in low-pressure radio frequency discharges. Rev. Sci. Instrum., 64(1):31-44, 1993.

[119] S. A. Maiorov, O. F. Petrov, and V. E. Fortov. Calculation of resonant charge exchange cross sections of ions of rubidium, cesium, mercury, and noble gases. In Proceedings of the 34th EPS Conference on Plasma Physics, Warsaw, 2007.

[120] K. U. Riemann. Kinetic theory of the plasma sheath transition in a weakly ionized plasma. Phys. Fluids, 24(12):2163-2172, 1981.

[121] M. Lampe, T. B. Röcker, G. Joyce, S. K. Zhdanov, A. V. Ivlev, and G. E. Morfill. Ion distribution function in a plasma with uniform electric field. Phys. Plasmas, 19(11):113703, 2012.

[122] K. Kumar, H. R. Skullerud, and R. E. Robson. Kinetic theory of charged particle swarms in neutral gases. Aust. J. Phys., 33(2):343-448, 1980.

[123] I. Choquet, P. Degond, and B. Lucquin-Desreux. A hierarchy of diffusion models for partially ionized plasmas. Discrete Contin. Dyn. Syst. Ser. B, 8(4):735-772, 2007.

[124] I. Choquet, P. Degond, and B. Lucquin-Desreux. A strong ionization model in plasma physics. Math. Comput. Model, 49(1):88-113, 2009.

[125] I. Choquet and B. Lucquin-Desreux. Hydrodynamic limit for an arc discharge at atmospheric pressure. J. Stat. Phys., 119(1-2):197-239, 2005.

[126] E. Marsch. Kinetic physics of the solar wind plasma. In Physics of the Inner Heliosphere II, pages 45-133. Springer, 1991.

[127] L. Conde, J. L. Domenech-Garret, , J. M. Donoso, J. Damba, S. P. Tierno, E. Alamillo-Gamboa, and M. A. Castillo. Supersonic plasma beams with controlled speed generated by the alternative low power hybrid ion engine (ALPHIE) 
for space propulsion. Submitted for its publication to Plasma Source. Sci. and Technol., May 2017.

[128] G. Pocobelli. Charged-neutral collision models in the presence of drifts. Phys. Plasmas, 3(11):3975-3982, 1996.

[129] J. D. Huba. NRL: plasma formulary. Technical report, DTIC Document, 2009.

[130] M. Mitchner and C. H. Kruger. Partially ionized gases, volume 8. Wiley New York, 1973.

[131] R. L. Merlino. Understanding Langmuir probe current-voltage characteristics. Am. J. Phys., 75(12):1078-1085, 2007.

[132] F. F. Chen. Plasma Diagnostics Techniques, chapter 4. Electric probes, page 113. Academic Press, 1965.

[133] N. Hershkowitz. Plasma Diagnostics: Discharge Parameters and Chemistry, volume 1, chapter 3. How Langmuir Probes Work, page 113. Academic Press, 1989.

[134] V. I. Demidov, S. V. Ratynskaia, and K. Rypdal. Electric probes for plasmas: The link between theory and instrument. Rev. Sci. Instrum., 73(10):3409-3439, 2002.

[135] T. K. Popov, P. Ivanova, M. Dimitrova, J. Kovačič, T. Gyergyek, and M. Čerček. Langmuir probe measurements of the electron energy distribution function in magnetized gas discharge plasmas. Plasma Sources Sci. Technol., 21(2):025004, 2012 .

[136] J. Stöckel, J. Adamek, P. Balan, O. Bilyk, J. Brotankova, R. Dejarnac, P. Devynck, I. Duran, J. P. Gunn, M. Hron, et al. Advanced probes for edge plasma diagnostics on the CASTOR tokamak. In Journal of Physics: Conference Series, volume 63, page 012001. IOP Publishing, 2007.

[137] D. Batani, S. Alba, P. Lombardi, and A. Galassi. Use of Langmuir probes in a weakly ionized, steady-state plasma with strong magnetic field. Rev. Sci. Instrum., 68(11):4043-4050, 1997.

[138] K. Dannenmayer, P. Kudrna, M. Tichỳ, and S. Mazouffre. Measurement of plasma parameters in the far-field plume of a Hall effect thruster. Plasma Sources Sci. Technol., 20(6):065012, 2011.

[139] Y. Raitses, D. Staack, A. Smirnov, and N. J. Fisch. Space charge saturated sheath regime and electron temperature saturation in Hall thrusters. Phys. Plasmas, 12(7):073507, 2005.

[140] S. P. Tierno. Diagnostics of classical and dusty plasmas with collecting and emissive Langmuir probes = Diagnosis de plasmas clásicos y granulares mediante sondas colectoras y emisoras de Langmuir. PhD thesis, Escuela Técnica Superior de Ingenieros Aeronáuticos, November 2014. 
[141] O. Troll, L. Conde, E. Criado, J. M. Donoso, and G. Herdrich. Measurements of plasma properties using fast sweep Langmuir probes in unmagnetized weakly ionized plasmas. Contrib. Plasma Phys., 50(9):819-823, 2010.

[142] R. Schrittwieser, P. Balan, M. Hron, C. Ionita, K. Jakubka, L. Kryska, E.o Martines, J. Stöckel, M. Tichy, G. Van Oost, et al. Measurements with an emissive probe in the CASTOR tokamak. Plasma Phys. Control. Fusion, 44(5):567, 2002.

[143] J. P. Sheehan, N. Hershkowitz, I. D Kaganovich, H. Wang, Y. Raitses, E. V Barnat, B. R Weatherford, and D. Sydorenko. Kinetic theory of plasma sheaths surrounding electron-emitting surfaces. Phys. Rev. Lett., 111(7):075002, 2013.

[144] T. Gyergyek and J. Kovačič. Fluid model of the sheath in front of a floating electrode immersed in a magnetized plasma with oblique magnetic field: Some comments on ion source terms and ion temperature effects. Phys. Plasmas, 22(4):043502, 2015.

[145] M. D. Campanell and M. V. Umansky. Strongly emitting surfaces unable to float below plasma potential. Phys. Rev. Lett., 116(8):085003, 2016.

[146] R. Chodura. Plasma-wall transition in an oblique magnetic field. Phys. Fluids, 25(9):1628-1633, 1982.

[147] S. Devaux and G. Manfredi. Vlasov simulations of plasma-wall interactions in a magnetized and weakly collisional plasma. Phys. Plasmas, 13(8):083504, 2006.

[148] R. Chalise and R. Khanal. Self-consistent one dimension in space and three dimension in velocity kinetic trajectory simulation model of magnetized plasmawall transition. Phys. Plasmas, 22(11), 2015.

[149] S. P. Tierno, J. M. Donoso, J. L. Domenech-Garret, and L. Conde. Existence of a virtual cathode close to a strongly electron emissive wall in low density plasmas. Phys. Plasmas, 23(1), 2016.

[150] J. Ou, B. Lin, and X. Zhao. Numerical investigation of plasma-wall interaction during burst of elms in a tokamak device. Phys. Plasmas, 24(1):012510, 2017.

[151] M. A. Lieberman and A. J. Lichtenberg. Principles of plasma discharges and materials processing. Cambridge Univ Press, 1994.

[152] S. P. Tierno, J. L. Domenech-Garret, J. M. Donoso, D. Jennewein, G. Herdrich, S. Fasoulas, and L. Conde. Emissive Langmuir Probes in the Strong Emission Regime for the Determination of the Plasma Properties. IEEE Trans. Plasma Sci., 41(4):695-700, April 2013.

[153] S. Chapman and T. G. Cowling. The mathematical theory of non-uniform gases: an account of the kinetic theory of viscosity, thermal conduction and diffusion in gases. Cambridge university press, 1970. 
[154] O. W. Richardson. Electron emission from metals as a function of temperature. Phys. Rev. D, 23(2):153, 1924.

[155] S. Dushman. Electron emission from metals as a function of temperature. Phys. Rev. D, 21(6):623, 1923.

[156] S. Dushman. Thermionic emission. Reviews of Modern Physics, 2(4):381, 1930.

[157] R. F. Kemp and J. Sellen Jr. Plasma potential measurements by electron emissive probes. Review of Scientific Instruments, 37(4):455-461, 1966.

[158] J. P. Matte and J. Virmont. Electron heat transport down steep temperature gradients. Phys. Rev. Lett., 49:1936-1939, Dec 1982.

[159] M. D. Campanell. Entire plasmas can be restructured when electrons are emitted from the boundaries. Phys. Plasmas, 22(4):040702, 2015.

[160] S. Mazouffre. Electric propulsion for satellites and spacecraft: established technologies and novel approaches. Plasma Sources Science and Technology, 25(3):033002, 2016.

[161] C. Charles. Plasmas for spacecraft propulsion. Journal of Physics D: Applied Physics, 42(16):163001, 2009.

[162] I. D. Boyd. Simulation of Electric Propulsion Thrusters. Technical report, DTIC Document, 2011.

[163] C. Schmidt and A. Piel. Stochastic heating of a single Brownian particle by charge fluctuations in a radio-frequency produced plasma sheath. Phys. Rev. E, 92:043106, Oct 2015 .

[164] K. Ghoos, W. Dekeyser, G. Samaey, et al. Accuracy and convergence of coupled finite-volume/Monte-Carlo codes for plasma edge simulations of nuclear fusion reactors. J. Comput. Phys., 2016.

[165] D. S. Lemons, D. Winske, W. Daughton, and B. Albright. Small-angle Coulomb collision model for particle-in-cell simulations. J. Comput. Phys., 228(5):13911403, 2009.

[166] L. Dagum and R. Menon. OpenMP: an industry standard API for shared-memory programming. IEEE computational science and engineering, 5(1):46-55, 1998.

[167] R. Chandra. Parallel programming in OpenMP. Morgan kaufmann, 2001.

[168] B. Chapman, G. Jost, and R. Van Der Pas. Using OpenMP: portable shared memory parallel programming, volume 10. MIT press, 2008.

[169] G. Hager and G. Wellein. Introduction to high performance computing for scientists and engineers. CRC Press, 2010. 